Florida International University FIU Digital Commons

6-21-2018

\title{
Impact of Person-Environment Fit upon Strain and Well-Being for Emergency Responders
}

April D. Schantz

Florida International University, aschantz@fiu.edu

DOI: $10.25148 /$ etd.FIDC006867

Follow this and additional works at: https://digitalcommons.fiu.edu/etd

Part of the Industrial and Organizational Psychology Commons

\section{Recommended Citation}

Schantz, April D., "Impact of Person-Environment Fit upon Strain and Well-Being for Emergency Responders" (2018). FIU Electronic Theses and Dissertations. 3768.

https://digitalcommons.fiu.edu/etd/3768 


\section{FLORIDA INTERNATIONAL UNIVERSITY}

Miami, Florida

\section{IMPACT OF PERSON-ENVIRONMENT FIT UPON STRAIN AND WELL-BEING FOR EMERGENCY RESPONDERS}

A dissertation submitted in partial fulfillment of the

requirements for the degree of

DOCTOR OF PHILOSOPHY

in

PSYCHOLOGY

by

April D. Schantz

2018 
To: Dean Michael R. Heithaus

College of Arts, Sciences and Education

This dissertation, written by April D. Schantz, and entitled Impact of PersonEnvironment Fit upon Strain and Well-Being for Emergency Responders, having been approved in respect to style and intellectual content, is referred to you for judgment.

We have read this dissertation and recommend that it be approved.

$\begin{array}{r}\hline \text { Paulo A. Graziano } \\ \hline \text { Brooke R. Buckman } \\ \hline \text { Stefany Coxe, Co-Major Professor } \\ \hline \text { Valentina Bruk-Lee, Co-Major Professor }\end{array}$

Date of Defense: June 21, 2018

The dissertation of April D. Schantz is approved.

Dean Michael R. Heithaus

College of Arts, Sciences and Education

Andrés G. Gil

Vice President for Research and Economic Development and Dean of the University Graduate School

Florida International University, 2018 


\section{DEDICATION}

This dissertation is dedicated to the men and women of emergency services.

Across roles and branches of service, these are the amazing people who come to our aid when the situation is most dire; often at the cost of their own health and safety. May the work that is started here continue towards initiatives to facilitate the health and wellbeing of our Emergency Responders. 


\section{ACKNOWLEDGMENTS}

I wish to thank Dr. Valentina Bruk-Lee and Dr. Stefany Coxe as Co-Chairs in my dissertation journey. Their expertise and patience during all phases of this project were essential. My growth as a researcher and writer excelled as a result of their vigilant guidance. Thank you to Dr. Brooke Buckman and Dr. Paulo Graziano who were always available and very supportive as committee members; as well as to our department's Office Assistant, Lara Wilson, for her trustworthy advice in all matters during my time at Florida International University.

I would like to thank Dr. Leslie Frazier, as her mentorship throughout this challenging program was a blessing and represented an example of professionalism I hope to emulate in my own academic career. I wish to thank the Department of Psychology Graduate Student Seed Fund for providing means to send a monetary thankyou to emergency responders who participated in this study.

Lastly, without the feedback, encouragement, and emotional support provided by my family and colleagues, my sanity would have left many months ago. Thank you, to my family who never stopped believing in my abilities; and thank you, to my colleagues in $\mathrm{I} / \mathrm{O}$, I have truly appreciated my time working with and among every one of you. I look forward to hearing of your career and life successes. 


\title{
ABSTRACT OF THE DISSERTATION \\ IMPACT OF PERSON-ENVIRONMENT FIT UPON STRAIN AND WELL-BEING FOR EMERGENCY RESPONDERS
}

\author{
by \\ April D. Schantz \\ Florida International University, 2018 \\ Miami, Florida

\section{Professor Valentina Bruk-Lee, Co-Major Professor \\ Professor Stefany Coxe, Co-Major Professor}

This dissertation used a person-environment fit theoretical framework to examine the influence of person-job misfit as an organizational stressor on strain and well-being outcomes for emergency responders. Independent variables consisted of job attributes such as skill variety, task identity, task significance, autonomy and job-based feedback. These job characteristics are often used in work redesign efforts as they are amendable to organizational change initiatives. Dependent variables included strain outcomes relevant to those working in emergency services: physical symptoms, burnout, and secondary traumatic stress. Also, to include a positive aspect of emergency services work, the wellbeing outcome of compassion satisfaction was examined. Data were collected from 358 emergency responders across the United States via online survey, including law enforcement, firefighters, police/fire/medical dispatch, emergency medical technicians, and paramedics. Methodology utilized polynomial regression analysis in which joint linear and curvilinear effects from two predictors upon one outcome correspond to a three-dimensional response surface reflecting the fit-outcome relationship. This approach 
allowed a detailed examination of the nature of fit and the nature of misfit for each job attribute in relation to strain and well-being. Maximum likelihood with bootstrapping was used to estimate model parameters and test response surface features.

Findings identified several influential fit-outcome relationships including skill variety fitcompassion satisfaction $\left(a_{1}=0.366\right)$, task identity fit-burnout $\left(a_{2}=-0.083\right)$, task significance fit-burnout $\left(\mathrm{a}_{1}=-0.241\right)$ task significance fit-compassion satisfaction $\left(\mathrm{a}_{1}=\right.$ 0.496$,$) , job-based feedback fit-physical symptoms \left(a_{1}=-3.807\right)$, job-based feedback fitburnout $\left(\mathrm{a}_{1}=-0.323\right)$, and job-based feedback fit-compassion satisfaction $\left(\mathrm{a}_{1}=0.391\right)$. In terms of misfit, task identity misfit was related to secondary traumatic stress $\left(a_{3}=-0.209\right)$ and job-based feedback misfit was related to burnout $\left(\mathrm{a}_{3}=-0.234\right)$. Conclusions regarding identified fit-outcome relationships suggested a potential to reduce frequency of physical symptoms, burnout, and secondary traumatic stress and increase employees' experience of compassion satisfaction by considering employees' preference for these job characteristics. On the basis of these findings, opportunities for Emergency Services Management agencies to facilitate wellness for personnel, as well as future research directions are discussed. 


\section{TABLE OF CONTENTS}

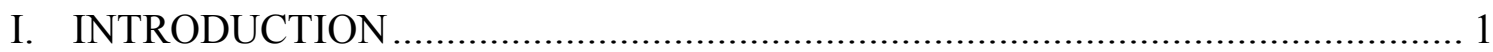

Theoretical Framework: Person-Environment Fit ....................................... 1

Target Industry: Emergency Services ........................................................... 3

Outcomes of Interest: Strain and Well-being ................................................ 4

Necessary Ingredient: Content Dimensions.................................................... 5

JCT: Core Job Dimensions ................................................................ 7

Methodological Approach to the Study of Fit ............................................... 9

Dissertation Organization ................................................................. 10

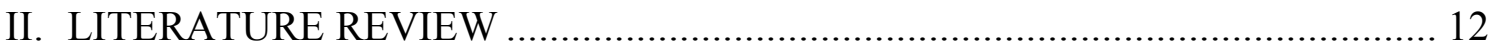

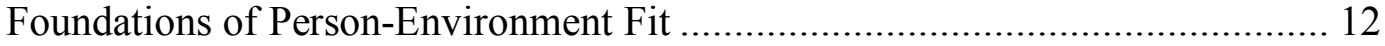

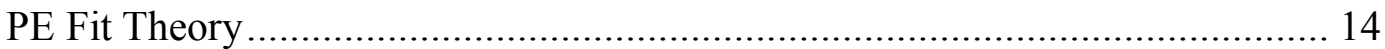

Organization of the PE Fit Research Domain............................................. 17

Forms of PE Misfit Relationships ............................................................. 22

General Relationship between PE Fit and Strain .......................................... 26

Measurement and Analysis Approaches to PE Fit........................................... 27

JCT as Supplemental Theoretical Source for PE Fit Theory............................ 46

Dissertation Outcomes of Interest: Strain and Well-Being ............................. 56

Relationships between Job Characteristics

with Strain and Well-Being Outcomes................................................ 62

Research Questions Addressed in the Dissertation....................................... 74

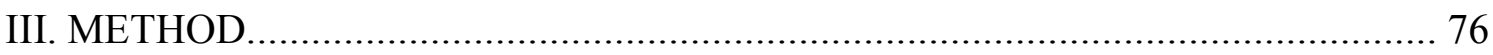

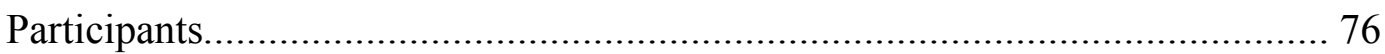

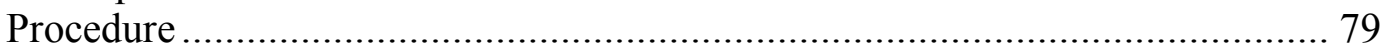

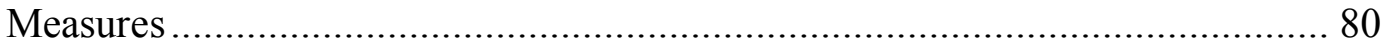

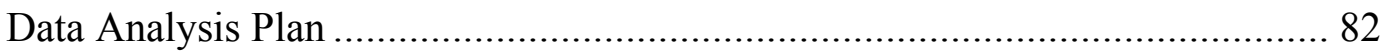

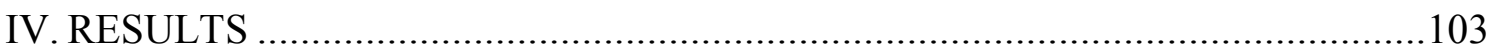

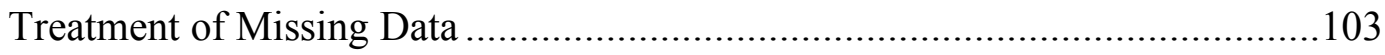

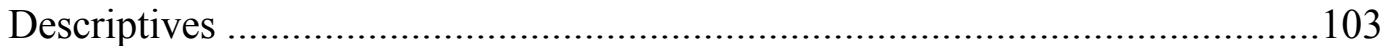

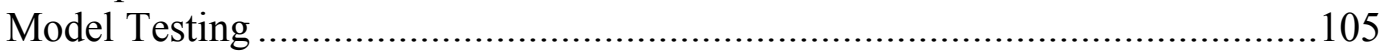

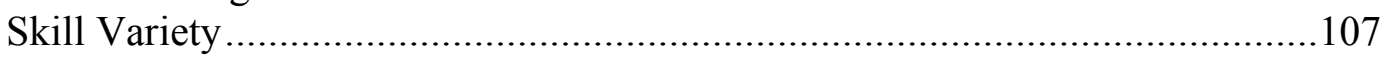

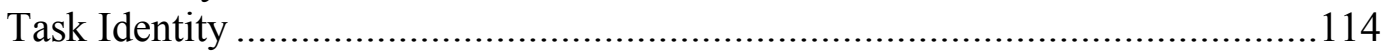

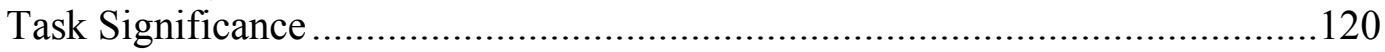

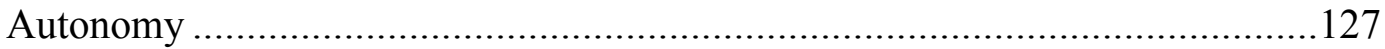

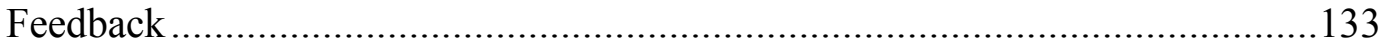

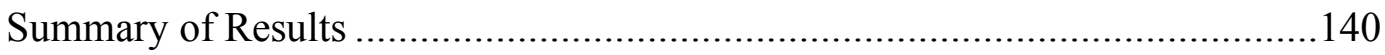




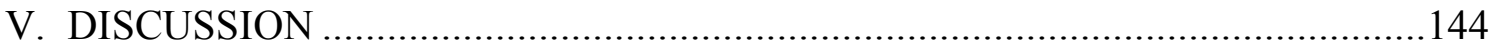

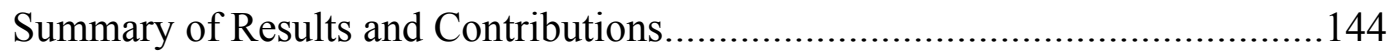

Results and Implications Regarding the Nature of Fit .....................................145

Results and Implications Regarding the Nature of Misfit .................................148

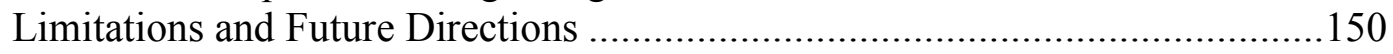

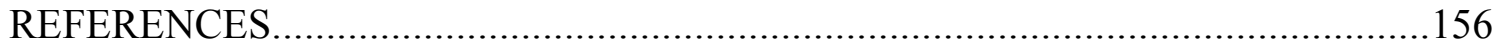

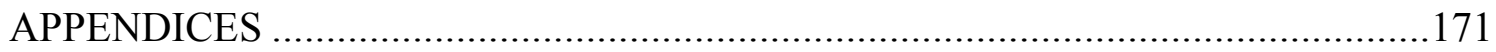

VITA 


\section{LIST OF TABLES}

TABLE

PAGE

A1. Correlations between PE Fit Differences Scores' Transformations and Strains ... 172

A2. Occupational Outlook for Emergency Services Personnel 173

A3. Intercorrelations, Means, and Standard Deviations for Study Variables 174

A4. Regression of Physical Symptoms on Skill Variety Fit 176

A5. Regression of Burnout on Skill Variety Fit 177

A6. Regression of Secondary Traumatic Stress on Skill Variety Fit. 178

A7. Regression of Compassion Satisfaction on Skill Variety Fit. 179

A8. Regression of Physical Symptoms on Task Identity Fit. 180

A9. Regression of Burnout on Task Identity Fit 181

A10. Regression of Secondary Traumatic Stress on Task Identity Fit 182

A11. Regression of Compassion Satisfaction on Task Identity Fit 183

A12. Regression of Physical Symptoms on Task Significance Fit 184

A13. Regression of Burnout on Task Significance Fit 185

A14. Regression of Secondary Traumatic Stress on Task Significance Fit. 186

A15. Regression of Compassion Satisfaction on Task Significance Fit. 187

A16. Regression of Physical Symptoms on Autonomy Fit. 188

A17. Regression of Burnout on Autonomy Fit

A18. Regression of Secondary Traumatic Stress on Autonomy Fit. 190

A19. Regression of Compassion Satisfaction on Autonomy Fit 191

A20. Regression of Physical Symptoms on Feedback Fit

A21. Regression of Burnout on Feedback Fit 
A22. Regression of Secondary Traumatic Stress on Feedback Fit............................. 194

A23. Regression of Compassion Satisfaction on Feedback Fit.................................. 195

A24. Agreement and Discrepancy Percentages for Job Characteristics Dimensions.... 196 


\section{LIST OF FIGURES}

FIGURE

PAGE

1. Job Characteristics Model.................................................................... 7

2. Person-Environment Fit Model........................................................................ 15

3. PE Fit Research Domain....................................................................... 18

4. Three Forms of Excess in Needs-Supplies Fit.................................................23

5. Three Forms of Excess in Demands-Abilities Fit ....................................... 26

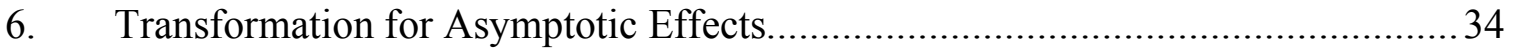

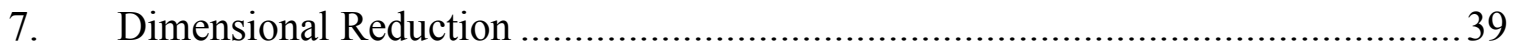

8. Scatterplot of Predictor Space ............................................................... 41

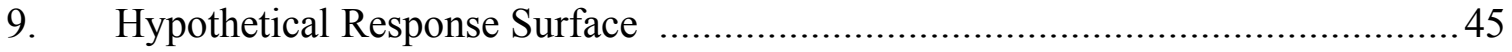

10. Job Characteristics Model..................................................................... 49

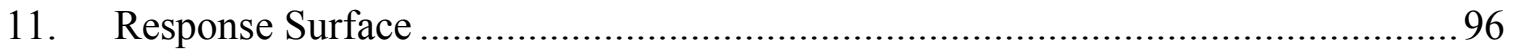

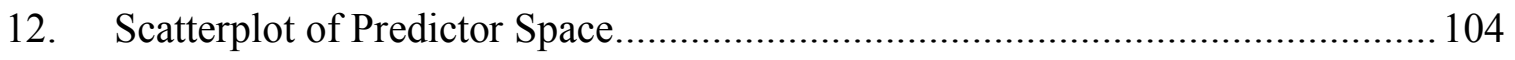

13. Skill Variety Fit with Physical Symptoms .............................................. 109

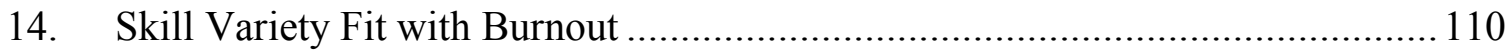

15. Skill Variety Fit with Secondary Traumatic Stress ....................................... 112

16. Skill Variety Fit with Compassion Satisfaction......................................... 113

17. Task Identity Fit with Physical Symptoms ............................................... 115

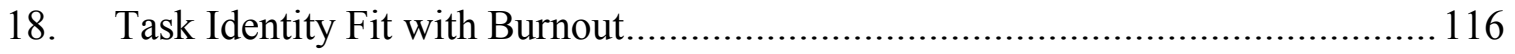

19. Task Identity Fit with Secondary Traumatic Stress .................................... 118

20. Task Identity Fit with Compassion Satisfaction .......................................... 119

21. Task Significance Fit with Physical Symptoms....................................... 121 
22. Task Significance Fit with Burnout

23. Task Significance Fit with Secondary Traumatic Stress

24. Task Significance Fit with Compassion Satisfaction ..................................... 126

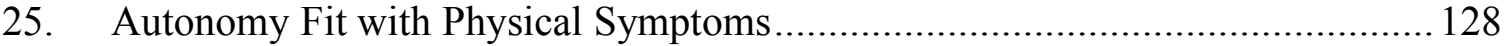

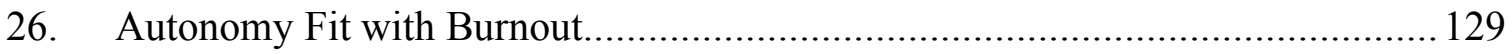

27. Autonomy Fit with Secondary Traumatic Stress ..................................... 131

28. Autonomy Fit with Compassion Satisfaction ......................................... 132

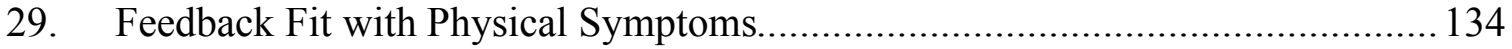

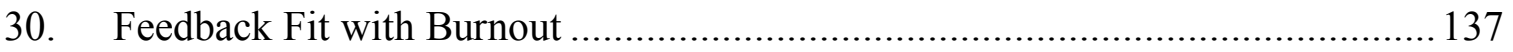

31. Feedback Fit with Secondary Traumatic Stress ...................................... 138

32. Feedback Fit with Compassion Satisfaction............................................ 140 


\section{ABBREVIATIONS AND ACRONYMS}

\begin{tabular}{|c|c|}
\hline ANOVA & Analysis of variance \\
\hline $\mathrm{AuE}$ & Autonomy environment \\
\hline $\mathrm{AuP}$ & Autonomy person \\
\hline $\mathrm{BO}$ & Burnout \\
\hline CS & Compassion satisfaction \\
\hline DFBETAS & Standardized difference in beta coefficients \\
\hline DFFITS & Standardized difference in fit \\
\hline EMT & Emergency Medical Technician \\
\hline FIML & Full information maximum likelihood \\
\hline $\mathrm{FjE}$ & Feedback environment \\
\hline $\mathrm{FjP}$ & Feedback person \\
\hline GNS & Growth-Need-Strength \\
\hline IRB & Institutional Review Board \\
\hline ISR & Institute for Social Research \\
\hline IV & Independent variable \\
\hline JCE & Environment component of job characteristic dimension \\
\hline $\mathrm{JCP}$ & Person component of job characteristic dimension \\
\hline JCT & Job Characteristics Theory \\
\hline JDC & Job Demands Control \\
\hline JDR & Job Demands Resources \\
\hline JDS & Job Diagnostic Survey \\
\hline M & Mean \\
\hline
\end{tabular}




\begin{tabular}{|c|c|}
\hline MAR & Missing at random \\
\hline MCAR & Missing completely at random \\
\hline MI & Multiple Imputation \\
\hline ML & Maximum Likelihood \\
\hline MNAR & Missing not at random \\
\hline MPS & Motivating Potential Score \\
\hline O*NET & Occupational Information Network \\
\hline OLS & Ordinary Least Squares \\
\hline $\mathrm{PE}$ & Person-Environment \\
\hline ProQOL & Professional Quality of Life \\
\hline PS & Physical symptoms \\
\hline PSI & Physical Symptoms Inventory \\
\hline SD & Standard deviation \\
\hline STS & Secondary traumatic stress \\
\hline SvE & Skill variety environment \\
\hline SvP & Skill variety person \\
\hline $\mathrm{TiE}$ & Task identity environment \\
\hline TiP & Task identity person \\
\hline TsE & Task significance environment \\
\hline $\mathrm{TsP}$ & Task significance person \\
\hline URL & Uniform Resource Locator \\
\hline WDQ & Work Diagnostic Questionnaire \\
\hline
\end{tabular}




\section{CHAPTER I: INTRODUCTION}

This dissertation examined the nature of person-environment (PE) fit on strain and well-being outcomes for emergency services personnel. Key terms for the dissertation come primarily from work motivation and organizational stress research literatures. Person-environment fit refers to the match or compatibility between employees and their workplaces (Kristof-Brown \& Guay, 2011). A mismatch between employees' work style preferences and their job's characteristics represents misfit. Within organizational stress research, stressors refer to conditions or events causing subsequent reactions, perceived stress refers to one's perception and appraisal of stressors, and strains refer to psychological, physiological, or behavioral responses to stressors (Bliese, Edwards, \& Sonnentag, 2017). Well-being has been included in quality-of-life research as an indicator of the "global experience of positive reactions to one's life" (Diener, 1994, p. 108) comprised of affective and cognitive components (Hart \& Cooper, 2001). The affective component refers to positive and negative feelings or emotions (Watson, 1988 ) and the cognitive component refers to life satisfaction and satisfaction with various life domains (Pavot \& Diener, 1993). Occupational well-being literature typically focuses on aspects of individual morale or distress, and job satisfaction to tap into affective and cognitive components of well-being in a workplace context (Hart \& Cooper, 2001). In Chapter I, key objectives of the dissertation are highlighted as the theoretical framework, variables of interest, and methodological approach are introduced.

\section{Theoretical Framework: Person-Environment Fit}

Person-environment fit theory was used to guide the design and method of analysis for this dissertation. Consistent with other major theoretical frameworks 
developed from the Institute of Social Research, founders of PE fit theory suggested that both individual differences and workplace characteristics should be used concurrently to study workplace stress (Beehr \& Newman, 1978; Caplan, Cobb, French, Harrison, \& Pinneau, 1980; French, Caplan, \& Harrison, 1982; French \& Kahn, 1962; Harrison, 1976, 1978). As such, the PE fit approach included individual and work-related input variables not typically used by other theories of that timeframe.

In the framework of PE fit theory, a stressor is further defined as "a subjective appraisal indicating that supplies are insufficient to fulfill the person's needs, with the provision that insufficient supplies may occur as a consequence of unmet demands" (Edwards, Caplan \& Harrison, 1998, p. 32). The PE fit theory defines a strain as a deviation from a normal state, as seen in individuals' psychological, physical, or behavioral responses (French et al., 1982). Organizational stressors, such as misfit between a person and their job, have been found relevant to employee outcomes such as strain, satisfaction, and burnout (Caplan et al., 1980; French et al., 1982; Harrison, 1978; Kristof-Brown, Zimmerman, \& Johnson, 2005). Therefore, the first key objective of the dissertation was to identify job characteristics in which fit, or misfit, was related to strain or well-being.

While various types and forms of fit are examined in the workplace environment, the dissertation focused on person-job fit as indicated by one's preference for and the perceived presence of specific job characteristics. Type of fit, along with foundations, development, and methodological approaches to the study of fit, are discussed in detail in Chapter II. 


\section{Target Industry: Emergency Services}

Emergency responders represent a work-role in which the outcomes associated with workplace stressors have high-stakes consequences. Reported disadvantages to emergency services work include the likelihood of working in dangerous environments, irregular or long work hours, and the physical and emotional stress inherent in these positions (U.S. Bureau of Labor Statistics, 2015). Research has confirmed several stressor-strain relationships for emergency response personnel, usually attributed to a lack of support or control in an increasingly complex and demanding job, coupled with frequent exposure to trauma (Duxbury \& Higgins, 2012; Grigsby \& McKnew, 1988; Regehr, 2009; Regehr, Goldberg, \& Hughes, 2002). Organizational characteristics and job demands have been found related to strain outcomes (e.g., burnout, generalized strain, post-traumatic stress) for paramedics (Grigsby \& McKnew, 1988; Regehr et al., 2002), nurses (Huyghebaert, Gillet, Lahiani, \& Fouquereau, 2016), police (Martinussen, Richardsen \& Burke, 2007), corrections officers (Griffin, Hogan, \& Lambert, 2012), and firefighters (Regehr, 2009) to name a few. The dissertation addresses the relationship between misfit as an organizational stressor and employee strain and well-being outcomes for emergency responders.

While there are several aspects of emergency services work that contribute to strain, the dissertation focused on job characteristics relevant to emergency services personnel that might be addressed by organizational change initiatives. Specifically, while some job characteristics are rarely controllable (e.g., being shot at while working as a police officer), other job characteristics have the potential to be addressed by the organization (e.g., variety of skills used, decision latitude). Identifying which 
organizational stressors are relevant to strain outcomes for emergency services personnel represents the second key objective of the dissertation. An occupational profile is provided in Chapter III to better understand the occupational outlook and industry characteristics for emergency services personnel.

\section{Outcomes of Interest: Strain and Well-being}

Outcomes chosen for the dissertation were determined by their relevance to emergency responders, which included physical symptoms, burnout, secondary traumatic stress, and compassion satisfaction.

Physical symptoms (also called somatization or somatic symptoms) describe the presence of medically unexplained symptoms. They can include experiences such as headaches, digestive disturbances, or eye/ear strain. Typically an unconscious process by which psychological distress is expressed, persistent somatization is associated with increased rates of disability and health care utilization (Schat, Kelloway, \& Desmarais, 2005).

Negative aspects of providing care to those who have experienced trauma has been defined by the term compassion fatigue and is comprised by two sub facets: burnout and secondary traumatic stress (Stamm, 2010). The first sub facet, burnout, refers to feelings of frustration, exhaustion, anger, and depression. The second sub facet, secondary traumatic stress, is characterized by a mental preoccupation of people one has helped and is driven by fear and work-related trauma exposure (Figley, 1999).

Compassion satisfaction represents a well-being outcome related to emergency services work. Compassion satisfaction reflects one's pleasure in being able to effectively handle the challenges of emergency services, as well as in helping others through one's 
work. It is typically associated with positivity towards one's colleagues and workgroup, and with one's feelings of personal ability to contribute to the work setting or towards the greater good of society (Stamm, 2010). Compassion satisfaction, as a positive aspect of emergency services work, was included in the dissertation to provide a balanced assessment of the impact of PE fit for emergency responders.

While overall associations between PE fit and strain outcomes have been identified, limited research has been conducted regarding fit in these specific strain outcome areas. As examples, Edwards and Harrison (1993) found job complexity fit related to job dissatisfaction and boredom and Chilton, Hardgrave, and Armstrong (2005) found cognitive style fit related to tension and dissatisfaction. However, fit related to strain outcomes such as compassion satisfaction and secondary traumatic stress have not been examined. Overall, in a meta-analysis conducted by Kristof-Brown and colleagues (2005), a moderate correlation of $\hat{\rho}=-.28(k=10, N=3,505)$ was found between PE fit and strain in general. As specific types of strain outcomes were not categorized, many opportunities exist to examine details of these stressor-strain relationships through the lens of PE fit.

\section{Necessary Ingredient: Content Dimensions}

While PE fit theory describes the mechanism of fit, a context specific framework is required to guide hypotheses development according to areas of interest. Specifically, PE fit theory requires study variables and their expected associations to be supplied from another theoretical source. Job Characteristics Theory (JCT) by Hackman and Oldham $(1976 ; 1980)$ was chosen as the theoretical framework for job characteristics predictor

variables. Specifically, the Job Characteristics Model (Hackman \& Oldham, 1976) was 
developed to explain how job design affects work motivation. The primary objective of JCT was to have an actual impact on job design through the assessment and identification of areas that needed redesign and to evaluate effectiveness of redesign efforts (Hackman \& Oldham, 1976, 1980). This dissertation shares that objective to promote job redesign efforts with evidence-based guidance. It is hoped that identification of job fit dimensions amendable to change would provide support and guidance for these types of organizational initiatives. Job characteristics utilized as content dimensions included skill variety, task identity, task significance, autonomy, and feedback.

The basic premise of JCT is that employees experience work motivation to the extent one learns that they have performed well on a task that is meaningful to them (Hackman \& Oldham, 1976, 1980). Specifically, Hackman and Oldham $(1976,1980)$ posited that these five core job characteristics dimensions influence one's perception of meaningfulness, experienced responsibility, and knowledge of results, which then impact personal and work outcomes (see Figure 1). The dissertation uses these core job dimensions as person and environment components. 


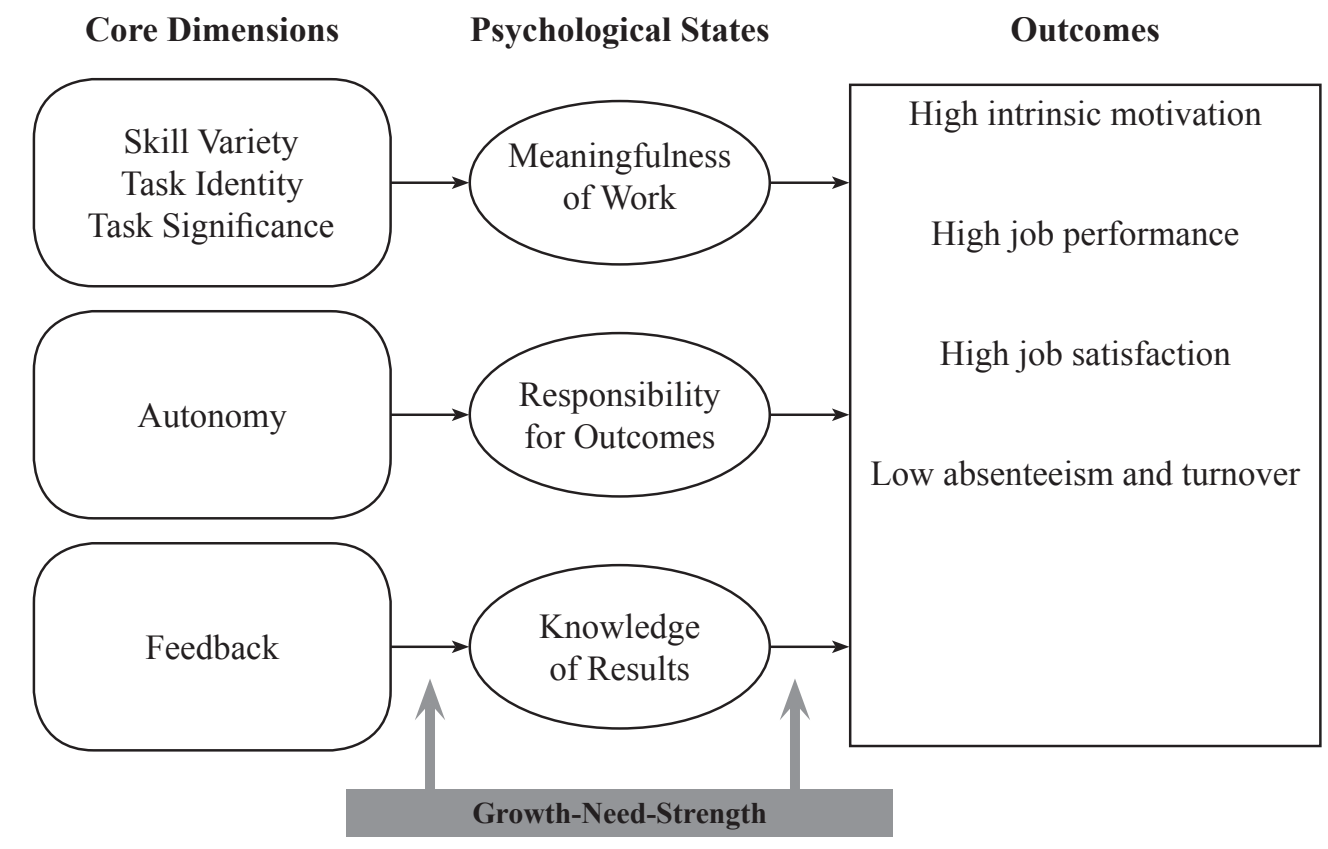

Figure 1. Job Characteristics Model.

\section{JCT: Core Job Dimensions}

Skill variety refers to work activities that challenge employee's abilities, skills, and talents. When a task requires an employee to engage in activities that challenge or stretch their abilities, they tend to experience that activity as meaningful. Similarly, the more skills involved in a task, the more meaningful that task may be perceived to be. However, for individuals who prefer straightforward, one-goal type tasks, a high level of skill variety may be perceived as stressful and an overload to their work responsibilities.

Task identity refers to the degree in which a job requires completion of a whole and identifiable piece of work. Individuals who prefer a high level of task identity desire work that allows them to see a task from start to finish; and may derive more meaningfulness of their work when they are able to stay involved in a work process through its stages. How one's preference for task identity coupled with the job's supply 
of opportunities to see a crisis response event from start to finish is representative of the concept of PE fit focused upon in the dissertation.

Task significance refers to the extent one's task impacts the lives others - whether within or external to the organization. Task significance contributes towards meaningfulness, as results of emergency services work have a rather obvious impact on the well-being of others. An emergency responder could also perceive tasks such as maintenance of equipment or maintaining personal readiness as a significant part of impacting others' lives.

Autonomy refers to the degree to which a job allows freedom and independence in how the work is executed. Latitude in establishing priority and execution of tasks prompts employees towards feelings of personal responsibility for their work outcomes. As jobs with high autonomy are dependent on individual's own efforts, initiatives, or decisions, emergency responders might or might not prefer a high level of autonomy when others' lives are at stake.

Feedback from the job refers to the degree to which carrying out work activities required by the job provides an employee with direct and clear information about their performance effectiveness (Hackman \& Oldham, 1976, 1980). While feedback from agents (such as from coworkers or supervisors) also provides information about one's performance, feedback that results from the job itself provides proximal, objective information in a timelier manner. Both positive and negative feedback are considered beneficial to learning (Elder, Barkhuizen, Knoch, \& von Randow, 2007) and have been found critical to employee growth and performance (Glover, 2000; Hackman \& Oldham, 1976, 1980; Helgoe, 2010). However, an individual's readiness or preference for 
feedback may affect his/her reception of feedback. In other words, the focus of the dissertation is not limited to an individual's preference for feedback, but considers the match or congruence between an individual's preference for feedback and the supply of feedback opportunities from the job.

In summary, core job characteristics from Hackman and Oldham's $(1976,1980)$ JCT were modified to provide person and environment variables to examine person-job fit. A more detailed discussion of these job characteristics dimensions in the context of PE fit theory is provided in Chapter II: Literature Review.

\section{Methodological Approach to the Study of Fit}

The dissertation examined JCT core job dimensions expected to maximize wellbeing outcomes (i.e., compassion satisfaction) or reduce strain outcomes (i.e., physical symptoms, burnout, and secondary traumatic stress). Previous research examining fitoutcome relationships has found effects of congruence between preferred and supplied job attributes best modeled by a polynomial regression approach (Edwards, 2002; Kristof-Brown et al., 2005). The polynomial equation, which models a fit-outcome relationship with two predictors with an outcome, corresponds to a three-dimensional response surface (Box \& Draper, 1987). The surface visually represents the relationship between multiple predictors (i.e., person and job characteristics) and the strain or wellbeing outcome. Examining fit-outcome relationships using polynomial regression and response surface analysis provides a more nuanced view of fit-outcome relationships than the previously used methodology of difference scores (Edwards, 2002; Shanock, Baran, Gentry, Pattison, \& Heggestad, 2010). Using polynomial regression, both person and 
environment components are retained and taken into account to answer questions about fit-outcome relationships.

Questions of interest include:

- What is the relationship with strain and well-being outcomes when one's preference for and perceived presence of a job attribute are matched?

- When there is a discrepancy between one's preference for and presence of a job attribute, does this discrepancy relate to strain and well-being outcomes?

In summary, polynomial regression with response surface analysis was selected to test the nature of fit and the nature of misfit (Edwards \& Shipp, 2007; Shanock et al., 2010). Use of this analysis technique represents the final key objective of the dissertation. A thorough discussion of approach methods for fit research is provided in Chapter II, with an analysis plan that discusses how response surfaces are derived and tested in Chapter III.

\section{Dissertation Organization}

Chapter II provides the foundation and development of the PE fit research domain, including previously used methodological approaches and the current approach of polynomial regression with response surface analysis. Chapter II also discusses the theoretical basis of the selected job characteristics predictors and organizational stress outcomes of interest, as well as previously found relationships between these variables. Research questions posed in the dissertation conclude Chapter II.

Chapter III presents details of the target population of emergency responders and describes measurements used in the online survey. The Data Analysis Plan in Chapter III 
provides details regarding the use of polynomial regression and response surface analysis to describe resulting fit-outcome relationships. Results obtained to answer research questions are provided in Chapter IV, with detailed exploration of the response surface for each fit-outcome relationship. Chapter V summarizes results and contributions of the dissertation regarding the nature of fit and the nature of misfit. Limitations and future directions conclude Chapter V and the dissertation. Figures are embedded within the dissertation to provide convenient access to response surface representations, while tables and other supplementary material are provided in Appendices. 


\section{CHAPTER II: LITERATURE REVIEW}

This literature review presents theoretical frameworks used to organize the dissertation. Namely, how job motivation and organizational stress are tied together by the focal framework of person-environment fit. Chapter II begins with the development of person-environment fit theory as a mechanism of job stress (French et al., 1982). A review of the current research domain of person-environment fit, previous and current approaches used to operationalize fit, and details regarding the dissertation's approach,

polynomial regression and response surface analysis, are presented. A discussion of work motivation as explained by the Job Characteristics Theory follows, which provides predictor dimensions of interest. Chapter II concludes with a review of previous research focused on relationships between job characteristics dimensions and strain and well-being outcomes of interest, especially highlighting research relevant to the context of emergency services.

\section{Foundations of Person-Environment Fit}

As previously introduced, French and colleagues (Caplan et al., 1980; French et al., 1982; Harrison, 1978) developed the concept of person-environment (PE) fit in association with a then-nascent program at the Institute for Social Research (ISR) at the University of Michigan (French \& Kahn, 1962). The ISR program focused on effects of environmental factors on mental health. Specifically, they sought to examine social psychological factors in large scale organizations, contribute to an integrated theory of personality and organization, and offer an improved understanding of the effects of these factors on health and illness. Founders of the ISR program viewed the organizational environment not only as a source of pressures and conflicts that interfered with mental 
health, but also recognized the environment as a source of support conducive to mental health (French \& Kahn, 1962).

As a derivative of the original ISR framework, PE fit theory of job stress distinguishes objective person and environment factors from their subjective counterparts and highlights misfit between the subjective person and environment as a key determinant of psychological, physiological, and behavioral strains (Bliese et al., 2017; French et al., 1982). As previously introduced, PE fit theory defines job stress as the misfit between an employee and their job environment, which in turn threatens individuals' well-being and results in strain outcomes (Beehr \& Newman, 1978; Caplan et al., 1980; French et al., 1982; Harrison, 1978). While PE fit theory was originally developed to test hypotheses regarding the goodness-of-fit between individuals and their jobs on risk factors for coronary heart disease (French et al., 1982), it has also been used to examine fit-outcome relationships for other criteria, such as performance, satisfaction, and turnover intent (see Kristof-Brown et al., 2005).

The concept of PE fit is ubiquitous, not only in stress research, but also in many areas of organizational behavior, including job satisfaction (Locke, 1976), work adjustment (Dawis \& Lofquist, 1984), personnel selection (Saks \& Ashforth, 1997; Schneider, 1987), and work redesign (Hackman \& Oldham, 1976, 1980). Foundational theoretical work foreshadowing PE fit theory has included Parsons' matching model for career decision making, Murray's need-press model, and Lewin's field theory (as cited in Edwards, 2008). On the basis of interactionist psychology, these perspectives stated that person and environment characteristics interact to affect behavior (Kulka, 1979; Terborg, 1981). Discrepancy or comparison-based theories have also contributed to the current 
understanding of PE fit through comparisons between work environment and personal need factors (e.g. Cummings \& Cooper, 1979; Locke, 1976; Porter, 1962).

\section{PE Fit Theory}

Current conceptualization of PE fit refers to the match, congruence, or similarity between the person and environment (Edwards et al., 1998; Muchinsky \& Monahan, 1987; Schneider, Kristof, Goldstein, \& Smith, 1997). Person-environment fit theory in its own right began with studies conducted at ISR that aimed to describe occupational differences in stress and strain among diverse occupations (Caplan et al., 1980; French et al., 1982). French and colleagues suggested that an understanding of how to reduce psychosocial stressors was just as relevant to workplace initiatives as the reduction of physically hazardous work conditions. Specifically, ensuring a physically safe environment was important, but only minimally rewarding if employees must constantly struggle with boredom or the stress of being pushed beyond their motivational and intellectual limits (Caplan et al., 1980; French et al., 1982). Figure 2 presents the original framework of PE fit theory as developed by French and colleagues (Caplan et al., 1980; French et al., 1982; Harrison, 1978), which is followed by a brief description of this foundational framework. 


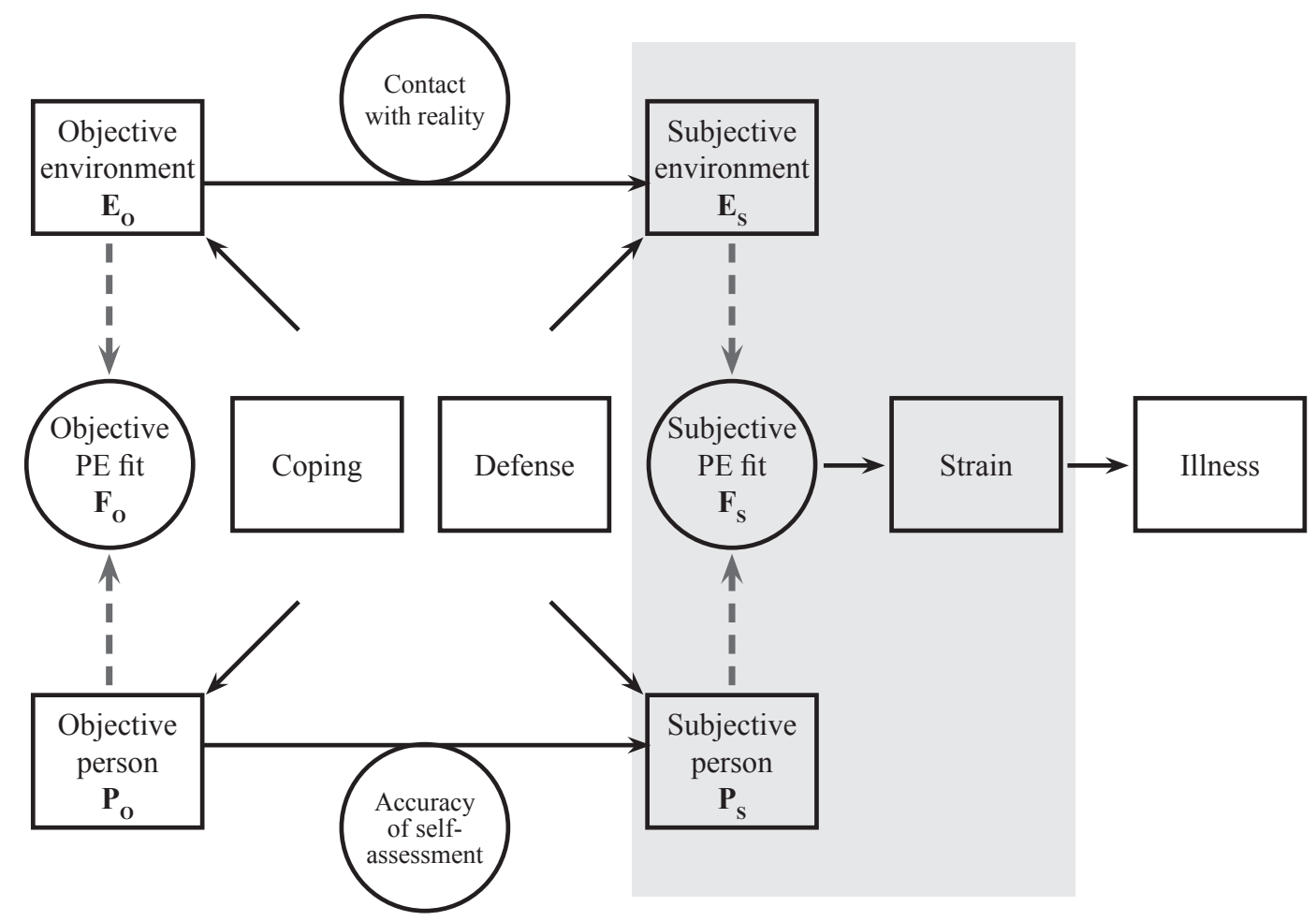

Figure 2. Person-Environment Fit Model of psychosocial stress in terms of fit between the person $(\mathrm{P})$ and the environment $(\mathrm{E})$. Concepts within circles are discrepancies between the two adjoining concepts. Solid lines indicate main effects; dashed lines indicate potential joint additive effects of $\mathrm{P}$ and $\mathrm{E}$. Gray area indicates portion of model focused upon in the dissertation.

First, note the distinction between person and environment, which is essential for the conceptualization of PE fit and represents the reciprocal causation of the person and environment in fit effects (Edwards et al., 1998). The second item to note is the distinction between objective and subjective representations of the person and environment. Objective person refers to the person's characteristics and attributes as they truly exist, whereas objective environment refers to the physical and social environment independent of the person's perception of it. Objective PE fit refers to the fit between the person and the environment independent of the individual's perceptions of it (Harrison, 1978). 
The subjective person refers to one's perceptions of their own needs, values, abilities, or other attributes. Subjective environment refers to a person's perceptions of the objective environment. Specifically, one's psychological construction of the world in which they live and work, which includes perceptions of supplies available to meet personally relevant needs and perceptions of demands required to perform and be successful at their jobs (Harrison, 1978).

Two concepts in circles, contact with reality and accuracy of self-assessment, represent inaccuracies in the person's subjective perception of their objective world and of themselves respectively. While subjective perceptions may be more or less accurate representations of their objective counterparts, the model assumes that a person's responses are directly determined by these subjective variables. Therefore, subjective PE fit refers to the interaction between the subjective person and the subjective environment, which has been identified as a key predictor of strain outcomes (French et al., 1982).

Coping and defense represent a person's efforts to improve objective PE fit or enhance their subjective PE fit respectively (Edwards et al., 1998). Specifically, coping refers to responses that actively address poor fit by changing the attributes of the objective person or taking action to master the objective environment. Defense refers to changing one's perception of fit (e.g., cognitive reframing, denial) without actually addressing objective $\mathrm{P}$ and $\mathrm{E}$ counterparts.

As introduced in Chapter I, PE fit theory defines a stressor as "a subjective appraisal indicating that supplies are insufficient to fulfill the person's needs, with the provision that insufficient supplies may occur as a consequence of unmet demands" (Edwards et al., 1998, p. 32). Person-environment fit defines a strain as a deviation from 
normal state, as seen in individuals' psychological, physical, or behavioral responses (French et al., 1982). Psychological strains have included work attitudes and affective strains such as anger, anxiety, depression, and psychosomatic or post-traumatic stress symptoms. Physiological strains include increased cortisol levels or high blood pressure and examples of behavioral symptoms of strain include over-eating, increased frequency of smoking, and absenteeism (Griffin \& Clarke, 2011; Harrison, 1978).

Essentially, good fit results when a match between person and environment is present, such as when a job environment provides supplies desired by the person, while the person provides abilities required by the job environment (Harrison, 1978). In the context of PE fit theory, stress is caused by an environment that does not provide adequate supplies to meet the person's needs or by a person's insufficient abilities to meet demands that are prerequisite to receiving supplies. Therefore, the degree of misfit between person and environment represents an organizational stressor, which leads to strain and potentially to long-term illness as shown in Figure 2 (Edwards et al., 1998; French et al., 1982; Harrison, 1978). Of note, the dissertation focused on subjective fit as indicated by joint effects from subjective environment and subjective person on strain outcomes, as highlighted in the gray box in Figure 2.

\section{Organization of the PE Fit Research Domain}

To date, a considerable amount of theoretical development and research has investigated the relationship between PE fit and outcomes (see Kristof-Brown et al., 2005). However, this has caused a proliferation of conceptualizations, measures, and analytic approaches which make this construct elusive and unwieldy (Judge \& Ferris, 1992; Kristof-Brown et al., 2005). Edwards and Shipp (2007) presented a general 
integrative framework for theorizing the effects of PE fit, which can be applied and extended in various specific streams of PE fit research. Edwards and Shipp's (2007) extended framework was used to guide further discussion of PE fit theory and to situate the dissertation within the current research domain. As shown in Figure 3, the integrative framework defines the PE fit domain space by type of fit, level of the environment, and content dimensions; all of which have been used as primary approaches in PE fit research (Edwards \& Shipp, 2007).

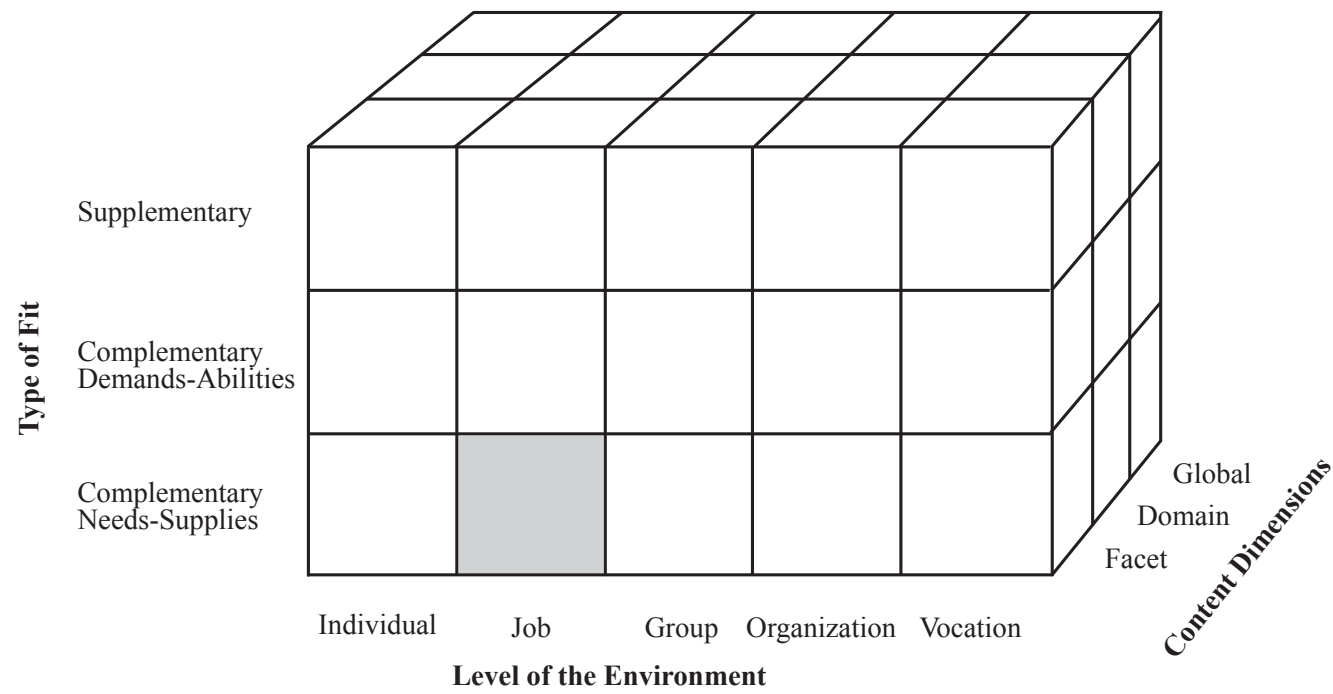

Figure 3. PE Fit Research Domain illustrates integrative framework, which includes various approaches used in specific streams of PE fit research. Gray area represents the focus of this dissertation situated within the integrative framework.

\section{Types of PE fit.}

Supplementary fit derives from similarity, such that a person fits into some environmental context because he or she possesses characteristics that are similar to other individuals in the environment, where the environment refers to other people individually, or collectively in groups, organizations, or vocations (Muchinsky \& 
Monahan, 1987). Supplementary fit theories emphasize compatibility on the basis of similarity between people and some aspect of their environment. Supplementary fit has roots in the attraction-selection-attrition framework, which posits that individuals are attracted to and stay in organizations that are compatible with their personalities (Schneider, 1987). Supplementary fit is often used to examine macro-level content dimensions in which the similarity between a person and vocation/organization would facilitate a smoother working environment (see Kristof-Brown et al., 2005).

Complementary fit theories emphasize compatibility defined by completion, such that "characteristics of an individual serve to 'make whole' or complement the characteristics of an environment" (Muchinsky \& Monahan, 1987, p. 271). Complementary fit is further conceptualized into two sub-types. The degree to which environmental demands are fulfilled by an individual's knowledge, skills, abilities, and other characteristics (KSAOs) refers to demands-abilities fit (French et al., 1982). Whereas, from the person's perspective, needs-supplies fit refers to a work environment that fulfills a person's needs, desires, motives and/or goals (Caplan, 1987; French et al., 1982). Complementary fit type has been utilized at a job or individual level. Regardless of whether the environment is facilitating the "achievement of one's job values" (Locke, 1969, p. 316) as in needs-supplies fit, or the individual's KSAOs are sufficient to meet the environmental job demands as in demands-abilities fit, a complementary fit type identifies this symbiotic relationship.

\section{Levels of the environment.}

While much research examines PE fit as similarities between the person and the organization, explicit designation of fit type apart from environmental level clarifies the 
purpose and position of each study within the theoretical domain of PE fit. The level of environment in PE fit research often obscures distinctions between supplementary, demands-abilities, and needs-supplies fit types. Respondents may be asked about how well they fit to a job, organization, or vocation without specifying whether this perception of fit should be based as a supplementary or complementary type of fit. Within supplementary fit a person's similarity can be compared to other individuals (e.g. supervisors, coworkers), or collectively compared to members of the group, organization, or vocation. From a demands-abilities approach, demands can be exclusive to the experiences of an individual, shared by all incumbents of a specific job, or members of a work group, department, organization, or vocation (Edwards \& Shipp, 2007). Likewise, when examining needs-supplies fit, supplies provided to meet individual needs could come from resources made available to the workgroup, department, organization, or vocation. In other words, while PE fit research conceptualizes the person at individual level, the environment can be framed at different levels. Using an integrated model helps to specify all aspects of inquiry and prevents misunderstandings between research designs to enable accurate comparisons between studies.

\section{Content of person and environment dimensions.}

The PE fit domain is also defined by the level of specificity in content dimensions of interest, which can range on a continuum from general to specific. Points along the specificity continuum used to demarcate content dimensionality are termed global, domain, and facet levels of specificity (Edwards \& Shipp, 2007).

Global represents the general extreme of the specificity continuum as it refers to content in a broad sense, without reference to any dimensions of comparison. Examples 
of global level would be in asking respondents about their overall similarity, overall fit between demands and abilities, or assessing their general perceptions of needs fulfillment (Edwards, Cable, Williamson, Lambert, \& Shipp, 2006).

Domain isolates broad areas of comparison but does not distinguish dimensions within each area. Examples of this level would be found in comparisons of values, goals, personality, or demographic characteristics without going into specifics within these areas. Domain level concerns fit on general need and supply dimensions, or broad distinctions among demand and ability dimensions (Edwards et al., 2006).

Facet is used to label the specific extreme of this content dimension continuum and examines dimensions within broader areas (Edwards et al., 2006). Examples of facet level specificity can be seen through inquiries about specific tasks or activities (e.g., creative or innovative problem solving, intra/inter-group communication) or regarding job scope separated into facets (e.g., autonomy, skill variety, task identity). Accordingly, fit at the facet level of specificity for job characteristics dimensions is examined in the dissertation, as indicated by the gray section in Figure 3. Of note, while PE Fit theory describes the mechanism by which person and environment jointly influence strain, it does not specify what content should be used for person and environment dimensions (Edwards et al., 1998).

Central to PE fit theory in respect to the content of person and environment dimensions is that the measurement of dimensions must be commensurate (Dawis \& Lofquist, 1984; Edwards \& Shipp, 2007: French et al., 1982). Commensurate measures express the person and the job in terms of the same content dimensions (Caplan, 1987; Edwards, 1991). For example, commensurate measures of fit between employee desires 
and supplies of benefits would assess the benefits desired by the employee and, in a similar fashion, the benefits package offered by the organization. Combining a measure of benefits offered by the organization with desires regarding any job attribute other than desired benefits would render the comparison meaningless in terms of PE fit (Edwards, 1991). Commensurate measures ensure conceptual relevance of person and environment measures to one another and are essential to enable interpretation of PE misfit (Caplan, 1987).

\section{Forms of PE Misfit Relationships}

The PE fit theory has specified three potential relationships between misfit and strain as shown in Figure 4. In this illustration, the horizontal axis represents the degree of discrepancy between person and environment (i.e., needs and supplies). Negative scores indicate a deficiency in that supplies fell short of needs and positive scores represent excess in that supplies exceeded needs. Zero at the center of the scale represents the point of perfect fit where environment and person scores were equal. The vertical axis represents a strain outcome (e.g., burnout). 


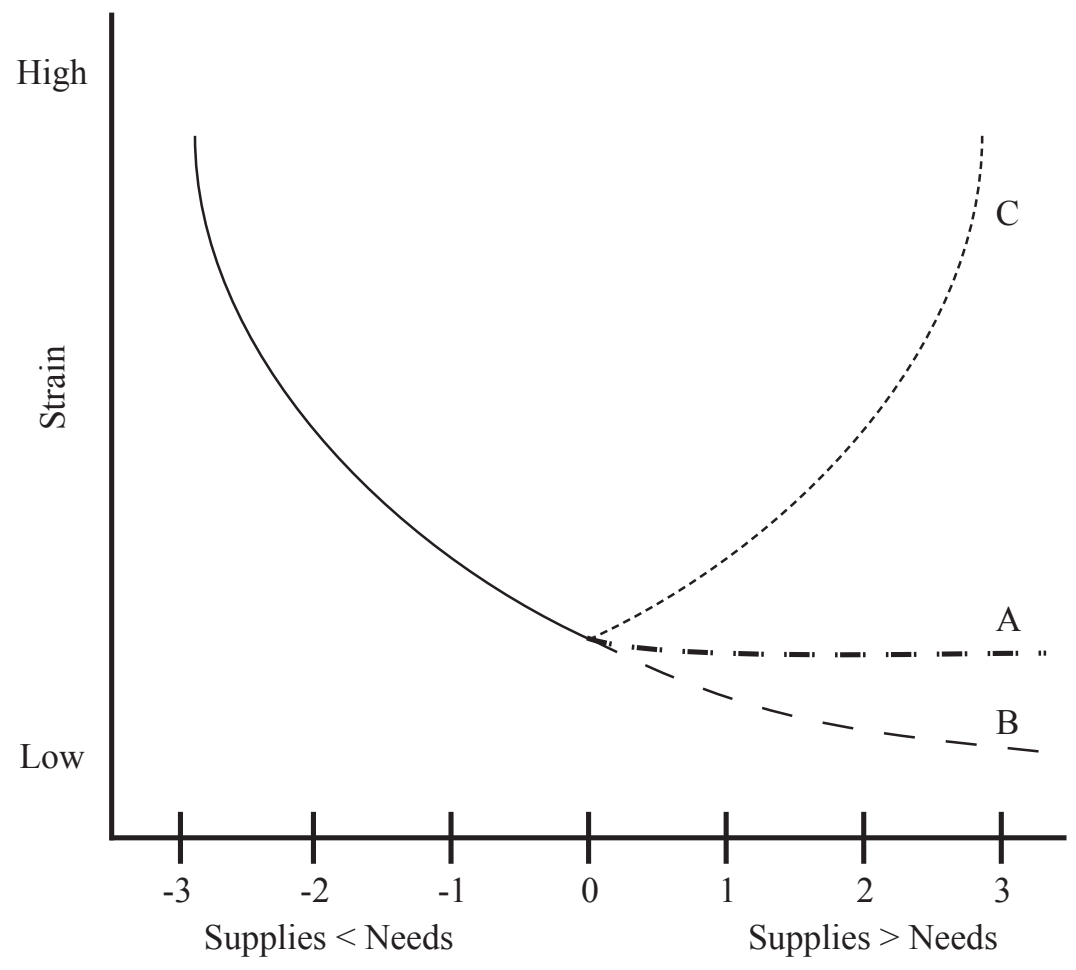

Figure 4. Three Forms of Excess in Needs-Supplies Fit represents three potential effects resulting from excess in supplies on strain: no further effect associated with excessive supplies (asymptotic, Curve A), continued reduction in strain associated with the excess in supplies (monotonic, Curve B), and increased strain associated with excess in supplies (parabolic, Curve C).

On the left side of Figure 4, the solid line shows that as supplies increase to meet needs, strain is reduced. This effect is consistent for all needs-supplies dimensions. For example, insufficient supply relative to one's needs for a characteristic (e.g., belonging, compensation, companionship) result in increased strain; whereas supply that meets one's needs result in decreased strain. However, when supplies surpass needs, three potential effects may result as indicated by the three dashed curves on the right side of the illustration (Harrison, 1978). First, curve A indicates no further effect on strain resulting from excess in supplies. For example, food and water will reduce strain until hunger and 
thirst are met. Additional supply of food and water after satiety do not continue to reduce strain, defined as an asymptotic relationship between needs-supplies fit and strain. Second, curve B illustrates a monotonic relationship between needs-supplies and strain in which excess in supplies in one dimension can be used in the future, either for that same dimension or used to relieve needs in a different dimension. These mechanisms are defined as conservation and carryover, respectively (Edwards, 1996). An example of conservation would be when excess in funds beyond one's budgetary needs can be saved for upcoming expenses; whereas an example for carryover would be when excess in decision latitude in one's specific job permits one to address other areas at work. Finally, curve $\mathrm{C}$ illustrates a parabolic or U-shaped relationship, such that excess supplies cause increase in strain outcomes. A parabolic relationship can occur when excess in supplies inhibits the fulfillment of needs on other dimensions or deplete the availability of supplies that would fulfill needs in the future. These are defined as interference and depletion, respectively (Edwards, 1996; Harrison, 1978). Interference or depletion that result from excess is indicated by a parabolic or curvilinear functional form in which strain outcomes increase as supplies exceed individual's needs (Edwards, 1996; Edwards et al., 1998; Harrison, 1978). For example, if one's need for task significance is oversupplied, this may interfere with their need for separation between work and home domains. Similarly, excess in resources obtained from one's supervisor for one event may deplete the provision of resources for a future event.

Relationships between demands-abilities fit type and strain are similar to those between needs-supplies fit type and strain (Figure 5). In the demands-abilities illustration, the solid line on the right indicates a monotonic strain increase as demands exceed one's 
abilities, which is an effect universal across content dimensions. In other words, excessive job-related demands (e.g., required use of multiple skills) result in increased strain; whereas increases in abilities to meet demands decrease strain. When one's abilities exceed demands needed by the job as illustrated on the left side of Figure 5, a set of similar potential forms of effect are shown by the three dashed curves (Harrison, 1978). Curve A indicates an excess in abilities that has no relationship with strain and results in an asymptotic effect. For example, an asymptotic effect may result when excess in knowledge (e.g., mathematics) provides no benefit to other demand or need areas. Curve B indicates a relationship in which excess in abilities provides a means to accomplish other needs or meet other demands (i.e., conservation, carryover). An example would be in one's abilities to easily handle the workload, providing opportunities for family time, exercise, or other activities. And finally, curve $\mathrm{C}$ shows an excess in abilities related to increased strain. An example of this relationship would be when one's abilities are not fully used or appreciated, and the underutilization of one's skills interferes with other unmet needs for usefulness (Caplan, 1987; French et al., 1982; O’Brien, 1983; Schneider et al., 1997). 


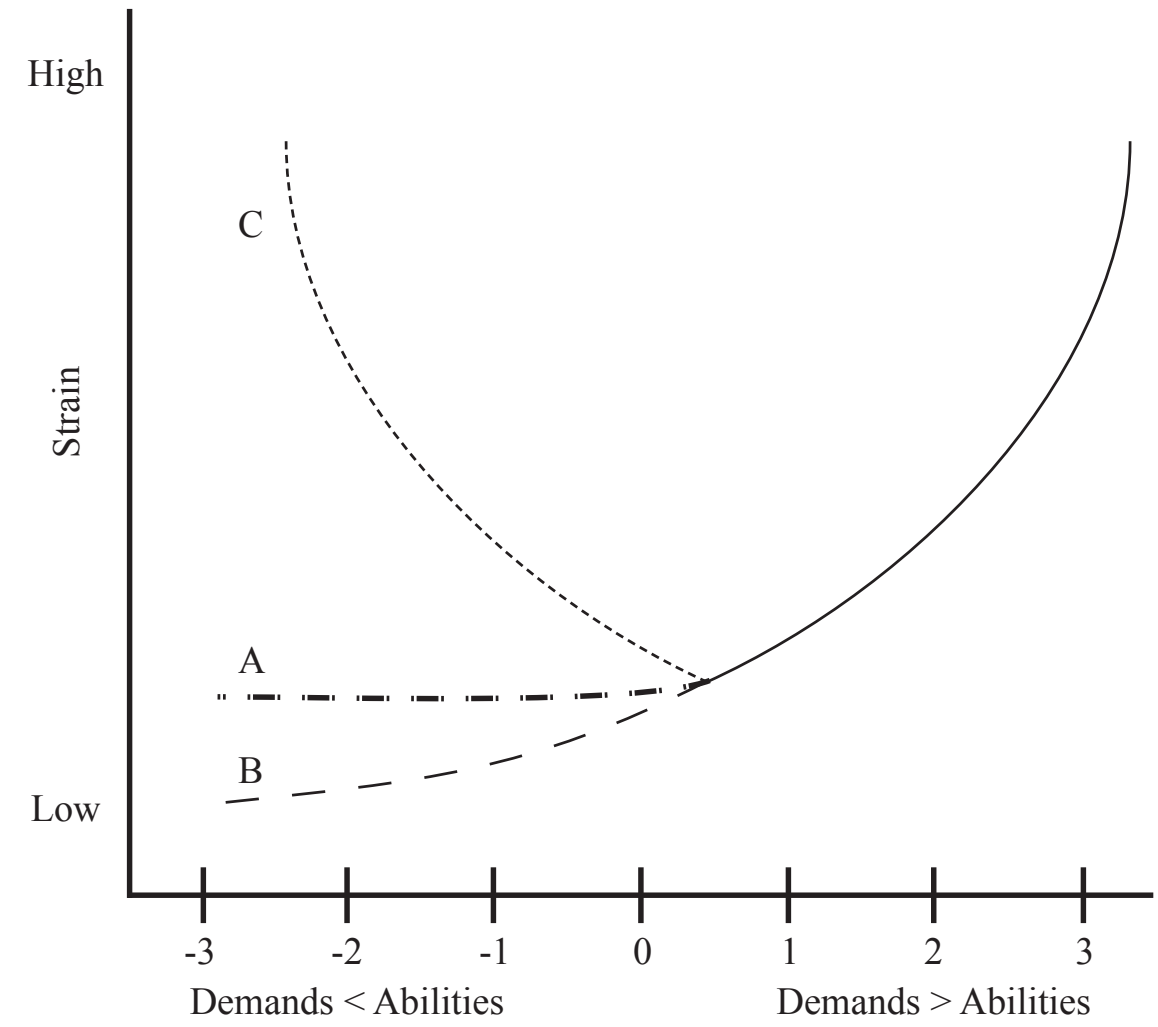

Figure 5. Three Forms of Excess in Demands-Abilities Fit illustrates three potential effects resulting from excess in abilities on strain: no effect due to one's abilities exceeding job demands (asymptotic, Curve A), reduction in strain due to one's excess in abilities (monotonic, Curve B), and increased strain due to one's excess in abilities (parabolic, Curve C).

\section{General Relationship between PE Fit and Strain}

In general, moderate-to-strong relationships between PE fit and psychological outcomes have been identified. A meta-analysis conducted by Bowling and Hammond (2008) estimated a moderately-strong relationship between PE fit and job satisfaction $(\hat{\rho}$ $=.46$ ), which was consistent with the same relationship identified in Kristof-Brown and colleagues' (2005) meta-analysis $(\hat{\rho}=.56)$. Kristof-Brown et al. (2005) also identified a moderate relationship between person-job fit and strain $(\hat{\rho}=-.28)$. In Kristof-Brown and colleagues' (2005) work, moderator analysis indicated type of fit to influence most fit- 
outcome relationships. In other words, the strength of fit-outcome relationships was dependent on if the study considered fit as an organization meeting employee's needs (i.e., needs-supplies fit), an employee meeting the organization's needs (i.e., demandsabilities fit), or similar values held by the employee and organization (i.e., supplementary fit). Needs-supplies fit type had the greatest impact on individual attitudes and behaviors, followed by demands-abilities and supplementary types of fit (Kristof-Brown et al., 2005).

Measurement strategy was also found as an important moderator of fit effects (Kristof-Brown et al., 2005). Specifically, direct measures that combine person and environment in single response items (e.g., "How well do your values fit with your organization's values?") generated stronger results than indirect measures that assess person and environment components separately (e.g., "How much autonomy do you prefer in your job?" and "How much autonomy is present in your job?"). However, critics of direct measures of fit have noted the susceptibility of single response items to common method bias, which may artificially attenuate the fit-outcome relationship (Kristof-Brown et al., 2005; Podsakoff, MacKenzie, Lee, \& Podsakoff, 2003).

\section{Measurement and Analysis Approaches to PE fit Research}

Because subjective PE fit is derived from the perceived person and environment, the cognitive comparison process is central to psychological theories regarding PE fit (Edwards et al., 2006). As such, how one perceives their fit with the environment and how PE fit is operationalized on the basis of these person and environment perceptions, has received much discussion and debate (Edwards et al., 2006; Edwards et al., 1998;

Kristof-Brown \& Guay, 2011; Schneider, 2001). The following discussion of approaches 
to PE fit research highlights several methods used in previous congruence research and concludes the review of PE fit theory.

\section{Atomistic, molecular, and molar approaches.}

In Edwards and colleagues' (2006) work, differences in how participants perceive fit were explored in a systematic review focused on types of measurement. As previously mentioned, the question of differences in congruence research results associated with measurement approach was raised in meta-analysis (Kristof-Brown et al., 2005) and has suggested different measurements of fit may tap into different psychological phenomena (Edwards et al., 2006). Measurement approaches highlighted by Edwards and colleagues (2006) included atomistic, molecular, and molar. An atomistic approach considered one's perceptions of person and environment as separate entities. An example of the atomistic approach would ask respondents two separate questions about a specific facet of their job, such as how much autonomy is present in their current job coupled with how much autonomy they prefer in a job. The indirect measurement of $\mathrm{P}$ and $\mathrm{E}$ factors was then combined to represent subjective PE fit. Separate measurement of P and E factors may reduce common response bias in that participants are not primed to think about discrepancy between factors, but to simply report in terms of what they want concerning a specific job characteristic and what they have of that characteristic in their job.

The molecular approach assessed perceived discrepancy by comparison between the person and environment while preserving the direction of their difference (i.e., indicating whether one is greater than or less than the other). An example of this measurement approach would be, "The amount of pay I receive is less than it should be" (Hollenbeck, 1989, p. 416). Items using this form of measurement frames a direction of 
discrepancy, possibly priming the individual to confirm or disconfirm using cognitive comparisons not truly associated with fit. For example, when asked if a job attribute is 'less than it should be,' a disparity in one's fit perceptions may inadvertently tap into equity comparisons to similar others, instead of comparing within one's preference for and supply of that job attribute. Of note, the molecular form of measurement retains directionality such that job characteristics that exceed personal preference are distinguishable from job characteristics that fall short of personal preference. Therefore, the conceptual relationship between atomistic and molecular is represented in the discrepancy between person and environment elements and described in the following theoretical equation:

$$
D=E-P
$$

where $D$ indicates the perceived discrepancy between perceived environment $(E)$ and perceived person $(P)$. Therefore, the molecular approach to measurement suggests that the perception of discrepancy is reduced when $\mathrm{E}$ and $\mathrm{P}$ are equal (Edwards et al., 2006).

And finally, the molar approach directly measures the perceived fit, match, or overall similarity between person and environment by asking the respondent a direct question about their fit perception (Edwards et al., 2006). The molar approach is typically seen in a global level of specificity in content dimensions without reference to comparisons. For example, an item that asks respondents to rate the fit between themselves and their workgroup would exemplify the molar approach. The molar approach also disregards directional differences and considers both negative and positive values as misfit. For example, whether misfit or incompatibility with one's workgroup 
was due to the group member having higher performance expectations of the group, or the group having higher performance expectations than the member was able to perform at, is lost when using a molar approach to fit measurement.

The relationship between molar and molecular approaches would be theoretically described as the difference between what perfect fit should be and how far off it is from that perfect fit. In other words, one's overall perception of fit can be described as 'perfect fit reduced by the discrepancy between E and P' (Edwards et al., 2006) and represented by equation:

$$
F=c-|D|
$$

where $F$ represents one's perception of overall fit, which is equated to one's psychological expectation of perfect fit $(c)$, lessened by the magnitude of discrepancy between person score and the environment score $(|D|)$. In Edwards et al.'s (2006) review, atomistic, molecular, and molar approaches to measurement of the same concept (i.e., PE fit) were only modestly related to each other, raising questions about the subjective meaning of PE fit and how it is represented in research.

\section{Difference scores approach.}

The use of difference scores to assess PE fit for job dimensions represents the first approach used in PE fit by the founders themselves. French, Caplan, and colleagues (Caplan et al., 1980; French et al., 1982) conducted the most comprehensive study designed to explicitly test PE fit theory to examine relationships between fit and strain. French and colleagues posited that strain would result from discrepancies between environment demands and one's abilities, or between one's needs and the environmental supply to meet those needs. French and colleagues' approach was developed from 
discrepancy theory in which the number of units separating $\mathrm{E}$ and $\mathrm{P}$ scores reflected the magnitude of discrepancy (Caplan et al., 1980; French et al., 1982; Harrison, 1976).

Operationalized as goodness-of-fit, measures of PE fit for each job-related dimension of interest were created by subtracting a participant's person (P) score (e.g., amount of each job characteristic they would prefer) from their environment (E) score (e.g., subjective appraisal of the job's supply of each characteristic). French and colleagues' (Caplan et al., 1980; French et al., 1982) approach was consistent with the atomistic level of measurement given separate measures for person and environment (Edwards et al., 2006). Content dimensions of interest, which included job complexity, responsibility for others, role ambiguity, and workload, were assessed at the facet level of specificity (Edwards \& Shipp, 2007). These difference scores mathematically represented the discrepancy between $\mathrm{P}$ and $\mathrm{E}$ measurements for each content dimension (Caplan et al., 1980; French et al., 1982).

Using a difference scores approach to measurement, a score of zero indicated perfect fit (i.e., E equals P). Negative scores indicated that a person's needs or abilities were higher than environmental supplies or demands, and positive scores indicated an environment value higher than the person value for that content dimension. Expected relationships between fit and strain included monotonic, asymptotic, and parabolic forms (French et al., 1982) as previously illustrated in Figures 4 and 5. To evaluate relationships assumed to be linearly related to strain (i.e., monotonic), the algebraic difference score called fit was used (E-P). As previously discussed, this fit-outcome relationship would indicate continued decrease in strain associated with excess in supply. For example, more job complexity present in one's job than preferred might be used by an employee to 
meet other needs (e.g., develop other skills or qualify for promotion opportunities) and through this carryover, have an association with reduced strain.

To assist in exploring and testing instances in which the fit-outcome relationship was curvilinear (i.e., asymptotic or parabolic), transformations of PE fit were created. A parabolic fit-outcome relationship exists when increasing absolute magnitude of $\mathrm{P}$ and $\mathrm{E}$ scores are related to increased strain. For example, both too much workload (relative to one's preference) and too little workload may be associated with increased strain. An absolute value transformation called poor fit $(|\mathrm{E}-\mathrm{P}|)$ was used to test a parabolic fitoutcome relationship in the French and colleagues' (1982) study. The absolute value transformation produced a scale of the magnitude of poor fit with no distinction made as to whether the discrepancy resulted from an environmental deficit in supply or an oversupply of the content dimension. In other words, the absolute value transformation was used to assist in modeling underlying curvilinear relationships by using a linear equivalent. Of note, a significant curvilinear effect may relate to different strains from one side of the curvilinear relationship to the other. To elaborate using the previous example, too much workload may result in strain outcomes such as anxiety and sleeplessness; whereas too little workload may result in strain outcomes such as boredom and lack of engagement.

To explore an asymptotic effect, PE fit transformations were created as left- or right-censored difference scores. Censored transformations were called excess and deficiency, respectively, which results in strain showing a linear representation of the existing asymptotic relationship (see Figure 6). Specifically, if strain was hypothesized to increase in magnitude only on one side of perfect fit (i.e., either greater than or less 
than perfect fit) while remaining unaffected when discrepancy in PE fit increased on the other side of perfect fit, scores on the side thought to have no relationship to strain were recoded to the value of perfect fit.

Excess measures were created to model instances in which the environment scores exceeded the person scores (i.e., E more than P), such as when one's skills or abilities were not adequate to meet the demands of the job. Left-censored transformation was accomplished by setting all negative values of the $\mathrm{E}-\mathrm{P}$ difference to zero, which enabled detection of relationships between PE fit and strain where E was greater than P (i.e., when supplies exceeded needs or demands exceeded abilities). Conversely, deficiency measures were created to model a relationship in which the environment was deficient in matching one's needs (i.e., E less than P), such as when one's preference for promotion opportunities in a job was not supplied with such opportunities for advancement. For the deficiency or right-censored transformation, all positive scores were reassigned to zero (i.e., the value for perfect fit). 


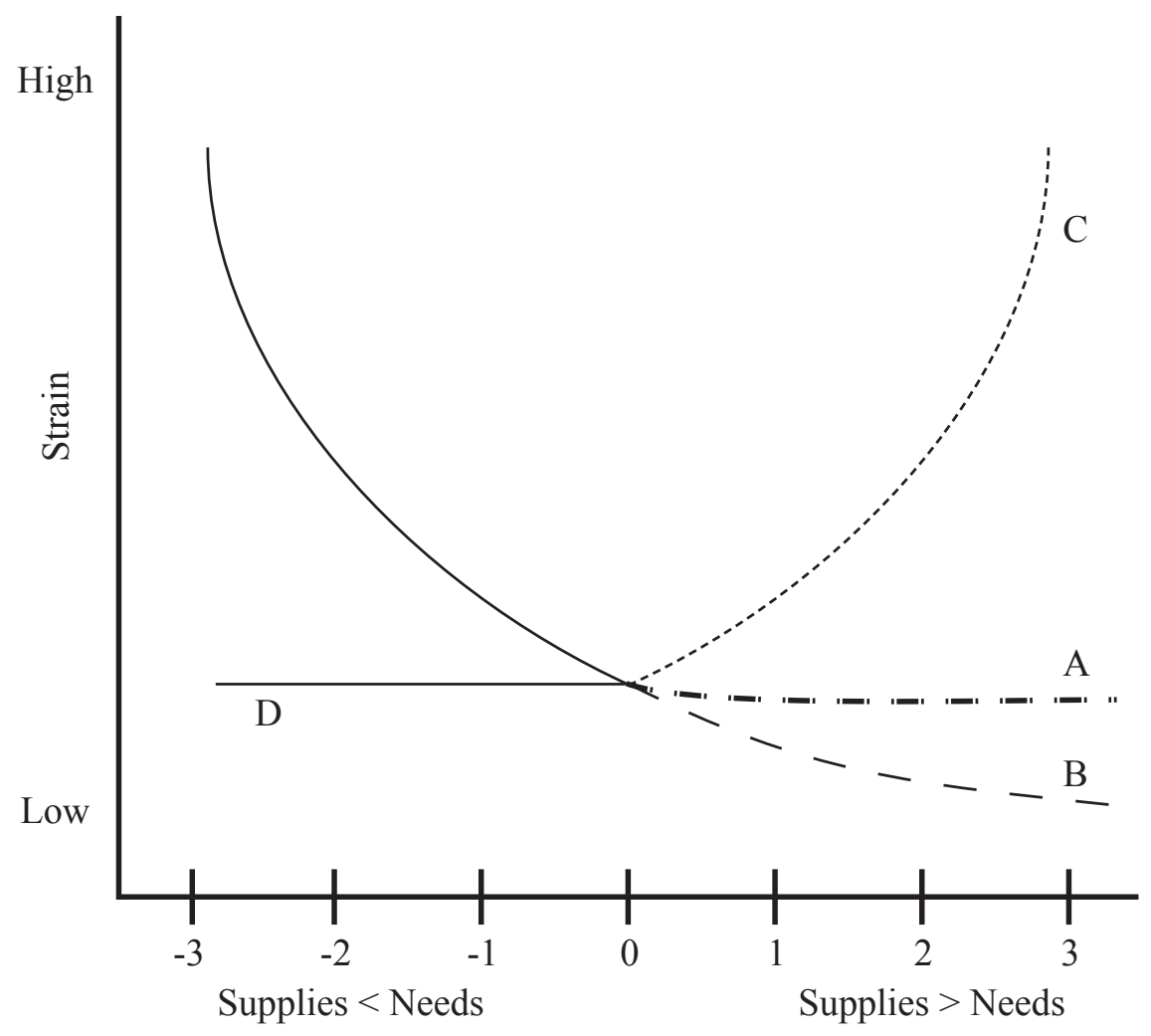

Figure 6. Transformation for Asymptotic Effects. This figure illustrates the excess transformation, when supplies exceed one's needs. To model asymptotic effects (Curve A) with a left-censored transformation, scores on the left side of the figure (assumed to be unrelated to strain) were recoded to perfect fit (i.e., zero) as seen in Line D.

To explore all potential relationship forms between fit and strain, each transformation was evaluated for each job characteristic dimension. Table A1 (in Appendix A) shows correlations between selected strain outcomes with job fit transformations of fit, deficiency, excess, and poor fit. In evaluating strength of associations, French and colleagues (1982) considered the strongest significant correlation between each strain and PE fit transformation indicative of its form of excess (i.e., monotonic, asymptotic, or parabolic). For example, a curvilinear relationship was predominant for the job characteristic of job complexity. Correlations with job 
complexity transformations indicated that job dissatisfaction, workload dissatisfaction, boredom, and depression increased when the job was either too simple or too complex (French et al., 1982).

Relationships between PE fit (defined by difference scores and their transformations) and strain outcomes were also tested by examining the incremental variance explained by $\mathrm{PE}$ fit transformations after controlling for $\mathrm{P}$ and $\mathrm{E}$ main effects (Caplan et al., 1980; French et al., 1982). Difference scores transformations representing curvilinear effects, (i.e., deficiency, excess, poor fit) often found incremental variance explained after controlling for main effects of $\mathrm{P}$ and $\mathrm{E}$. For example, dimensions of job complexity and role ambiguity were found to have a parabolic relationship with psychological strains of job dissatisfaction and boredom. In other words, misfit in job complexity or role ambiguity, as indicated by discrepancies from perfect fit in either direction, were related to increased dissatisfaction and boredom for employees (French et al., 1982).

Overall, major findings from the French and colleagues' (1982) study included 1) misfit was frequently associated with increased strain, 2) the relationship between misfit and strain was often parabolic, and 3) models using a parabolic relationship often accounted for significant variance beyond that explained by E and P main effects (Edwards \& Harrison, 1993).

\section{Gaps/limitations with difference scores.}

Most studies published between 1960 and 1990 operationalized PE fit using methods similar to difference scores transformations (Edwards, 1991). Despite their widespread use, difference scores were prone to several methodological problems 
associated with the difference score process of combining conceptually distinct constructs into a single score (Cronbach, 1958; Johns, 1981; Nunnally, 1962). The current section discusses methodological issues inherent to difference scores and their transformations.

Reliability and systematic correlation. Because of limitations in measurement of non-observable values (e.g., one's preferences or subjective appraisal of the environment), a reliability index is used to quantify how well the measurement assesses the concept of interest (Cohen, Cohen, West, \& Aiken, 2003). The difference between a calculated reliability coefficient and perfect reliability associated with a true (i.e., error free) score is an index of the relative amount of measurement error. Low reliability of measurements adversely affects the ability to statistically detect relationships between variables.

Reliability of a difference score tends to be less than the average reliability of its person and environment components (Johns, 1981). Specifically, reliability of a difference score is based on the reliability of individual $\mathrm{P}$ and $\mathrm{E}$ components, their variances, and the correlation between $\mathrm{P}$ and $\mathrm{E}$ (see Equation 3).

$$
r_{E-P}=\frac{\sigma_{E}^{2} r_{E E}+\sigma_{P}^{2} r_{P P}-2 r_{E P} \sigma_{E} \sigma_{P}}{\sigma_{E}^{2}+\sigma_{P}^{2}-2 r_{E P} \sigma_{E} \sigma_{P}}
$$

Reliability for a difference score will equal the average reliability of its components only when the correlation between components is zero. Because $\mathrm{E}$ and $\mathrm{P}$ assess meaningfully related constructs (e.g., one's preference for and supply of the same job characteristic) and that responses are often from the same source (e.g., employee's subjective perception of these components), a correlation of zero between them is not 
likely (Johns, 1981). When P and E components comprising a difference score are correlated with each other, the outcome may involve substantial error due to the attenuated reliability of both measures. In other words, a non-zero correlation between the components of the difference score amplifies the reliability of both measures, resulting in substantial error (i.e., unreliability) in the difference score itself. Difference scores tend to be systematically correlated with their component parts, which contributes to the potential for artifactual relationships (Johns, 1981).

Untested constraints. Difference scores implicitly impose constraints upon the relationship between component measures and the outcome. For example, using the algebraic difference transformation (i.e., E - P), the equation to examine fit-outcome relationships would be:

$$
Z=b_{0}+b_{1}(E-P)+e
$$

Expanding Equation 4 to components:

$$
Z=b_{0}+b_{1} E-b_{1} P+e
$$

Equation 5 shows the implied constraint in regression coefficients for $\mathrm{E}$ and $\mathrm{P}$ to be equal in magnitude, but opposite in sign.

In Edwards' (1991) review of congruence research, another difference score approach for modeling parabolic effects for both sides of fit $(\mathrm{E}<\mathrm{P}$ and $\mathrm{E}>\mathrm{P})$ used the squared difference transformation $\left[(\mathrm{E}-\mathrm{P})^{2}\right]$. Constraints imposed by this transformation are shown in Equations 6 through 8. First, the equation to examine curvilinear fitoutcome relationships using a squared difference transformation would be:

$$
Z=b_{0}+b_{1}(E-P)^{2}+e
$$


Expanding Equation 6 to components:

$$
Z=b_{0}+b_{1} E^{2}+-2 b_{1} E P+b_{1} P^{2}+e
$$

Note that the difference score presented in Equation 6 contains curvilinear and interactive terms without appropriate lower order terms (Cohen, 1978; Edwards \& Parry, 1993).

Including null coefficients for lower order terms yields:

$$
Z=b_{0}+0 E+0 P+b_{1} E^{2}+-2 b_{1} E P+b_{1} P^{2}+e
$$

Equation 8 identifies a) the missing lower order terms (E and P), b) the forced equality of regression coefficients for $\mathrm{E}^{2}$ and $\mathrm{P}^{2}$ terms, and c) a negative coefficient twice as large as that of $\mathrm{E}^{2}$ and $\mathrm{P}^{2}$ terms, for the EP product term.

Dimensional reduction. Person-environment fit theory establishes E and P as distinct constructs, with subjective PE fit as the relationship between subjective person and subjective environment. Therefore, the effect of person-environment fit is essentially a three-dimensional relationship between two predictor components and an outcome. Specifically, as distinct elements, the relationship between $\mathrm{E}$ and $\mathrm{P}$ with strain is essentially in three dimensions, with $\mathrm{E}$ and $\mathrm{P}$ on two perpendicular horizontal axes and strain on the vertical axis. By reducing this inherently three-dimensional surface, conceptual distinctions between $\mathrm{E}$ and $\mathrm{P}$ are lost, the potential complexity of their joint effects on strain is oversimplified, and further examination of the relationship is prevented (see Figure 7). 


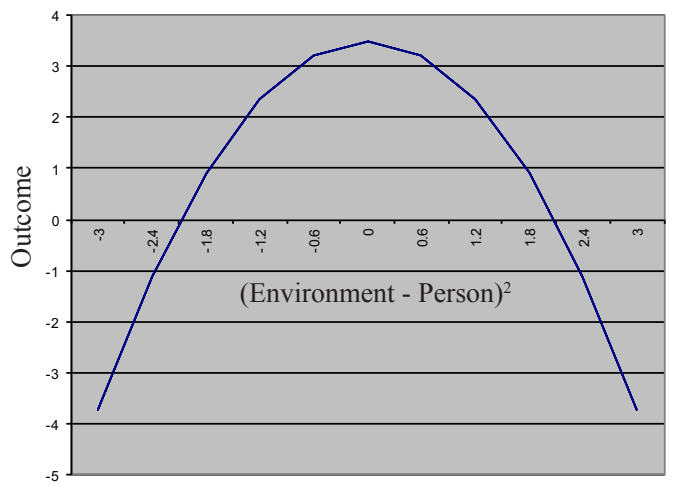

b.

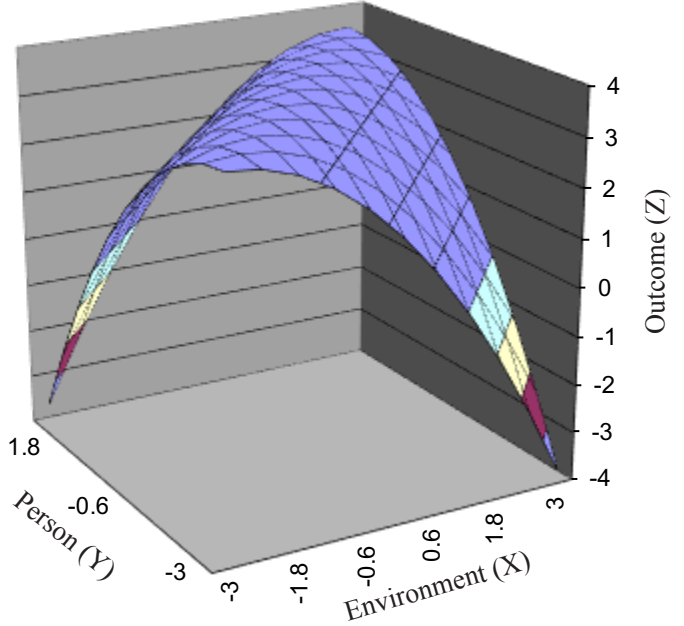

Figure 7. Dimensional Reduction. Visual comparison between (a) two-dimensional squared difference function and (b) corresponding three-dimensional square difference function.

Confounded effects. As difference scores estimates are calculated from component measures reduced to a single coefficient, substantial differences in the effects of the components are confounded. For example, if only E or P was significantly correlated with strain, the corresponding fit measure may have reflected the influence of only one component, and results relating fit to strain would be spurious (Wall \& Payne, 1973). To illustrate, consider Equation 5. Would the fit-outcome relationship modeled in Equation 5 be due to the positive $b_{1}$ coefficient for E, or the negative $b_{1}$ coefficient for P? As these components are combined, their individual contribution cannot be untangled and interpreted.

Ambiguous interpretation. While a difference score may seem to represent equal contributions in opposite directions, in actuality, variance of difference scores is a function of the variances and covariance of the component measures:

$$
\sigma_{(E-P)}^{2}=\sigma_{E}^{2}+\sigma_{P}^{2}-2 \sigma_{E P}
$$


Because greater weight is given to the component measure with larger variance, $\mathrm{E}$ and $\mathrm{P}$ will account for equal amounts of variance in their difference score only when their component variances are equal. As environment and person components are distinctly different, their variances are likely to differ as well. Information used to interpret weight of components is discarded from a difference score, leaving interpretation of component contribution to implicit assumption of equal weights (Edwards, 2002).

Additionally, squared differences are interpreted as symmetric measures of congruence, given that positive and negative differences are treated the same. Interpretation of the squared difference transformation implies that both positive and negative scores contributed to the difference, which might not always be the case. For example, people often report receiving less reward or compensation than desired. When creating a difference score in which desired amount is subtracted from received amount, predominantly negative scores would result, which are misinterpreted as symmetric indices of need fulfillment.

\section{Polynomial regression approach.}

Methodological limitations associated with difference scores are avoided by using polynomial regression that includes separate component scores of $\mathrm{P}$ and $\mathrm{E}$ in the model. Specifically, it addresses congruence as the correspondence between component measures in two-dimensional predictor space (Edwards, 2002) rather than the onedimensional (i.e., scalar) space of difference scores. Recall that for difference score approaches, a difference score of zero was considered perfect fit as both E and P components were equal. Perfect fit using polynomial regression approach is not a single point in the PE space, but a line of congruence in the two-dimensional PE space such that 
E and $\mathrm{P}$ components are equal (see Figure 8). The polynomial regression approach to congruence preserves the magnitude and direction of components to better understand both congruence and incongruence (i.e., fit and misfit).

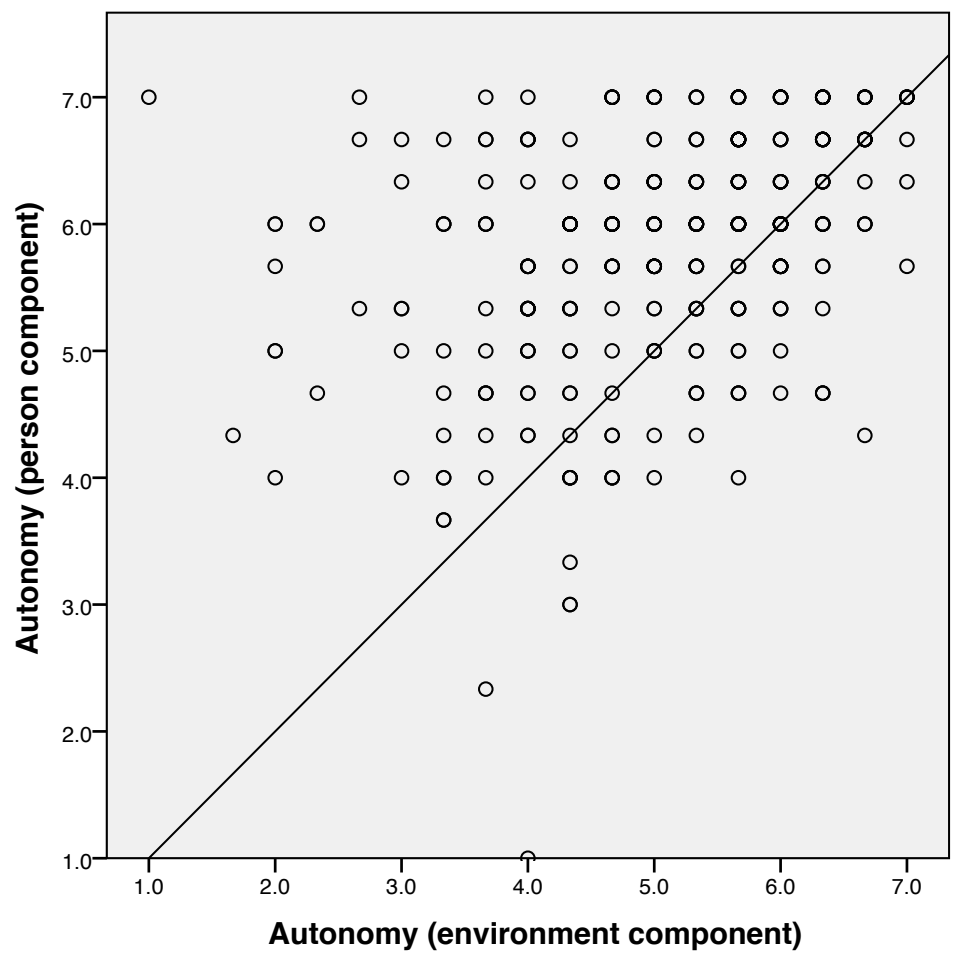

Figure 8. Scatterplot of Predictor Space. Solid line represents congruence or agreement between person and environment components of autonomy (i.e., when scores were equal to each other).

Equation (10) uses a polynomial regression approach, in which components of the difference were entered as separate predictors with their constituent lower order terms included (Aiken \& West, 1991; Edwards, 2002).

$$
Z=b_{0}+b_{1} E+b_{2} P+b_{3} E^{2}+b_{4} E P+b_{5} P^{2}+e
$$

Equation 10 includes all component terms, allows the model to fit the data without a priori constraints, and represents a response surface in three dimensions. 


\section{Response surface methodology.}

A response surface is the visual, three-dimensional representation of the joint effects of two predictors on an outcome (Box \& Draper, 1987; Edwards, 2002; Shanock et al., 2010). While response surface methodology has typically been applied to multisource feedback discrepancies, it provides more explanatory potential than difference scores or traditional moderated regression analyses (Shanock et al., 2010). Specifically, standard moderated regression avoids several difference scores pitfalls by including lower order terms and not constraining coefficients to be equal. From standard moderated regression with main and interactive terms (i.e., E, P, and EP), polynomial regression analysis that includes the higher order quadratic terms (i.e., $\mathrm{E}^{2}$ and $\mathrm{P}^{2}$ ) improves the model's ability to detect curvilinear effects in the fit-outcome relationship.

Starting with groundwork developed by Edwards and colleagues (Edwards 2002; Edwards \& Harrison, 1993; Edwards \& Parry, 1993) research utilizing polynomial regression with response surface analysis has increased. Examples include congruence between organizational and departmental commitment to safety affecting employee safety behaviors (Huang, Lee, McFadden, Rineer, \& Robertson, 2017), congruence between team and leaders' perceptions of learning affecting performance (Tafvelin, von Thiele Schwarz, \& Hasson, 2017), leadership-member relationship quality affecting employee stress (Harris \& Kacmar, 2006), and needs-supplies fit in the content dimension of leadership style affecting employee attitudes and counterproductive work behaviors (Rupprecht, Kueny, Shoss, \& Metzger, 2017).

In 1999, Edwards and Rothbard used polynomial regression with response surface analysis to examine needs-supplies fit for several work-related attributes on well-being 
outcomes. Specifically, needs-supplies perceptions were assessed for content dimensions of autonomy, interpersonal relations, role security, and segmentation (between work and family) to examine how fit in each of these dimensions impacted satisfaction and physical symptoms. While general misfit between one's preferences and perceptions was found detrimental to employee well-being, nuances in the fit-outcome relationship were also identified. Specifically, autonomy misfit differentially related to satisfaction depending on if autonomy was oversupplied (i.e., excess) or undersupplied (i.e., deficient) in the job. While satisfaction was reduced when needs and supplies did not match in general, this reduction in satisfaction was not as sharp when the discrepancy favored supplies. In other words, when the amount of autonomous decision-making latitude provided at work was above employee's preferred amounts, higher satisfaction levels were reported than when employees' preferred amount of autonomy was not provided at work (Edwards \& Rothbard, 1999).

A detailed view of the fit-outcome relationship was not discernable using difference scores approach because of lost information (i.e., due to reducing components to one value). Using polynomial regression with its resulting response surface, all information is retained to examine the effects of fit for all values of the person and environment fit components on an outcome. Modeling the response surface is accomplished by functions of the coefficients that reveal other specific effects of substantive interest. For example, while compatibility between one's preference and actual presence of job attributes is expected to maximize positive outcomes, these outcomes have been found highest when the compatibility match referred to a high preference/high presence of a job attribute. The hypothetical illustration between decision 
latitude fit and satisfaction presented in Figure 9 illustrates a response surface in which high preference for decision latitude is matched with high presence of decision latitude in the job, and associated with higher levels of satisfaction.

Additionally, degree and direction of misfit (i.e., discrepancy between environment and person) may have differential relationships with an outcome. As illustrated in Figure 9, the marked decline in satisfaction levels related to the degree of discrepancy between preferred and present decision latitude are apparent in the negative curvature along the line of misfit (i.e., dashed line). The direction of misfit refers to a differential relationship with an outcome due to oversupply or undersupply of the job attribute. As illustrated, when misfit was in terms of oversupply of decision latitude, satisfaction levels were not as low as when decision latitude was undersupplied. 


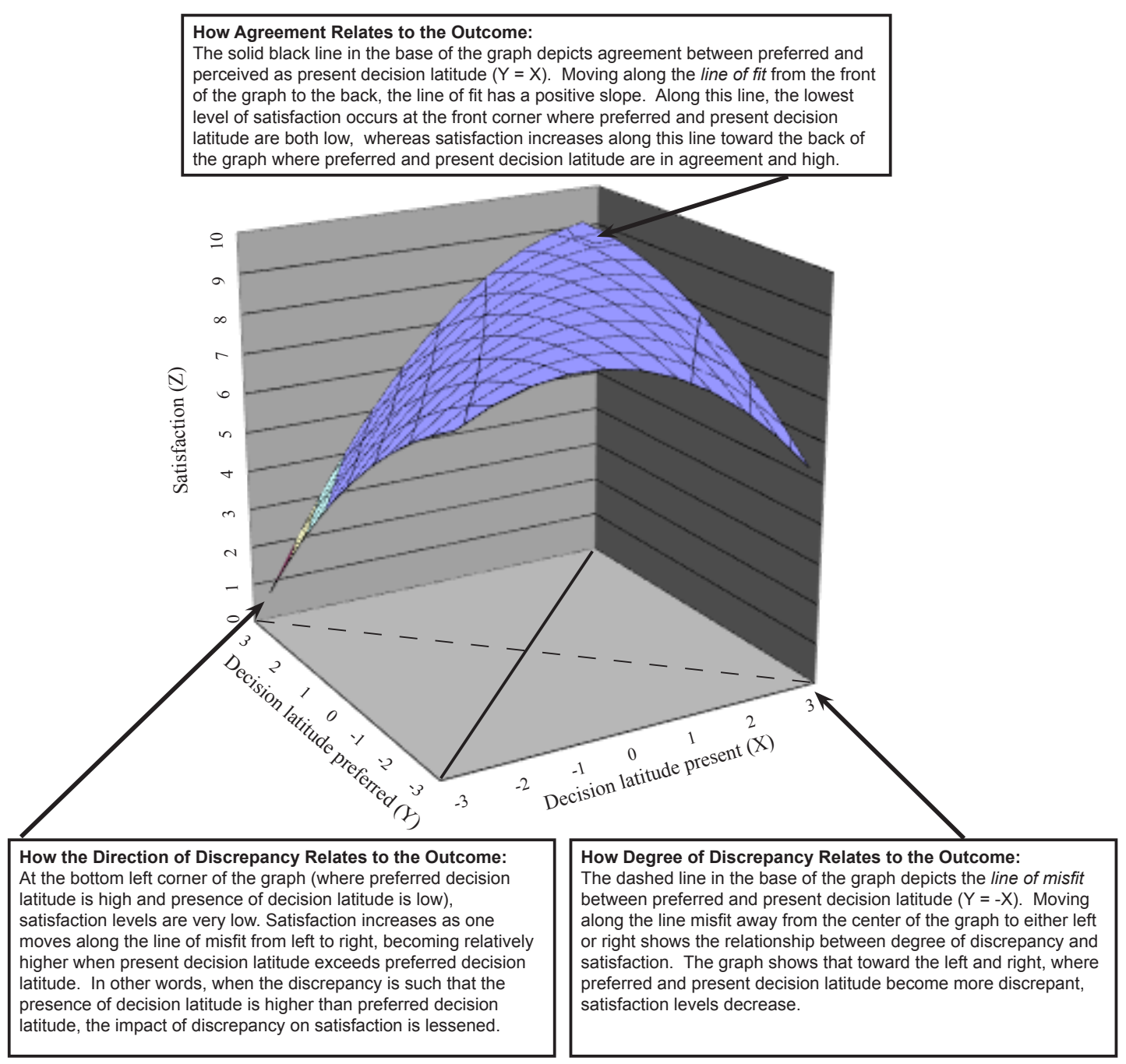

Figure 9. Hypothetical Response Surface illustrates satisfaction as related to preferred and present decision latitude.

Results from fit research studies (Edwards, 2002; Edwards \& Harrison, 1993, Edwards \& Parry, 1993; Edwards \& Rothbard, 1999) using polynomial regression with response surface analysis have been generally consistent with meta-analytic estimates found by Kristof-Brown and colleagues (2005). However, information gained through inspection of the response surface has revealed more complicated fit-outcome relationships than reported by difference scores or moderated regression (Kristof-Brown \& Guay, 2011; Shanock et al., 2010). For example, in Edwards and Harrison's (1993) 
comparison of approaches, response surface analysis identified details in the job complexity fit-boredom relationship not recognized by prior methodological approaches. Specifically, boredom was optimally minimized when job complexity in the environment slightly exceeded one's preferred level of job complexity, which was particularly effective at low preferred/low present agreement of job complexity fit (Edwards \& Harrison, 1993).

In summary, the fit-outcome relationship is complex, and this complexity can be described better using response surface analysis. While these differential effects within the fit-outcome relationship were presaged by original PE fit theorists (Caplan et al., 1980; French et al., 1982; Harrison, 1978), this detail in the nature and nuance of the fitoutcome relationship was indistinguishable using prior methods (Edwards 2002). Details regarding identification of fit and misfit as they relate to an outcome are discussed in Chapter III.

\section{JCT as Supplemental Theoretical Source for PE Fit Theory}

As previously noted, $\mathrm{PE}$ fit theory describes the mechanism of fit but requires content dimensions supplied from other theoretical sources. The focus of the dissertation is the impact of job fit and job misfit. Therefore, job characteristics variables were chosen based on their prevalence and application in the workplace. Job characteristics theory by Hackman and Oldham $(1976,1980)$ was used as the theoretical framework for these variables. In other words, PE fit theory was used to explain the mechanism of how misfit between one's preferences and job attributes affect outcomes and JCT was used as the supplemental theoretical base for content dimensions of interest. While JCT was

originally developed as a work motivation theory, the dissertation uses JCT to provide 
guidance regarding job characteristics associated with an enriched job and related to beneficial outcomes. Beneficial outcomes that result from one's fit with their job across job characteristics, are evaluated from this dual-theoretical base for the dissertation. The following discussion includes theoretical development of the Job Characteristics Model and concludes with gaps and limitations of JCT.

\section{Development of JCT.}

Hackman and Lawler (1971) began the foundational work used by JCT in their conceptual development of job characteristics thought to enhance work motivation. Their theoretical underpinning hinged on needs fulfillment and expectancy theories (Alderfer, 1969; Lewin, 1943; Maslow, 1943; Vroom, 1964). Basically, behaviors are driven by fulfillment of needs, starting at existence needs (physiological, safety) and moving up in levels through relatedness (love), and growth (esteem, personal life goals, reaching one's potential). Hackman and Lawler (1971) outlined the connection between expectancy theories and needs fulfillment, towards work motivation in five points:

- Outcomes that satisfy physiological or psychological needs (or lead to outcomes that satisfy needs) become valued by individuals.

- Valued outcomes (both intrinsic and extrinsic) become incentives, as individuals believe a course of action will result in obtainment of those valued outcomes.

- Strategic job design, such that individuals can best meet their own needs by working effectively towards organizational goals, generates conditions in which employees work diligently towards those goals.

- Attainment of higher order needs satisfaction may actually increase the strength of one's drive for needs fulfillment (Alderfer, 1969). Therefore, the opportunity 
for development of continuing or increasing motivation is possible when higher order needs are engaged.

- Individuals desiring of higher order needs satisfaction will experience such satisfaction as they learn that they have, by their own efforts, accomplished something that they believe is personally worthwhile.

Using these propositions, Hackman and Lawler (1971) suggested that characteristics of jobs could be designed to establish conditions that facilitate the intrinsic motivation of employees who desire higher order needs satisfaction.

\section{Job Characteristics Model.}

Job characteristics theory posited that employees experience work motivation to the extent that they learn that they have performed well on a task that is meaningful to them. Specifically, Hackman and Oldham $(1976,1980)$ posited that five core job characteristics influence three critical psychological states, which then impact personal and work outcomes such as work effectiveness, absenteeism, work motivation, growth satisfaction, and overall satisfaction (see Figure 10). 


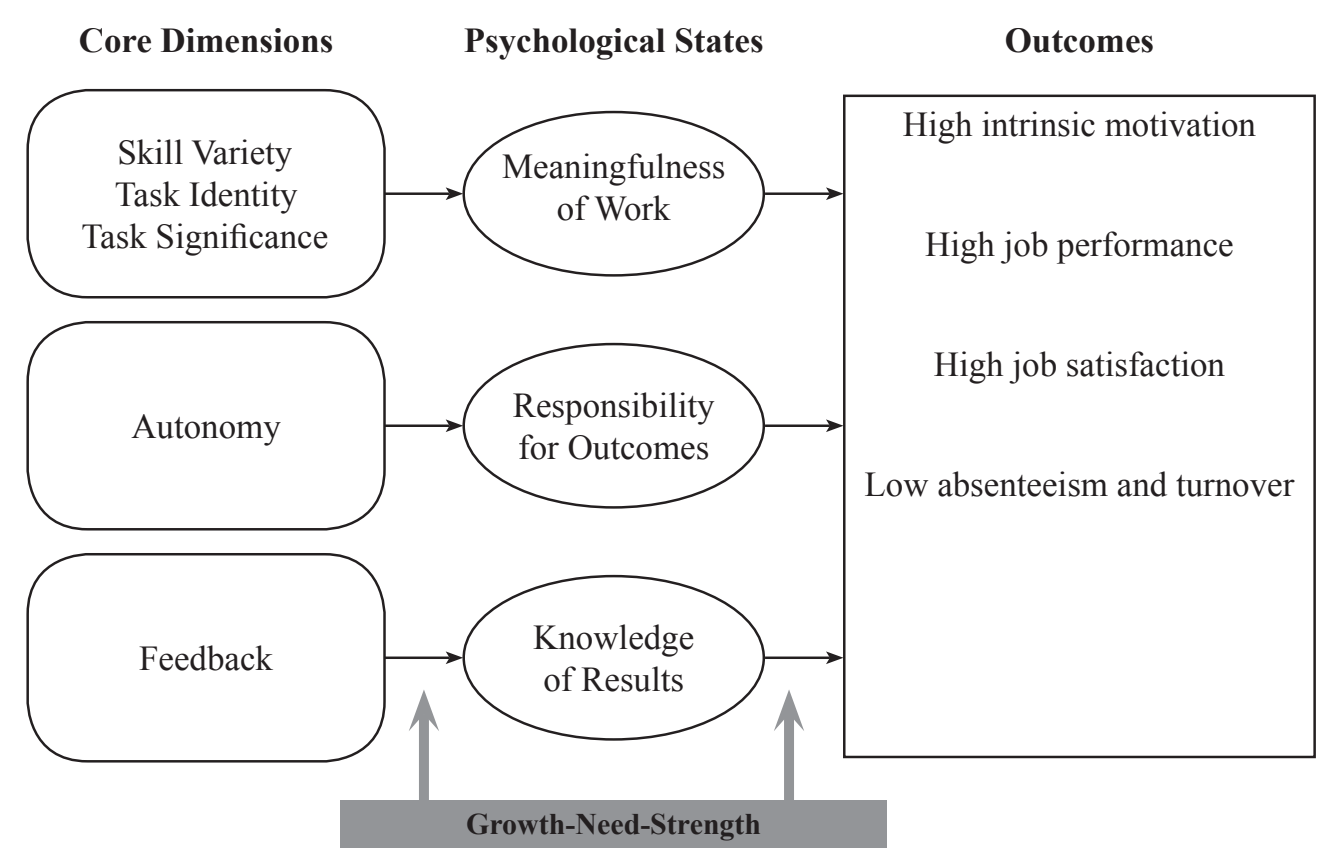

Figure 10. Job Characteristics Model

\section{Core job characteristics.}

Job characteristics refer to the tasks, work activities, or other operations of a job. Some job characteristics have been associated with higher motivation and performance, such as the ability to make decisions about how the work should be completed or the use of innovative or creative thinking to successfully perform at a job. According to JCT, the following job characteristics are posited to increase work motivation.

Skill variety refers to the degree to which a job requires the performance of a wide range of operations and/or the degree to which employees must use a variety of equipment and procedures in their work. Skill variety has also been referred to as task variety, and job or task complexity (Griffin et al., 2012). In the context of JCT, jobs high in variety are more likely to utilize skills or abilities valued by the individual, contributing to its relationship with internal work motivation (Fried \& Ferris, 1987). 
Specifically, the opportunity to use valued skills is posited to meet individual's higher order needs, such as needs for recognition/appreciation, needs for meaningfulness in work. As such, increased skill variety has commonly been considered a motivator rather than a stressor (Ilgen \& Hollenbeck, 1991). However, the study of misfit between one's preference for skill variety and the environment's supply of skill variety conceptualizes the job characteristic as a potential stress.

Task identity refers to the extent the job requires completion of an entire or whole piece of work, such that results of efforts can be clearly identified by the employee. Task identity is present in a job that involves a relatively whole piece of work, such that an employee perceives they have principally contributed to a production or accomplishment of consequence. It is characterized by (a) a clear cycle of perceived closure, or a distinct sense of beginning and ending of a process, (b) high visibility of the transformation process to the employee, (c) high visibility of the transformation in the finished product, and (d) a transformation of considerable magnitude. According to Hackman and Oldham $(1976,1980)$, the possibility to stay involved in a work process throughout its stages with visible outcomes is more interesting than performing only a part of a job or incomplete tasks. Individuals fundamentally prefer to see a task from start to finish and see the whole completed work as this provides a sense of meaningfulness (Ryan \& Deci, 2000). Task identity may be especially relevant to employees with high needs for developing and using their competence, contributing to their experienced meaningfulness (Hackman \& Lawler, 1971).

Task significance is the extent to which the job provides opportunities to have a positive impact on the well-being of other people. As an antecedent to experienced 
meaningfulness, task significance helps employees perceive their work as worthwhile and valuable. Task significance also takes on relational significance as it makes salient the connections between emergency responders and how their actions impact others. In other words, task significance strengthens employees' perceptions of impact on others and enables employees to feel valued and appreciated by those they help (Grant, 2008; Grant, Fried, \& Jullierat, 2011).

Autonomy refers to the degree a job allows freedom and independence in how the work is executed, such as in having latitude in scheduling their work, selecting the equipment they will use, and deciding on procedures to be followed. To the extent that a job has high autonomy, outcomes increasingly depend on one's own abilities and decisions rather than a given set of directions. Autonomy is posited to stimulate a sense of responsibility for the successes or failures that occur on the job and increase job satisfaction (Hackman \& Lawler, 1971; Hackman \& Oldham, 1976, 1980).

Feedback from the job refers to the degree to which carrying out work activities required by the job provides an employee with direct and clear information about their performance effectiveness. Job-based feedback is based in the knowledge of results literature, in which the quality of feedback has been found related to performance criteria (Annett, 1969). Compared to agent-based feedback (e.g., from supervisor or coworkers), feedback mechanisms that are part of the work itself provide the most proximal information about performance and are directly characteristic of the job. Also referred to as task or task-based feedback, job-based feedback provides information in a timely basis and is perceived as more psychologically safe than agent-based feedback. 
As previously stated, PE fit theory provides a framework to understand the mechanism of need-supplies fit in a workplace context. Job characteristics theory provided the theoretical framework for the dissertation's job characteristics dimensions. In other words, the five core job characteristics of JCT were used to provide necessary content dimensions for PE fit congruence research. Remaining elements of the Job Characteristics Model are outlined below.

\section{Critical psychological states.}

As previously stated, JCT was used as the necessary supplemental contributor of content dimensions for utilizing PE fit. However, a brief review of JCT assists in understanding how and when job attributes are expected to facilitate beneficial organizational and personal outcomes. While not included in the dissertation, the conceptual core of JCT involved three critical psychological states posited to mediate the relationship between the core job characteristics and personal and work outcomes. According to the theory, employees needed to experience all three critical psychological states to achieve the outcomes proposed. Specifically, when employees experienced these states, they felt good about themselves and their work, and therefore were reinforced through positive feelings to keep performing well.

Experienced meaningfulness refers to the degree employees experienced their job as intrinsically meaningful, worthwhile, and valuable. Three core job characteristics contribute to experienced meaningfulness: skill variety, task identity, and task significance. Experienced responsibility for work outcomes refers to the degree employees feel personally accountable or responsible for the results of their work, which is influenced by the core job characteristic of autonomy. Knowledge of results refers to 
the degree employees understand how effectively they are performing at their jobs and is influenced by the core job characteristic of feedback. When these three psychological states are present, the overall potential of the job to prompt internal work motivation would be at its highest. Similar to Vroom's (1964) equation of motivational force, Hackman and Oldham $(1976,1980)$ formulated the motivating potential score (MPS) index as the degree to which a job is likely to prompt favorable personal and work outcomes. The MPS is calculated using core job characteristics associated with each psychological state as follows:

$$
M P S=\frac{\text { skill variety }+ \text { task identity }+ \text { task significance }}{3}(\text { autonomy })(\text { feeback })
$$

Of note, a low score in autonomy or feedback would substantially reduce the MPS for a particular job (Kulik, Oldham, \& Hackman, 1987). However, job characteristics that influence experienced meaningfulness are able to offset each other in a compensatory fashion to maintain a high MPS even if one characteristic is low.

\section{Moderated by employee's growth-need-strength.}

Realizing from previous work (Hulin \& Blood, 1968; Turner \& Lawrence, 1965) that individual differences must be taken into account simultaneously with job characteristics, Hackman and Lawler (1971) had used a 12-item measure to assess individuals' desire for satisfaction of higher-order needs. In development of JCT, the effects of individual differences in the form of growth-need-strength (GNS) were included to serve this purpose (Hackman \& Oldham, 1976). Specifically, the measurement of GNS involved respondents indicating their preference between two job attributes, one related to the higher order need of growth (i.e., autonomy, variety) and the 
other related to a different, non-growth-related attribute (e.g., pay, security, social opportunities, teamwork preference). Growth-need-strength reflected the degree to which an individual valued higher order needs (e.g., opportunities for personal growth and development) and was posited to moderate the relationships between core job characteristics, critical psychological states, and outcomes (Hackman \& Oldham, 1975). Basically, individuals who have a high need for personal growth and development were expected to respond more positively towards enriched jobs and better able to experience the critical psychological states. The moderating effect of higher level needs was confirmed for general, growth, and work satisfaction outcomes in meta-analyses conducted by Spector (1985).

Criticisms regarding the Job Characteristics Model include dimensionality of the core characteristics and the psychological states (Fried \& Ferris, 1987; Idaszak \& Drasgow, 1987). Also, the moderating effect of GNS has not been supported as variance in both the high and low GNS groups was because of sampling error, range variation, and measurement error. Some concerns regarding the utility of JCT have been raised because stronger relationships have been found between job characteristics and affective outcomes compared to relationships to behavioral outcomes, and also because of limited accuracy between employee's perceptions of job characteristics and objective characteristics (Kulik et al., 1987). In other words, what benefit can be reasonably expected from redesign efforts given employees' inaccurate perception of the objective environment and limited relationships to employees' actual behavior? While these are valid criticisms of self-report data, some information is best provided by the incumbent (Spector, 1994). Especially, as PE fit theory has identified subjective fit as the primary 
factor in strain outcomes (French et al., 1982; Harrison, 1978), the dissertation also used employee self-report of their perceptions of job characteristics.

\section{Gaps and limitations of JCT.}

Job characteristics theory has two primary weaknesses. First, while individual differences are included, they are represented by a composite reflecting personality trait preference for growth (i.e., GNS), instead of assessing one's preference for each job characteristic. For example, JCT posits that individuals will be more motivated by jobs high in skill variety. However, for individuals who prefer simple, straightforward tasks, a high level of skill variety may be perceived as stressful or an overload to their work responsibilities.

Secondly, JCT assumes a monotonically increasing relationship between job characteristics and outcomes. In other words, more of each job characteristic is always better. As discussed later in the dissertation, curvilinear relationships between job characteristics and individual outcomes have been identified (e.g., Chung-Yan, 2010), which discredit the 'more is better' assumption. These limitations can be addressed by a) considering one's preference for each job characteristic in its own right and b) considering potential curvilinear effects. Evaluating the impact of PE fit for these job characteristics dimensions using polynomial regression addresses these limitations.

Specifically, the dissertation recognized that individuals may have unique preferences for each job characteristic and posited that misfit on job characteristics dimensions impact outcomes, not the environmental supply of job characteristics in isolation. In other words, the dissertation considered individual differences in terms of preferences for each job characteristic directly contributing to strain and well-being 
outcomes. Also, using polynomial regression with higher order terms provides opportunity to examine curvilinear effects. And finally, examination of core job characteristics through the lens of PE fit for emergency services industry answers calls to further develop PE research in different organizational contexts (Edwards et al., 1998).

\section{Dissertation Outcomes of Interest: Strain and Well-Being}

As previously introduced, strain refers to psychological, behavioral, or physiological responses employees experience as a result of perceived stress from the work environment (see Ganster \& Rosen, 2013; Griffin \& Clarke, 2011). Organizational consequences associated with employee strain include increased medical costs, employee withdrawal (absenteeism, turnover), and decreased performance (Cooper, Liukkonen, \& Cartwright, 1996). Research with emergency responders has found a prevalence of strain outcomes, including burnout (Grigsby \& McKnew, 1988; Hooper, Craig, Janvrin, Wetsel, \& Reimels, 2010; Maslach, 1982), somatic symptoms, and generalized stress (e.g., Garbarino \& Magnavita, 2015; Gershon, Barocas, Canton, Li, \& Vlahov, 2009). These outcomes have been of specific interest in roles that deal with people in emotionally critical situations (e.g. psychotherapists, social workers, disaster response; Figley, 1999; Shakespeare-Finch, Wehr, Kaiplinger, \& Daley, 2014; Stamm, 2010). Therefore, outcomes selected for the dissertation were physical symptoms, burnout, and secondary traumatic stress.

Research aimed to integrate positive aspects of health and well-being in their own right have begun. Inclusion of positive dimensions of employee affect, cognition, and behavior during appraisal and in response to stressors may provide empirical support for positive resources for coping, active mental health, and increased employee engagement 
(Griffin \& Clarke, 2011). As previously introduced, indicators of affective and cognitive well-being have been used in organizational stress research. These indicators have included affective well-being (Bruk-Lee \& Spector, 2006), satisfaction (De Jonge \& Schaufeli, 1998; Warr, 1990), and positive organizational behavior (Bakker \& Schaufeli, 2008). Most research approaches have implicitly assumed that people experience strain at the expense of more positive outcomes such as positive affect or psychological morale (Hart \& Cooper, 2001). This assumption has unfortunately supported the idea of an occupational continuum in which strain and well-being are at opposite ends. This assumption is seen in studies with well-being operationalized as the absence of strain (Hart \& Cooper, 2001) or health as the absence of ill health (Warr, 2005). For the purposes of the dissertation, inclusion of compassion satisfaction as an indicator of wellbeing answered calls to examine this positive aspect in emergency services roles (Griffin \& Clarke, 2011; Hart \& Cooper, 2001).

Next, a brief review of literature regarding these strain and well-being outcomes is presented. This is followed by a more in-depth review of research that has examined relationships specifically between job characteristics and these outcomes.

\section{Physical symptoms.}

Physical symptoms (also referred to as somatization, psychosomatic symptoms) describe the presence of medically unexplained symptoms that imply a psychological component to the symptoms (Brown, 2004). Examples include headaches/migraines, indigestion/ulcers, and insomnia. As an unconscious process by which psychological distress is expressed, persistent somatization is associated with increased rates of disability and health care utilization (Schat et al., 2005). Physical symptoms are thought 
to be indicators of underlying mental health issues (such as anxiety, depression, and posttraumatic stress; Violanti et al., 2007) or indicators of potential onset of serious physical health concerns ( such as metabolic syndrome; Garbarino \& Magnavita, 2015). Prolonged or chronic exposure to work-related stressors have been associated with physiological effects that potentially lead to mental and physical illness (U.S. Department of Health and Human Services, National Institute for Occupational Safety and Health, 1999).

\section{Burnout.}

Job related burnout as a psychological strain outcome is characterized by extreme fatigue and loss of idealism and passion for one's job due to prolonged exposure to workplace stressors. Burnout has been conceptualized by various sub facets during its construct development and use in research. Maslach and colleagues (1982, 2003; Maslach \& Jackson, 1981) identified three dimensions of burnout as emotional exhaustion, cynicism (also called depersonalization), and feelings of inefficacy (also called personal accomplishment). Emotional exhaustion reflects an emotional overextension characterized by loss of feeling concerned or interested in one's job. Cynicism or depersonalization reflects one's response to the work and/or people associated with work (e.g., clients, coworkers) characterized by a callous or excessively detached response to other people or aspects of the job. Feelings of inefficacy or reduced personal accomplishment reflects one's negative response to themselves and their personal accomplishments. Feelings of inefficacy or reduced personal accomplishment reflects one's negative response to themselves. This is characterized by a lack in feeling competent or successful in one's work. 
Burnout in context of emergency services personnel has been conceptualized as feelings of hopelessness in doing an effective job and/or that their efforts make no difference (Stamm, 2010). This negative effect of helping others has been found prevalent in high-stress emergency responder roles. For example, paramedics and emergency room nurses had significantly higher average burnout scores compared to average burnout scores for other health-care positions (Grigsby \& McKnew, 1988). Hooper et al. (2010) also found emergency room nurses had moderate-to-high levels for burnout and compassion fatigue ( $82 \%$ and $86 \%$ respectively).

Environmental and personal factors were considered separately in Potter's (2006) review of literature investigating burnout among emergency room nurses and physicians. Environmental factors related to burnout included occupational hazards inherent to prolonged exposure to critical incidents, but also included organizational stressors such as long work hours, inadequate staffing, and lack of control. Emergency department nurses were typically found to have the least control and highest levels of burnout compared to non-emergency counterparts (Potter, 2006).

\section{Secondary traumatic stress.}

Secondary traumatic stress (STS) refers to "feelings of being trapped, on edge, exhausted, overwhelmed and infected by others' trauma" (Stamm, 2010, p. 21) resulting from work related exposure. STS is a psychological strain response that can occur for those who work with and help traumatized individuals and has been recently included as a subset of post-traumatic stress disorder according to the Diagnostic and Statistical Manual of Mental Disorders (American Psychiatric Association, 2013). People working in emergency services in various capacities often encounter traumatic situations, through 
primary and secondary exposures. Primary exposure to trauma refers to first-person experience of traumatic events. Examples include direct exposure to death, threatened death, actual or threatened serious injury, or actual or threatened sexual violence (American Psychiatric Association, 2013). For emergency responders, involvement in drug-busts, entering a house engulfed in flames, or being attacked by a potential suspect, would all be considered primary exposures. Experiencing repeated or extreme exposure to aversive details of such events, as is often the case for emergency responders, is also considered traumatic exposure (American Psychiatric Association, 2013). Sources of secondary traumatic exposure include gathering details of horrific injuries or scenes of recent serious injury, chronic exposure to intense or hysterical phone calls requesting emergency help, and dealing with the death of a coworker. STS results from helping or wanting to help a traumatized or suffering person and is not dependent on duration of exposure (Figley, 1999). In other words, STS can be experienced after one exposure or over a prolonged period of time.

While most research concerning the effects of STS have been conducted regarding therapists, social workers, and other health care roles, recent work has begun to examine STS in emergency responders (Burns, Morley, Bradshaw, \& Domene, 2008; Setti \& Argentero, 2014). Setti and Argentero (2014) examined the role of mindfulness and engagement as protective factors against STS and physical symptoms for firefighters. While they identified significant associations between STS factors and physical symptoms, results were inconclusive because of restricted variability in the strain outcome. In a qualitative study, Burns and colleagues (2008) examined protective factors for police investigating traumatic situations involving child exploitation. Burns et al. 
(2008) identified several factors that mitigated secondary trauma effects, including being the "right person for the job" (p. 26), proper training and prior experience, support from family, department, and community, and a sense of control over one's work.

Additionally, STS was found positively related to occupational tenure, such that higher levels of STS were present for those who had been in emergency services longer (Shakespeare-Finch, 2014). This suggested a higher likelihood of exposure to trauma due to longevity in emergency services.

\section{Compassion satisfaction.}

Those employed in emergency services may also experience a benefit in career satisfaction, resulting from a feeling of fulfillment in being part of a work that helps others (Stamm, 2002). Compassion satisfaction represents a positive, emotional response towards helping others. This aspect reflects one's pleasure in being able to effectively handle the challenges of emergency services, as well as in helping others through one's work. Compassion satisfaction is typically associated with positivity towards one's colleagues and workgroup, and with one's feelings of personal ability to contribute to the work setting or towards the greater good of society (Stamm, 2010).

Previous research using these outcomes included Trachik and colleagues' (2015) study that examined factors influencing burnout, STS, and compassion satisfaction for emergency dispatchers. Emergency dispatchers reported rates of burnout and STS significantly higher than normed population rates, which were significantly correlated to acute stress disorder symptoms. However, emergency dispatchers with higher compassion satisfaction levels were less likely to be diagnosed with acute stress disorder. Burnout and STS were significantly higher for dispatchers responsible for multiple 
services (e.g. fire, police, and medical), but not significantly related to tenure or shift work differences. Trachik and colleagues' (2015) work was instrumental by inclusion of positive outcomes of emergency services work in the outcome of compassion satisfaction. Overall, compassion satisfaction provides an opportunity to examine wellbeing outcomes for emergency responders amidst the challenges and negative effects of this high-stress industry.

\section{Relationships between Job Characteristics with Strain and Well-Being Outcomes \\ Operationalizations of job characteristics.}

As previously discussed, JCT posited that enriched and/or complex jobs contribute to positive organizational and individual outcomes (Hackman \& Oldham, 1976, 1980). At times, researchers have evaluated outcomes of job characteristics by creating a single index or composite of several dimensions to reflect "the overall potential of the job environment to foster motivation and satisfaction on the part of job holders" (Kulik et al., 1987, p. 282). These composite measures have been called job scope (Xie \& Johns, 1995), job enrichment (Fried et al., 2013), and job complexity (Bowling \& Hammond, 2008) to name a few. Bowling and Hammond's (2008) meta-analysis included a composite of overall job complexity and found a moderately strong relationship to job satisfaction $(\hat{\rho}=.46$; Bowling \& Hammond, 2008). Similarly, job characteristics as a composite score has been found related to job satisfaction (Champoux, 1980), exhaustion (Xie \& Johns, 1995), and physical health outcomes (Fried et al., 2013).

In fact, Champoux's (1980) study was one of the first to identify curvilinear effects from a summed composite of all five JCT core job characteristics. Specifically, a 
moderate job scope related to high levels of job satisfaction and motivation, whereas lower and higher levels of job scope were associated with decreases in satisfaction and motivation (Champoux, 1980). Likewise, in both Xie and Johns' (1995) and Fried and colleagues' (2013) research, curvilinear effects were found between a job characteristics composite and well-being outcomes. For Xie and Johns' (1995) study, higher burnout levels were found when job scope was either low or high; whereas Fried et al. (2013) found negative health outcomes (measured by obesity indicators) increased when job enrichment exceeded average. These findings are consistent with activation theory that states every task has an optimum level associated with maximized benefits (i.e., reduced strain, increased well-being) and that below or beyond this optimum level, positive outcomes decline (Gardner, 1986; Scott, 1966; Warr, 1987). Requests for researchers to examine these curvilinear effects between job characteristics and employee outcomes have been made (Humphrey, Nahrgang, \& Morgeson, 2007) and answered in recent years (Fried et al., 2013; Huyghebaert et al., 2016).

However, findings have not been consistent regarding the relationship between job characteristics to strain and well-being outcomes. In meta-analysis conducted by Humphrey et al. (2007), job complexity was found related to job satisfaction $(\hat{\rho}=.37)$ but unrelated to generalized stress and burnout. This relationship was not supported in subsequent research conducted by Chung-Yan (2010) in which job complexity, measured by the same operationalization and scale, was not related to job satisfaction nor psychological well-being.

Across several theoretical approaches, relationships between job characteristics and strain and well-being outcomes have been of interest for decades (see Bliese et al., 
2017). Job characteristics dimensions specific to this dissertation have typically found outcomes related to generalized stress, anxiety, and burnout (Griffin \& Clarke, 2011; Kahn \& Boysiere, 1992). The remainder of this section emphasizes research that has examined the relationships of job characteristics to strain and well-being outcomes of interest in this dissertation, with a special focus on emergency services personnel.

\section{Between skill variety and strain or well-being.}

The job characteristic of skill variety refers to a job that requires use of a variety of skills for successful completion of the work (Hackman \& Oldham, 1976, 1980). Related terms used in research have been job variety (i.e., the level of variation in the job allowing for different experiences and tasks; Griffin et al., 2012) and task variety (i.e., a job requiring employees to perform a wide range of tasks; Morgeson \& Humphrey, 2006). Generally, meta-analytic results have found skill variety significantly related to satisfaction $(\hat{\rho}=.28, .42$; Bowling \& Hammond, 2008; Humphrey et al., 2007; respectively). This is consistent with the expectation that enriched jobs that include a wide range of tasks, skills, or activities are more personally meaningful for employees (Hackman \& Oldham, 1976, 1980) contributing to positive well-being indicated by satisfaction.

However, relationships between skill variety and strain outcomes have been inconsistent. For example, while Xie and Johns' (1995) study found skill variety related to exhaustion in a multiple industries sample, this significant relationship was not found in meta-analyses conducted by Humphrey et al. (2007) in which no significant relationships between skill variety and burnout, nor between skill variety and generalized job stress were identified. Humphrey and colleagues' (2007) non-significant finding was 
consistent with subsequent research conducted by Zaniboni, Truxillo, and Fraccaroli (2013) in which task variety $(\beta=0.02, n s)$ and skill variety $(\beta=0.13, n s)$, as measured by Morgeson and Humphrey's (2006) Work Diagnostic Questionnaire (WDQ), were not predictive of work-related burnout for clerical and managerial roles.

\section{Skill variety relationships in emergency services occupations.}

Griffin and colleagues' (2012) study with corrections staff found job variety significantly related to three burnout facets: emotional exhaustion $(r=-.41)$, depersonalization $(r=-.44)$, and feelings of ineffectiveness when dealing with others ( $r=$ -.39). Griffin and colleagues (2012) suggested that the presence of job variety allowed corrections staff to address job-related issues in creative ways, thereby reducing the frustration associated with burnout. In contrast, this was not the case in Lambert and colleagues' studies (Lambert, 2004; Lambert, Hogan, Dial, Jiang, \& Khondaker, 2012) that found job variety significantly predictive of job satisfaction $(\beta=0.29, p<.01)$, but not a significant predictor of burnout $(\beta=0.05, n s)$ for correction officers.

This inconsistency in relationships suggests possible curvilinear relationships between skill variety and outcomes. According to the activation theory (Scott, 1966), job stress may occur when job-related stimuli cause an employee's experienced activation level to deviate significantly from their customary level (Gardner, 1986; Scott, 1966). In other words, skill variety may potentially result in strain through under- or overstimulation (Hackman \& Oldham, 1976, 1980; Scott, 1966) and present a barrier to one's obtainment of need satisfaction. Such inconsistencies in findings may be identified by examination of the nature of person-job fit and misfit in the dimension of skill variety. 


\section{Between task identity and strain or well-being.}

In JCT, Hackman and Oldham $(1976,1980)$ have suggested that jobs that involve a whole piece of work are more interesting and rewarding to employees than jobs that involve only small parts of the task. The relationship between task identity and psychological outcomes has been generally supported in meta-analyses. Specifically, task identity has shown moderate relationships with satisfaction $(\hat{\rho}=.28, .31$; Bowling $\&$ Hammond, 2008; Humphrey et al., 2007; respectively). These meta-analytic findings were consistent with Morgeson and Humphrey's (2006) multiple industries study, in which task identity was significantly related to job satisfaction $(r=.13)$.

Task identity has also been consistent in its relationship with strain outcomes. For example, it was significantly related to exhaustion $(\hat{\rho}=-.28)$ and generalized job stress ( $\hat{\rho}=-.17$ ) in Humphrey et al.'s (2007) meta-analysis. Additionally, in Xie and Johns' (1995) multiple industries research, task identity was not only significantly related to exhaustion ( $r=-.16)$ but was a primary contributor to the curvilinear effects between job scope and burnout.

\section{Task identity relationships in emergency services occupations.}

Research examining the relationship between task identity and strain and wellbeing outcomes for emergency responders was more limited. A notable exception was research conducted by Huyghebaert and colleagues (2016) with a nursing professionals sample. Their findings suggested a curvilinear relationship between task identity and nurses' need for recovery. Need for recovery as a strain outcome is characterized by lack of energy, reduced performance, and feelings of overload (Huyghebaert et al., 2016). As the perceived presence of task identity increased to a moderate level, nurses' need for 
recovery decreased. However, after the mid-point in perceived task identity was surpassed, need for recovery increased. This suggested the job characteristic of task identity to have diminishing returns in terms of influence on strain. In other words, efforts to increase employees' experience with their work from beginning-to-end past a moderate level had lessened beneficial effects for nurses in terms of their need for recovery. An oft-mentioned aspect of caring for others has included the feeling of being overwhelmed by how significantly victims' or clients' needs are dependent upon a careworker's performance (Figley, 1999; Maslach, 1982; Maslach \& Jackson, 1981; Stamm, 2002). This excess of being too closely identified with the task might contribute to strain due to over-commitment, or over-immersion in emergency services.

\section{Between task significance and strain or well-being.}

Task significance refers to the impact a job or task has on the lives and well-being of others (Hackman \& Oldham, 1976, 1980). Employees with jobs that contribute to the physical or psychological well-being of others are considered more likely to experience greater meaningfulness in their work. The range of meta-analytic relationships reported for task significance with job satisfaction has been fairly wide, from $\hat{\rho}=.17$ (Bowling \& Hammond, 2008) to $\hat{\rho}=.41$ (Humphrey et al., 2007). In Morgeson and Humphrey's (2006) research, task significance was moderately related to job satisfaction $(r=.33)$. In contrast, relationships between task significance and exhaustion have been relatively consistent, such that meta-analytic estimate $\hat{\rho}=-.29$ (Humphrey et al., 2007) was close to Xie and Johns' (1995) multiple industries correlation $r=-.15$. Of note, in Xie and Johns' (1995) study, the curvilinear effect found between a composite of all job characteristics (i.e., job scope) and burnout was primarily due to curvilinearity of task 
significance and task identity characteristics. Specifically, levels of burnout were reduced as task significance increased towards the midpoint of responses. Once perceived presence of task significance exceeded the midpoint, burnout increased (Xie \& Johns, 1995).

\section{Task significance relationships in emergency services occupations.}

In terms of emergency responder roles, Grant (2008) examined how workplace initiatives to increase task significance might change employee attitudes of job dedication, and increase helping behavior and feelings of social contribution for lifeguards. He found that those in a task significance intervention condition were rated higher in these outcomes (Grant, 2008). While limited work has been reported regarding the relationship between task significance and strain or well-being outcomes for emergency responders, findings have suggested a curvilinear relationship might help to explain the range of meta-analytic relationships previously reported.

\section{Between autonomy and strain or well-being.}

\section{Operationalizations of autonomy.}

In contrast to the limited consideration given to task significance, autonomy has been the favored of job characteristics dimensions and has enjoyed the longest theoretical and empirical attention. In the workplace, autonomy has predominantly been studied in context of job redesign efforts and operationalized in various ways. Consistent with JCT, autonomy was initially viewed as the amount of freedom and independence an individual has in terms of carrying out his or her work assignment (Hackman \& Oldham, 1976, 1980). Job-Demands-Control (JDC) and its extension Job-Demands-Control Support models (Karasek, 1979; Karasek \& Theorell, 1990) have examined autonomy in the 
workplace as job control with two facets, skill discretion and decision authority, which denote the extent of autonomy an employee has in deciding how to meet job demands. Job Demands Resources model (JDR; Bakker \& Demerouti, 2007) designated autonomy as a one of many resources employees might draw upon to meet various job demands. And finally, in Morgeson and Humphrey's (2006) research, autonomy was conceptualized in three interrelated aspects denoting freedom in work scheduling, decision making, and work methods.

Consistent across these operationalizations is the notion that jobs characterized by autonomous decision making contribute to employee motivation and positive outcomes (Hackman \& Oldham, 1976, 1980). Freedom in how and when to perform work duties facilitates employee's attainment of intrinsic needs (Ryan \& Deci, 2000) such as growth, learning and development (Hackman \& Lawler, 1971), competence (White, 1959), and relatedness (Baumeister \& Leary, 1995), as well as extrinsic motivational needs such as achieving work goals (Bakker \& Demerouti, 2007).

Several meta-analyses have confirmed moderate-to-strong relationships between autonomy and strain or well-being outcomes. Specifically, meta-analysis conducted by Bowling and Hammond (2008) found autonomy related to job satisfaction $(\hat{\rho}=.35)$; Humphrey et al.'s (2007) meta-analysis found autonomy related to job satisfaction $(\hat{\rho}=$ $.48)$, exhaustion $(\hat{\rho}=-.30)$ and generalized job stress $(\hat{\rho}=-.23)$; and Spector's (1986) meta-analysis found autonomy significantly related to physical symptoms $(\hat{\rho}=-0.33)$ and emotional distress $(\hat{\rho}=-0.37)$. More recently, meta-analysis conducted by Fila, Purl, and Griffeth (2017) confirmed relationships between autonomy with satisfaction $(\hat{\rho}=0.46)$ and between autonomy with exhaustion $(\hat{\rho}=-0.20)$. 
Recent studies using a variety of work industries included Chung-Yan's (2010) examination of job complexity and job autonomy. Chung-Yan (2010) used Morgeson and Humphrey's (2006) model of work design that included categories of motivational, social, and contextual aspects of work. Autonomy subscales were combined into one score and found related to job satisfaction $(r=.39)$, turnover $(r=-.22)$, and psychological well-being $(r=.33)$. Likewise, Xie and Johns' (1995) study found a significant relationship between autonomy and exhaustion $(r=-.28)$. Of note, while both ChungYan (2010) and Xie and Johns' (1995) studies examined curvilinear effects, neither study identified significant curvilinear main effects from job autonomy on strain or well-being outcomes. However, Chung-Yan (2010) found an interaction between job autonomy and curvilinear job complexity such that in cases of high autonomy, high job complexity was related to increased psychological well-being $(\beta=-0.31, p<.01)$. This finding speaks to the complex nature of job characteristics' relationship with well-being (Chung-Yan, 2010).

\section{Autonomy relationships in emergency services occupations.}

While the JDC framework has received some criticism regarding its explanation of interactive effects between job characteristics (Häusser, Mojzisch, Niesel, \& SchulzHardt, 2010), JDC has been a predominant model used to identify strain or well-being outcomes for healthcare populations (see reviews by Adriaenssens, De Gucht, \& Maes, 2015; Häusser et al., 2010). Recent research using a nurse population confirmed relationships between autonomy facets and outcomes of job satisfaction and burnout facets (Pisanti, van der Doef, Maes, Lazzari, \& Bertini, 2011). Specifically, skill discretion and decision authority evidenced a moderate relationship with job satisfaction 
$(r=.39, .33$; respectively); whereas a more modest relationship was identified for burnout facets of emotional exhaustion $(r=-.18,-.21)$, depersonalization $(r=-.12,-.13)$, and personal accomplishment $(r=.17, .21$; Pisanti et al., 2011). This pattern of autonomy having a stronger relationship with job satisfaction compared to a small or non-significant relationship to burnout has been consistent for healthcare roles (see Adriaenssens et al., 2015; Rouxel, Michinov, \& Dodeler, 2016).

In Gelsema et al. (2006), a two-wave study identified relationships between a change in autonomy-based work conditions and nurses' health and well-being outcomes. With a three-year interval between assessments, changes in skill discretion $(\beta=0.18, p<$ $.001)$ and decision authority $(\beta=0.19, p<.001)$ were predictive of job satisfaction. In contrast, these changes in autonomy were not significant in the prediction of exhaustion $(\beta=0.03, n s$ and $\beta=-0.15, n s ;$ respectively).

Research concerning health and well-being outcomes for other emergency responder populations have often included the job characteristic dimension of autonomy. For example, Martinussen, Richardsen, and Burke (2007) examined relationships between job demands, job resources, and burnout for police in a nationwide health care initiative in Norway. Their findings indicated significant relationships between job autonomy and three components of burnout: exhaustion $(r=-0.20)$, cynicism $(r=-0.14)$, and personal efficacy ( $r=0.15$; Martinussen et al., 2007). This was consistent with findings that police officers were more likely to experience psychological strain in workplaces that lacked autonomy (Collins \& Gibbs, 2003). Likewise, Lambert and colleagues (Lambert, 2004; Lambert et al., 2012) found job autonomy as a significant 
predictor of increased job satisfaction $(\beta=0.20, p<.05)$ and of reduced burnout $(\beta=$ $0.32, p<.01)$ for prison correction officers.

Autonomy is exceptionally amendable to job enrichment initiatives such that even minor opportunities in decision latitude can help employees connect with their work, feel that their input is valued, and increase commitment to the work and organization. As such, findings from this dissertation may be immediately useful to organizational change initiatives. Even with autonomy's prominence in research, some questions remain about its curvilinear effects and how one's preferences change the relationship between provided work autonomy and outcomes. Evaluation of autonomy fit aimed to identify these nuances of fit and misfit in relation to employee strain and well-being.

\section{Between job-based feedback and strain or well-being.}

As posited in JCT, knowledge of the effectiveness of one's performance facilitates higher order needs satisfaction (Hackman \& Oldham, 1976, 1980). However, research examining feedback as a job characteristic does not always differentiate between agent-based feedback (e.g., from supervisor, coworkers, clients) and job-based feedback

(i.e., from the job itself). Job-based feedback reflects the degree a job provides direct and clear information about one's effectiveness at work. This focus on job-based feedback is expected to provide employees with knowledge of the results of their performance in a timely manner and thereby contribute to individual outcomes (Hackman \& Oldham, 1976, 1980).

Meta-analyses have consistently found moderately strong relationships between job-based feedback and job satisfaction $(\hat{\rho}=.46, .43$; Bowling \& Hammond, 2008; Humphrey et al., 2007; respectively). This was in contrast to smaller relationships found 
in studies conducted by Morgeson and Humphrey (2006) which used a multiple industries sample $(r=.22)$ and Sekaran's (1989) research with bank employees $(r=.35)$.

Job-based feedback relationship with the strain outcome of generalized job stress was reported at $\hat{\rho}=-.21$ in Humphrey and colleagues' (2007) meta-analysis; whereas a stronger $(r=-.35)$ correlational relationship between job-based feedback and job stress was reported by Sekaran (1989). More specific to the outcome variables of interest in this dissertation, Xie and Johns' (1995) reported a significant relationship between jobbased feedback and the burnout facet of emotional exhaustion $(r=-.21)$.

\section{Feedback relationships in emergency services occupations.}

Research highlighting these relationships for emergency responders included work conducted by Lambert and colleagues (2012) for prison staff in which feedback (combined agent- and job-based) predicted reduced burnout $(\beta=-0.15, p<.05)$. In earlier work, Greller and Parsons (1992) examined the role of feedback for metropolitan police officers. Specifically, they considered differences between job-based and supervisor's feedback and found job-based feedback had a stronger association with officer's self-rating of performance, whereas supervisor's feedback was more strongly related to strain (Greller \& Parsons, 1992).

As such, research in which the relationship between job-based feedback and strain or well-being outcomes were examined was limited. Yet, based on these related examples, excess in job-based feedback past one's preference for feedback may produce overstimulation and overload for employees in emergency services. This expectation is consistent with findings in which overstimulation was related to emotional numbness for emergency dispatchers (Shuler, 2001) and findings that prolonged and repeated exposure 
to traumatic events have resulted in symptoms of STS and emotional desensitization (Figley, 1999).

\section{Summary of relationships between study variables.}

In summary, meta-analyses have confirmed relationships between job characteristics dimensions with job satisfaction, burnout, and generalized stress (Bowling \& Hammond, 2008; Humphrey et al., 2007). Relationships between job characteristics and outcomes have typically been stronger for psychological outcomes like satisfaction than with burnout or somatic symptoms, which is consistent with foundational PE fit research (Harrison, 1978).

Consistent throughout previous research that has examined relationships between job characteristics and strain or well-being has been the assumption that more is better, regardless of personal preferences. In other words, higher levels of job complexity, enrichment, autonomy, feedback, and so on were expected to reduce strain and increase well-being. Inconsistent results (e.g., Gelsema et al., 2006; Lambert et al., 2012) have suggested this assumption might not always be the case. Also, curvilinear effects found between job characteristics and key outcomes (e.g., Champoux, 1980; Chung-Yan, 2010;

Fried et al., 2013; Huyghebaert et al., 2016; Xie \& Johns, 1995) suggest the relationship between job characteristics and psychological health may be more complex than previous approaches have considered. This dissertation aimed to include one's preference for each

job characteristic in effort to better understand these outcomes from a PE fit perspective.

\section{Research Questions Addressed in the Dissertation}

The dissertation examined how the nature of fit and the nature of misfit, along JCT job characteristics dimensions, were related to strain and well-being outcomes for 
emergency responders. In summary, compatibility between one's preference for and perceived presence of job attributes of skill variety, task identity, task significance, autonomy, and job-based feedback were examined in relation to outcomes of physical symptoms, burnout, secondary traumatic stress, and compassion satisfaction. As such, two overarching research questions were examined for each fit-outcome relationship:

1) What is the relationship with strain and well-being outcomes when one's preference for and perceived presence of a job attribute are matched?

Corollary: Does a matched low preference/low presence of a job attribute have a different relationship to strain and well-being outcomes compared to a matched high preference/high presence of the same job attribute?

2) When there is a discrepancy between one's preference for and perceived presence of a job attribute, does this discrepancy relate to strain and wellbeing outcomes?

Corollary: When there is a discrepancy between what employees desire in their job and their perception of the organization's supply, should the organization be more concerned with oversupply or undersupply of employees' preferences?

A polynomial regression with response surface analysis approach was used to answer these questions. Detailed discussion of how the response surface is created, tested, and evaluated is provided in Chapter III: Method, Data Analysis Plan. 


\section{CHAPTER III: METHOD}

\section{Participants}

\section{Sample profile.}

The targeted population for the dissertation concerned those employed in emergency services. In the most general sense, an emergency responder is someone designated or trained to respond to an emergency. According to the Bureau of Labor Statistics, Occupational Outlook Handbook (U.S. Department of Labor, Bureau of Labor Statistics, 2015), roles typical of emergency response such as police officers, detectives, security guards, and firefighters are classified within Protective Services occupations. Other notable roles often called upon in emergencies were classified elsewhere, such as police/fire/medical dispatchers in Office and Administrative Support, air traffic controllers in Transportation and Material Moving, and emergency medical technicians (EMTs) and paramedics in Healthcare. For the purposes of the dissertation, emergency responders of interest included those regularly exposed to primary and/or secondary trauma in their response to emergency situations. Targeted occupations included police officers, firefighters, police/fire/medical dispatch, EMTs and paramedics.

Table A2 (in Appendix A) summarizes the occupational outlook for these targeted emergency responder roles. Of note, total workforce for emergency responders is less than $1 \%$ of the estimated population. Also, EMTs and paramedics are the least compensated while having the highest projected growth rate. Review of the Occupational Information Network (O*NET; Peterson et al., 2001) job analysis database was conducted to assess requirements and expectations inherent to emergency responder roles. Specifically, areas reviewed included the generalized work style, task activity, and 
work context domains for emergency responder positions of interest. These domains in $\mathrm{O} * \mathrm{NET}$ reflect the occupational requirements directly related to what is done in a job and the surrounding context of that job. Across targeted emergency responder occupations, the following similarities were noted:

- Work style expectations were the same across all emergency responder occupations, including areas of stress tolerance, persistence, adaptability/flexibility, initiative, concern for others, and cooperation.

- Task expectations were similar across emergency responder occupations such that each had duties in maintaining equipment, information, and personal readiness for an emergency, coordinating between agencies in response to emergencies, responding within their respective specialization to emergency scenes, and follow-up administrative duties of documentation or legal procedures. Police and firefighters also had duties related to public awareness or education.

- Aspects of the work context deemed fundamental across emergency responder occupations included the serious consequences of error, constant contact with others, dealing with angry/unpleasant people, frequency and impact of decisionmaking, the importance of being accurate in communications and report, and the importance of teamwork.

Purported advantages of working in emergency services include the pride or satisfaction of making a difference in someone's life, competitive salaries (e.g. \$46k\$60k for fire and police), average employment growth, and ease of getting started in emergency services with high school diploma and agency training or certification; 
disadvantages included the likelihood of working in dangerous environments, irregular or long work hours, and the physical and emotional stress inherent in these positions (U.S. Department of Labor, Bureau of Labor Statistics, 2015). Because of the similarities in work environment, task expectations and work style, the current study examined impact of PE fit upon well-being and strain outcomes across all emergency responder roles.

\section{Participants (in analysis).}

Final dataset included 358 participants, with a distribution of male $(n=212$, $59.2 \%)$, female $(n=71,19.8 \%)$, and respondents who did not indicate gender $(n=75$, 20.9\%). Participants reported an average age of 44.79 years $(S D=9.27)$ and indicated their ethnic backgrounds to include Caucasian $(n=228,63.7 \%)$, Hispanic $(n=29,8.1 \%)$, African-American $(n=9,2.5 \%)$, and Other $(n=14,4.0 \%)$, with $78(21.8 \%)$ respondents not reporting ethnicity.

Education background levels of participants was distributed across high school ( $n$ $=18,5.0 \%)$, some college $(n=138,38.5 \%)$, Bachelors $(n=75,20.9 \%)$, and advanced degrees $(n=52,14.5 \%)$. Income levels ranged from $\$ 20,000$ to over $\$ 100,000$, with most participants earning over $\$ 75,000(n=217,60.6 \%)$.

Represented branches of emergency services included Law Enforcement/Police ( $n$ $=162,45.3 \%)$, Firefighters $(n=64,17.9 \%)$, Emergency Dispatchers $(n=32,8.9 \%)$, EMT/Paramedics $(n=6,1.7 \%)$, and Other $(n=21,5.9 \%)$, with $73(20.4 \%)$ participants not reporting their branch of emergency services. Of note, several participants indicated dual branch service, either concurrently (e.g., fire-rescue) or sequentially (e.g., initially in law enforcement, then changed to firefighter). In these cases, participants were represented in their primary or current position, respectively. Organizational $(M=14.75$ 
years, $S D=8.57$ years $)$ and occupational $(M=18.94$ years, $S D=9.19$ years $)$ tenure ranged from a few months to 39 and 43 years respectively.

\section{Procedure}

\section{Participant recruitment.}

Because a wide range of emergency services personnel were sought for inclusion, a broad spread recruitment to local, state and national emergency responder associations was implemented. Of those associations in which a primary contact was obtained, opportunities to personally introduce the study and recruit from the local department were utilized. Recruitment materials consisted of a one-page flyer, a one-page email, and business cards that provided study information. These materials were made available to organizations and associations related to police, firefighters, EMTs/paramedics, and emergency dispatchers.

\section{Survey distribution.}

The measures described below were combined into one survey and distributed online using Qualtrics Online Survey Software. Upon following the provided URL link, participants were provided the informed consent with details about the study. Informed consent was approved by the IRB and included study purpose and approximate duration of the survey, disclosure of risks and benefits associated with participation, notification to participant regarding their right of withdrawal from the study, and assured confidentiality of any personally identifiable information collected. Contact information was included for both the IRB office at Florida International University and the primary investigator.

Upon acceptance of the informed consent, participants received an informational dialog to orient them to the online survey environment. Details, such as buttons used to 
forward progress through the survey and the progress bar to indicate how much of the survey was completed, were pointed out along with an appreciation statement regarding their time and commitment to emergency services work. The survey consisted of previously validated scales to assess workplace stressors and strains or well-being outcomes of interest as described below, followed by items to assess demographic characteristics. All scales and questions are provided in Appendix B.

\section{Compensation.}

At the start of data collection, funds were not available for participant compensation or incentives. Therefore, participants were informed that no payment would be forthcoming. However, because of the potential for future funding, participants were given the option to include contact information to receive funds should they become available in the future. Once funding was acquired, the first 200 respondents who had indicated their desire to be included in potential incentives and provided contact information were sent an Amazon e-gift card (\$10) via email. Of note, public service agencies' policies regulating acceptance of gifts from the public may have required some respondents to decline this offer of compensation.

\section{Measures}

\section{PE fit components.}

Sections 1 and 2 of the Job Diagnostic Survey (JDS) created by Hackman and Oldham (1980), were modified to reflect measures of preferred and supplied job characteristics in five content dimensions of skill variety, task identity, task significance, autonomy, and job-based feedback. These dimensions were chosen based on their relevance to the sample and their prevalence in work motivation research. Six items were 
used for each job dimension, yielding 30 items total for the five job dimensions. As developed by Hackman and Oldham (1980), one item used a 7-step rating format with short descriptive phrases anchoring its middle and end points. The remaining items used a 7-point Likert response ranging from 1 (strongly agree) to 7 (strongly disagree). As an example, "I prefer a simple and repetitive nature of the work, without a lot of complexity." (reverse scored) represented the person component of the content dimension of skill variety; whereas, "My job is basically simple and repetitive.” (reverse scored) represented the environment component of skill variety. From the Qualtrics data collection, missing item-level responses ranged from $166(25.4 \%)$ to $258(39.4 \%)$ for JDS scales.

\section{Strain and well-being outcomes.}

The Physical Symptoms Inventory (PSI) developed by Spector and Jex (1998) was used to assess symptomatic outcomes experienced by emergency services personnel. This 12-item scale asked respondents to report frequency of various psychosomatic symptoms such as stomach upset/indigestion/cramps, constipation/diarrhea, dizziness/fatigue, sleeping issues, eye/ear strain, and headaches. Each item began with the stem phrase, "During the past 30 days, did you have ...?", response options ranged from 1 (not at all) to 5 (every day). Response values were summed to a total score and ranged from 12 to 60 with higher scores indicating more frequent somatic symptoms.

The Professional Quality of Life (ProQOL) questionnaire was developed by Stamm (2010) to assess areas of compassion satisfaction and compassion fatigue specifically for care-giving personnel and used a 30-item scale with a 5-point Likert response of frequency $(1=$ never to $5=$ very often $)$. Compassion satisfaction was 
measured with 10 items, an example being "My work makes me feel satisfied." Compassion fatigue included sub facets of burnout and secondary traumatic stress using 10 items each. An example item was, "I feel trapped by my job as an emergency responder." Subscales were computed by mean, which provided a range from 1 to 5 for each subscale. Reported reliability for ProQOL facets: compassion satisfaction $(\alpha=.88)$, burnout $(\alpha=.75)$, and secondary traumatic stress $(\alpha=.81)$.

From Qualtrics data collection, outcome variables reported almost half of their cases with missing values. Specifically, PSI had missing item-level responses in 326 (49.8\%) cases and ProQOL was missing item-level responses in 297 (45.4\%) cases.

Demographics data were collected through 15 items that asked respondents to report occupational and organizational tenure, employment status, branch of service, age, ethnicity, gender, educational background, and income.

\section{Data Analysis Plan}

As discussed in the Literature Review, polynomial regression facilitates a more complete examination of the fit-outcome relationship than two-dimensional congruence via difference scores (Edwards, 2002). Ordinary Least Squares (OLS) method is commonly considered the best linear unbiased estimator as it minimizes sum of squares differences between observed and predicted outcome values according to the regression equation (Cohen et al., 2003). However, OLS abilities are hampered when data are missing and/or violations of assumptions of OLS are present. In these instances, multiple imputation (MI) or maximum likelihood (ML) are recommended. These techniques use all information in present data to inform and either impute (MI) or estimate (ML) regression coefficients and standard errors that best fit the sample. This section discusses 
techniques used for handing missing data, assumptions and requirements of regression analysis within the context of congruence research, and use of ML to estimate regression coefficients and response surface parameters.

\section{Mechanisms and levels of missing data.}

\section{Mechanisms of missingness.}

Missing data are "a statistical problem characterized by an incomplete data matrix that results when one or more individuals in a sampling frame do not respond to one or more survey items" (Newman, 2014, p. 373). Data can be missing at random having no relationship with the measured concepts themselves, missing systematically such that missing items are directly related to the concepts, or anywhere in between (Newman, 2014). Three mechanisms that describe this continuum of missingness include missing completely at random (MCAR), missing at random (MAR), and missing not at random (MNAR; Enders, 2010; Little \& Rubin, 1987; Newman, 2014; Schafer \& Graham, 2002).

Missing completely at random refers to the probability that missing outcome values do not depend on observed values, nor are they related to the missing data values themselves. For example, a power outage that results in lost cases on a researcher's computer, would not be related to any of the study's constructs.

Missing at random refers to missing values that may partly depend on other observed values, but not related to missing values themselves. An example of this would be if respondents failed to report income and this was related to their marital status. In other words, while marital status is not the missing variable itself, participants who are married tend to not report income as often as unmarried participants. This would 
represent MAR data, as missingness on the income variable was related to another observed variable (i.e., marital status).

Missing not at random is considered systematic missingness as missing data are directly related to the values missing themselves. Missing not at random might be seen in constructs related to sensitive issues - such as drug dependency or infidelity. For example, participant might not complete a drug intervention program (and therefore have missing values for drug use) because they had succumbed to their addictive behavior and were not available to take the survey. In this situation, missing values are directly related to the construct being measured.

In summary, mechanisms for missingness have to do with how data became missing, how missing values relate to the variable itself, and how missing values relate to other variables. While the presence of MCAR is not statistically possible, Little's test for MCAR evaluates mean differences across subgroups of cases with similar missing data patterns in the dataset, which may indicate data are not MCAR (Enders, 2010). A significant Little's MCAR test would suggest data are either MAR or MNAR, and thereby benefit from missing data techniques to estimate the model (Enders, 2010). As will be discussed in more detail later, missing data are problematic for model estimation as they introduce bias in the estimation of regression parameters and standard errors for significance testing.

\section{Levels of missingness.}

Missing data correspond to three levels of analysis: item-, construct- and personlevel missingness (Newman, 2014). 
Item-level missingness occurs when a few items are left blank on a multi-item scale (Newman, 2014). Techniques used to handle item-level missingness (when subsequent analyses are focused on construct-level models) include list-wise deletion of cases responding under a specified cutoff and using the mean across available items per respondent to calculate a construct-level value. While neither of these techniques for dealing with item-level missingness are unbiased for MAR data, some researchers have advocated use of person mean imputation when more than half of responses are available (i.e., a combination of list-wise deletion and mean of available items; Enders, 2010; Graham, 2009).

When all items within a scale are missing, this becomes construct-level missingness. Participants who have responded to some constructs, but not all constructs in a survey are called partial respondents (Newman, 2014). In other words, constructlevel missingness occurs when a participant answers zero items from a at least one scale in the survey. When data are MCAR or MAR, MI and ML techniques provide unbiased estimates of parameters and standard errors for construct-level missingness.

Construct-level missingness was addressed using a full information maximum likelihood (FIML) algorithm that directly analyses the incomplete dataset to yield unbiased parameter estimates and accurate standard errors. For this estimation technique, variables specific to the model (including interactions and higher order terms) as well as auxiliary variables (that are not of any substantive interest to the study) are included so that the algorithm has all available information to estimate parameters of best fit to the data (Newman, 2014). 
Failure to respond to any part of the survey would indicate person-level missingness as a nonrespondent. Person-level missingness is the most difficult to address because, with no observed variables specific to the participant, there are no missing data techniques that can yield unbiased parameter estimates (Newman, 2014). Therefore, nonrespondent participant cases were completely removed from analyses.

\section{Approach to missing data.}

As estimated or imputed values become less dependable as the proportion of missing data increases, several steps were taken to handle missingness in the dataset. To reduce problems with list-wise deletion and limit the number of estimated or imputed values needed, cases were retained when predictor variables were complete, and an outcome variable scale was at least $80 \%$ complete. This approach retained the most cases $(N=358)$ while allowing opportunities to use ML to account for missingness during model estimation.

Item level missingness for outcome scales was handled by computing scale scores by average for all cases with at least $80 \%$ response. This technique has been referred to as person mean imputation or prorated scale score (Enders, 2010). While not actually assigning values for item-level individual cases, use of person mean imputation to compute scale-level scores is equivalent to imputing the missing values with the mean of a participant's completed items. Some limitations in using prorated scale score technique have been identified when data are MCAR or MAR, and for estimating internal reliability (Enders, 2003; Schafer \& Graham, 2002).

Maximum likelihood was used to estimate values for parameters most likely to occur using all information available (Enders, 2010). For example, the current sample of 
emergency responders represented in the dataset is part of a larger emergency responder population. The likelihood of this sample's characteristics (i.e., sample mean, sample variance) being produced by the population is compared against several 'try-outs' of potential population parameters (i.e., population mean, population variance) to identify which estimates are most plausible. In other words, ML determines after 1,000 or even 10,000 repetitions of try-outs, which population estimates were most likely - relatively compared across all potential population estimates - to produce this sample's characteristics (Enders, 2010).

In estimation of regression coefficients, the same iterative process determines the most likely regression parameters using the sample data. Similar to the population estimates example, repetitions of try-outs for potential regression coefficients are compared relative to each other for their plausibility or likelihood of producing the sample characteristics. Despite missing values, ML is still able to quantify the joint probability of drawing the observed data from a normally distributed population. The goal of ML estimation is to identify specific patterns of estimates that produce the highest likelihood; and therefore, represent the best fit to the data. Of note, ML estimation does not to impute or create values for sample cases, but provides parameter estimates and standard errors for the model itself (Enders, 2010). Limitations noted for this technique have been reported for small sample sizes and MCAR or MAR data (Enders, 2003; Schafer \& Graham, 2002). As with regression, ML also depends on a multivariate normal distribution. The following section discusses assumptions required for polynomial regression and congruence research, as well as multivariate outliers detection. 


\section{Assumptions related to polynomial regression.}

Assumptions refer to the nature of data required for accurate model testing.

Parametric tests use probability calculations, and as such depend on a normal distribution of data, or at least data adhering to a known probability distribution. For example, tests of significance assume a predetermined risk level (e.g., $p<.05$ ). Specifically, a risk level set at $p<.05$ indicates the risk of making a Type I error, such that the probability of finding an effect in the sample not truly present in the represented population is less than $5 \%$. Depending on the type of model testing used (e.g., polynomial, ANOVA, correlation), certain assumptions are required for valid results.

Violations of assumptions in regression analysis creates two main issues in regard to accurate model estimation. First, they can bias estimates of regression coefficients. In other words, when violations of assumptions are present in the data, estimates of regression coefficients based on the sample may not accurately reflect the true values of those regression coefficients in the population. Second, violation of assumptions can bias the estimate of standard error. As an indicator of variability in the data, standard error is used in tests of significance and in determining confidence intervals. So, while the regression coefficient itself may be correctly estimated, an incorrect estimate of the standard error introduces inaccuracies in model testing and evaluation. Assumptions checking includes an evaluation of the reliability of independent variables, normality and homoscedasticity of residuals, and identification of multivariate outliers, which are discussed in more detail below. 


\section{Reliability of independent variables.}

Consistency of measurement for each predictor affects the strength of prediction of variance accounted for $\left(R^{2}\right)$. Internal reliability for each job characteristic dimension is calculated using Cronbach's $\alpha$ which measures how closely a set of items are related as a group. Consistent with measures of personality traits and attitudes, predictor reliabilities should fall within a 0.70 to 0.90 range.

\section{Normality and homoscedasticity of residuals.}

Residuals refer to the deviations of observed outcome values from their predicted values. As previously discussed, OLS estimates a line of best fit in which residuals are minimized - by estimating the line with residual distances equally located above and below. In data with no violations, these residual distances are equally dispersed across all levels of the predictors. This property of equality of variance in residuals is called homoscedasticity, whereas an unequal dispersion of residuals indicates an assumption violation called heteroscedasticity. Residual distances are also expected to have a normal distribution around the predicted regression line. While non-normally distributed or heteroscedastic residuals do not interfere with estimation of regression coefficients, they can lead to problems with calculation of standard error. Properties of residuals can be visually inspected by examining plots of residuals with their predicted values.

\section{Assumptions related to congruence research.}

As previously discussed in Chapter II, assumptions specific to polynomial regression analysis in congruence research include commensurate measures for predictors at interval or ratio level of measurement and scale-centered predictor terms (Caplan, 1987; Dawis \& Lofquist, 1984). 


\section{Commensurate measures.}

Commensurate dimensions ensure person and environment content is assessing the same construct to determine whether supplies exceed or fall short of needs in each content dimension. Specifically, congruence research involves commensurate person and environment dimensions through nominal and scale equivalence (Edwards \& Shipp, 2007). Nominal equivalence is achieved when person and environment are described in the same terms, such as for personally desired autonomy and the perception of autonomy being present in the job. Scale equivalence is obtained by assessing content dimensions on the same metric. For example, scale equivalence can be established by using the same response scale for person and environment, with different item stems to differentiate between person and environment.

\section{Scale-centered terms.}

Related to requirements for both polynomial regression and congruence research, predictors should to be centered prior to creating higher order terms for regression equations (Cohen et al., 2003). Depending on the research question, there are a variety of ways to center data, including mean-centered for regression interactions and group- or grand-mean centering for multilevel designs. Centering data at the midpoint of a scale, or scale-centered, is recommended for analysis of congruence (Edwards, 2002). Scale centering facilitates interpretation of coefficients on first-order terms when higher order terms are in the equation and may reduce multicollinearity between the component measures and their associated higher order terms (Cohen et al., 2003; Cronbach, 1987). Specifically, with scale centering, zero has been placed at the midpoint of $\mathrm{X}$ and $\mathrm{Y}$ scales allowing the model to estimate the slope of the surface at this meaningful point. Also, by 
centering predictors, multicollinearity that existed solely due to scaling is removed from the equation, allowing identification of multicollinearity associated with nonsymmetrical distribution of predictors.

\section{Multivariate outliers' detection.}

Outliers refer to a few extremely large or small scores relative to the full sample. Generally, outliers are problematic for estimation because of their effect on computations of descriptives and statistical tests (e.g., mean, standard deviation, regression coefficients). In multivariate models, there are three types of outliers, a) outliers related to independent variables (IV), b) outliers reflected by differences between predicted and observed outcomes, and c) outliers affecting the estimation of the model. However, while outliers can have a strong impact on estimated parameters and standard errors, they should not be simply discarded or removed from analyses.

Examination of multivariate outliers is accomplished by using indices of leverage, discrepancy, and influence to inform researchers about outliers that may impact model estimation. Visual inspection of index plots can also confirm which cases potentially change model estimation. In cases of influential outliers, estimation of the model with and without potential outlier cases should be conducted to make informed choices on how outliers should be handled.

\section{Leverage.}

Leverage refers to how unusual a case is in terms of its predictor values. Cases with greatest distance between their IV scores and the mean of IV scores have greater potential for influencing the estimation of a regression (Cohen et al., 2003). Centered leverage is a measure of distance for predictor variables that indicates cases furthest from 
the centroid, or point corresponding to the mean, of all predictors in the multivariate model (Cohen et al., 2003). Values greater than two times the mean identify the top 5\% of extreme values when all predictors are normally distributed (Belsley, Kuh, \& Welsch, 1980). While this cut-off value typically identifies too many cases, this method guides examination of index plots for cases with the highest leverage values (Cohen et al., 2003). Using an index plot of centered leverage by case number, those with relatively large gaps from the remaining cases are identified as outliers.

\section{Discrepancy.}

For the dissertation, externally studentized residual was used to identify extremity in distance between cases' observed values and their predicted values. Externally studentized residual value for each case is based on a regression not including that case. In other words, as an outlier can pull the regression line toward itself, externally studentized residuals consider the extremity of a predicted outcome value, without the influence of that case on the regression estimation. Externally studentized residuals follow a $t$ distribution with a cut-off of $|3.0|$ recommended for moderate-to-large sample sizes (Cohen et al., 2003). Cases identified as highly discrepant from their predicted values are then visually inspected using an index plot of externally studentized residuals by case number, with more attention given to cases with large gaps between themselves and the remaining cases.

\section{Influence.}

Influence reflects the amount that the regression coefficients would change if a case were removed (Cohen et al., 2003). Cases potentially having strong influence on regression were identified using standardized difference in fit (DFFITS) as a global 
influence indicator and standardized difference in beta coefficients estimation (DFBETAS) as an indicator of influence on each regression coefficient (Cohen et al., 2003). As a deletion technique, changes in the predicted model (DFFITS) or estimated regression coefficients (DFBETAS) are evaluated by running the regression with and without each case to determine how much influence each case contributes to the analysis. Typically, cases with DFFITS or DFBETAS magnitudes greater than $|1.0|$ were considered outliers. This determination was also visually confirmed by inspection of index plots of case number by DFFITS. For DFBETAS, index plots of case number by each DFBETAS value (i.e., for each regression coefficient) were examined. Cases with large DFFITS or DFBETAS values relative to other cases are more easily seen in these index plots as they are more distant from the majority.

Regression diagnostics are case statistics, meaning a value is generated for each case in the dataset. This diagnostic device was used to identify potential multivariate outliers in the dataset using SPSS to save values for centered leverage, externally studentized residuals, predicted outcome values, DFFITS and DFBETAS. Index plots between studentized residuals and standardized predicted values were also evaluated as they indicate potential violations of homoscedasticity.

\section{General model.}

To explore the dissertation's research questions, 20 models were individually analyzed using polynomial regression, with an evaluation of each model's resulting response surface. 
A general quadratic equation for these polynomial regression models is:

$$
Z=b_{0}+b_{1} X+b_{2} Y+b_{3} X^{2}+b_{4} X Y+b_{5} Y^{2}+e
$$

In Equation 12, $Y$ and $X$ represent needs and supplies, respectively, while $\mathrm{Z}$ represents the dependent variable of strain or well-being. Regression coefficients estimated by this model include the main effects of needs $\left(b_{2}\right)$ and supplies $\left(b_{1}\right)$, the interaction effect between needs and supplies $\left(b_{4}\right)$, and curvilinear effects of needs $\left(b_{5}\right)$ and supplies $\left(b_{3}\right)$. For the dissertation, which examines the relationship between preferred job characteristics $(J C P)$ and perceived present job characteristics $(J C E)$ with strain or well-being (Outcome), the same model would be:

$$
\text { Outcome }=b_{0}+b_{1} J C E+b_{2} J C P+b_{3} J C E^{2}+b_{4} J C E P+b_{5} J C P^{2}+e
$$

\section{Response surface methodology.}

Response surface methodology (Box \& Draper, 1987) provides a basis for estimating and interpreting three-dimensional surfaces corresponding to polynomial regression equations (Edwards, 2002; Edwards \& Parry, 1993) and was used to explore the dissertation's fit-outcome relationships (Shanock et al., 2010). Using polynomial regression and response surface analysis, several aspects of the fit-outcome relationship can be evaluated. First, person-job compatibility may have different relationships to an outcome depending on one's alignment of preference and presence. In other words, employees with a low preference for autonomy that is perfectly matched with their perception of low supply of autonomy in the job, may have a different outcome levels compared to employees with high needs in autonomy, even though these high needs were also matched by their perception of high supply of autonomy in the job. Recall that, fit 
refers to person-job compatibility represented by equal needs and supplies (e.g., low desire for autonomy coupled with low autonomy supplied in the job). As such, perfect fit can span the continuum from low preferred/present to high preferred/present for each job characteristics dimension. The examination of the nature of fit with an outcome focuses on this continuum along the line of fit.

Secondly, the nature of misfit (i.e., discrepancy, incompatibility, incongruence) between needs and supplies can also be examined in how misfit relates to an outcome (Shanock et al., 2010). As previously discussed, misfit represents the extent of disparity between needs and supplies. For example, a person might prefer to have decisionmaking latitude in work-related decisions but happens to be working in a structured job with step-by-step procedures allowing for little-to-no freedom in decision-making.

In instances of misfit, outcome levels may be different depending on which component (needs or supplies) is higher than the other. For example, when a misfit in decision latitude exists, decreases in satisfaction may be more pronounced when one prefers more decision latitude than perceived as available in the job (i.e., high preferred/low present), relatively compared to when one prefers less decision latitude but whose job requires more autonomous decision-making (i.e., low preferred/high present). In other words, the direction of discrepancy may have an effect on an outcome. Examination of the nature of misfit in relation to an outcome focuses on the line of misfit. Following is a brief description of the properties of the response surface, how linearity and curvilinearity of the lines of fit and misfit are tested, and how these attributes are used to interpret fit-outcome relationships. 


\section{Properties of the response surface.}

Estimated coefficients from the polynomial regression equation provide the information needed to plot its corresponding response surface. Response surfaces are comprised of the environment component of a job characteristic and the person component of the same job characteristic representing predictors on $\mathrm{X}$ and $\mathrm{Y}$ axes respectively, with the strain or well-being outcome as the dependent variable on the $Z$ axis (see Figure 11).

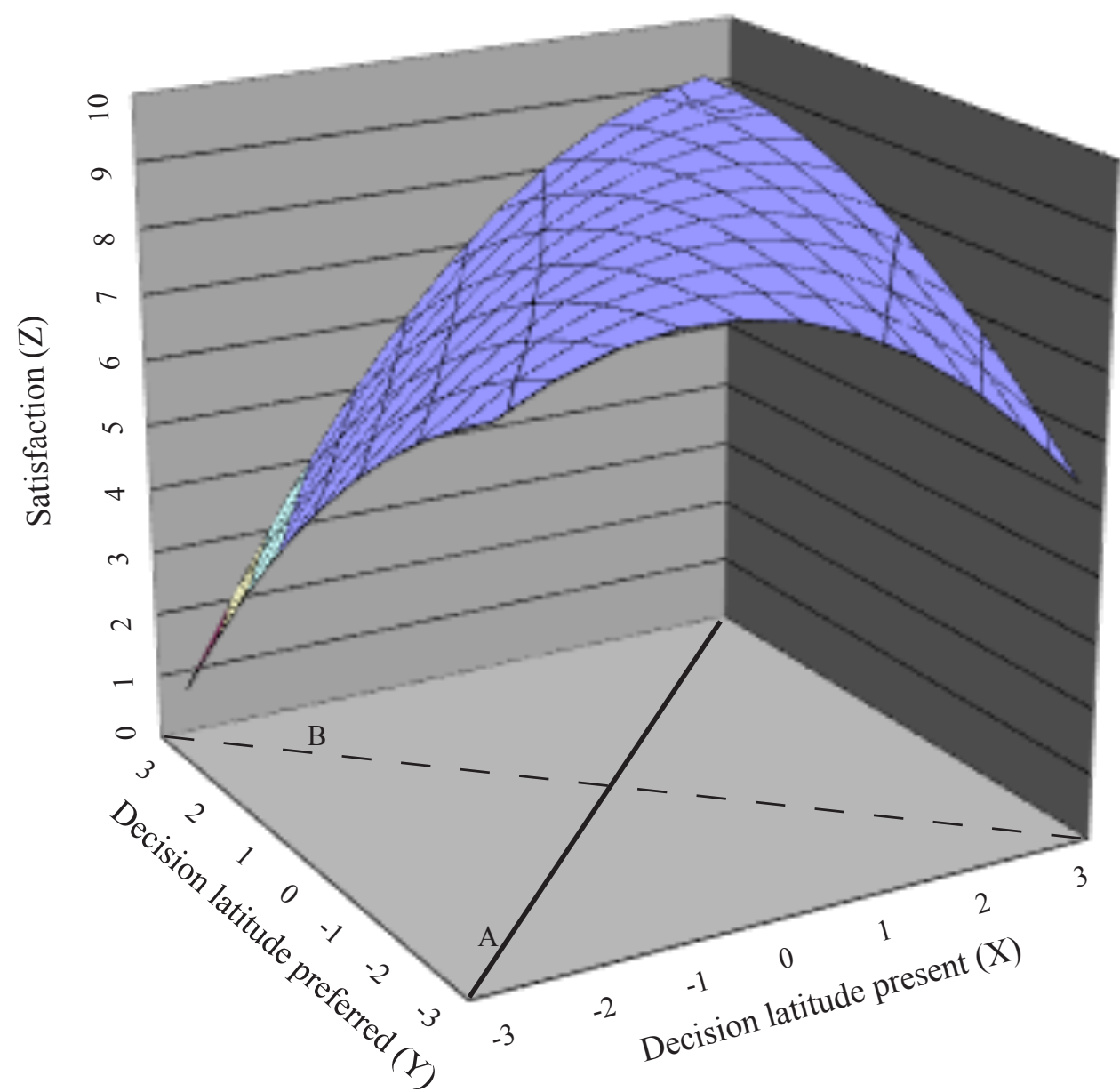

Figure 11. Response Surface. Illustration of hypothetical response surface showing relationship between decision latitude present $(\mathrm{X})$ and decision latitude preferred $(\mathrm{Y})$ with the outcome of satisfaction (Z). Base plot includes the solid line of fit (A) which runs from coordinates $(-3,-3)$ to $(3,3)$ and the dashed line of misfit (B) from coordinates $(3,-3)$ to $(-3,3)$. 
Lines of fit and misfit. Perfect congruence is represented by the line of fit along which $\mathrm{P}$ and $\mathrm{E}$ component measures are equal (e.g., preferred and perceived as present autonomy are equal; $\mathrm{Y}=\mathrm{X}$ ). Conversely, the line of misfit is perpendicular to perfect congruence spanning from opposite points of incongruence. Illustrated in Figure 11, the line of misfit has one end point where needs are high and supplies are low, with its other end point where needs are low and supplies are high (i.e., $Y=-\mathrm{X}$ ). Evaluation of the nature of fit and misfit is accomplished by describing the shape of the surface along the lines of fit and misfit, respectively.

Slope and curvature along line of fit. The slope of the line of fit $\left(a_{1}\right)$ indicates how compatibility between one's preferred and perceived present amount of a job characteristic is related to an outcome. Specifically, the slope along the line of fit indicates differences in outcome level while moving along the line of fit from low preferred/present to high preferred/present. Slope for the line of fit is determined by testing linear components of the regression equation $\left(a_{1}=b_{1}+b_{2}\right)$ where $b_{1}$ is the unstandardized beta coefficient for the environment component of a job characteristic dimension and $b_{2}$ is the unstandardized beta coefficient for the person component of that same job characteristic dimension. A significant positive slope indicates an increase in outcome levels as one moves along the line of fit. Conversely, a significant negative slope would indicate outcomes decrease as one moves from low preferred/present towards high preferred/present along the line of fit.

Curvature of the line of fit $\left(a_{2}\right)$ also provides information about the fit-outcome relationship surface and is calculated by sum of regression coefficients for non-linear terms $\left(a_{2}=b_{3}+b_{4}+b_{5}\right)$ where $b_{3}$ is the unstandardized beta coefficient for the squared 
term of the environment component of a specific job characteristic, $b_{4}$ is the unstandardized beta coefficient for the cross-product of person and environment components, and $b_{5}$ is the unstandardized beta coefficient for the squared term of the person component of the job characteristic. Curvature along the line of fit would indicate a sharp, non-linear increase or decrease in the outcome for low preferred/present fit that is not consistent for high preferred/present fit. For example, a positive curvilinear effect (i.e., U-shaped, convex) may indicate negligible increases in outcome levels as along the line of fit for employees with low-preferred/present fit to the midpoint, followed by a sharp increase from midpoint to high-preferred/present. A negative curvature would have an upside-down $\cap$-shape (i.e., concave) along the line of fit, possibly indicating a sharp increase in outcome levels at low-preferred/present, which then taper off for employees with high-preferred/present fit. While curvature of the line of fit is not typically seen (Shanock et al., 2010), the occurrence of significant curvature of the line of fit would affect the interpretation of the slope of the fit line. Specifically, slope of the fit line is estimated at the line's midpoint. If this line is also significantly curved, the slope at endpoints of the line of fit would not be consistent with the slope at the midpoint.

Slope and curvature along line of misfit. The relationship between degree of discrepancy to an outcome is described by the slope and curvature of the surface along the line of misfit. Perpendicular to the line of fit, the shape of the surface along the line of misfit indicates how discrepancy between preferred and perceived present job characteristics are related to the outcome. The slope along the line of misfit $\left(a_{3}\right)$ describes the relationship between direction of discrepancy with an outcome. For example, from left to right in Figure 11, the line of misfit describes a positive slope. A 
significant positive slope indicates that satisfaction is higher when misfit in decision latitude is such that perceived decision latitude in the job is higher than decision latitude preferred. This is in comparison to lower satisfaction for misfit indicated by decision latitude as preferred by employees but not perceived as present in the job. Slope for the line of misfit is calculated by testing the joint combination of regression coefficients of the linear terms along the line of misfit $\left(a_{3}=b_{1}-b_{2}\right)$.

A significant positive curvature of the misfit line $\left(a_{4}\right)$ describes a convex surface in which outcomes increase more sharply as the degree of discrepancy increased. The convex shape indicates minimized outcome levels closer to fit (e.g., minimized levels of burnout as discrepancy is reduced). Conversely, a significant negative curvature describes a concave surface in which outcomes sharply decrease as the degree of discrepancy increased. Therefore, a concave surface indicates maximized outcomes closer to fit (e.g., maximized satisfaction levels as discrepancy is reduced). The curvature of the misfit line is calculated by testing the joint combination of regression coefficients of the higher order terms along the line of misfit $\left(a_{4}=b_{3}-b_{4}+b_{5}\right)$.

Estimating properties of the surface. Significance testing and the construction of confidence intervals for estimates of slope and curvature of the fit and misfit lines (i.e., $a_{1}, a_{2}, a_{3}$, and $a_{4}$ ) require special attention. In OLS, regression coefficients have a sampling distribution that follows a $z$ or $t$ distribution. However, this is not true for expressions that are combinations of coefficients. These require sampling distributions derived by bootstrapping or jackknife procedures to accurately estimate standard errors (Edwards, 2002; Efron \& Tibshirani, 1994). 
Bootstrapping also serves to improve accuracy of ML estimation of the model parameters' standard errors (Enders, 2010). As previously discussed, multivariate normality is an important assumption for regression and estimation using ML. While non-normal data may have minimal impact on parameter estimation, they can bias standard errors and thereby affect accuracy of significance testing and confidence intervals estimation (Cohen et al., 2003; Enders, 2010). However, use of bootstrapping resampling reduces problematic normality violations in ML analyses by generating its own empirical sampling distribution for each parameter. In other words, bootstrap resampling technique makes no distributional assumptions; resulting in a procedure that is more robust to normality violations (Enders, 2010). A large number of samples (i.e., more than 2000) is suggested for potentially non-normal distributions (Enders, 2010). However, as 10,000 samples are recommended for estimation of slope and curvature of fit and misfit lines (Edwards, 2002), this accommodates the sample repetitions suggestion for normality violations.

\section{Power requirements.}

Statistical power refers to the probability of detecting an effect in a sample given that the effect does in fact exist in the population (Cohen, 1988) and is determined by sample size, effect size, measurement error, and significance level (Cohen et al., 2003). To determine sample size needed to detect fit-outcome relationships represented in the population, an alpha level of .05 was chosen as the cut-off value for significance, which is consistent with conventional research (Cohen et al., 2003). This represents the probability of finding a significant relationship in the sample that is truly not present in the population (i.e., Type I error). Determination of effect size was based on previous 
research examining the relationship between PE fit and strain outcomes. As previously stated, meta-analysis conducted by Kristof-Brown and colleagues (2005) found a moderate relationship between person-job fit and strain $(\hat{\rho}=-0.28)$, and more specifically between needs-supplies person-job fit and strain $(\hat{\rho}=-0.31)$. While studies included in their meta-analysis were predominantly based on difference scores or direct perception of fit, the values obtained for effect size were consistent with small-to-medium effects typically observed in social science (Cohen et al., 2003). G*Power3 (Faul, Erdfelder, Lang, \& Buchner, 2007) indicated a sample size of at least $N=136$ was required in order to have $80 \%$ power to detect a reasonable effect size $\left(r=-0.30 ; f^{2}=0.0989\right)$ for the five predictors including the interaction term. However, as individual predictors are less than perfectly reliable, the interaction term can be even more unreliable as the product of individual predictors. This may reduce the power to detect effects of the interaction term relative to the power to detect first order effects (Cohen et al., 2003). Therefore, a sample size of 200 was targeted to account for unreliability and interaction of predictors in the regression model.

\section{Summary of Data Analysis Plan}

In summary, the dissertation focused on strain and well-being outcomes for emergency responders across several branches. Emergency responders in the United States were recruited using snowball sampling for data collection via online survey. As detailed in the Data Analysis Plan, person mean imputation was used to handle missing data on outcome variables having at least $80 \%$ item-level data, resulting in 358 cases used in analyses. After examination of assumptions, ML and bootstrapping techniques were used to address remaining missing data issues at construct-level, estimate standard 
errors for linear combinations, and model relationships between job characteristics and strain or well-being outcomes. Results of these fit-outcome relationships are presented in Chapter IV.

While an exhaustive examination of the surface might result in conclusions too nuanced to be effective in organizational interventions, these explorations of the dataspace serve to forward our theoretical understanding of these phenomena. Properties of the response surface were evaluated to understand the relationship between variations in fit and misfit with strain or well-being outcomes. 


\section{CHAPTER IV: RESULTS}

\section{Treatment of Missing Data}

Upon survey closure, Qualtrics had logged 653 cases in which a participant had started the survey. However, 295 nonrespondents were removed, retaining 323 partial respondents and 35 full respondents, which resulted in construct-level missingness ranging from $25 \%$ to $57 \%$. Little's MCAR test was significant, indicating data were not $\operatorname{MCAR}[\chi 2(4791)=1363.37, p=1.0]$. It is likely that missing data are MAR, not due to specific variables in the study, but due to survey fatigue (Porter, Whitcomb, \& Weitzer, $2004)$ as the highest percentage of missing values (57\%) occurred towards the end of the survey. Item-level missingness for outcome scales was handled by computing scale scores by average for all cases with at least $80 \%$ completion, which resulted in $8.1 \%$ construct-level missingness for PSI and $0.3 \%$ construct-level missingness for burnout, secondary traumatic stress, and compassion satisfaction in ProQOL.

The chosen approach to missing data retained the most cases $(N=358)$, while allowing opportunities to use missing data techniques to account for missingness. Maximum likelihood was used to estimate model parameters such that even constructs with less than $80 \%$ completion could still contribute to model estimation with the partial information that was available (Newman, 2014).

\section{Descriptives}

Relationships among study variables including main predictors (i.e., person and environment components for each job characteristic), outcomes of interest, potential demographic covariates, and Cronbach's $\alpha$ are reported in Table A3, Appendix A. 
Scatterplots for predictor space (i.e., person and environment components for each job characteristic) are provided in Figure 12.
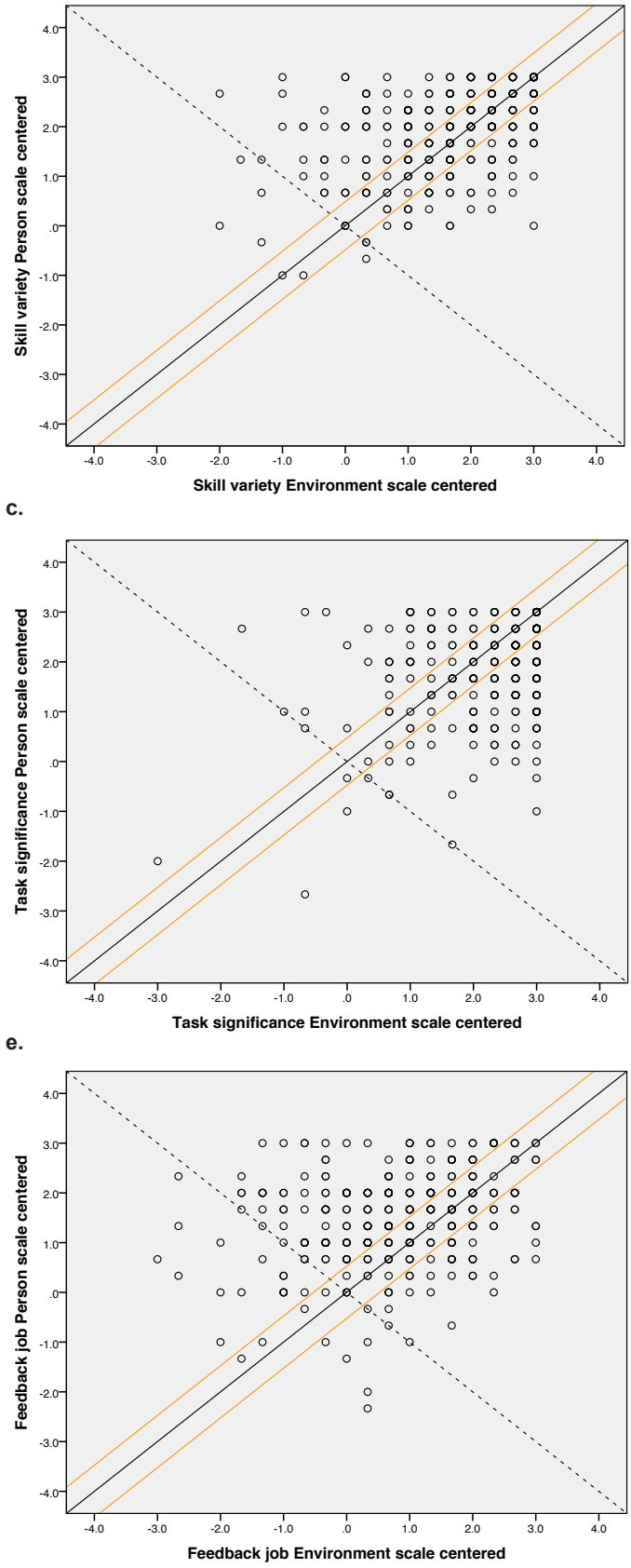

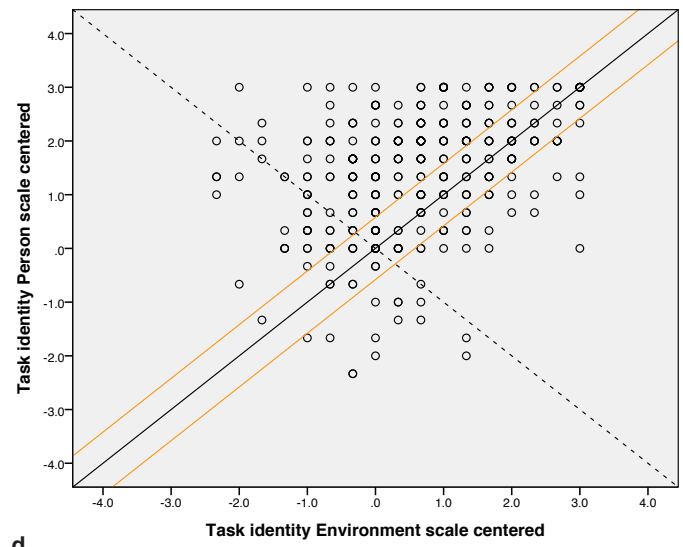

d.

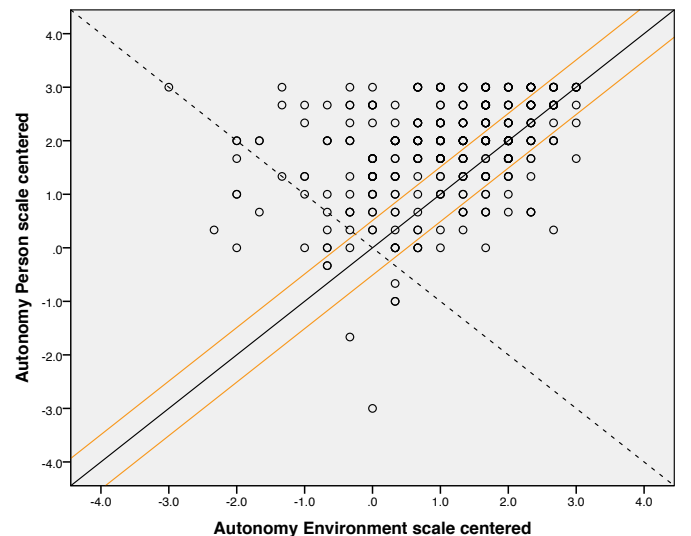

Solid line indicates fit line (i.e.,agreement) Dashed line indicates misfit line (i.e., discrepancy)

Orange lines indicate $+/-.5$ standard deviation

Figure 12. Scatter Plot of Predictor Space illustrates distribution for each job characteristics dimension with environment component on $\mathrm{x}$ axis and person component on y axis as follows: (a) skill variety, (b) task identity, (c) task significance, (d) autonomy, and (e) feedback from the job. 


\section{Model Testing}

\section{Scale centering and higher order terms.}

Main effects predictors were scale-centered by subtracting the scale midpoint (4) to produce values ranging from -3 to 3 (Edwards, 2002). Interaction and curvilinear terms were computed using these scale-centered values.

\section{Identification of multivariate outliers.}

As discussed in Data Analysis Plan, centered leverage, externally studentized residual, and DFFITS/DFBETAS diagnostics were used to identify outliers in predictor space, outcome discrepancy, and model estimation respectively. Four cases were identified as potential outliers in the dataset $(118,130,215$, and 291), which were confirmed by visual inspection of their respective index plots of case number by diagnostic. Cases were examined for contamination (e.g., errors in scale calculation, careless response) with no specific contamination issues identified. As previously discussed, analyses of fit-outcome model parameters and surface properties were tested using ML with bootstrapping (10,000 samples; Edwards, 2002) using Mplus V7.2 (Muthén \& Muthén, 2012). This approach to model testing provided similar results with or without outlier cases. Therefore, all cases were retained for analyses (Judd, McClelland, \& Culhane, 1995).

\section{General model.}

$$
\text { Outcome }=b_{0}+b_{1} J C E+b_{2} J C P+b_{3} J C E^{2}+b_{4} J C E P+b_{5} J C P^{2}+e
$$

As discussed in Chapters II and III, the polynomial regression model posits that the level of an outcome is explained by the joint effects of (a) linear main effects of a specific job characteristic in person and environment components, (b) interaction effects 
indicated by the product of person and environment terms, and (c) curvilinear effects indicated by squared person and squared environment terms, plus error. For each job characteristics dimension, regression results and corresponding response surface were examined for nature of fit and misfit with each outcome variable.

\section{Nature of fit.}

To examine the impact of agreement between one's preference for and perceived presence of a job characteristics dimension with an outcome, linearity and curvilinearity of the line of fit were tested. As discussed in the Data Analysis Plan section, the shape of the surface along the line of fit can be found by substituting within the regression equation the condition of $\mathrm{Y}=\mathrm{X}$. This is accomplished by using the MODEL CONSTRAINT command in MPlus (Muthén \& Muthén, 2012), such that combinations of coefficients for linear and curvilinear terms are jointly tested (Edwards, 2002; Edwards \& Parry, 1993). Specifically, a combination of linear regression coefficients ( $b_{1}$ and $b_{2}$ in the General Model $)$ was tested for significant slope $\left(a_{1}=b_{1}+b_{2}\right)$ and curvilinear regression coefficients $\left(b_{3}, b_{4}\right.$, and $\left.b_{5}\right)$ were combined and tested for significant curvature $\left(a_{2}=b_{3}+b_{4}+b_{5}\right)$.

\section{Nature of misfit.}

To examine the impact of discrepancy between one's preferred and perceived present for each job characteristics dimension, tests of linearity and curvilinearity were conducted for the line of misfit (i.e., the line perpendicular to the line of fit; $Y=-X$ ). Similar to prior testing of the nature of fit, MODEL CONSTRAINT command was used to assign names to combinations of regression coefficients and test these joint combinations for significant linearity and curvilinearity along the line of misfit. 
Estimated regression coefficients were combined such that linear regression coefficients were subtracted $\left(a_{3}=b_{1}-b_{2}\right)$ to test for significant slope of the misfit line; and curvilinear regression coefficients were combined with the interaction regression coefficient subtracted $\left(a_{4}=b_{3}-b_{4}+b_{5}\right)$ to test for significant curvature of the misfit line. Syntax for MODEL CONSTRAINT commands provided in Appendix C.

Results are organized by job characteristics dimensions (i.e., skill variety, task identity, task significance, autonomy, and job-based feedback) to report model testing for each fit-outcome relationship. For each job characteristic, the following sequence is used according to the four outcomes of interest: (1) physical symptoms, (2) burnout, (3) secondary traumatic stress, and (4) compassion satisfaction.

\section{Skill Variety}

Two constructs assessed participants' experience of skill variety. Skill variety environment ( $\mathrm{SvE}$ ) assessed level of skill variety one perceived as present or supplied by their job, whereas skill variety person (SvP) indicated how much variety one preferred in their job. On a 7-point scale with higher values indicating more skill variety present, SvE $\left(N=358, M=5.66, M_{s c}=1.66, S D=1.06, s^{2}=1.12\right)$ had a low reliability of .592 , with negative skew (-0.856) and positive kurtosis (0.484). Likewise, reliability for $\mathrm{SvP}(N=$ $\left.358, M=5.91, M_{s c}=1.91, S D=0.86, s^{2}=0.75\right)$ was .608 with a slight negative skew ($0.768)$ and positive kurtosis (0.117). Of note, SvP and SvE were both significantly related to outcomes of burnout and compassion satisfaction. However, SvP was the only predictor related to secondary traumatic stress, and SvE was the only predictor related to physical symptoms (see Table A3). 


\section{Skill variety fit to physical symptoms relationship.}

Overall, the regression analysis of skill variety fit to physical symptoms resulted in small effect $\left(R^{2}=.020\right)$ with non-significant linear and curvilinear regression coefficients (Figure 13; Table A4).

\section{Nature of fit.}

Response surface analysis resulted in a non-significant linear slope along the line of fit $\left(a_{1}=-0.123, n s\right)$ and a non-significant curvilinear effect $\left(a_{2}=-0.350, n s\right)$. In respect to the outcome, non-significant slope and curvature indicates no differential relationship existing between physical symptoms along the skill variety fit line. In other words, as one moves along the line of fit from low preferred/present skill variety to high preferred/present skill variety, no significant difference in frequency for physical symptoms was identified (Edwards, 2002; Shanock et al., 2010).

\section{Nature of misfit.}

Results indicated non-significant slope and curvature of the surface along the line of misfit $\left(a_{3}=-0.003, n s ; a_{4}=1.034, n s\right)$. Skill variety misfit between preferred and present skill variety was not significantly related to frequency of physical symptoms. Overall, results indicate skill variety fit does not have a significant effect on physical symptoms. 


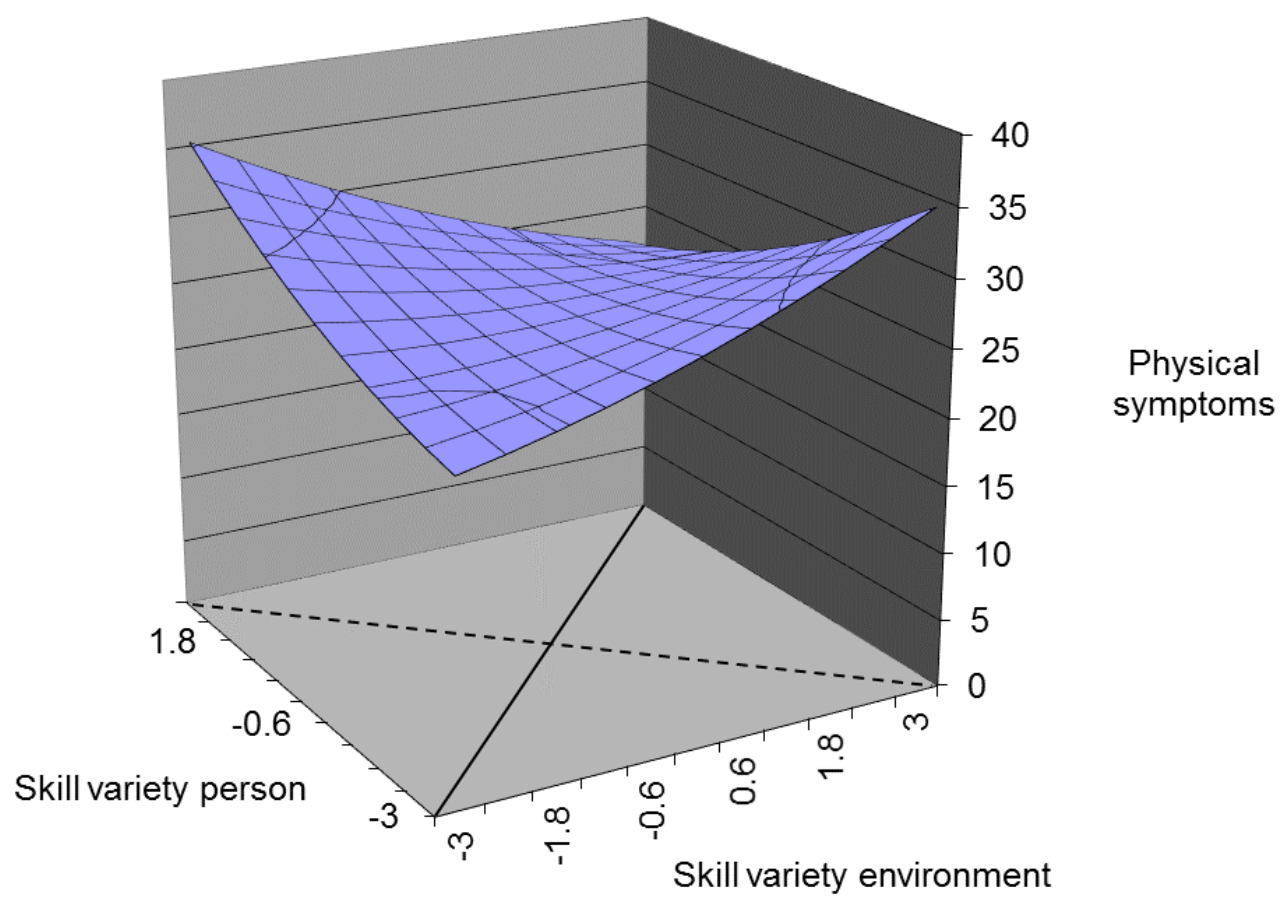

Figure 13. Skill Variety Fit with Physical Symptoms. Response surface for skill variety fit and outcome of physical symptoms. Solid line indicates line of fit; dashed line indicates line of misfit.

\section{Skill variety fit to burnout relationship.}

Overall, regression analysis for skill variety fit on burnout resulted in a moderate effect $\left(R^{2}=.102\right)$. Estimated regression coefficients for linear, interaction, and curvilinear terms were non-significant (Figure 14; Table A5).

\section{Nature of fit.}

Response surface analysis resulted in a non-significant linear slope along the line of congruence $\left(a_{1}=-0.166, n s\right)$ and a non-significant curvilinear effect $\left(a_{2}=0.032, n s\right)$. In respect to the outcome, a non-significant slope indicates no significant relationship exists between levels of burnout experienced and skill variety along the line of fit. In other words, as one moves along the line of fit from low preferred/present skill variety to 
high preferred/present skill variety, there were no significant differences in burnout levels (Edwards, 2002; Shanock et al., 2010).

\section{Nature of misfit.}

Similarly, results indicated non-significant slope and curvature of the surface along the line of misfit $\left(a_{3}=-0.060, n s ; a_{4}=-0.084, n s\right)$. In other words, discrepancy between preferred and present skill variety was not significantly related to burnout. Overall, results indicated skill variety fit does not have a significant effect on burnout.

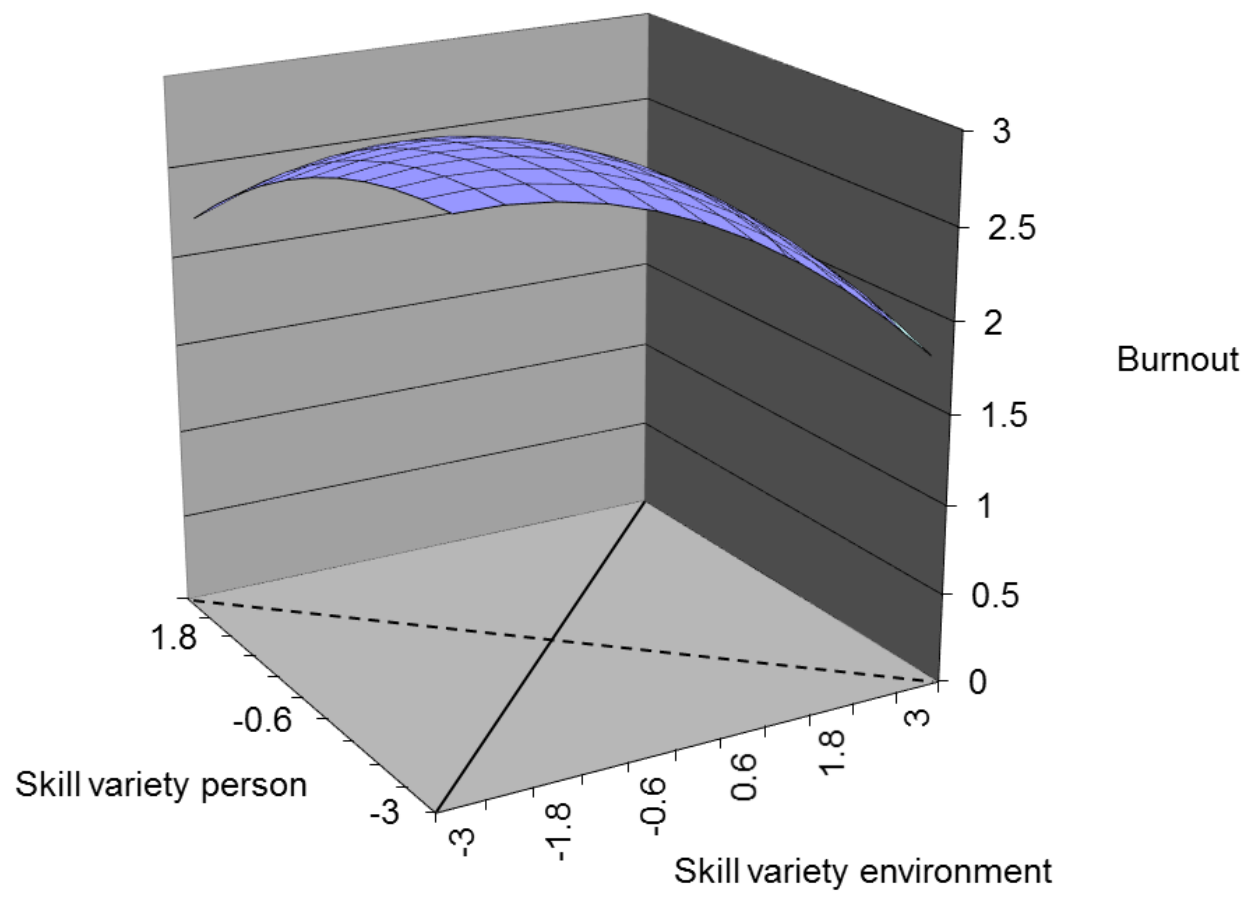

Figure 14. Skill Variety Fit with Burnout. Response surface for skill variety fit and outcome of burnout. Solid line indicates line of fit; dashed line indicates line of misfit.

\section{Skill variety fit to secondary traumatic stress relationship.}

Polynomial regression results evaluating skill variety fit with secondary traumatic stress found a small effect $\left(R^{2}=.020\right)$ with no significance found for linear, interaction, or curvilinear regression coefficients (Figure 15; Table A6). 


\section{Nature of fit.}

To examine the nature of skill variety fit related to levels of secondary traumatic stress, linearity and curvilinearity of the line of fit were tested. This analysis resulted in a non-significant linear slope along the line of congruence $\left(a_{1}=-0.198, n s\right)$ and a nonsignificant curvilinear effect $\left(a_{2}=0.025, n s\right)$. In respect to the outcome, a non-significant slope indicates no significant changes in secondary traumatic stress were found along the line of skill variety fit. In other words, from low preferred/present skill variety to high preferred/present skill variety, there were no changes in experienced secondary traumatic stress levels reported (Edwards, 2002; Shanock et al., 2010).

\section{Nature of misfit.}

To examine the nature of misfit in skill variety (as related to levels of secondary traumatic stress), similar tests of linearity and curvilinearity of the line of misfit were performed. Results indicated non-significant slope and curvature of the surface along the line of misfit $\left(a_{3}=0.142, n s ; a_{4}=-0.081, n s\right)$. In other words, discrepancy between preferred and present skill variety was not significantly related to secondary traumatic stress. Overall, results indicated skill variety fit does not have a significant effect on secondary traumatic stress. 


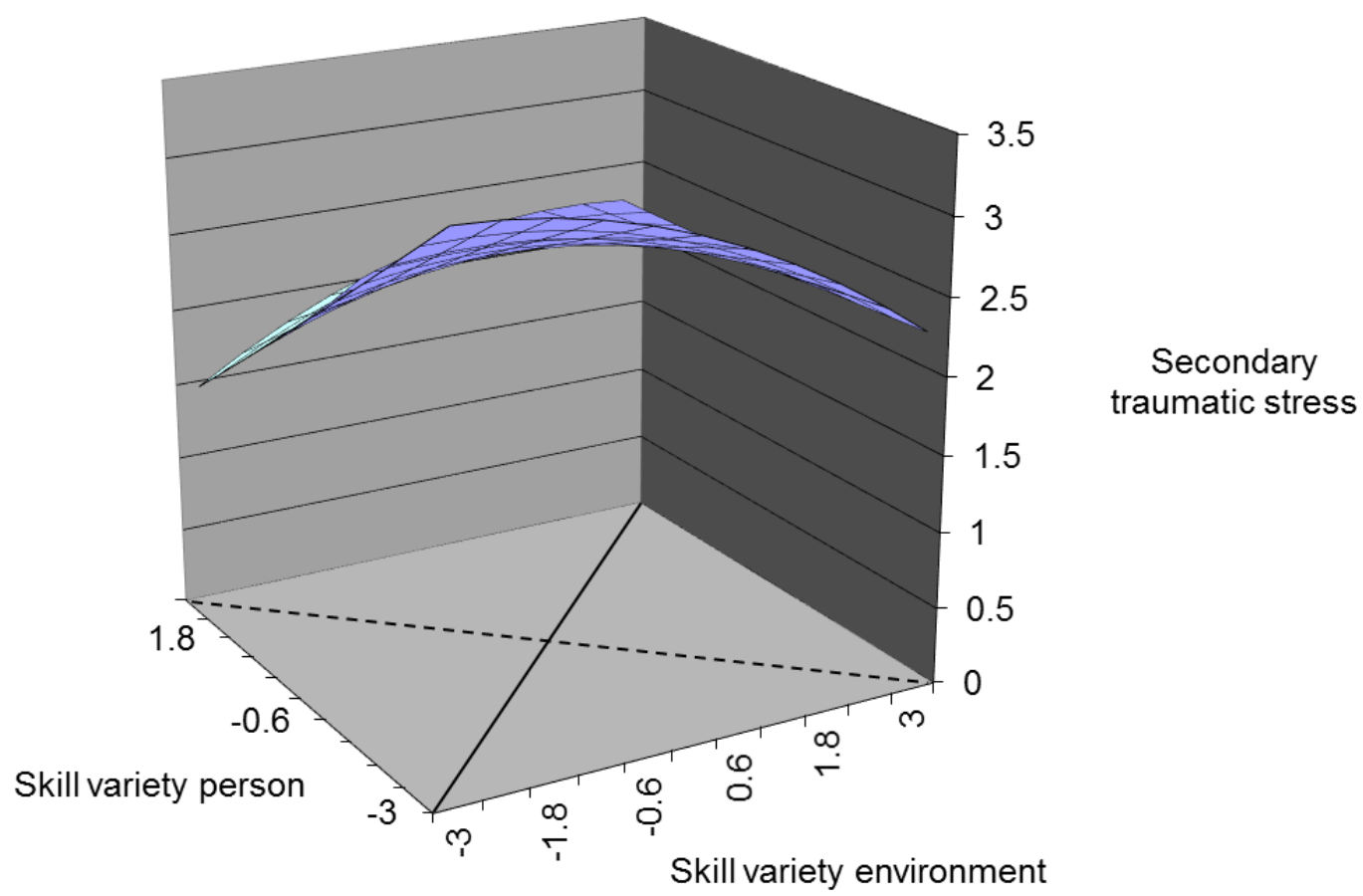

Figure 15. Skill Variety Fit with Secondary Traumatic Stress. Response surface for skill variety fit and outcome of secondary traumatic stress. Solid line indicates line of fit; dashed line indicates line of misfit.

\section{Skill variety fit to compassion satisfaction relationship.}

Regression analysis of skill variety fit with compassion satisfaction found a strong effect $\left(R^{2}=.237\right)$ with a significant regression coefficient for SvE (Figure 16; Table A7).

\section{Nature of fit.}

Response surface analysis resulted in a significant positive linear slope along the line of fit $\left(a_{1}=0.366, p<.05\right)$, but a non-significant curvilinear effect $\left(a_{2}=0.012, n s\right)$. In fact, compassion satisfaction was the only outcome with significant response surface properties associated with skill variety fit. Specifically, compassion satisfaction levels significantly increased along the skill variety line of fit from low preferred/present to high preferred/present. 


\section{Nature of misfit.}

To examine the nature of misfit in skill variety (as related to levels of compassion satisfaction), similar tests of linearity and curvilinearity of the line of misfit were conducted. Results indicated non-significant slope and curvature of the surface along the line of misfit ( $\left.a_{3}=0.203, n s ; a_{4}=0.120, n s\right)$. In other words, discrepancy between preferred and present skill variety was not significantly related to levels of compassion satisfaction.

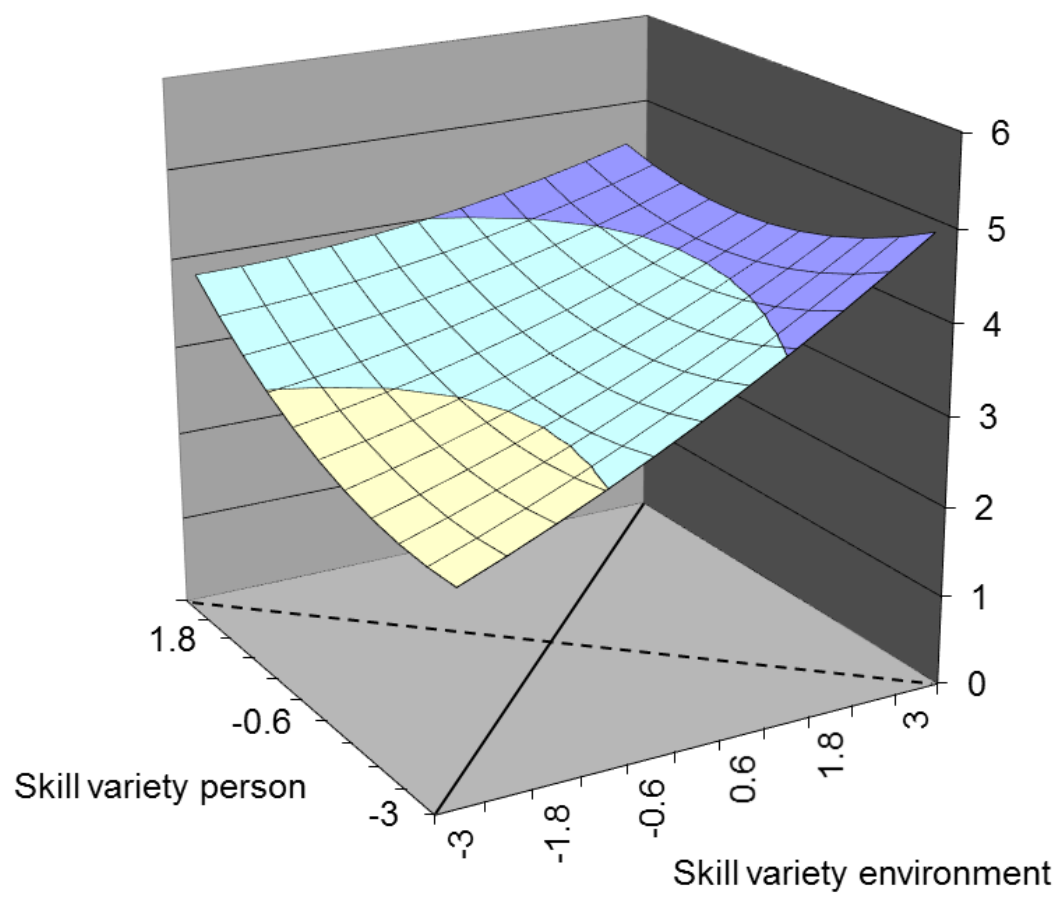

Figure 16. Skill Variety Fit with Compassion Satisfaction. Response surface for skill variety fit and outcome of compassion satisfaction. Solid line indicates line of fit; dashed line indicates line of misfit. 


\section{Task Identity}

As previously discussed, task identity refers to the extent one identifies with the result of their work efforts. This was assessed in how much task identity employees prefer (TiP) and how much task identity employees perceive in their job (TiE). Using a 7point scale in which higher values indicated more task identity preferred or present in the job, $\operatorname{TiP}\left(N=358, M=5.40, M_{s c}=1.40, S D=1.13, s^{2}=1.29\right)$ had a reliability of .825, with a negative skew (-0.73) and positive kurtosis $(0.33)$. TiE $\left(N=358, M=4.62, M_{s c}=\right.$ $\left.0.62, S D=1.18, s^{2}=1.38\right)$ had a low reliability of .533 , with positive skew $(0.05)$ and negative kurtosis (-0.32). Of note, TiE was significantly related to all outcomes, while the TiP was not related to any outcomes (see Table A3).

\section{Task identity fit to physical symptoms relationship.}

Regression analysis to evaluate take identity fit with physical symptoms found a moderate effect $\left(R^{2}=.071\right)$. However, individual regression coefficients were not significantly predictive of physical symptoms (Figure 17; Table A8).

\section{Nature of fit.}

Results of the polynomial analysis found a non-significant linear slope effect $\left(a_{l}\right.$ $=-0.673, n s)$ and a non-significant curvilinear effect $\left(a_{2}=-0.463, n s\right)$ along the line of fit. In respect to the outcome, a non-significant slope indicates no significant relationship exists between physical symptoms and task identity along the fit line. In other words, from low preferred/present task identity fit to high preferred/present task identity fit, there were no changes in frequency of physical symptoms (Edwards, 2002; Shanock et al., 2010). 


\section{Nature of misfit.}

Similarly, results to examine misfit in task identity indicated non-significant slope and curvature of the surface along the line of misfit $\left(a_{3}=-0.775, n s ; a_{4}=0.675, n s\right)$. In other words, degree of misfit between preferred and present task identity was not significantly related to frequency of physical symptoms. Overall, results indicated task identity fit does not have a significant effect on physical symptoms.

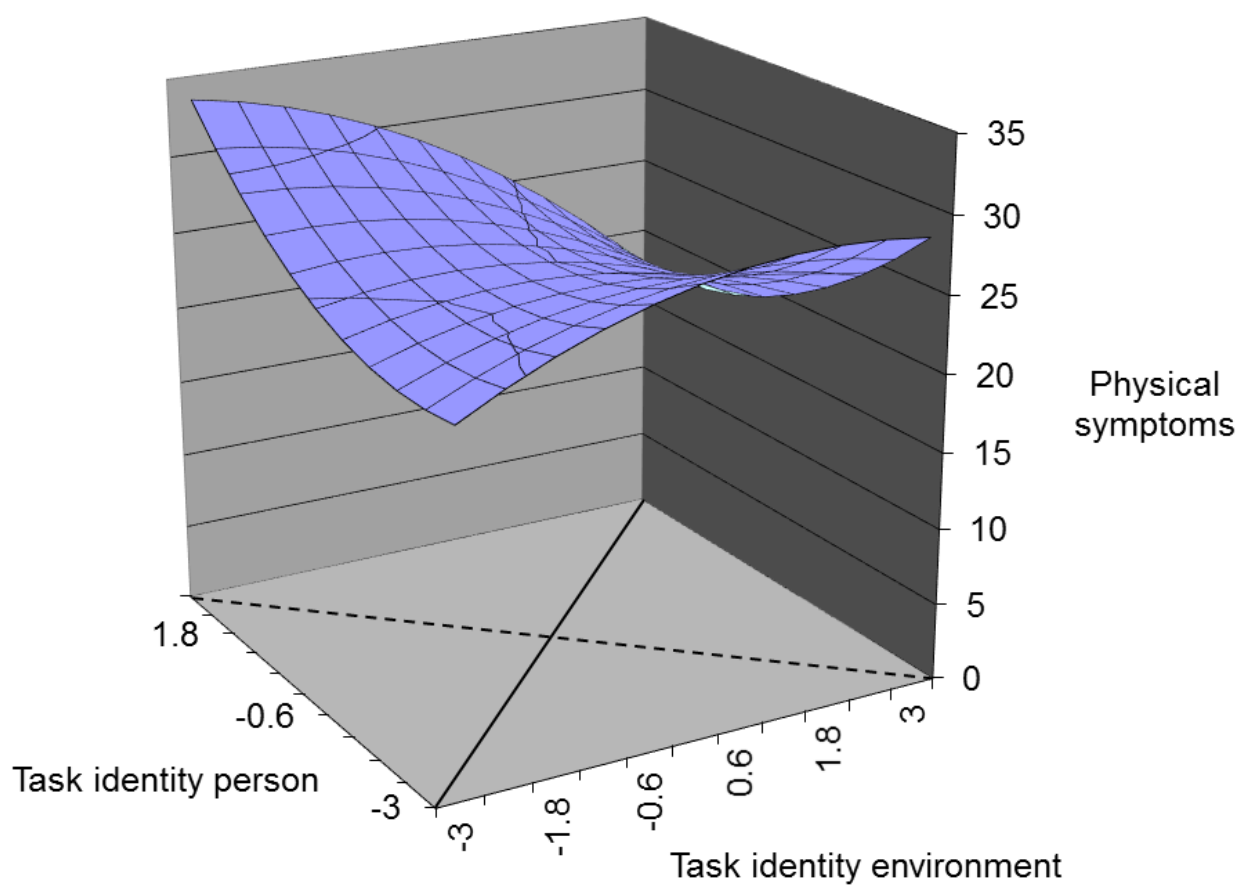

Figure 17. Task Identity Fit with Physical Symptoms. Response surface for task identity fit and outcome of physical symptoms. Solid line indicates line of fit; dashed line indicates line of misfit. 


\section{Task identity fit to burnout relationship.}

Polynomial regression of task identity fit with burnout found a moderate effect $\left(R^{2}=.085\right)$ with the person component of task identity as a significant predictor of burnout (Figure 18; Table A9).

\section{Nature of fit.}

This analysis resulted in a non-significant linear slope along the line of fit $\left(a_{1}=\right.$ $0.096, n s)$, but a significant negative curvilinear effect $\left(a_{2}=-0.083, p<.05\right)$. In respect to the outcome, a negative curvilinear effect along the line of fit indicates lowest levels of burnout for fit at the two extremes of the fit line continuum. In other words, burnout was lowest when task identity fit was at low preferred/present and high preferred/present positions of agreement along the line of fit. Emergency responders reported higher levels of burnout at the mid-point of fit (i.e., moderate preferred/present).

\section{Nature of misfit.}

Results to examine the nature of misfit in task identity indicated non-significant slope and curvature of the surface along the line of misfit $\left(a_{3}=-0.137, n s ; a_{4}=0.087, n s\right)$. In other words, discrepancy between preferred and present task identity was not significantly related to levels of burnout. 


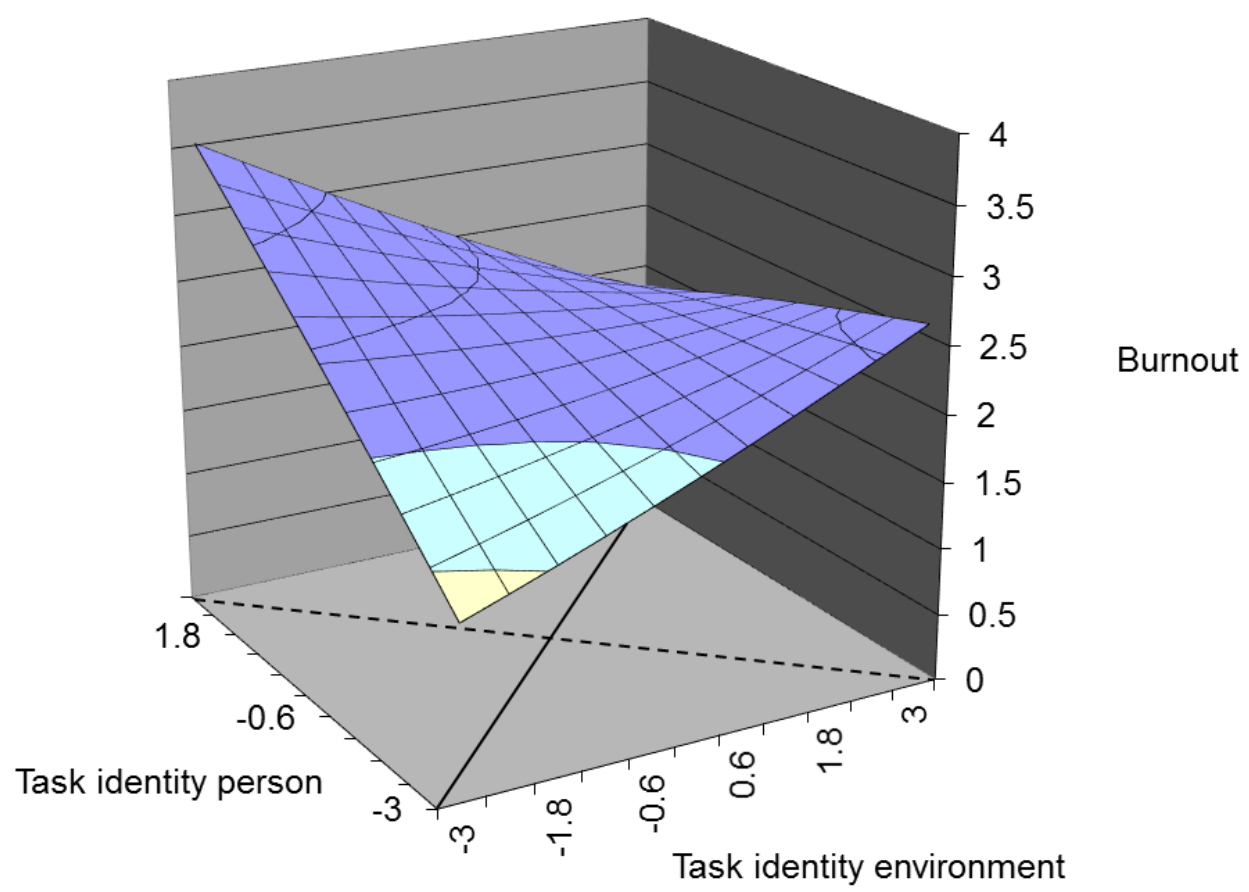

Figure 18. Task Identity Fit with Burnout. Response surface for task identity fit and outcome burnout. Solid line indicates line of fit; dashed line indicates line of misfit.

\section{Task identity fit to secondary traumatic stress relationship.}

Regression analysis to examine task identity fit with secondary traumatic stress found a strong effect for the model $\left(R^{2}=.102\right)$. Estimated regression coefficient for TiP was found significantly predictive of secondary traumatic stress (Figure 19; Table A10).

\section{Nature of fit.}

Both slope and curvature along the line of fit were non-significant $\left(a_{1}=0.074, n s\right.$; $\left.a_{2}=-0.056, n s\right)$. In respect to the outcome, this indicated no differences in one's experience of secondary traumatic stress along the line of fit for task identity. In other words, one's experience of secondary traumatic stress was not related to whether task identity fit was at low preferred/present, moderate preferred/present, or high preferred/present positions of agreement. 


\section{Nature of misfit.}

Tests of linearity and curvilinearity of the line of misfit indicated a significant linear effect $\left(a_{3}=-0.209, p<.05\right)$ and a non-significant curvilinear effect $(a 4=0.065$, $n s)$. A significant linear slope on the line of misfit specifies how the direction of discrepancy between preferred and present task identity is related to the outcome (Shanock et al, 2010). Specifically, the negative slope indicated that higher levels of secondary traumatic stress were experienced when preference for task identity was greater than task identity perceived as present in the job.

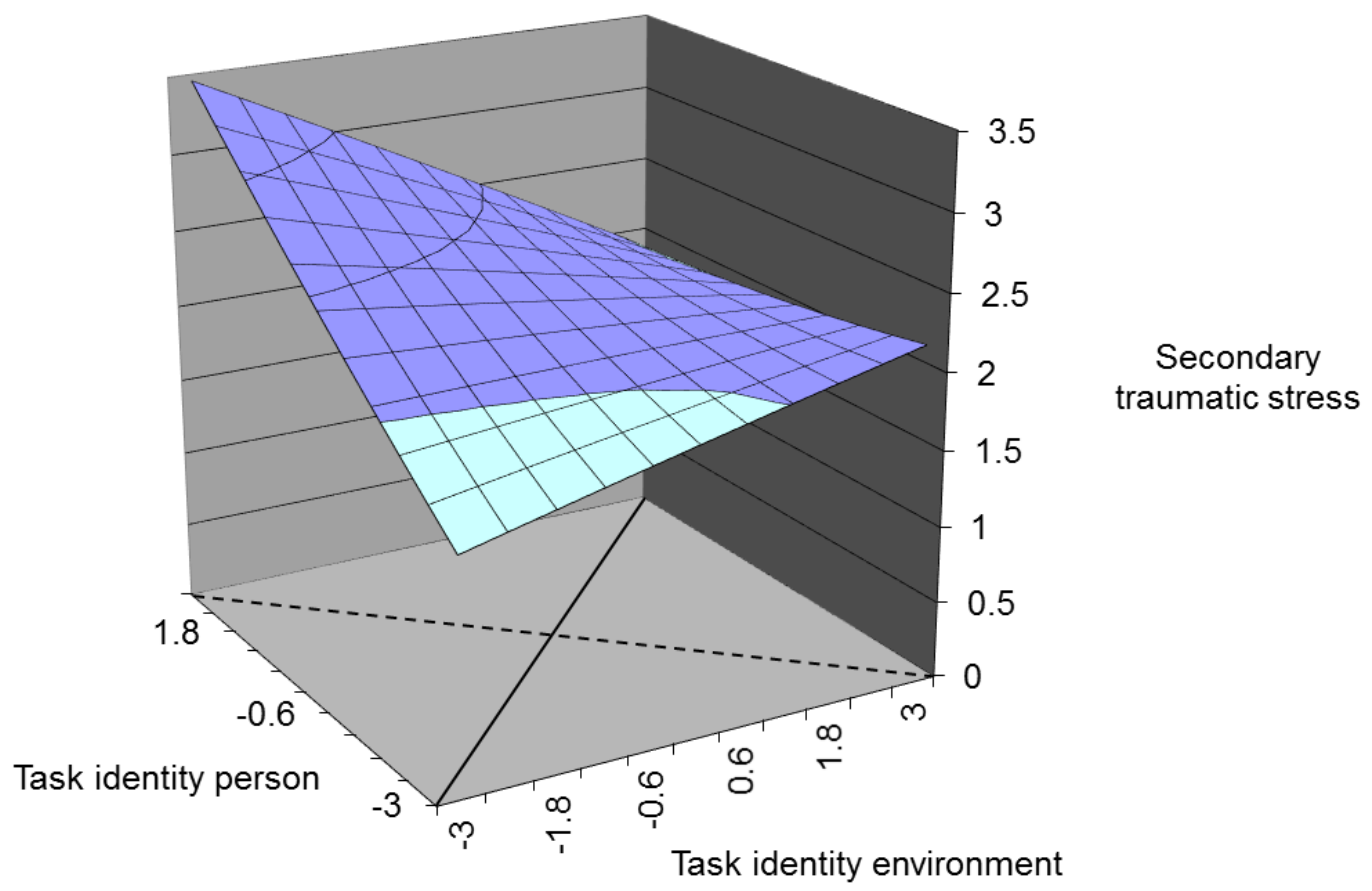

Figure 19. Task Identity Fit with Secondary Traumatic Stress. Response surface for task identity fit and outcome secondary traumatic stress. Solid line indicates line of fit; dashed line indicates line of misfit. 


\section{Task identity fit to compassion satisfaction relationship.}

Polynomial regression of task identity fit with compassion satisfaction found a small-to-moderate effect $\left(R^{2}=.041\right)$ with no significance for any estimated regression coefficients (Figure 20; Table A11).

\section{Nature of fit.}

Analysis of the response surface resulted in a non-significant linear slope $\left(a_{l}=-\right.$ $0.123, n s)$ and a non-significant curvilinear effect $\left(a_{2}=0.058, n s\right)$ along the line of fit. In respect to the outcome, a non-significant slope indicates no significant relationship exists between compassion satisfaction and task identity agreement. In other words, along the line of fit from low preferred/present task identity to high preferred/present task identity, there were no differences in compassion satisfaction reported (Edwards, 2002; Shanock et al., 2010).

\section{Nature of misfit.}

Examination of the nature of misfit in task identity (as related to levels of compassion satisfaction) found non-significant slope and curvature of the surface along the line of misfit $\left(a_{3}=0.097, n s ; a_{4}=-0.037, n s\right)$. In other words, discrepancy between preferred and present task identity was not significantly related to compassion satisfaction. Overall, results indicated task identity fit does not have a significant effect on compassion satisfaction. 


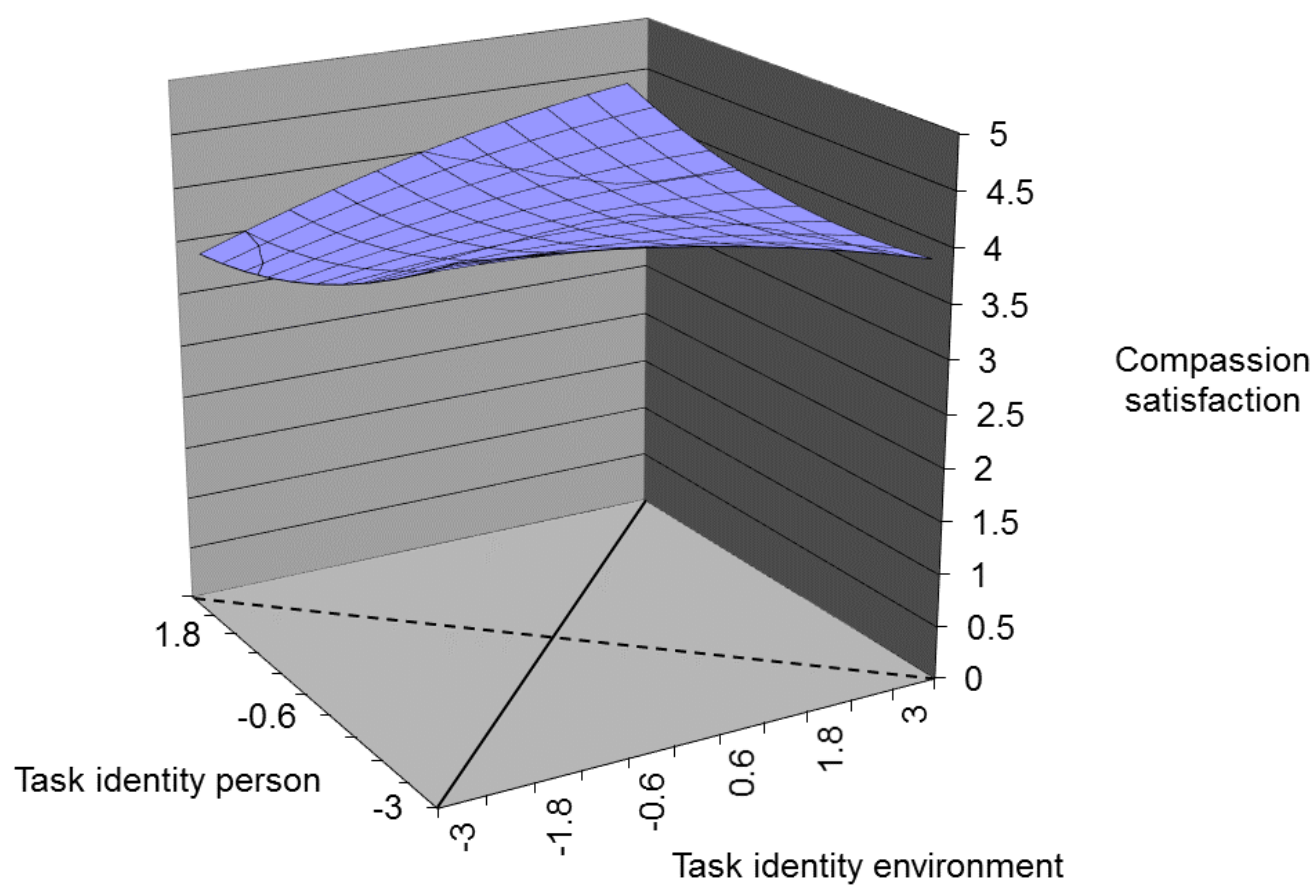

Figure 20. Task Identity Fit with Compassion Satisfaction. Response surface for task identity fit and outcome compassion satisfaction. Solid line indicates line of fit; dashed line indicates line of misfit.

\section{Task Significance}

Task significance, or one's perception of their job having an impact on the lives of others, was assessed in the two person-environment components: how much task significance employees perceive as present in their job (TsE) and how much task significance is preferred (TsP). On a 7-point scale in which higher values indicated more task significance present or preferred, $\operatorname{TsE}\left(N=358, M=6.31, M_{s c}=2.31, S D=0.920, s^{2}\right.$ $=0.847)$ had significant negative skewness (-1.889) and kurtotic (4.555) properties. Internal reliability for TsE was calculated at .615. Likewise, $\mathrm{TsP}\left(N=358, M=5.96, M_{s c}\right.$ $\left.=1.96, S D=0.976, s^{2}=0.953\right)$ was negatively skewed (-1.257) and kurtotic (2.082), with reliability at .643 . TsE was significantly related to all outcomes except for secondary 
traumatic stress. However, TsP was only significantly related to secondary traumatic stress and burnout (see Table A3).

\section{Task significance fit to physical symptoms relationship.}

Polynomial regression indicated a small effect $\left(R^{2}=.034\right)$ for task significance fit with physical symptoms. None of the estimated regression coefficients for task significance were significantly predictive of physical symptoms (Figure 21; Table A12).

\section{Nature of fit.}

Response surface analysis resulted in a non-significant linear slope $\left(a_{1}=-3.414\right.$, $n s)$ and a non-significant curvilinear effect $\left(a_{2}=0.581, n s\right)$ along the line of fit. In respect to the outcome, a non-significant slope indicates no significant relationship exists between physical symptoms and task significance fit along the line of fit. In other words, as one follows the task significance line of fit from low preferred/present to high preferred/present, there were no changes in frequency of physical symptoms reported (Edwards, 2002; Shanock et al., 2010).

\section{Nature of misfit.}

Results for examination of task significance misfit with physical symptoms also indicated non-significant slope and curvature of the surface along the line of misfit $\left(a_{3}=-\right.$ $\left.3.041, n s ; a_{4}=0.088, n s\right)$. In other words, discrepancy between preferred and present task significance was not significantly related to frequency of physical symptoms. Overall, results indicated task significance fit does not have a significant effect on physical symptoms. 


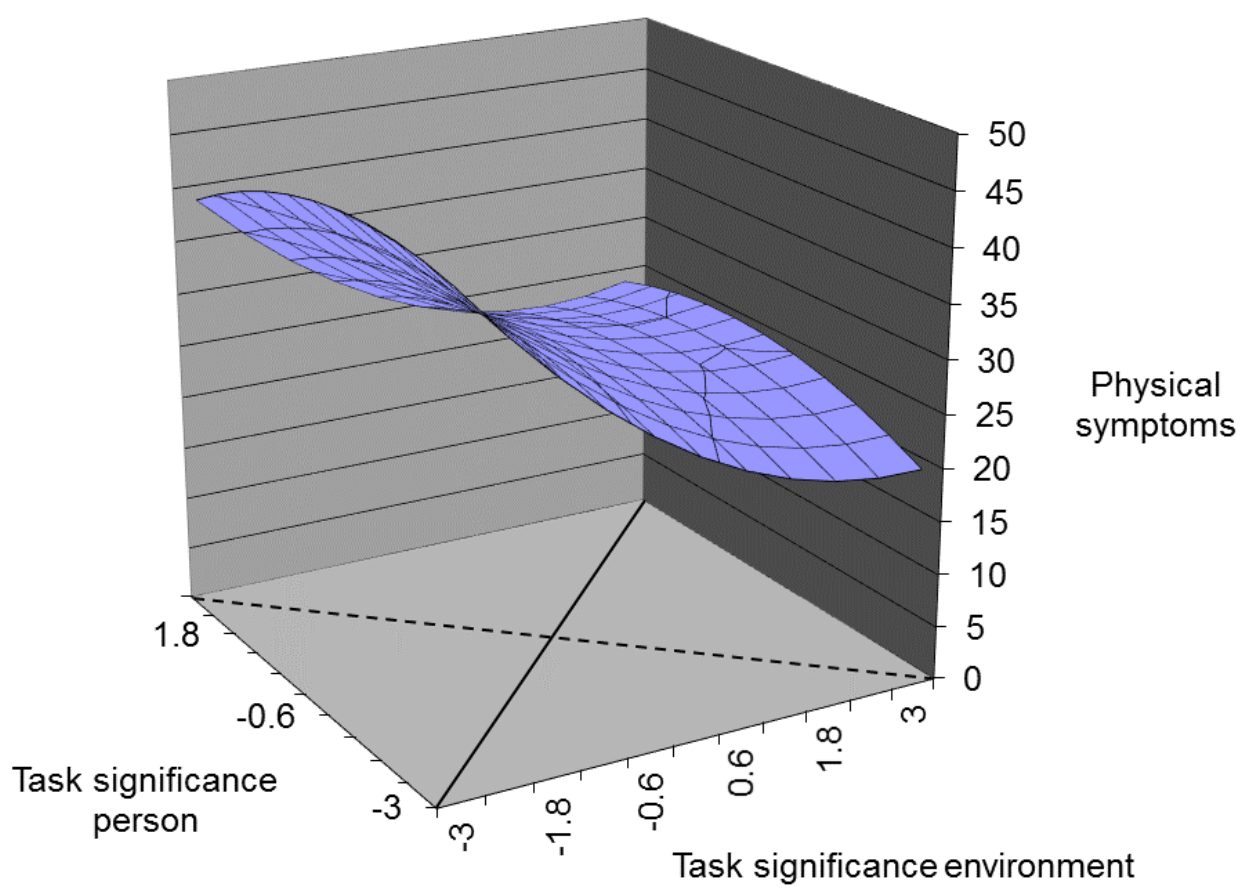

Figure 21. Task Significance Fit with Physical Symptoms. Response surface for task significance fit and outcome of physical symptoms. Solid line indicates line of fit; dashed line indicates line of misfit.

\section{Task significance to burnout relationship.}

Polynomial regression found a moderate effect for task significance fit with burnout $\left(R^{2}=.103\right)$ with all estimated regression coefficients as non-significant (Figure 22; Table A13).

\section{Nature of fit.}

Response surface analysis indicated a significant linear slope along the line of fit $\left(a_{1}=-0.241, p<.05\right)$ and a non-significant curvilinear effect $\left(a_{2}=-0.001, n s\right) . \mathrm{A}$ negative linear effect along the line of fit identifies a decreasing slope in which levels of burnout decrease along the line of fit from low preferred/present task significance to high preferred/present task significance. Specifically, less burnout was reported by those who 
had a high need for task significance that was being met by the nature of their job.

Comparatively, higher levels of burnout were reported by those who had a low need for task significance, even though this low need was also matched by low task significance present in their job. Note that, while partial contributions of regression coefficients were found not significant, the joint effects of these terms identified a significant effect of fit along the response surface.

\section{Nature of misfit.}

Results for task significance misfit indicated a non-significant slope and curvature of the surface along the line of misfit $\left(a_{3}=-0.050, n s ; a_{4}=0.090, n s\right)$. In other words, discrepancy between preferred and present task significance was not significantly related to levels of burnout.

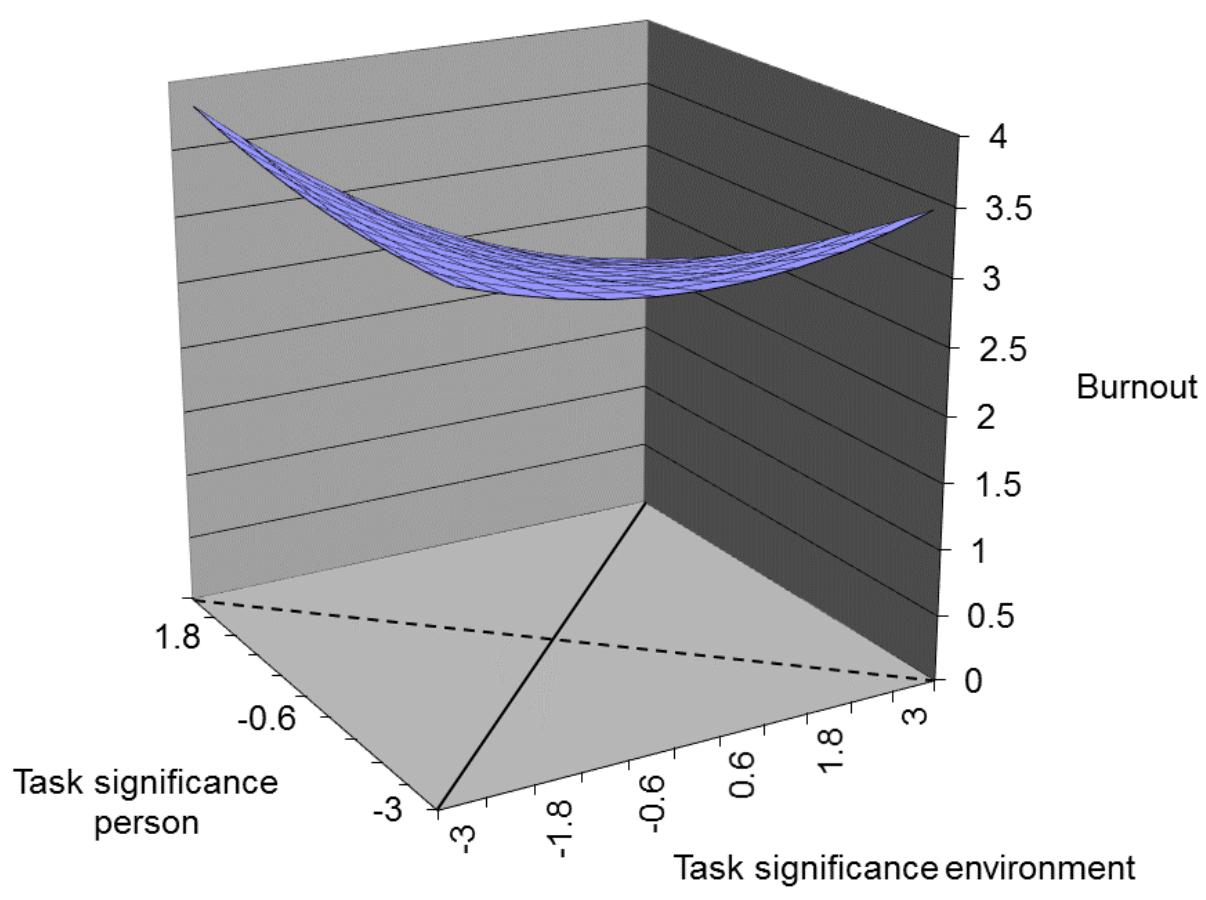

Figure 22. Task Significance Fit with Burnout. Response surface for task significance fit and outcome of burnout. Solid line indicates line of fit; dashed line indicates line of misfit. 


\section{Task significance to secondary traumatic stress relationship.}

Regression analysis of task significance fit with secondary traumatic stress found a small effect $\left(R^{2}=.031\right)$ with all estimated regression coefficients as non-significant (Figure 23; Table A14).

\section{Nature of fit.}

Response surface analysis found both slope and curvature along the line of fit as non-significant $\left(a_{1}=-0.173, n s ; a_{2}=0.025, n s\right)$. In respect to the outcome, this indicated no differences in one's experience of secondary traumatic stress along the line of fit, which represents agreement of preferred and present task significance. In other words, one's experience of secondary traumatic stress was not related to whether task significance fit was at low preferred/present, moderate preferred/present, or high preferred/present locations of agreement along the fit line continuum.

\section{Nature of misfit.}

Tests of linearity and curvilinearity of the line of misfit indicated non-significant linear and curvilinear effects $\left(a_{3}=-0.027, n s ; a_{4}=0.116, n s\right)$. In other words, discrepancy between preferred and present task significance was not significantly related to levels of secondary traumatic stress. Overall, results indicated task significance fit does not have a significant effect on secondary traumatic stress. 


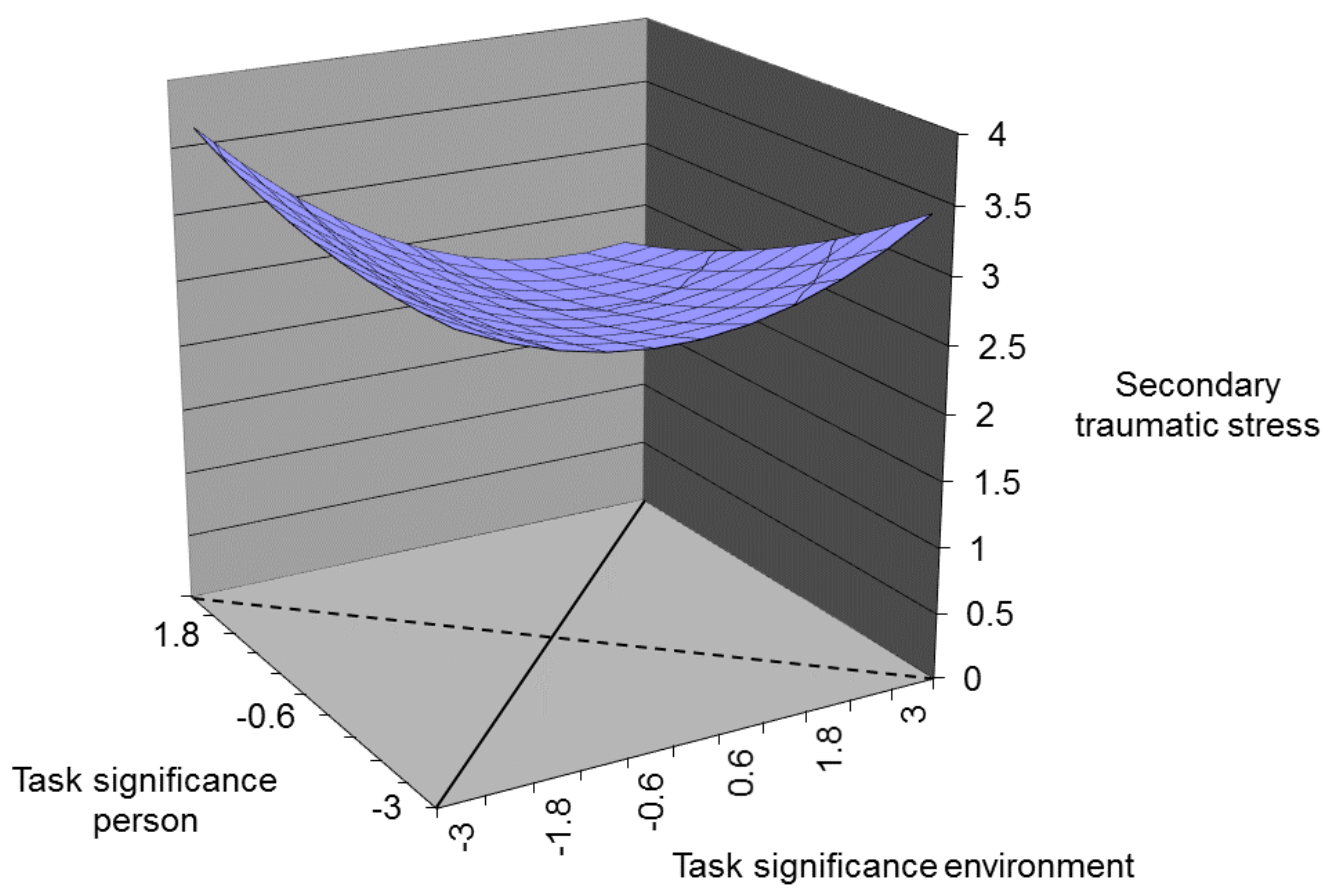

Figure 23. Task Significance Fit with Secondary Traumatic Stress. Response surface for task significance fit and secondary traumatic stress. Solid line indicates line of fit; dashed line indicates line of misfit.

\section{Task significance to compassion satisfaction relationship.}

Regression analysis found a large effect $\left(R^{2}=.244\right)$ for task significance fit with compassion satisfaction. Estimated regression coefficient for TsP was the only parameter found significantly predictive of compassion satisfaction (Figure 24; Table A15).

\section{Nature of fit.}

Results indicated a significant linear slope $\left(a_{1}=0.496, p<0.001\right)$ and a nonsignificant curvilinear effect $\left(a_{2}=-0.027, n s\right)$ along the line of fit. In respect to the outcome, a positive linear effect indicates an increase in compassion satisfaction along the line of fit - from low preferred/present task significance to high preferred/present task significance (Edwards, 2002; Shanock et al., 2010). In other words, compassion satisfaction was higher for emergency responders with high needs in task significance 
(that were being met by their job) compared to those with low needs in task significance (which were also being met by their job).

\section{Nature of misfit.}

Results for task significance misfit indicated non-significant slope and curvature of the surface along the line of misfit $\left(a_{3}=-0.027, n s ; a_{4}=-0.050, n s\right)$. In other words, discrepancy between preferred and present task significance was not significantly related to levels of compassion satisfaction.

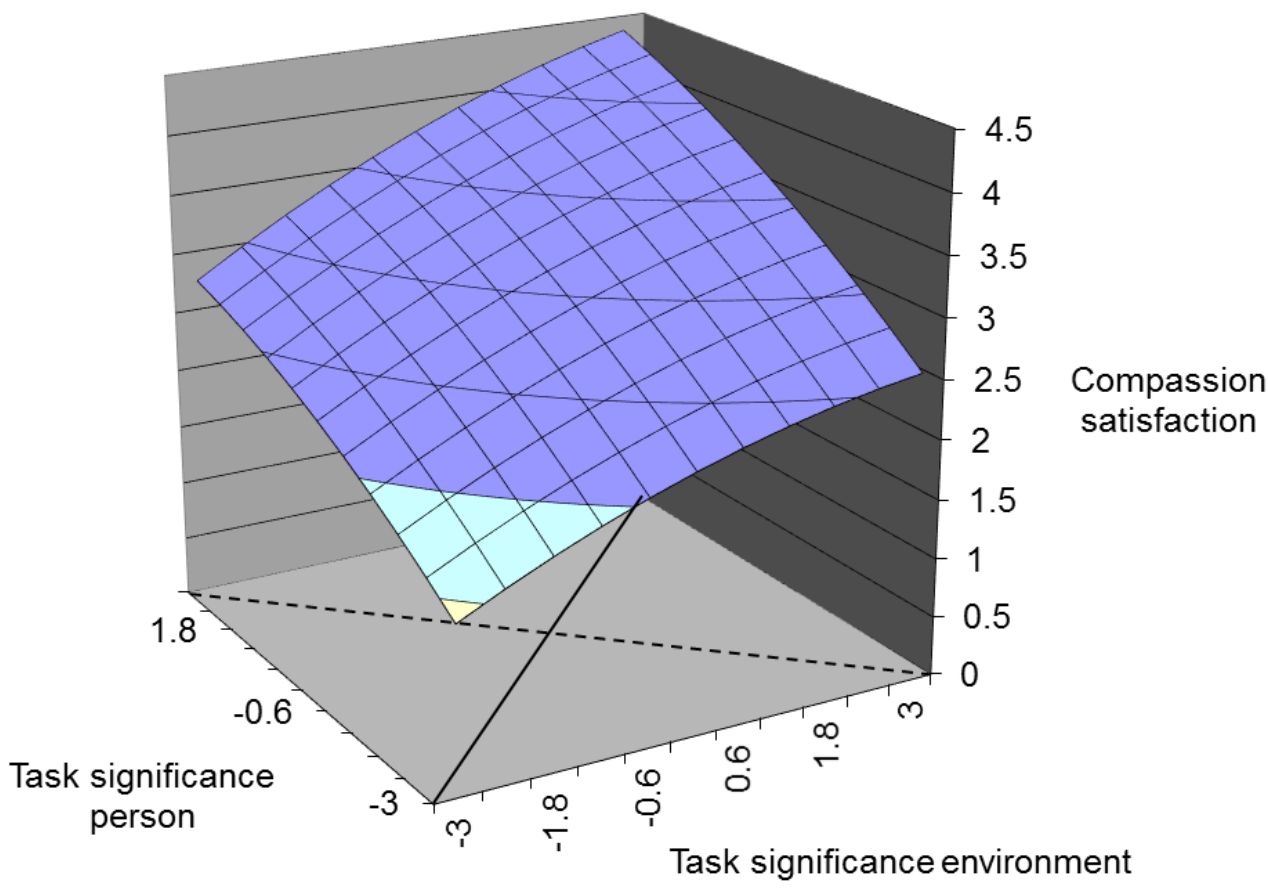

Figure 24. Task Significance Fit with Compassion Satisfaction. Response surface for task significance fit and compassion satisfaction. Solid line indicates line of fit; dashed line indicates line of misfit. 


\section{Autonomy}

Two constructs assessed participants' perception of autonomy fit. Autonomy environment (AuE) assessed how much autonomy employees perceived as present in their job; whereas autonomy person (AuP) indicated how much autonomy employees preferred in their job. On a 7-point scale with higher values indicating perception of more autonomy present, $\operatorname{AuE}\left(N=358, M=5.087, M_{s c}=1.09, S D=1.121, s^{2}=1.256\right)$ had a low reliability of .599 , with negative skew $(-0.724)$ and positive kurtosis $(0.393)$. Reliability for $\operatorname{AuP}\left(N=358, M=5.853, M_{s c}=1.85, S D=0.920, s^{2}=0.846\right)$ was .735 with a negative skew (-1.129) and positive kurtosis (2.201). Of note, AuE was significantly related to all outcomes, while AuP was not significantly related to any outcomes (see Table A3).

\section{Autonomy fit to physical symptoms relationship.}

Regression analysis of autonomy fit with physical symptoms found a small-tomoderate effect size $\left(R^{2}=.050\right)$. None of the estimated regression coefficients were significantly predictive of physical symptoms (Figure 25; Table A16).

\section{Nature of fit.}

Response surface analysis resulted in a non-significant linear slope $\left(a_{1}=-1.940\right.$, $n s)$ and a non-significant curvilinear effect $\left(a_{2}=0.169, n s\right)$ along the line of fit. In respect to the outcome, a non-significant slope indicates no significant relationship exists between frequency of physical symptoms and autonomy fit agreement along the fit line. In other words, as one moves along the line of fit from low preferred/present autonomy to high preferred/present autonomy, there were no differences in frequency of physical symptoms reported (Edwards, 2002; Shanock et al., 2010). 


\section{Nature of misfit.}

Similarly, results for autonomy misfit indicated a non-significant slope and curvature of the surface at the line of misfit $\left(a_{3}=1.242, n s ; a_{4}=1.105, n s\right)$. In other words, discrepancy between preferred and present autonomy was not significantly related to frequency of physical symptoms. Overall, results indicated autonomy fit does not have a significant effect of physical symptoms.

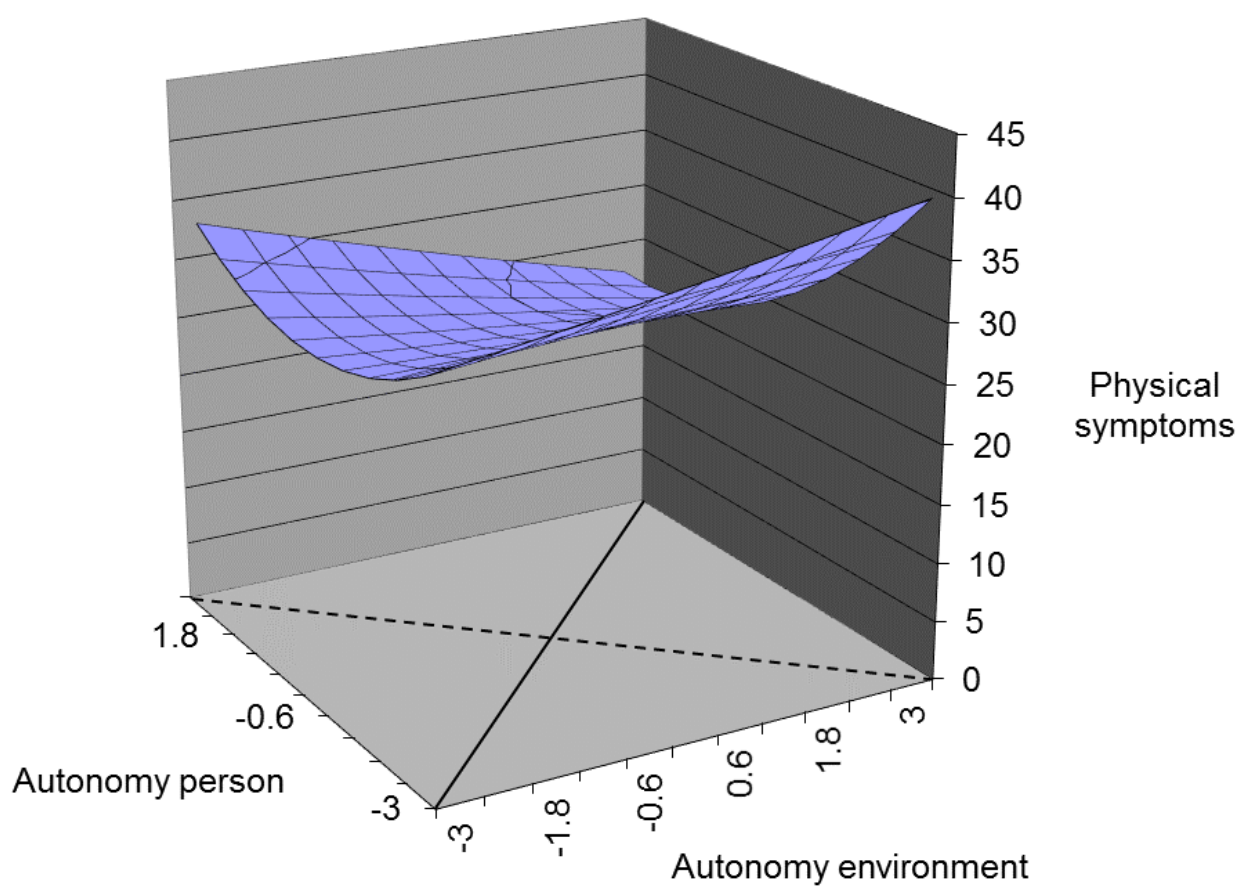

Figure 25. Autonomy Fit with Physical Symptoms. Response surface for autonomy fit and outcome of physical symptoms. Solid line indicates line of fit; dashed line indicates line of misfit.

\section{Autonomy fit to burnout relationship.}

Regression analysis of autonomy fit with burnout resulted in a moderate effect size $\left(R^{2}=.077\right)$ with estimated regression coefficient for $\mathrm{AuE}$ as the only significant predictor (Figure 26; Table A17). 


\section{Nature of fit.}

This analysis resulted in a non-significant linear slope $\left(a_{l}=-0.128, n s\right)$ and a nonsignificant curvilinear effect $\left(a_{2}=0.025, n s\right)$ along the line of fit. In respect to the outcome, a non-significant slope indicated no change in levels of burnout along the line of autonomy fit. Specifically, as one moves along the autonomy line of fit from low a preferred/present to high preferred/present, there were no differences in reported burnout levels (Edwards, 2002; Shanock et al., 2010).

\section{Nature of misfit.}

Results for autonomy misfit with burnout indicated non-significant slope and curvature of the surface at the line of misfit $\left(a_{3}=-0.195, n s ; a_{4}=0.026, n s\right)$. In other words, discrepancy between preferred and present autonomy was not significantly related to burnout. Overall, results indicated autonomy fit does not have a significant effect on burnout. 


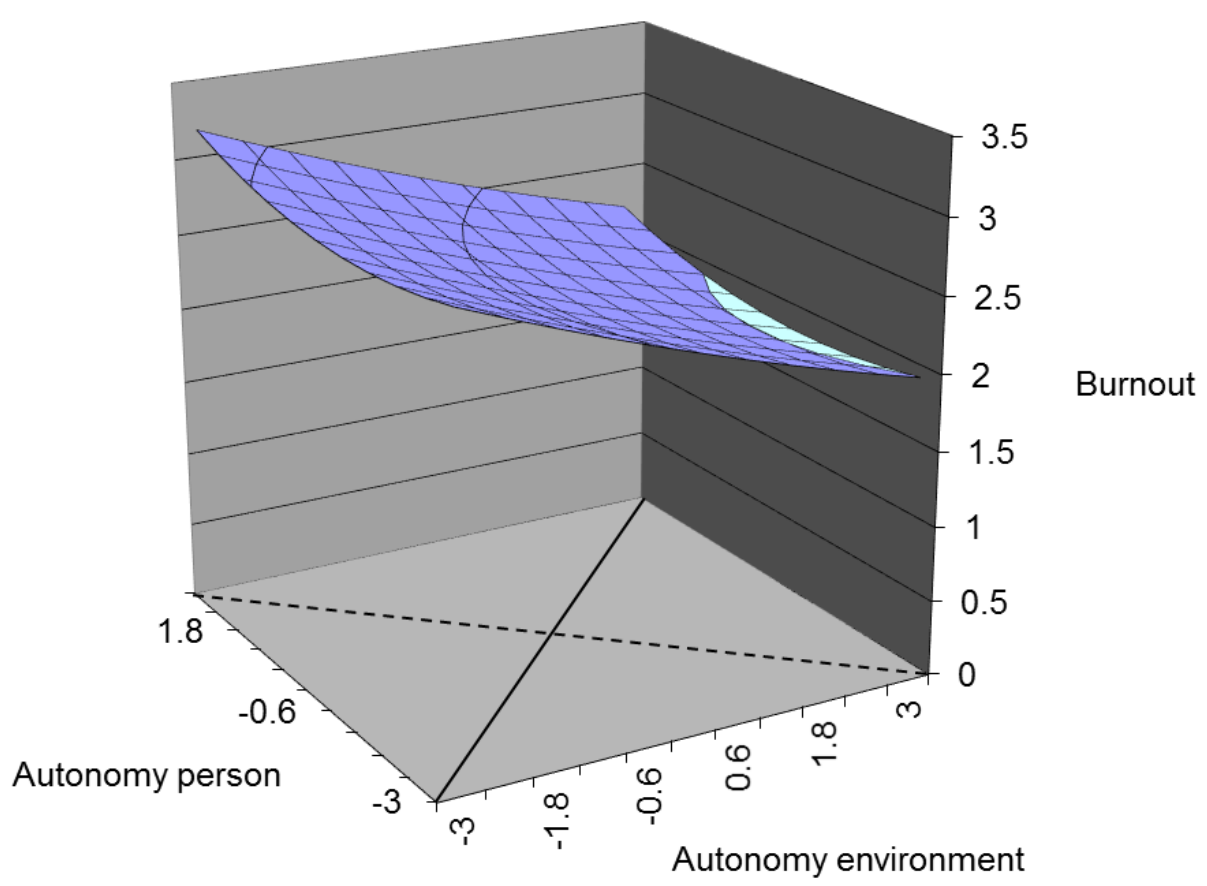

Figure 26. Autonomy Fit with Physical Symptoms. Response surface for autonomy fit and outcome of burnout. Solid line indicates line of fit; dashed line indicates line of misfit.

\section{Autonomy fit to secondary traumatic stress relationship.}

Regression results indicated a small effect for autonomy fit with secondary traumatic stress $\left(R^{2}=.032\right)$ with no significance found for any estimated regression coefficients (Figure 27; Table A18).

\section{Nature of fit.}

Response surface analysis resulted in a non-significant linear slope $\left(a_{1}=-0.034\right.$, $n s)$ and a non-significant curvilinear effect $\left(a_{2}=-0.005, n s\right)$ along the line of fit. In respect to the outcome, a non-significant slope indicated no significant changes in levels of secondary traumatic stress experienced along the autonomy line of fit. In other words, from low autonomy preferred/present to high autonomy preferred/present, there were no changes in experienced secondary traumatic stress (Edwards, 2002; Shanock et al., 2010). 


\section{Nature of misfit.}

To examine the nature of misfit in autonomy, as related to levels of secondary traumatic stress, similar tests of linearity and curvilinearity of the line of misfit were conducted. Response surface analysis of autonomy misfit indicated non-significant slope and curvature of the surface along the line of misfit $\left(a_{3}=-0.143, n s ; a_{4}=0.036, n s\right)$. In other words, discrepancy between preferred and present autonomy was not significantly related to secondary traumatic stress. Therefore, results indicated autonomy fit does not have a significant effect on secondary traumatic stress.

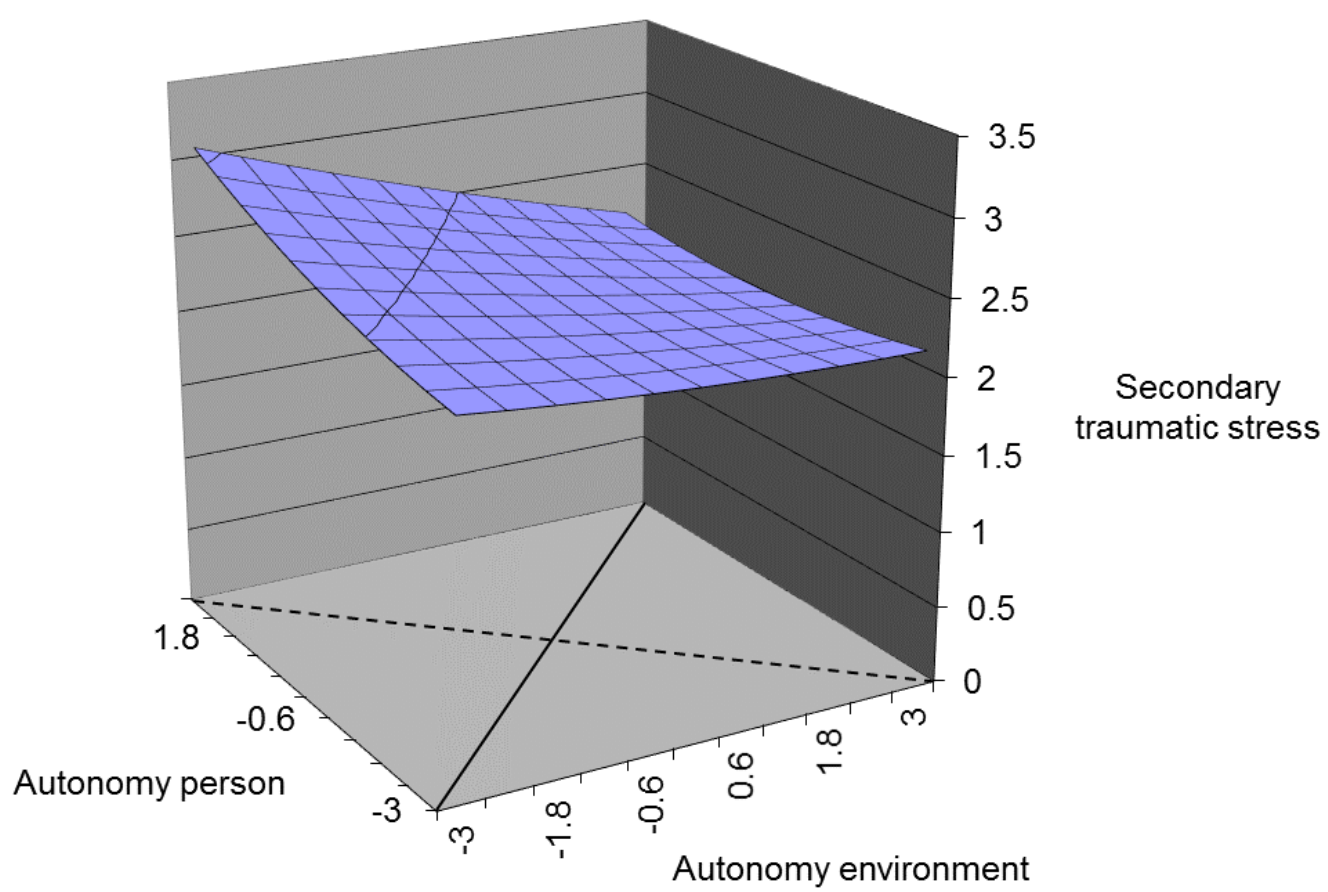

Figure 27. Autonomy Fit with Secondary Traumatic Stress. Response surface for autonomy fit and secondary traumatic stress. Solid line indicates line of fit; dashed line indicates line of misfit. 


\section{Autonomy fit to compassion satisfaction relationship.}

Polynomial regression results indicated a moderate effect $\left(R^{2}=.111\right)$ for autonomy fit with compassion satisfaction. Only the estimated regression coefficient for $\mathrm{AuE}$ was found significantly predictive of compassion satisfaction (Figure 28; Table A19).

\section{Nature of fit.}

Response surface analysis of autonomy fit with compassion satisfaction resulted in a non-significant linear slope $\left(a_{1}=0.205, n s\right)$ and a non-significant curvilinear effect $\left(a_{2}=-0.027, n s\right)$ along the line of fit. In respect to the outcome, a non-significant slope indicates no significant changes in levels of compassion satisfaction along the line of fit for autonomy. In other words, from low autonomy preferred/present to high autonomy preferred/present, there were no changes in compassion satisfaction levels (Edwards, 2002; Shanock et al., 2010).

\section{Nature of misfit.}

Results for autonomy misfit indicated non-significant slope and curvature of the surface at the line of misfit $\left(a_{3}=0.207, n s ; a_{4}=0.025, n s\right)$. In other words, discrepancy between preferred and present autonomy was not significantly related to the experience of compassion satisfaction. Therefore, results indicated autonomy fit does not have a significant effect on compassion satisfaction. 


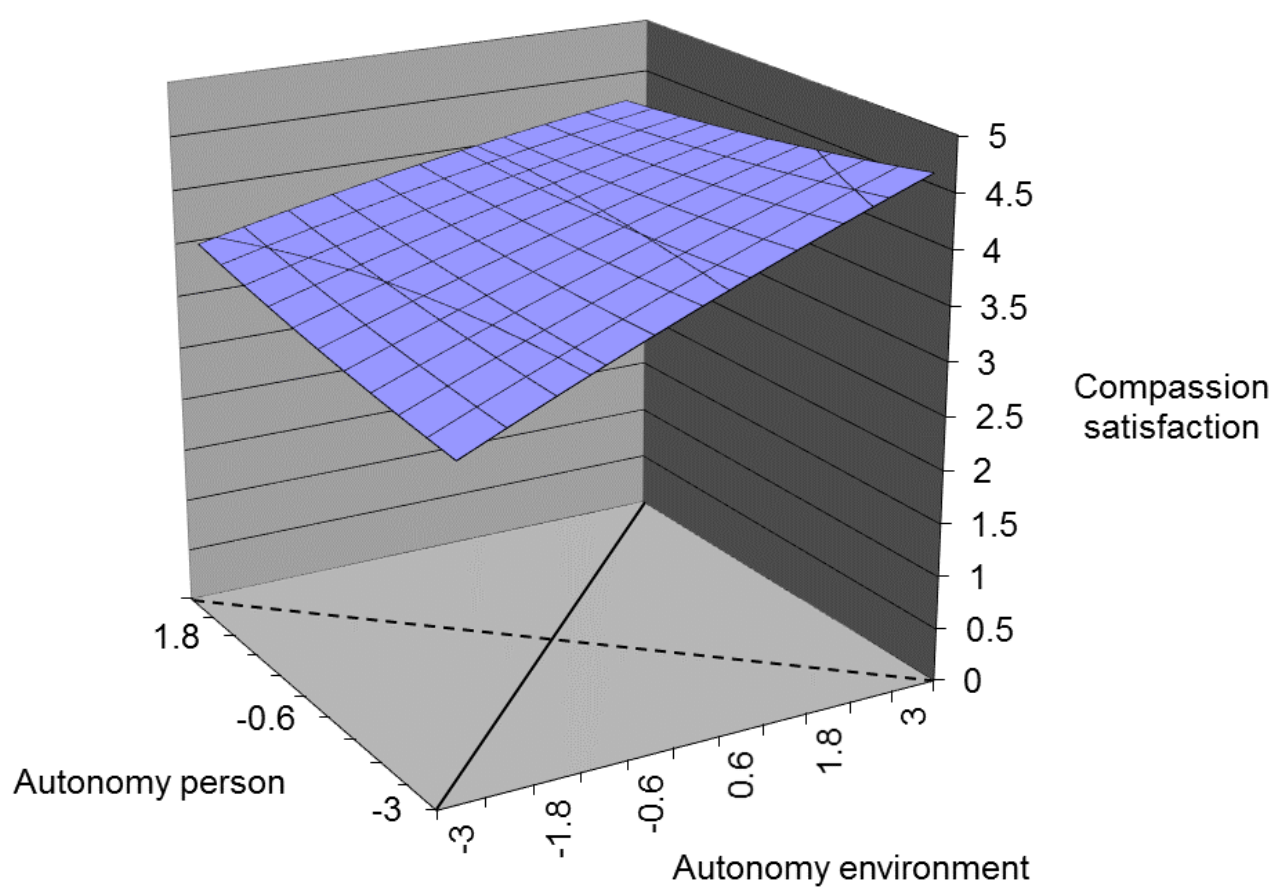

Figure 28. Autonomy Fit with Compassion Satisfaction. Response surface for autonomy fit and compassion satisfaction. Solid line indicates line of fit; dashed line indicates line of misfit.

\section{Feedback}

Feedback resulting from direct, clear information provided by the job itself was assessed in two components: how much job-based feedback employees perceived as present in their jobs (FjE) and how much job-based feedback was preferred (FjP). On a 7-point scale in which higher values indicated more job-based feedback present or preferred, FjE $\left(N=358, M=4.776, M_{s c}=0.78, S D=1.171, s^{2}=1.372\right)$ had an internal reliability of .699 , and relatively normal distribution $($ skew $=-0.426$, kurtosis $=0.002)$. FjP $\left(N=358, M=5.470, M_{s c}=1.47, S D=0.927, s^{2}=0.859\right)$ had an internal reliability of .738 , with a slight negative skew (-0.694) and more pronounced kurtosis (1.057). With the exception of the non-significant relationship between FjP and secondary traumatic stress, all predictor terms were significantly related to outcome variables (see Table A3). 


\section{Feedback fit to physical symptoms relationship.}

Polynomial regression results for feedback fit with physical symptoms found a moderate effect size $\left(R^{2}=.071\right)$. However, only the estimated regression coefficient for FjE was found significantly predictive of physical symptoms (Figure 29; Table A20).

\section{Nature of fit.}

Response surface analysis resulted in a significant linear slope $\left(a_{1}=-3.807, p<\right.$ $0.01)$ and a non-significant curvilinear effect $\left(a_{2}=0.721, n s\right)$ along the line of fit. In respect to the outcome, a negative linear effect along the line of fit indicates a decreasing slope along the line of fit. In other words, physical symptoms decrease an one moves along the line of fit from low preferred/present feedback from the job to high preferred/present feedback from the job (Edwards, 2002; Shanock et al., 2010).

\section{Nature of misfit.}

Results for feedback misfit indicated non-significant slope and curvature of the surface at the line of misfit $\left(a_{3}=-0.869, n s ; a_{4}=0.041, n s\right)$. In other words, discrepancy between preferred and present feedback from the job was not significantly related to the frequency of physical symptoms. 


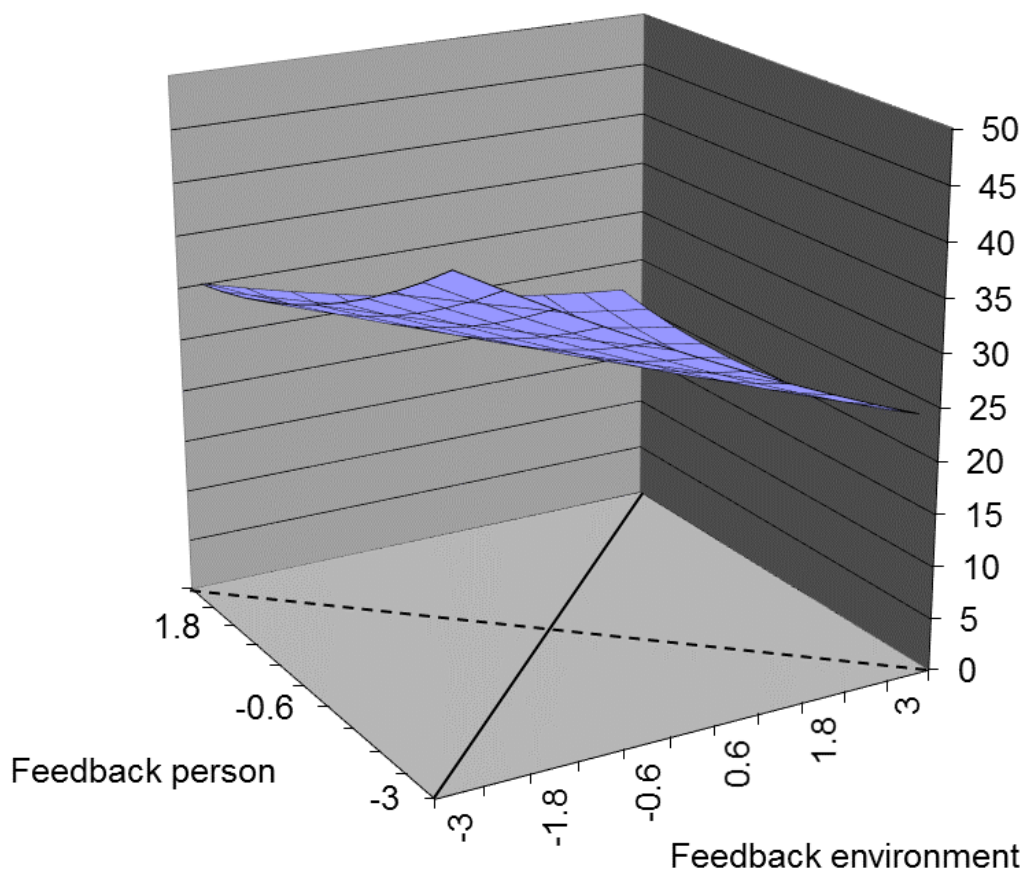

Physical symptoms

Figure 29. Feedback Fit with Physical Symptoms. Response surface for feedback fit and the outcome physical symptoms. Solid line indicates line of fit; dashed line indicates line of misfit.

\section{Feedback fit to burnout relationship.}

Regression analysis of feedback fit with burnout found a moderate-to-strong effect $\left(R^{2}=.176\right)$. However, only the estimated regression coefficient for FjE was found significantly predictive of burnout (Figure 30; Table A21).

\section{Nature of fit.}

This analysis resulted in a significant negative linear slope $\left(a_{1}=-0.323, p<0.01\right)$ and a non-significant curvilinear effect $\left(a_{2}=0.014, n s\right)$ along the line of fit. In respect to the outcome, a negative linear effect reflects decreasing burnout levels along the line of fit. In other words, burnout decreases as one moves along the fit line continuum from low preferred/present job-based feedback to high preferred/present job-based feedback (Edwards, 2002; Shanock et al., 2010). This finding implies that emergency responders 
whose jobs provide for their high needs in job-based feedback experience less burnout than those whose jobs provide for their low needs in job-based feedback. Even though both high needs and low needs were perceived as matched by their work environment, burnout was comparatively lower for emergency responders with high needs in job-based feedback.

\section{Nature of misfit.}

The nature of misfit in feedback, as related to levels of burnout, resulted in a significant slope $\left(a_{3}=-0.234, p<0.05\right)$ along the line of misfit and a non-significant curvilinear effect $\left(a_{4}=-0.084, n s\right)$. As previously discussed, a significant slope of the surface along the line of misfit indicates how the direction of discrepancy is related to the outcome (Shanock et al, 2010). In the case of the feedback fit-burnout surface, this significant negative slope indicated higher burnout levels when preferred feedback was greater than feedback present in the job. In other words, emergency responders reported higher levels of burnout when they desired more feedback than received. In comparison, emergency responders who received more feedback in the job than desired reported lower burnout levels.

Overall results indicated a complex relationship between job-based feedback fit and one's experience of burnout. Specifically, burnout levels were lower for emergency responders desiring a lot of job-based feedback. When there was a discrepancy between how much feedback was desired and how much was provided, those with more feedback than desired experienced lower burnout levels. 


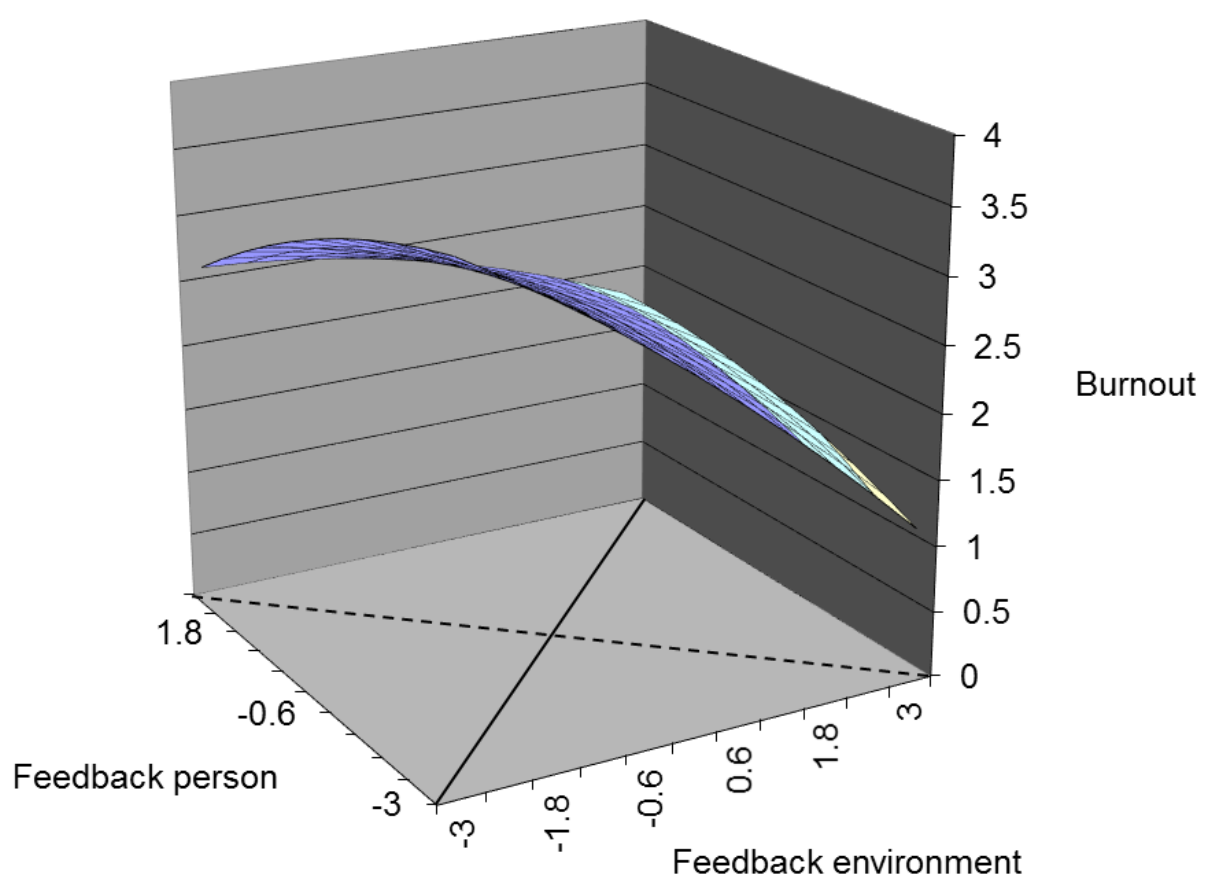

Figure 30. Feedback Fit with Burnout. Response surface for feedback fit and the outcome burnout. Solid line indicates line of fit; dashed line indicates line of misfit.

\section{Feedback fit to secondary traumatic stress relationship.}

Polynomial regression analysis of the feedback fit-secondary traumatic stress relationship found a moderate effect $\left(R^{2}=.072\right)$. However, only the estimated regression coefficient for FjE was found significantly predictive of secondary traumatic stress (Figure 31; Table A22).

\section{Nature of fit.}

This analysis resulted in a marginally significant linear slope $\left(a_{1}=-0.175, p=\right.$ $.055)$ and a non-significant curvilinear effect $\left(a_{2}=0.002, n s\right)$ along the line of fit. While in the direction expected such that secondary traumatic stress is reduced along the line of fit, this negative slope did not reach significance to indicate relative differences in 
secondary traumatic stress at low preferred/present feedback fit compared to high preferred/present feedback fit.

\section{Nature of misfit.}

Results of the response surface for feedback misfit also indicated a marginally significant slope and non-significant curvature of the surface along the line of misfit $\left(a_{3}=\right.$ $\left.-0.198, p=.058 ; a_{4}=-0.030, n s\right)$. While this indicated a trend for higher levels of secondary traumatic stress when one's preference for feedback exceeded the perception of amount of feedback provided, this linear effect did not reach a priori determined significance level.

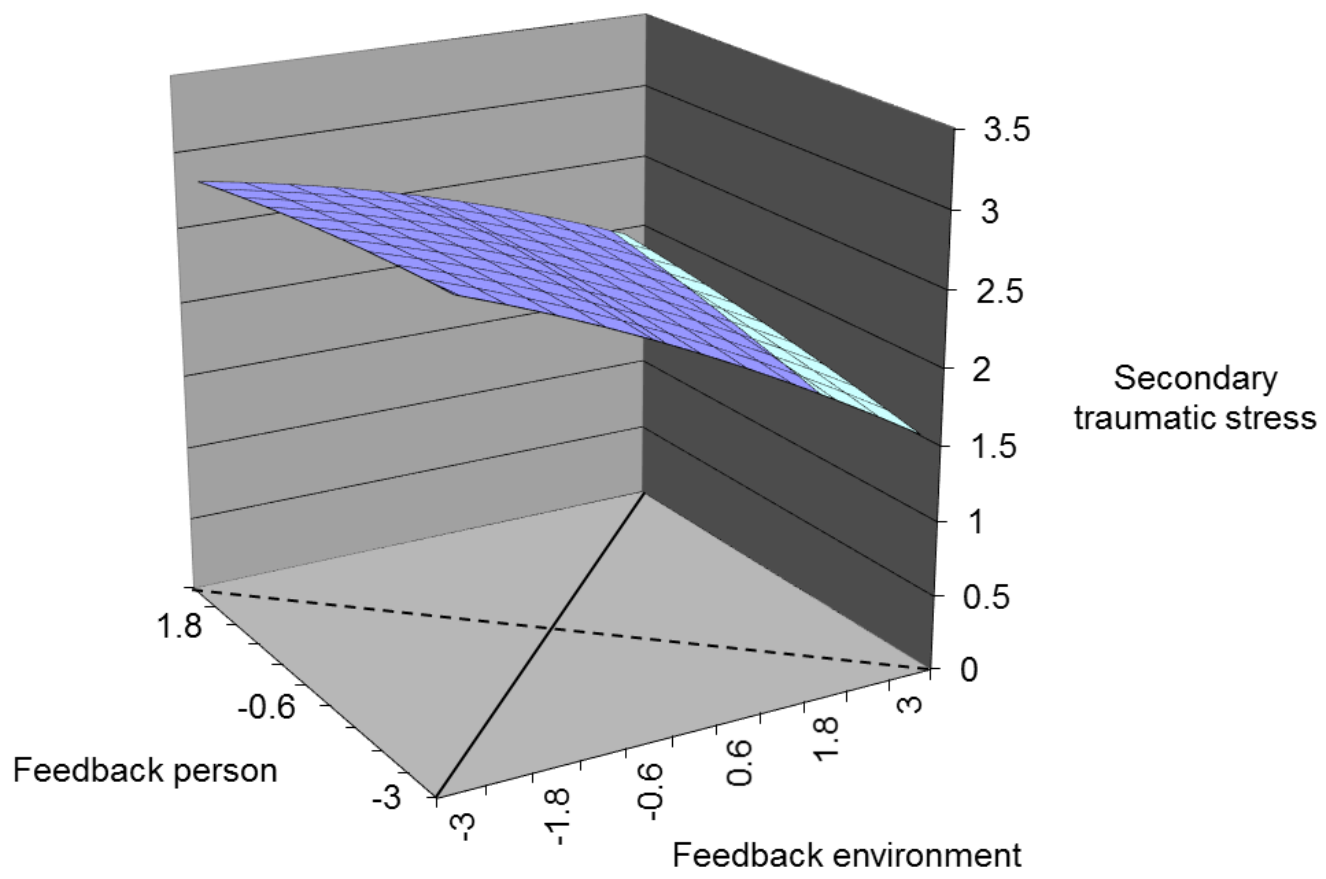

Figure 31. Feedback Fit with Secondary Traumatic Stress. Response surface for feedback fit and secondary traumatic stress. Solid line indicates line of fit; dashed line indicates line of misfit. 


\section{Feedback fit to compassion satisfaction relationship.}

Polynomial regression analysis of feedback fit with compassion satisfaction found a moderate-to-strong effect $\left(R^{2}=.182\right)$ with the estimated regression coefficient for FjE as the only significant predictor (Figure 32; Table A23).

\section{Nature of fit.}

Response surface analysis resulted in a significant positive linear slope along the line of fit $\left(a_{1}=0.391, p<.01\right)$, but a non-significant curvilinear effect $\left(a_{2}=-0.032, n s\right)$. In respect to the outcome, a positive slope indicates increases in compassion satisfaction along the feedback fit line. In other words, compassion satisfaction increased as one moves along the feedback line of fit from low preferred/present to high preferred/present (Edwards, 2002; Shanock et al., 2010). This finding suggests that emergency responders who were provided with the high amount of feedback they desired experienced higher levels of compassion satisfaction compared to those who desired a low amount of feedback - even though they perceived this low amount of feedback was matched by their job.

\section{Nature of misfit.}

The response surface along the line of misfit found non-significant slope and curvature $\left(a_{3}=0.155, n s ; a_{4}=0.087, n s\right)$. In other words, discrepancy between preferred and present feedback was not significantly related to levels of compassion satisfaction. 


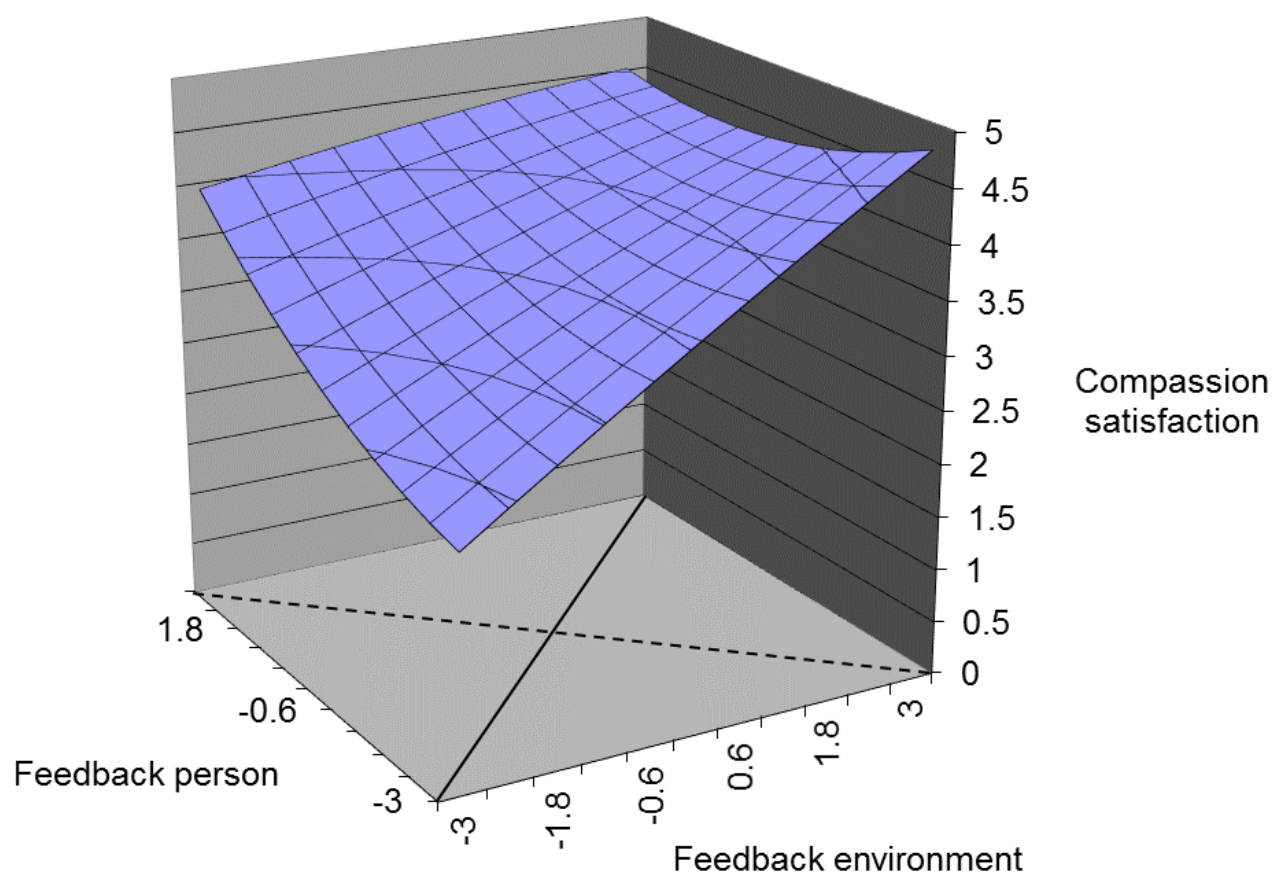

Figure 32. Feedback Fit with Compassion Satisfaction. Response surface for feedback fit and compassion satisfaction. Solid line indicates line of fit; dashed line indicates line of misfit.

\section{Summary of Results}

Across the five job characteristics dimensions, different effects were identified using polynomial regression and response surface analysis. Skill variety fit was only related to one outcome, compassion satisfaction. In relative comparison, compassion satisfaction was higher for emergency responders with high needs in skill variety that were being met by the job, compared to emergency responders with low needs in skill variety (that were also being met by the job). In terms of skill variety misfit, excess in skill variety in the job was not related to strain or well-being outcomes. In other words, discrepancy such that more skill variety was perceived as present in the job compared to preferred had no influence in either reduced or increased outcomes, suggesting an asymptotic misfit relationship for skill variety fit. As discussed in Chapter II, as supplies 
increase to meet one's needs strain is reduced. Once supplies have surpassed one's needs, excess in supplies may be related to increased strain (i.e., parabolic), reduced strain (i.e., monotonic), or have no effects on strain (i.e., asymptotic). This asymptotic relationship indicates that increases in skill variety past one's preferred amount does not contribute to reduced strain.

Task identity had significant relationships with two outcomes, burnout and secondary traumatic stress. In cases when one's preference for task identity was comparable to perceived task identity present in the job, burnout was relatively higher for emergency responders with moderate needs and supplies. In other words, emergency responders who indicated high preferred/present or low preferred/present in task identity fit reported significantly lower levels of burnout compared to emergency responders at the midpoint of task identity fit - even though their preference was also matched by the job's supply of that attribute. This finding is opposite to the curvilinear effect found by Xie and Johns (1995) in which burnout levels were lower for those at the midpoint of perceived task identity present in their jobs.

Misfit in task identity was related to higher levels of secondary traumatic stress for emergency responders. Specifically, higher levels of secondary traumatic stress were reported by emergency responders whose preference for task identity was not met by task identity provided in the job. This is consistent with theoretical forms of misfit established in PE fit theory such that strain is reduced as supplies increase to meet one's needs (Caplan et al., 1980; French et al., 1982; Harrison, 1978). Continued reduction in strain due to supplied task identity that exceeded one's needs indicated a monotonic form 
of excess in which employees might use oversupply to carryover or conserve for later (Edwards \& Shipp, 2007).

Task significance fit was related to outcomes of compassion satisfaction and burnout. In cases when one's preference for task significance was comparable to perceived task significance present in the job, there was a comparative difference in compassion satisfaction and burnout outcomes for emergency responders. Specifically, compared to their counterparts reporting low preferred/present task significance fit, emergency responders whose preference for and perceived presence of task significance were in agreement at a high preferred/presence location on the line of fit reported more compassion satisfaction. Also, emergency responders reporting high preferred/presence task significance fit reported less burnout, compared to their counterparts reporting low preferred/present task significance fit.

Surprisingly, autonomy fit was not related to any strain or well-being outcomes. This lack of significant effect was unexpected given the abundance of research supporting the importance of decision latitude and autonomy in the workplace.

Another surprising result was the relationship of job-based feedback fit to most strain and well-being outcomes. For the fit line, when job-based feedback provided by the job matched one's preference for feedback, emergency responders reporting high preferred/present feedback fit reported higher compassion satisfaction than their counterparts reporting low preferred/present feedback fit. Also, high preferred/present feedback fit was related to lower levels of burnout and physical symptoms compared to emergency responders reporting low preferred/present feedback fit. When there was a discrepancy or misfit between one's preference for job-based feedback and how much 
was perceived as present, higher burnout levels were reported by those whose feedback needs were not met by the job compared to emergency responders reporting more feedback present than they preferred. This represented a monotonic form of excess in which supplies that exceed needs might be used in other areas and thereby reduce strain (Edwards \& Shipp, 2007). 


\section{CHAPTER V: DISCUSSION}

\section{Summary of Results and Contributions}

This dissertation used updated analysis techniques to identify the nature of fit and misfit across specific job characteristics in relation to strain and/or well-being for emergency responders. Using PE fit as the primary theoretical framework, job characteristics were examined in terms of compatibility between one's preference for and perceived presence of each job characteristic. As a supplemental theoretical framework to PE fit theory, JCT by Hackman and Oldham $(1976,1980)$ provided job characteristics content dimensions of skill variety, task identity, task significance, autonomy and jobbased feedback. These job characteristics have a long history of use in work redesign and were selected with hopes that findings may provide a viable starting place of best opportunities for interventions towards work redesign efforts.

Using these job characteristics dimensions as person and environment predictor variables, fit (or misfit) was examined for its relationship to emergency responders' reports of strain and well-being outcomes. Strain and well-being outcomes selected for the dissertation represented areas found relevant to emergency responders: physical symptoms, burnout, secondary traumatic stress, and compassion satisfaction.

Two overarching research questions were examined in the dissertation:

1) What is the relationship with strain and well-being outcomes when one's preference for and perceived presence of a job attribute are matched?

2) When there is a discrepancy between one's preference for and perceived presence of a job attribute, does this discrepancy relate to strain and wellbeing outcomes? 
This section briefly summarizes findings and implications associated with the nature of fit and the nature of misfit in the context of the dissertation's research questions. Limitations and future research opportunities conclude Chapter V.

\section{Results and Implications Regarding the Nature of Fit}

As detailed throughout the dissertation, PE fit refers to the compatibility between a person and some aspect of their environment. The examination of PE fit in the context of this study examined person-job fit characterized by one's preference for and perceived presence of a specific job characteristic. Fit was operationalized as a situation in which one's preference for a job characteristic matched one's perception of that job characteristic as being present in one's job. This alignment or congruence of preference and presence could occur at any location along the line of fit. In other words, one's fit at low preferred/low present, moderate preferred/moderate present, or high preferred/high present was still considered fit. However, findings indicated relative differences in outcome levels associated with different locations along the line of fit. Examination of differential effects along the line of fit answered the first research question of the dissertation.

Fit in four job characteristics dimensions was related to differences in strain or well-being outcomes: skill variety fit, task identity fit, task significance fit, and job-based feedback fit. Specifically, the fit-outcome relationship of skill variety fit-compassion satisfaction indicated significantly higher compassion satisfaction levels for employees with high preference/high presence of skill variety fit, compared to those with low preference/low presence of skill variety fit. In other words, location of agreement along the line of fit mattered in one's experience of compassion satisfaction. 
A curvilinear effect was identified for the task identity fit-burnout relationship. Emergency responders reporting a match between their preference for task identity and the presence of task identity in the job reported higher levels of burnout when this alignment of preferred/present occurred at the middle point of the fit line. In other words, even though all points along the line of fit are matched, emergency responders with high preferred/present fit or low preferred/present fit reported less burnout than their counterparts reporting a moderate preferred/present fit. As noted previously, this curvilinear relationship is opposite to previous findings within a multiple industry sample conducted by Xie and Johns (1995). These contrasting results suggest a sample-based question. How does emergency responder work (or the subjective perception of their work) differ to affect the experience of burnout?

Task significance fit was found related to both burnout and compassion satisfaction. For the task significance-burnout relationship, there was a relative difference in burnout along the line of fit such that emergency responders reporting high preferred/present task significance fit reported less burnout compared to their counterparts reporting a low preferred/present task significance fit. Well-being as indicated by compassion satisfaction was also related to task significance fit. Specifically, emergency responders reporting a high preferred/present task significance fit also reported more compassion satisfaction compared to their counterparts reporting a low preferred/present task significance fit.

Similarly, job-based feedback fit was related to increase in well-being along the line of fit. Specifically, job-based feedback fit-compassion satisfaction relationship indicated higher levels of compassion satisfaction for emergency responders reporting a 
job-based feedback fit matched at high preferred/present fit compared to emergency responders matched at low preferred/present fit.

In terms of strain reduction, two fit-outcome relationships reported relative differences related to job-based feedback fit. Specifically, emergency responders reporting high preferred/present feedback fit reported less burnout and also reported less physical symptoms compared to their counterparts reporting low preferred/present feedback fit.

As fit indicates that employees perceive compatibility between what they want in their job and what is provided, why would any work redesign or change be helpful or relevant? Note that in significant fit-outcome relationships, alignment of preferred/present at a high location along the fit line was related to lowered strain and/or increased well-being. This is consistent with JCT's position that enriched jobs lead to beneficial organizational and personal outcomes (Hackman \& Oldham, 1976, 1980). The question then becomes, what impedes employees from desiring enriched jobs? While possibly a trait individual difference that is not affected by job redesign, some organizational factors (e.g., culture, climate, constraints) have been identified as barriers to employee needs satisfaction (Bakker \& Schaufeli, 2008; Johnson \& McIntye, 1998; Silverthorne, 2004; Xie \& Johns, 1995). Therefore, organizational initiatives to facilitate employee enrichment need to start with a comprehensive needs analysis of current organizational polices, practices, and characteristics. How these organizational factors affect employees' desire for enriched jobs may provide opportunities to take advantage of benefits found in high preferred/present fit. 


\section{Results and Implications Regarding the Nature of Misfit}

Misfit, discrepancy, or incompatibility between one's preference and their perceived presence of a job characteristic also had effects related to strain outcomes for emergency responders. Misfit occurs when an employee perceives more of a job attribute in their job than they wanted, but also when their preference for a job attribute is not provided for by the job. Examination of the nature of misfit and its relationship with outcomes answered the second question posed in the dissertation. When one's preferences were unmet by their job, effects of the misfit were identified for task identity and job-based feedback.

Task identity misfit was found related to secondary traumatic stress such that higher levels of secondary traumatic stress were reported by emergency responders whose preference for task identity was not met by task identity provided in the job. Specifically, task identity misfit was related to secondary traumatic stress such that high preference/low presence of task identity was significantly related to higher levels of secondary traumatic stress experienced by emergency responders. As previously defined, task identity refers to the extent one identifies with the results of their work efforts. Often, emergency responders are unaware of what happens to those they have helped once the event has passed. Results associated with task identity misfit suggest more opportunities to identify with how one's efforts affected final results for each incident may help to reduce secondary traumatic stress. Even if more task identification opportunities were provided than desired, the experience of secondary traumatic stress was lower compared to those who did not have their preferred amount of identification provided. 
This pattern of misfit was also found significant in the job-based feedback fitburnout relationship. Job-based feedback, which refers to clear information provided by the job itself, was posited to increase work motivation via knowledge of the effectiveness of one's performance (Hackman \& Oldham, 1976, 1980). When one's preference for feedback was not met by provision of feedback in the job, emergency responders reported higher levels of burnout. In other words, having too much information about one's performance was less detrimental (in terms of burnout experienced) compared to having too little information. Access to real-time status for emergency response events may provide this type of job-based feedback.

With the exception of these two job characteristics resulting in significant misfit relationships, all remaining fit-outcome relationships resulted in asymptotic forms of excess (Caplan et al., 1980; French et al., 1982; Harrison, 1978). In other words, discrepancy between one's preference and their perceived presence of skill variety, task significance, or autonomy had no relationship with strain or well-being for emergency responders.

Misfit is more responsive to work redesign efforts than fit because misfit identifies a deficiency (or excess) that is relevant for all employees. Whether employees perceive fit with their job or not, addressing an area in which more truly is better has overall impact for the whole organization. For the two significant misfit relationships identified, a perceived deficit in the organization's provision of the job characteristic was more detrimental than perceived oversupply. Specifically, providing opportunities for task identification and job-based feedback was generally better for all employees in terms of reduced secondary traumatic stress and burnout. This was true even if more task 
identity and feedback were supplied than employees desired. In other words, while the more is better assumption is not true for all characteristics (Harris \& Kacmar, 2006; Pierce \& Aguinis, 2013), it is true for attributes of task identity and job-based feedback for emergency responders.

Generally, organizations are moving towards a more holistic approach to facilitating employee well-being. Instead of focusing solely on the reduction of strain, they are also concerned with increased well-being for employees. This can be seen in wellness programs that offer health promotion activities such as meditation, yoga, or nature areas (Lohia, 2014; Mattke et al., 2013), or having leaders advocate creation of environments that support wellness (Allen, 2011). In terms of findings from the dissertation, skill variety fit was not found related to reduction in strain. However, its contribution to employee wellness was evident in its relationship to increased well-being, indicated by the construct of compassion satisfaction. Facilitating emergency responders' experience of fulfillment from being part of a work that helps others (Stamm, 2002) may be influenced by taking note of employee preferences for skill variety, task significance, and job-based feedback.

\section{Limitations and Future Directions}

The ongoing goal of this dissertation is to facilitate continued research contributing to emergency responders' health, well-being, and performance. During the process of the dissertation, several limitations surfaced that provide opportunities towards future work in this area.

The first limitation identified was in range restriction of discrepancy values. As noted in Shanock et al. (2010), "If it turns out that very few participants have discrepant 
values ... the practical value of exploring how discrepancies affect an outcome variable would be small" (p. 547). Post-hoc analysis identified discrepancy greater than $1 S D$ between person and environment components occurring in approximately $30-35 \%$ of the data across all job characteristics (see Table A24). While a cut-off to identify if sufficient discrepant values exist has not been established, Shanock and colleagues (2010) used a sample with approximately $45 \%$ discrepant data. Of note, the two job characteristics dimensions with the lowest percentage of discrepant values (skill variety and autonomy, both at 29.9\%) had the least amount of significant results; whereas job-based feedback with the highest percentage of discrepant data (34.5\%) had several significant results. While there is still value in exploring fit-outcome relationships despite lack of discrepancy values, future study design might include those who have voluntarily left emergency services employment as a possible source of discrepancy between needs and supplies and how this misfit affects turnover.

A second limitation can be claimed for self-reported data. While subjective PE fit is a key factor influencing strain outcomes (Caplan et al., 1980; French et al., 1982; Harrison, 1978), potential for common method bias may attenuate relationships (Podsakoff et al., 2003) and should be accounted for in future research design. Similar to work completed by Edwards et al. (2006) in which different methods of measurement were compared, congruence research that measures objective and subjective components, in addition to person and environment components, provides opportunities to test the integrity of reported relationships (Kristof-Brown et al., 2005).

Low reliabilities of several job characteristics predictors represent an additional limitation. Specifically, skill variety environment, skill variety person, task identity 
environment, task significance environment, task significance person, autonomy environment, and feedback environment all reported internal reliability less than .70 (Table A3). This weakness in psychometric properties for the Job Characteristics Model (Hackman \& Oldham, 1976, 1980) has been noted in prior meta-analyses (Fried \& Ferris, 1987; Morgeson \& Humphrey, 2006). In continuation of this line of inquiry, the Work Design Questionnaire (WDQ) developed by Morgeson and Humphrey (2006) may be an interesting comparison within the same emergency responders' industry. In WDQ, work characteristics are conceptualized into motivational, social, and contextual categories. All five of Hackman and Oldham's $(1976,1980)$ core job characteristics dimensions were included in Morgeson and Humphrey's (2006) expanded framework within the motivational category and subsequent research has used WDQ to measure work characteristics.

Significant associations between strain outcomes presents an opportunity to examine the impact of fit for generalized strain. Specifically, strain outcomes were found to have moderately high correlations $(r=.61$, physical symptoms and burnout; $r=.51$, physical symptoms and secondary traumatic stress; $r=.69$ burnout and secondary traumatic stress; see Table A3). These correlations suggest a composite or latent variable approach may reveal relationships undetected by regression modeling. For example, strain outcomes may reflect an unobserved latent variable of generalized strain. As structural equation modeling accounts for error variance, a cleaner picture of effects between person-job fit and strain outcomes may be accessible using a latent strain variable. 
Finally, does the fact that one is experiencing a strain outcome influence their preference for and/or their perception of a job characteristic? With cross-sectional data, direction of influence cannot be identified, and person-job fit cannot be confirmed as the driver in fit-outcome relationships. For example, if employees report high levels of compassion satisfaction, does this job attitude drive their preference for skill variety? Or, based on need fulfillment and core self-evaluation concepts, employees might seek opportunities to meet their needs for feelings of competence by pursuing opportunities to utilize their skills (Judge, Locke, Durham, \& Kluger, 1998). And a final example, for employees experiencing burnout, this may influence employees' desire for less task significance and perception of less task significance in their jobs, as they are already experiencing depersonalization (Maslach, 1982). This directional question regarding fitoutcome relationships would require longitudinal studies to identify causal effects. As previously mentioned, addressing these potential barriers to employee engagement in enriched jobs may also leverage beneficial outcomes indicated along the line of fit.

In addition to limitations noted, several findings from the dissertation encourage further questions of inquiry. For example, based on the disappointing findings in autonomy-fit, this job attribute should be reexamined across job industries more carefully. Is the expectation or definition of workplace autonomy similar for emergency responders, military, and para-militaristic situations - but different for other industries (e.g., organizationally flat structures)?

Of note, correlations between person and environment components of autonomy with outcomes indicated significant relationships for autonomy environment, but not for autonomy person (see Table A3). This suggests a relationship with outcomes not based 
in person-job fit but based in organizational practices supporting decision-making latitude. This pattern of significant relationships was also found for task identity, suggesting a stronger impact from organizational attributes of task identity than from personal preferences for task identity.

Based on the land-slide effects identified for job-based feedback fit, types and conditions of feedback provide a worthwhile direction to further examine feedback's impact on strain and well-being. Specifically, does agent-based feedback fit have similar impact on these outcomes? If not, what (qualitatively) is different between agent-based feedback and job-based feedback that explains differences in impact?

Going forward, plans are to examine covariates and moderators to the fit-outcome relationships identified. With the exception of employees' gender significantly related to physical symptoms $(r=.12)$, no demographic variables were related to strain or wellbeing outcomes. Therefore, covariates were not included for the dissertation's reported polynomial regression models. However, as shown in Table A3, tenure had significant relationships with job characteristics of autonomy $(r=.22)$ and feedback $(r=.15)$. Future research might consider the influence of longevity in position in terms of one's evolving fit with their jobs.

Two potential moderators that seem salient for emergency responders that were frequently identified in literature were traumatic exposure (e.g., How does perceived severity of exposure change fit-outcome relationships?) and the impact of support source (e.g., Does source of support - supervisor, coworkers, family, community, media - have differential effects on fit-outcome relationships?). Also, would individual differences in personality traits be related to high or low needs satisfaction across job characteristics? 
In other words, along the line of fit, which personality traits are most likely to benefit from a job providing high skill variety, task identity, task significance, autonomy, or jobbased feedback? These questions and future directions continue the dissertation's dedication to emergency responders' health and well-being. 


\section{REFERENCES}

Aiken, L. S., \& West, S. G. (1991). The effects of predictor scaling on coefficients of regression equations. In Multiple regression: Testing and interpreting interactions (pp. 28-48). Newbury Park, CA: Sage.

Alderfer, C. P. (1969). An empirical test of a new theory of human needs. Organizational Behavior and Human Performance, 4(2), 142-175. doi:10.1016/00305073(69)90004-X

Allen, J. (2011). Wellness leadership [white paper]. Retrieved from www.healthyculture.com

American Psychiatric Association. (2013). Trauma- and stressor-related disorders. In Diagnostic and statistical manual of mental disorders. American Psychiatric Association. doi:10.1176/appi.books.9780890425596.dsm07

Annett, J. (1969). Feedback and human behaviour. Victoria, Australia: Penguin Books Australia.

Bakker, A. B., \& Demerouti, E. (2007). The job demands resources model: State of the art. Journal of Managerial Psychology, 22(3), 309-328.

doi:10.1108/02683940710733115

Bakker, A. B., \& Schaufeli, W. B. (2008). Positive organizational behavior: engaged employees in flourishing organizations. Journal of Organizational Behavior, 29(2), 147-154. doi:10.1002/job.515

Baumeister, R. F., \& Leary, M. R. (1995). The need to belong: Desire for interpersonal attachments as a fundamental human motivation. Psychological Bulletin, 117(3), 497-529. doi:10.1037/0033-2909.117.3.497

Beehr, T. A., \& Newman, J. E. (1978). Job stress, employee health, and organizational effectiveness: A facet analysis, model, and literature review. Personnel Psychology, 31(3), 665-699. doi:10.1111/j.1744-6570.1978.tb02118.x

Belsley, D. A., Kuh, E., \& Welsch, R. E. (1980). Regression diagnostics: Identifying influential data and sources of collinearity. Hoboken, NJ: Wiley \& Sons. 
Bliese, P. D., Edwards, J. R., \& Sonnentag, S. (2017). Stress and well-being at work: A century of empirical trends reflecting theoretical and societal influences. Journal of Applied Psychology, 102(3), 389-402. doi:10.1037/ap10000109

Bowling, N. A., \& Hammond, G. D. (2008). A meta-analytic examination of the construct validity of the Michigan Organizational Assessment Questionnaire Job Satisfaction subscale. Journal of Vocational Behavior, 73(1), 63-77. doi:10.1016/j.jvb.2008.01.004

Box, G. E. P., \& Draper, N. R. (1987). Empirical model-building and response surfaces. New York, NY: Wiley.

Brown, T. M. (2004). Somatization. Medicine, 32(8), 34-35. doi:10.1383/medc.32.8.34.43177

Bruk-Lee, V., \& Spector, P. E. (2006). The social stressors-counterproductive work behaviors link: Are conflicts with supervisors and coworkers the same? Journal of Occupational Health Psychology, 11(2), 145-156. doi:10.1037/10768998.11.2.145

Caplan, R. D. (1987). Person-environment fit theory and organizations: Commensurate dimensions, time perspectives, and mechanisms. Journal of Vocational Behavior, 31(3), 248-267. doi:10.1016/0001-8791(87)90042-X

Caplan, R. D., Cobb, S., French, J. R. P., Harrison, R. V., \& Pinneau, S. R. (1980). Job demands and worker health: Main effects and occupational differences. Ann Arbor, MI: Institute for Social Research.

Champoux, J. E. (1980). A three sample test of some extensions to the Job Characteristics Model of work motivation. Academy of Management Journal, 23(3), 466-478. doi: $10.2307 / 255512$

Chilton, M. a, Hardgrave, B. C., \& Armstrong, D. J. (2005). Person-job cognitive style fit for software developers: The effect on strain and performance. Journal of Management Information Systems, 22(2), 193-226. doi:10.1080/07421222.2005.11045849

Chung-Yan, G. A. (2010). The nonlinear effects of job complexity and autonomy on job satisfaction, turnover, and psychological well-being. Journal of Occupational Health Psychology, 15(3), 237-251. doi:10.1037/a0019823 
Cohen, J. (1978). Partialed products are interactions: Partialed powers are curve components. Psychological Bulletin, 85(4), 858-866. doi:10.1037/00332909.85.4.858

Cohen, J. (1988). The analysis of variance. In Statistical power analysis for the behavioral sciences (2 ed., pp. 273-406). Hillsdale, NJ: Routledge.

Cohen, J., Cohen, P., West, S. G., \& Aiken, L. S. (2003). Applied multiple regression/correlation analysis for the behavioral sciences (3rd ed.). Routledge. doi:10.4324/9780203774441

Collins, P. A., \& Gibbs, A. C. C. (2003). Stress in police officers: A study of the origins, prevalence and severity of stress-related symptoms within a county police force. Occupational Medicine, 53(4), 256-264. doi:10.1093/occmed/kqg061

Cooper, C., Liukkonen, P., \& Cartwright, S. (1996). Stress prevention in the workplace: Assessing the costs and benefits to organisations. Dublin, Ireland: Luxembourg: Office for Official Publications of the European Communities.

Cronbach, L. J. (1958). Proposals leading to analytic treatment of social perception scores. In R. Tagiuri \& L. Petrullo (Eds.), Person perception and interpersonal behavior (pp. 353-379). Stanford, CA: Stanford University Press.

Cronbach, L. J. (1987). Statistical tests for moderator variables: Flaws in analyses recently proposed. Psychological Bulletin, 102(3), 414-417. doi:10.1037/00332909.102.3.414

Cummings, T. G., \& Cooper, C. L. (1979). A cybernetic framework for studying occupational stress. Human Relations, 32(5), 395-418. doi:10.1177/001872677903200504

Dawis, R. V., \& Lofquist, H. L. (1984). A psychological theory of work adjustment. Minneapolis, MN: University of Minnesota.

De Jonge, J., \& Schaufeli, W. B. (1998). Job characteristics and employee well-being: a test of Warr's Vitamin Model in health care workers using structural equation modelling. Journal of Organizational Behavior, 19(4), 387-407. doi:10.1002/(SICI)1099-1379(199807)19:4<387::AID-JOB851>3.0.CO;2-9 
Duxbury, L., \& Higgins, C. C. (2012). Caring for and about those who serve: Work-life conflict and employee well being within Canada's police. Retrieved from http://sprott.carleton.ca/directory/duxbury-linda/

Edwards, J. R. (1991). Person-job fit: A conceptual integration, and methodological critique. In C. L. Cooper \& I. T. Robertson (Eds.), International review of industrial and organizational psychology (Vol. 6, pp. 283-357). Oxford, England: John Wiley \& Sons.

Edwards, J. R. (1996). An examination of competing versions of the person-environment fit approach to stress. Academy of Management Journal, 39(2), 292-339. doi: $10.2307 / 256782$

Edwards, J. R. (2002). Alternatives to difference scores: Polynomial regression analysis and response surface methodology. In F. Drasgow \& N. Schmitt (Eds.), Measuring and analyzing behavior in organizations: Advances in measurement and data analysis (pp. 350-400). San Francisco, CA: Jossey-Bass.

Edwards, J. R. (2008). Person-environment fit in organizations: An assessment of theoretical progress. The Academy of Management Annals, 2(1), 167-230. doi:10.1080/19416520802211503

Edwards, J. R., Cable, D. M., Williamson, I. O., Lambert, L. S., \& Shipp, A. J. (2006). The phenomenology of fit: Linking the person and environment to the subjective experience of person-environment fit. Journal of Applied Psychology, 91(4), 802827. doi:10.1037/0021-9010.91.4.802

Edwards, J. R., Caplan, R. D., \& Harrison, R. V. (1998). Person-environment fit theory: Conceptual foundations, empirical evidence, and directions for future research. In C. L. Cooper (Ed.), Theories of organizational stress (pp. 28-67). Oxford: Oxford University Press.

Edwards, J. R., \& Harrison, R. V. (1993). Job demands and worker health: Threedimensional reexamination of the relationship between person-environment fit and strain. Journal of Applied Psychology, 78(4), 628-648. doi:10.1037/00219010.78.4.628

Edwards, J. R., \& Parry, M. E. (1993). On the use of polynomial regression equations as an alternative to difference scores in organizational research. Academy of Management Journal, 36(6), 1577-1613. doi:10.2307/256822 
Edwards, J. R., \& Rothbard, N. P. (1999). Work and Family Stress and Well-Being: An Examination of Person-Environment Fit in the Work and Family Domains. Organizational Behavior and Human Decision Processes, 77(2), 85-129. doi:10.1006/obhd.1998.2813

Edwards, J. R., \& Shipp, A. J. (2007). The relationship between person-environment fit and outcomes: An integrative theoretical framework. In C. Ostroff \& T. A. Judge (Eds.), Perspectives on organizational fit (pp. 209-258). New York, NY: Lawrence Erlbaum Associates.

Efron, B., \& Tibshirani, R. (1994). An introduction to the bootstrap. New York, NY: Chapman \& Hall.

Elder, C., Barkhuizen, G., Knoch, U., \& von Randow, J. (2007). Evaluating rater responses to an online training program for L2 writing assessment. Language Testing, 24(1), 37-64. doi:10.1177/0265532207071511

Enders, C. K. (2003). Using the expectation maximization algorithm to estimate coefficient alpha for scales with item-level missing data. Psychological Methods, 8(3), 322-337. doi:10.1037/1082-989X.8.3.322

Enders, C. K. (2010). Applied missing data analysis. New York, NY: Guilford Press.

Faul, F., Erdfelder, E., Lang, A.-G., \& Buchner, A. (2007). G*Power 3: A flexible statistical power analysis program for the social, behavioral, and biomedical sciences. Behavior Research Methods, 39(2), 175-191. doi:10.3758/BF03193146

Figley, C. R. (1999). Compassion fatigue: Toward a new understanding of the costs of caring. In B. H. Stamm (Ed.), Secondary traumatic stress: Self-care issues for clinicians, researchers, and educators (pp. 3-28). Baltimore, MD: The Sidran Press.

Fila, M. J., Purl, J., \& Griffeth, R. W. (2017). Job demands, control and support: Metaanalyzing moderator effects of gender, nationality, and occupation. Human Resource Management Review, 27(1), 39-60. doi:10.1016/j.hrmr.2016.09.004

French, J. R. P., Caplan, R. D., \& Harrison, R. V. (1982). The mechanisms of job stress and strain. Chichester, UK: Wiley. 
French, J. R. P., \& Kahn, R. L. (1962). A programmatic approach to studying the industrial environment and mental health. Journal of Social Issues, 18(3), 1-47. doi:10.1111/j.1540-4560.1962.tb00415.x

Fried, Y., \& Ferris, G. R. (1987). The validity of the job characteristics model: A review and meta-analysis. Personnel Psychology, 40(2), 287-322. doi:10.1111/j.17446570.1987.tb00605.x

Fried, Y., Laurence, G. A., Shirom, A., Melamed, S., Toker, S., Berliner, S., \& Shapira, I. (2013). The relationship between job enrichment and abdominal obesity: A longitudinal field study of apparently healthy individuals. Journal of Occupational Health Psychology, 18(4), 458-468. doi:10.1037/a0033730

Ganster, D. C., \& Rosen, C. C. (2013). Work stress and employee health. Journal of Management, 39(5), 1085-1122. doi:10.1177/0149206313475815

Garbarino, S., \& Magnavita, N. (2015). Work stress and metabolic syndrome in police officers: A prospective study. PLOS One, 10(12), e0144318. doi:10.1371/journal.pone.0144318

Gardner, D. G. (1986). Activation theory and task design: An empirical test of several new predictions. Journal of Applied Psychology, 71(3), 411-418. doi:10.1037/0021-9010.71.3.411

Gershon, R. R. M., Barocas, B., Canton, A. N., Li, X., \& Vlahov, D. (2009). Mental, physical, and behavioral outcomes associated with perceived work stress in police officers. Criminal Justice and Behavior, 36(3), 275-289. doi: $10.1177 / 0093854808330015$

Glover, P. A. (2000). "Feedback. I listened, reflected and utilized": Third year nursing students' perceptions and use of feedback in the clinical setting. International Journal of Nursing Practice. doi:10.1046/j.1440-172x.2000.00218.x

Graham, J. W. (2009). Missing data analysis: Making it work in the real world. Annual Review of Psychology, 60(1), 549-576. doi:10.1146/annurev.psych.58.110405.085530

Grant, A. M. (2008). The significance of task significance: Job performance effects, relational mechanisms, and boundary conditions. Journal of Applied Psychology, 93(1), 108-124. doi:10.1037/0021-9010.93.1.108 
Grant, A. M., Fried, Y., \& Juillerat, T. (2011). Work matters: Job design in classic and contemporary perspectives. In APA handbook of industrial and organizational psychology: Building and developing the organization. (Vol. 1, pp. 417-453). Washington, DC: American Psychological Association. doi:10.1037/12169-013

Greller, M. M., \& Parsons, C. K. (1992). Feedback and feedback inconsistency as sources of strain and self-evaluation. Human Relations, 45(6), 601-620. doi:10.1177/001872679204500604

Griffin, M. A., \& Clarke, S. (2011). Stress and well-being at work. In S. Zedeck (Ed.), APA handbook of industrial and organizational psychology: Maintaining, expanding, and contracting the organization. (Vol. 3, pp. 359-397). American Psychological Association. doi:10.1037/12171-010

Griffin, M. L., Hogan, N. L., \& Lambert, E. G. (2012). Doing “people work" in the prison setting. Criminal Justice and Behavior, 39(9), 1131-1147. doi:10.1177/0093854812442358

Grigsby, D. W., \& McKnew, M. A. (1988). Work-stress burnout among paramedics. Psychological Reports, 63(1), 55-64. doi:10.2466/pr0.1988.63.1.55

Hackman, J. R., \& Lawler, E. E. (1971). Employee reactions to job characteristics. Journal of Applied Psychology, 55(3), 259-286. doi:10.1037/h0031152

Hackman, J. R., \& Oldham, G. R. (1975). Development of the Job Diagnostic Survey. Journal of Applied Psychology, 60(2), 159-170. doi:10.1037/h0076546

Hackman, J. R., \& Oldham, G. R. (1976). Motivation through the design of work: Test of a theory. Organizational Behavior and Human Performance, 16(2), 250-279. doi:10.1016/0030-5073(76)90016-7

Hackman, J. R., \& Oldham, G. R. (1980). Work redesign. Reading, MA: AddisonWesley.

Harris, K. J., \& Kacmar, K. M. (2006). Too much of a good thing: The curvilinear effect of leader-member exchange on stress. The Journal of Social Psychology, 146(1), $65-84$.

Harrison, R. V. (1976). Job demands and worker health: Person-environment misfit (Doctoral dissertation, University of Michigan). Retrieved from http://psycnet.apa.org/record/1978-06809-001 
Harrison, R. V. (1978). Person-environment fit and job stress. In C. L. Cooper \& R. Payne (Eds.), Stress at work (pp. 23-55). New York, NY: John Wiley \& Sons.

Hart, P. M., \& Cooper, C. L. (2001). Occupational stress: Toward a more integrated framework. In N. Anderson, D. S. Ones, \& C. Viswesvaran (Eds.), Handbook of industrial, work and organizational psychology (Vol. 2, pp. 93-114). London, UK. doi:10.4135/9781848608368.n6

Häusser, J. A., Mojzisch, A., Niesel, M., \& Schulz-Hardt, S. (2010). Ten years on: A review of recent research on the Job Demand-Control (-Support) model and psychological well-being. Work and Stress, 24(1), 1-35. doi:10.1080/02678371003683747

Helgoe, L. (2010). Revenge of the introvert [Webpage]. Retrieved from https://www.psychologytoday.com/us/articles/201009/revenge-the-introvert

Hollenbeck, J. R. (1989). Control-theory and the perception of work environments: The effects of focus of attention on affective and behavioral reactions to work. Organizational Behavior and Human Decision Processes, 43(3), 406-430. doi:10.1016/0749-5978(89)90045-9

Hooper, C., Craig, J., Janvrin, D. R., Wetsel, M. A., \& Reimels, E. (2010). Compassion satisfaction, burnout, and compassion fatigue among emergency nurses compared with nurses in other selected inpatient specialties. Journal of Emergency Nursing, 36(5), 420-427. doi:10.1016/j.jen.2009.11.027

Huang, Y., Lee, J., McFadden, A. C., Rineer, J., \& Robertson, M. M. (2017). Individual employee's perceptions of "Group-level Safety Climate" (supervisor referenced) versus "Organization-level Safety Climate" (top management referenced): Associations with safety outcomes for lone workers. Accident Analysis and Prevention, 98, 37-45. doi:10.1016/j.aap.2016.09.016

Hulin, C. L., \& Blood, M. R. (1968). Job enlargement, individual differences, and worker responses. Psychological Bulletin, 69(1), 41-55. doi:10.1037/h0025356

Humphrey, S. E., Nahrgang, J. D., \& Morgeson, F. P. (2007). Integrating motivational, social, and contextual work design features: A meta-analytic summary and theoretical extension of the work design literature. Journal of Applied Psychology, 92(5), 1332-1356. doi:10.1037/0021-9010.92.5.1332 
Huyghebaert, T., Gillet, N., Lahiani, F. J., \& Fouquereau, E. (2016). Curvilinear effects of job characteristics on ill-being in the nursing profession: A cross-sectional study. Journal of Advanced Nursing, 72(5), 1109-1121. doi:10.1111/jan.12894

Idaszak, J. R., \& Drasgow, F. (1987). A revision of the Job Diagnostic Survey: Elimination of a measurement artifact. Journal of Applied Psychology, 72(1), 6974. doi:10.1037/0021-9010.72.1.69

Ilgen, D. R., \& Hollenbeck, J. R. (1991). The structure of work: Job design and roles. In M. D. Dunnette \& L. M. Hough (Eds.), Handbook of industrial and organizational psychology (pp. 165-207). Palo Alto, CA: Consulting Psychologists Press.

Johns, G. (1981). Difference score measures of organizational behavior variables: A critique. Organizational Behavior and Human Performance, 27(3), 443-463. doi:10.1016/0030-5073(81)90033-7

Johnson, J. J., \& McIntye, C. L. (1998). Organizational culture and climate correlates of job satisfaction. Psychological Reports, 82(3), 843-850. doi:10.2466/pr0.1998.82.3.843

Judd, C. M., McClelland, G. H., \& Culhane, S. E. (1995). Data analysis: Continuing issues in the everyday analysis of psychological data. Annual Review of Psychology, 46(1), 433-465. doi:10.1146/annurev.ps.46.020195.002245

Judge, T. A., \& Ferris, G. R. (1992). The elusive criterion of fit in human resources staffing decisions. Human Resource Planning, 15(4), 47-67.

Judge, T. A., Locke, E. A., Durham, C. C., \& Kluger, A. N. (1998). Dispositional effects on job and life satisfaction: The role of core evaluations. Journal of Applied Psychology, 83(1), 17-34. doi:10.1037/0021-9010.83.1.17

Kahn, R. L., \& Byosiere, P. (1992). Stress in organizations. In M. D. Dunnette \& L. M. Hough (Eds.), Handbook of industrial organizational psychology (pp. 571-650). Palo Alto, CA: Consulting Psychologists Press.

Karasek, R. A. (1979). Job demands, job decision latitude, and mental strain: Implications for job redesign. Administrative Science Quarterly, 24(2), 285. doi: $10.2307 / 2392498$ 
Karasek, R., \& Theorell, T. (1990). Healthy work: Stress, productivity, and the reconstruction of working life. New York, NY: Basic Books.

Kristof-Brown, A., \& Guay, R. P. (2011). Person-environment fit. In S. Zedeck (Ed.), APA handbook of industrial and organizational psychology: Maintaining, expanding, and contracting the organization. (Vol. 3, pp. 3-50). Washington, DC: American Psychological Association. doi:10.1037/12171-001

Kristof-Brown, A. L., Zimmerman, R. D., \& Johnson, E. C. (2005). Consequences of individuals' fit at work: A meta-analysis of person-job, person-organization, person-group, and person-supervisor fit. Personnel Psychology, 58(2), 281-342. doi:10.1111/j.1744-6570.2005.00672.x

Kulik, C. T., Oldham, G. R., \& Hackman, J. R. (1987). Work design as an approach to person-environment fit. Journal of Vocational Behavior, 31(3), 278-296. doi:10.1016/0001-8791(87)90044-3

Kulka, A. (1979). Interaction as person-environment fit. In L. R. Kahle (Ed.), Methods for studying person-situation interactions (pp. 55-71). San Francisco, CA: Jossey-Bass.

Lambert, E. G. (2004). The impact of job characteristics on correctional staff members. The Prison Journal, 84(2), 208-227. doi:10.1177/0032885504265078

Lambert, E. G., Hogan, N. L., Dial, K. C., Jiang, S., \& Khondaker, M. I. (2012). Is the job burning me out? An exploratory test of the Job Characteristics Model on the emotional burnout of prison staff. The Prison Journal, 92(1), 3-23. doi: $10.1177 / 0032885511428794$

Lewin, K. (1943). Defining the "field at a given time." Psychological Review, 50(3), 292-310. doi: $10.1037 / \mathrm{h} 0062738$

Little, R. J. A., \& Rubin, D. B. (1987). Statistical analysis with missing data. New York, NY: John Wiley \& Sons.

Locke, E. A. (1969). What is job satisfaction? Organizational Behavior and Human Performance, 4(4), 309-336. doi:10.1016/0030-5073(69)90013-0

Locke, E. A. (1976). The nature and causes of job satisfaction. In M. D. Dunnette (Ed.), Handbook of industrial and organizational psychology. Chicago, IL: Rand McNally. 
Lohia, A. (2014). Corporate yoga: Revive your wellness program [Webpage]. Retrieved from http://www.corporatewellnessmagazine.com/worksite-wellness/wellnessprogram/

Maslach, C. (1982). Burnout: The cost of caring. Englewood Cliffs, NJ: Prentice-Hall.

Maslach, C., \& Jackson, S. E. (1981). The measurement of experienced burnout. Journal of Organizational Behavior, 2(2), 99-113. doi:10.1002/job.4030020205

Maslow, A. H. (1943). A theory of human motivation. Psychological Review, 50(4), 370396. doi:10.1037/h0054346

Mattke, S., Liu, H., Caloyeras, J. P., Huang, C. Y., Busum, K. R. Van, Khodyakov, D., \& Shier, V. (2013). Workplace wellness programs study: Final report. RAND Health, 3(2), 1-165. doi:10.1214/07-EJS057

Morgeson, F. P., \& Humphrey, S. E. (2006). The Work Design Questionnaire (WDQ): Developing and validating a comprehensive measure for assessing job design and the nature of work. Journal of Applied Psychology, 91(6), 1321-1339. doi:10.1037/0021-9010.91.6.1321

Muchinsky, P. M., \& Monahan, C. J. (1987). What is person-environment congruence? Supplementary versus complementary models of fit. Journal of Vocational Behavior, 31(3), 268-277. doi:10.1016/0001-8791(87)90043-1

Muthén, L. K., \& Muthén, B. O. (2012). Mplus user's guide (7th ed.). Los Angeles, CA: Author. doi: 1558407

Newman, D. A. (2014). Missing data: Five practical guidelines. Organizational Research Methods, 17(4), 372-411. doi:10.1177/1094428114548590

Nunnally, J. (1962). The analysis of profile data. Psychological Bulletin, 59(4), 311-319. doi: $10.1037 / \mathrm{h} 0041246$

O’Brien, G. E. (1983). Skill-utilization, skill-variety and the Job Characteristics Model. Australian Journal of Psychology, 35(3), 461-468. doi:10.1080/00049538308258757

Pavot, W., \& Diener, E. (1993). Review of the Satisfaction with Life scale. Psychological Assessment, 5(2), 164-172. doi:10.1037/1040-3590.5.2.164 
Peterson, N. G., Mumford, M. D., Borman, W. C., Jeanneret, P. R., Fleishman, E. A., Levin, K. Y., ... Dye, D. M. (2001). Understanding work using the occupational information network $\left(\mathrm{O}^{*} \mathrm{NET}\right)$ : Implications for practice and research. Personnel Psychology, 54(2), 451-492. doi:10.1111/j.1744-6570.2001.tb00100.x

Pierce, J. R., \& Aguinis, H. (2013). The too-much-of-a-good-thing effect in management. Journal of Management, 39(2), 313-338. doi:10.1177/0149206311410060

Podsakoff, P. M., MacKenzie, S. B., Lee, J.-Y., \& Podsakoff, N. P. (2003). Common method biases in behavioral research: A critical review of the literature and recommended remedies. Journal of Applied Psychology, 88(5), 879-903. doi:10.1037/0021-9010.88.5.879

Porter, L. W. (1962). Job attitudes in management: Perceived deficiencies in need fulfillment as a function of job level. Journal of Applied Psychology, 46(6), 375384. doi: $10.1037 / \mathrm{h} 0047808$

Porter, S. R., Whitcomb, M. E., \& Weitzer, W. H. (2004). Multiple surveys of students and survey fatigue. New Directions for Institutional Research, 2004(121), 63-73. doi:10.1002/ir.101

Potter, C. (2006). To what extent do nurses and physicians working within the emergency department experience burnout: A review of the literature. Australasian Emergency Nursing Journal, 9(2), 57-64. doi:10.1016/j.aenj.2006.03.006

Regehr, C. (2009). Social support as a mediator of psychological distress in firefighters. The Irish Journal of Psychology, 30(1-2), 87-98. doi:10.1080/03033910.2009.10446300

Regehr, C., Goldberg, G., \& Hughes, J. (2002). Exposure to human tragedy, empathy, and trauma in ambulance paramedics. American Journal of Orthopsychiatry, 72(4), 505-513. doi:10.1037/0002-9432.72.4.505

Rupprecht, E. A., Kueny, C. R., Shoss, M. K., \& Metzger, A. J. (2016). Getting what you want: How fit between desired and received leader sensitivity influences emotion and counterproductive work behavior. Journal of Occupational Health Psychology, 21(4), 443-454. doi:10.1037/a0040074

Ryan, R., \& Deci, E. (2000). Self-determination theory and the facilitation of intrinsic motivation. American Psychologist, 55(1), 68-78. doi:10.1037/0003066X.55.1.68 
Saks, A. M., \& Ashforth, B. E. (1997). A longitudinal investigation of the relationships between job information sources, applicant perceptions of fit, and work outcomes. Personnel Psychology, 50(2), 395-426. doi:10.1111/j.1744-6570.1997.tb00913.x

Schafer, J. L., \& Graham, J. W. (2002). Missing data: Our view of the state of the art. Psychological Methods, 7(2), 147-177. doi:10.1037//1082-989X.7.2.147

Schat, A. C. H., Kelloway, E. K., \& Desmarais, S. (2005). The Physical Health Questionnaire (PHQ): Construct validation of a self-report scale of somatic symptoms. Journal of Occupational Health Psychology, 10(4), 363-381. doi:10.1037/1076-8998.10.4.363

Schneider, B. (1987). The people make the place. Personnel Psychology, 40(3), 437-453. doi:10.1111/j.1744-6570.1987.tb00609.x

Schneider, B. (2001). Fits about fit. Applied Psychology, 50(1), 141-152. doi:10.1111/1464-0597.00051

Schneider, B., Kristof-Brown, A. L., Goldstein, H. W., \& Smith, D. B. (1997). What is this thing called fit? In N. Anderson \& P. Herriot (Eds.), International handbook of selection and assessment (pp. 393-412). Chichester, UK: John Wiley \& Sons.

Scott, W. E. (1966). Activation theory and task design. Organizational Behavior and Human Performance, 1(1), 3-30. doi:10.1016/0030-5073(66)90003-1

Sekaran, U. (1989). Paths to the job satisfaction of bank employees. Journal of Organizational Behavior. doi:10.1002/job.4030100405

Shakespeare-Finch, J., Wehr, T., Kaiplinger, I., \& Daley, E. (2014, May). Caring for emergency services personnel: Does what we do work? Paper session presented at the Australia and New Zealand Disaster and Emergency Management Conference, Surfers Paradise, QLD, Australia.

Shanock, L. R., Baran, B. E., Gentry, W. A., Pattison, S. C., \& Heggestad, E. D. (2010). Polynomial regression with response surface analysis: A powerful approach for examining moderation and overcoming limitations of difference scores. Journal of Business and Psychology, 25(4), 543-554. doi:10.1007/s10869-010-9183-4

Shuler, S. (2001). Talking community at 911 : The centrality of communication in coping with emotional labor. In G. J. Shepherd \& E. W. Rothenbuhler (Eds.), Communication and community (pp. 53-77). Mahwah, NJ: Lawrence Erlbaum. 
Silverthorne, C. (2004). The impact of organizational culture and person-organization fit on organizational commitment and job satisfaction in Taiwan. Leadership \& Organization Development Journal, 25(7), 592-599. doi:10.1108/01437730410561477

Spector, P. E. (1985). Higher-order need strength as a moderator of the job scopeemployee outcome relationship: A meta-analysis. Journal of Occupational Psychology, 58(2), 119-127. doi:10.1111/j.2044-8325.1985.tb00187.x

Spector, P. E. (1986). Perceived control by employees: A meta-analysis of studies concerning autonomy and participation at work. Human Relations, 39(11), 10051016. doi:10.1177/001872678603901104

Spector, P. E. (1994). Using self-report questionnaires in OB research: A comment on the use of a controversial method. Journal of Organizational Behavior, 15(5), 385392. doi:10.1002/job.4030150503

Spector, P. E., \& Jex, S. M. (1998). Development of four self-report measures of job stressors and strain: Interpersonal Conflict at Work Scale, Organizational Constraints Scale, Quantitative Workload Inventory, and Physical Symptoms Inventory. Journal of Occupational Health Psychology, 3(4), 356-67. doi:10.1037/1076-8998.3.4.356

Stamm, B. H. (2002). Measuring compassion satisfaction as well as fatigue: Developmental history of the Compassion Satisfaction and Fatigue test. In C. R. Figley (Ed.), Treating compassion fatigue (pp. 107-119). New York: Routledge.

Stamm, B. H. (2010). The concise ProQOL manual. ProQOL.org. Pocatello, ID. Retrieved from http://proqol.org/uploads/ProQOL_Concise_2ndEd_12-2010.pdf

Tafvelin, S., von Thiele Schwarz, U., \& Hasson, H. (2017). In agreement? Leader-team perceptual distance in organizational learning affects work performance. Journal of Business Research, 75, 1-7. doi:10.1016/j.jbusres.2017.01.016

Terborg, J. R. (1981). Interactional psychology and research on human behavior in organizations. The Academy of Management Review, 6(4), 569-576. doi: $10.2307 / 257635$

U.S. Department of Health and Human Services [DHHS], National Institute for Occupational Safety and Health [NIOSH]. (1999). Stress at work. (NIOSH Publication No. 99-101). Retrieved from http://www.cdc.gov/niosh/jobstres.html 
U.S. Department of Labor [DOL], Bureau of Labor Statistics [BLS]. (2015).

Occupational outlook handbook. Retrieved from

https://www.bls.gov/ooh/home.htm

Violanti, J. M., Andrew, M., Burchfiel, C. M., Hartley, T. A., Charles, L. E., \& Miller, D. B. (2007). Post-traumatic stress symptoms and cortisol patterns among police officers. Policing: An International Journal of Police Strategies \& Management, 30(2), 189-202. doi:10.1108/13639510710753207

Vroom, V. H. (1964). Work and motivation. Oxford, England: Wiley.

Wall, T. D., \& Payne, R. (1973). Are deficiency scores deficient? Journal of Applied Psychology, 58(3), 322-326. doi:10.1037/h0036227

Warr, P. (1987). Work unemployment and mental health. Oxford, England: Clarendon Press.

Warr, P. (1990). The measurement of well-being and other aspects of mental health. Journal of Occupational Psychology, 63(3), 193-210. doi:10.1111/j.20448325.1990.tb00521.x

Warr, P. (2005). Work, well-being, and mental health. In J. Barling, E. K. Kelloway, \& M. R. Frone (Eds.), Handbook of work stress (pp. 547-574). Thousand Oaks, CA: Sage. doi:10.4135/9781412975995.n23

White, R. W. (1959). Motivation reconsidered: The concept of competence. Psychological Review, 66(5), 297-333. doi:10.1037/h0040934

Xie, J. L., \& Johns, G. (1995). Job scope and stress: Can job scope be too high? The Academy of Management Journal, 38(5), 1288-1309. Retrieved from http://www.jstor.org/stable/256858

Zaniboni, S., Truxillo, D. M., \& Fraccaroli, F. (2013). Differential effects of task variety and skill variety on burnout and turnover intentions for older and younger workers. European Journal of Work and Organizational Psychology, 22(3), 306317. doi:10.1080/1359432X.2013.782288 
APPENDICES 
Appendix A - Tables

Table A1

Correlations between PE Fit Differences Scores' Transformations and Strains

\begin{tabular}{|c|c|c|c|c|c|c|}
\hline Strain Outcome & \multicolumn{6}{|c|}{ Job Complexity } \\
\hline & $\mathrm{E}$ & $\mathrm{P}$ & Fit & Deficiency & Excess & Poor Fit \\
\hline Job Dissatisfaction & $-.31 * *$ & $-.30 * *$ & -.03 & $-.19 * *$ & $.19 * *$ & $.47 * *$ \\
\hline Workload Dissatisfaction & -.04 & $-.17 * *$ & $.15 * *$ & -.03 & $.32 * *$ & $.36^{* *}$ \\
\hline Boredom & $-.51 * *$ & $-.34 * *$ & $-.26 * *$ & $-.38 * *$ & .02 & $.51 * *$ \\
\hline Depression & -.09 & $-.12 *$ & .03 & -.09 & $.17 * *$ & $.22 * *$ \\
\hline Anxiety & .00 & -.05 & .06 & -.08 & $.21 * *$ & $.21 * *$ \\
\hline Somatic Complaints & -.11 & -.02 & -.11 & $-.19 * *$ & .04 & $.16^{* *}$ \\
\hline Strain Outcome & \multicolumn{6}{|c|}{ Role Ambiguity } \\
\hline & E & $\mathrm{P}$ & Fit & Deficiency & Excess & Poor Fit \\
\hline Job Dissatisfaction & $.17 * *$ & .04 & .07 & -.01 & $.16^{* *}$ & $.19 * *$ \\
\hline Workload Dissatisfaction & $.16^{* *}$ & .09 & .03 & -.02 & .10 & $.13 *$ \\
\hline Boredom & .10 & .03 & .03 & -.04 & $.12 *$ & $.17^{* *}$ \\
\hline Depression & $.19 * *$ & .07 & .06 & .01 & .10 & $.12 *$ \\
\hline Anxiety & $.17 * *$ & .02 & .09 & .07 & .08 & .01 \\
\hline Somatic Complaints & .11 & .05 & .03 & .03 & .02 & .02 \\
\hline Strain Outcome & \multicolumn{6}{|c|}{ Responsibility for Persons } \\
\hline & $\mathrm{E}$ & $\mathrm{P}$ & Fit & Deficiency & Excess & Poor Fit \\
\hline Job Dissatisfaction & $-.26 * *$ & $-.15 * *$ & $-.14 *$ & $-.18 * *$ & .02 & $.23 * *$ \\
\hline Workload Dissatisfaction & -.05 & $-.15^{* *}$ & .06 & -.00 & $.17 * *$ & .07 \\
\hline Boredom & $-.30 * *$ & -.07 & $-.24 * *$ & $-.29 * *$ & -.03 & $.32 * *$ \\
\hline Depression & $-.13 *$ & $-.16^{* *}$ & .01 & -.02 & .08 & .05 \\
\hline Anxiety & -.04 & -.11 & .05 & .01 & $.12 *$ & .05 \\
\hline Somatic Complaints & $-.13 *$ & -.03 & -.09 & -.09 & -.05 & .07 \\
\hline Strain Outcome & \multicolumn{6}{|c|}{ Workload } \\
\hline & $\mathrm{E}$ & $\mathrm{P}$ & Fit & Deficiency & Excess & Poor Fit \\
\hline Job Dissatisfaction & .01 & $-.26 * *$ & $.19^{* *}$ & .07 & $.21 * *$ & $.22 * *$ \\
\hline Workload Dissatisfaction & $.33 * *$ & $-.34 * *$ & $.52 * *$ & $.26^{* *}$ & $.54 * *$ & $.36^{* *}$ \\
\hline Boredom & $-.19 * *$ & $-.28 * *$ & .05 & .02 & .06 & $.12 *$ \\
\hline Depression & .05 & $-.29 * *$ & $.27 * *$ & $.16^{* *}$ & $.26^{* *}$ & $.24 * *$ \\
\hline Anxiety & .05 & -.11 & $.13^{*}$ & .05 & $.15^{* *}$ & .09 \\
\hline Somatic Complaints & .00 & -.06 & .05 & .06 & .06 & .06 \\
\hline
\end{tabular}

Notes: $N=299$ - 310; E = reported environmental level; $\mathrm{P}=$ reported preferred level; Fit = E - P;

Deficiency $=\mathrm{E}-\mathrm{P}$ (for values less than or equal to 0 ) and Deficiency $=0$ (for values more than 0 );

Excess $=\mathrm{E}-\mathrm{P}$ (for values more than or equal to 0 ), and Excess $=0$ (for values less than 0 ); and Poor Fit

$=|\mathrm{E}-\mathrm{P}| ; * p<.05 ; * * p .01$ 
Table A2

Occupational Outlook for Emergency Services Personnel

\begin{tabular}{lcccc}
\hline & Police and Sheriff's PO & Firefighters & Dispatchers & EMT/Paramedics \\
\hline Median Annual Income (2015) & $\$ 60,270$ & $\$ 46,870$ & $\$ 38,010$ & $\$ 31,980$ \\
Workforce size projection & 806,400 & 327,300 & 102,000 & 241,200 \\
Projected growth rate (avg is 7\%) & $4 \%$ & $5 \%$ & $-3 \%$ & $24 \%$ \\
\% of population & $0.5 \%$ & $0.2 \%$ & $0.06 \%$ & $0.2 \%$ \\
\hline
\end{tabular}

Note: Population estimated at 162,465,000; PO = police officers, EMT = emergency medical technicians; Bureau of Labor Statistics, U.S. Department of Labor, Bureau of Labor Statistics (Publish Date: December 17, 2015), Occupational Outlook Handbook. Retrieved (on Oct 18, 2016) from www.bls.gov/ooh/ 
Table A3

Intercorrelations, Means, and Standard Deviations for Study Variables

\begin{tabular}{|c|c|c|c|c|c|c|c|c|c|c|c|}
\hline & Variables & $M$ & $M_{s c}$ & $S D$ & 1 & 2 & 3 & 4 & 5 & 6 & 7 \\
\hline 1 & Gender $^{1}$ & 0.251 & & 0.434 & - & & & & & & \\
\hline 2 & Age & 44.792 & & 9.272 & -.069 & - & & & & & \\
\hline 3 & Organizational tenure & 14.755 & & 8.569 & -.105 & $.615^{* *}$ & - & & & & \\
\hline 4 & Occupational tenure & 18.944 & & 9.193 & $-.191^{* *}$ & $.771^{* *}$ & $.688^{* *}$ & - & & & \\
\hline 5 & Skill variety environment & 5.656 & 1.656 & 1.059 & -.045 & -.074 & -.078 & .041 & .592 & & \\
\hline 6 & Skill variety person & 5.905 & 1.905 & 0.863 & -.021 & .070 & .006 & .063 & $.473^{* *}$ & .608 & \\
\hline 7 & Task identity environment & 4.617 & 0.617 & 1.176 & $-.189^{* *}$ & .095 & .032 & .076 & $.204^{* *}$ & .084 & .533 \\
\hline 8 & Task identity person & 5.403 & 1.403 & 1.135 & -.116 & -.001 & -.002 & -.076 & .031 & .096 & $.395^{* *}$ \\
\hline 9 & Task significance environment & 6.314 & 2.314 & 0.920 & .087 & -.023 & -.055 & .019 & $.465^{* *}$ & $.313^{* *}$ & .078 \\
\hline 10 & Task significance person & 5.964 & 1.964 & 0.976 & -.028 & .060 & .031 & .097 & $.382^{* *}$ & $.591^{* *}$ & .039 \\
\hline 11 & Autonomy environment & 5.087 & 1.087 & 1.121 & $-.261^{* *}$ & .023 & .058 & $.218^{* *}$ & $.242^{* *}$ & $.128^{*}$ & $.271^{* *}$ \\
\hline 12 & Autonomy person & 5.853 & 1.853 & 0.920 & $-.202^{* *}$ & -.019 & .056 & .095 & .093 & $.352^{* *}$ & $.154^{* *}$ \\
\hline 13 & Feedback environment & 4.776 & 0.776 & 1.171 & $-.137^{*}$ & -.004 & .017 & .021 & $.349^{* *}$ & $.213^{* *}$ & $.193^{* *}$ \\
\hline 14 & Feedback person & 5.470 & 1.470 & 0.927 & -.075 & .017 & $.152^{*}$ & -.023 & .047 & $.292^{* *}$ & $.164^{* *}$ \\
\hline 15 & Physical symptoms ${ }^{2}$ & 24.815 & & 8.736 & $.123^{*}$ & .022 & .038 & -.026 & $-.138^{*}$ & -.094 & $-.226^{* *}$ \\
\hline 16 & Burnout & 2.344 & & 0.676 & .029 & -.093 & .073 & -.042 & $-.281^{* *}$ & $-.226^{* *}$ & $-.242^{* *}$ \\
\hline 17 & Secondary traumatic stress & 2.332 & & 0.748 & .020 & -.077 & .070 & .002 & -.047 & $-.116^{*}$ & $-.202^{* *}$ \\
\hline 18 & Compassion satisfaction & 4.024 & & 0.675 & .019 & .018 & -.060 & .023 & $.447^{* *}$ & $.367^{* *}$ & $.198^{* *}$ \\
\hline
\end{tabular}

Notes: $M_{s c}=$ Mean for scale centered predictor variable, outcome variables were not scale centered; inter item reliabilities on diagonal; ${ }^{1}$ male $=0$, female $=1 ;{ }^{2}$ As a frequency count of symptoms, inter item reliability for Physical Symptoms Index was not appropriate for this scale (Spector \& Jex, 1998); $N=277-358 ; *=p<.05, * *=p<.01$ 
Table A3 (continued)

Intercorrelations, Means, and Standard Deviations for Study Variables

\begin{tabular}{|c|c|c|c|c|c|c|c|c|c|c|c|c|}
\hline & Variables & 8 & 9 & 10 & 11 & 12 & 13 & 14 & 15 & 16 & 17 & 18 \\
\hline 1 & Gender $^{1}$ & & & & & & & & & & & \\
\hline 2 & Age & & & & & & & & & & & \\
\hline 3 & Organizational tenure & & & & & & & & & & & \\
\hline 4 & Occupational tenure & & & & & & & & & & & \\
\hline 5 & Skill variety environment & & & & & & & & & & & \\
\hline 6 & Skill variety person & & & & & & & & & & & \\
\hline 7 & Task identity environment & & & & & & & & & & & \\
\hline 8 & Task identity person & .825 & & & & & & & & & & \\
\hline 9 & Task significance environment & -.005 & .615 & & & & & & & & & \\
\hline 10 & Task significance person & .079 & $.378^{* *}$ & .643 & & & & & & & & \\
\hline 11 & Autonomy environment & .070 & $.157^{* *}$ & $.209^{* *}$ & .599 & & & & & & & \\
\hline 12 & Autonomy person & $.294^{* *}$ & .094 & $.272^{* *}$ & $.390^{* *}$ & .735 & & & & & & \\
\hline 13 & Feedback environment & .068 & $.284^{* *}$ & $.268^{* *}$ & $.263^{* *}$ & .088 & .699 & & & & & \\
\hline 14 & Feedback person & $.327^{* *}$ & .075 & $.341^{* *}$ & .090 & $.345^{* *}$ & $.286^{* *}$ & .738 & & & & \\
\hline 15 & Physical symptoms ${ }^{2}$ & -.027 & $-.138^{*}$ & -.108 & $-.156^{* *}$ & -.073 & $-.253^{* *}$ & $-.136^{*}$ & - & & & \\
\hline 16 & Burnout & .017 & $-.224^{* *}$ & $-.276^{* *}$ & $-.209^{* *}$ & .013 & $-.404^{* *}$ & $-.193^{* *}$ & $.605^{* *}$ & .827 & & \\
\hline 17 & Secondary traumatic stress & .087 & -.067 & $-.148^{* *}$ & $-.148^{* *}$ & .009 & $-.266^{* *}$ & -.069 & $.514^{* *}$ & $.688^{* *}$ & .876 & \\
\hline 18 & Compassion satisfaction & .070 & $.364^{* *}$ & $.444^{* *}$ & $.241^{* *}$ & .083 & $.365^{* *}$ & $.302^{* *}$ & $-.346^{* *}$ & $-.694^{* *}$ & $-.275^{* *}$ & .923 \\
\hline
\end{tabular}

Notes: $M_{s c}=$ Mean for scale centered predictor variable, outcome variables were not scale centered; inter item reliabilities on diagonal; ${ }^{1}$ male $=0$, female $=1 ;{ }^{2}$ As a frequency count of symptoms, inter item reliability for Physical Symptoms Index was not appropriate for this scale (Spector \& Jex, 1998); $N=277-358 ; *=p<.05, * *=p<.01$ 
Table A4

Regression of Physical Symptoms on Skill Variety Fit

\begin{tabular}{lccccr}
\hline & \multicolumn{5}{c}{ Physical symptoms } \\
\cline { 2 - 6 } Variable & $b$ & $S E$ & $t$ & \multicolumn{2}{c}{ CI95 } \\
\hline Constant & $25.94^{* *}$ & 1.644 & 15.776 & 22.717 & 29.161 \\
SvE & -0.063 & 1.526 & -0.041 & -3.054 & 2.928 \\
SvP & -0.06 & 1.841 & -0.032 & -3.667 & 3.549 \\
SvE2 & 0.144 & 0.424 & 0.339 & -0.687 & 0.975 \\
SvEP & -0.692 & 0.71 & -0.976 & -2.085 & 0.699 \\
SvP2 & 0.198 & 0.706 & 0.281 & -1.186 & 1.582 \\
& & & & & \\
Surface tests & & & & & \\
\hline a1 & -0.123 & 1.755 & -0.070 & -3.563 & 3.317 \\
a2 & -0.350 & 0.538 & -0.651 & -1.404 & 0.704 \\
a3 & -0.003 & 2.891 & -0.001 & -5.669 & 5.663 \\
a4 & 1.034 & 1.439 & 0.719 & -1.786 & 3.854 \\
\hline
\end{tabular}

Notes: $N=358 ; \mathrm{SvE}=$ skill variety environment; $\mathrm{SvP}=$ skill variety person; $\mathrm{SvE} 2$ = skill variety environment squared; SvEP = cross-product of skill variety environment $\mathrm{x}$ skill variety person; SvP2 = skill variety person squared; $a 1=(b 1+b 2)$, where $b 1$ is beta coefficient for $\mathrm{SvE}$ and $b 2$ is beta coefficient for SvP; $a 2=(b 3+b 4+b 5)$ where $b 3$ is beta coefficient for SvE2, $b 4$ is beta coefficient for $\mathrm{SvEP}$, and $b 5$ is coefficient for SvP2; $a 3=(b 1-b 2) ; a 4=(b 3-b 4+b 5)$;

$*=p<.05, * *=p<.001$. 
Table A5

Regression of Burnout on Skill Variety Fit

\begin{tabular}{lccccc}
\hline & \multicolumn{5}{c}{ Burnout } \\
\cline { 2 - 6 } Variable & $b$ & $S E$ & $t$ & \multicolumn{2}{c}{ CI95 } \\
\hline Constant & 2.777 & 0.108 & 25.759 & 2.565 & 2.989 \\
SvE & -0.113 & 0.098 & -1.148 & -0.305 & 0.079 \\
SvP & -0.053 & 0.136 & -0.388 & -0.320 & 0.214 \\
SvE2 & -0.036 & 0.027 & -1.361 & -0.089 & 0.017 \\
SvEP & 0.026 & 0.048 & 0.532 & -0.068 & 0.120 \\
SvP2 & -0.022 & 0.051 & -0.426 & -0.122 & 0.078 \\
& & & & & \\
Surface tests & & & & & \\
\hline$a 1$ & -0.166 & 0.127 & -1.305 & -0.415 & 0.083 \\
a2 & -0.032 & 0.040 & -0.807 & -0.110 & 0.046 \\
a3 & -0.060 & 0.201 & -0.301 & -0.454 & 0.334 \\
$a 4$ & -0.084 & 0.097 & -0.861 & -0.274 & 0.106 \\
\hline
\end{tabular}

Notes: $N=358 ; \mathrm{SvE}=$ skill variety environment; $\mathrm{SvP}=$ skill variety person; $\mathrm{SvE} 2$ = skill variety environment squared; SvEP = cross-product of skill variety environment $\mathrm{x}$ skill variety person; SvP2 = skill variety person squared; $a 1=(b 1+b 2)$, where $b 1$ is beta coefficient for $\mathrm{SvE}$ and $b 2$ is beta coefficient for SvP; $a 2=(b 3+b 4+b 5)$ where $b 3$ is beta coefficient for SvE2, $b 4$ is beta coefficient for $\mathrm{SvEP}$, and $b 5$ is coefficient for $\mathrm{SvP} 2 ; a 3=(b 1-b 2) ; a 4=(b 3-b 4+b 5)$;

$*=p<.05, * *=p<.001$. 
Table A6

Regression of Secondary Traumatic Stress on Skill Variety Fit

\begin{tabular}{lccccc}
\hline & \multicolumn{5}{c}{ Secondary traumatic stress } \\
\cline { 2 - 6 } Variable & $b$ & \multicolumn{1}{c}{$S E$} & \multicolumn{3}{c}{ CI95 } \\
\hline Constant & 2.617 & 0.150 & 17.431 & 2.323 & 2.911 \\
SvE & -0.028 & 0.110 & -0.255 & -0.244 & 0.188 \\
SvP & -0.170 & 0.169 & -1.005 & -0.501 & 0.161 \\
SvE2 & -0.030 & 0.029 & -1.032 & -0.087 & 0.027 \\
SvEP & 0.053 & 0.049 & 1.092 & -0.043 & 0.149 \\
SvP2 & 0.002 & 0.056 & 0.027 & -0.108 & 0.112 \\
& & & & & \\
Surface tests & & & & & \\
\hline al & -0.198 & 0.184 & -1.075 & -0.559 & 0.163 \\
a2 & 0.025 & 0.055 & 0.457 & -0.083 & 0.133 \\
a3 & 0.142 & 0.218 & 0.649 & -0.285 & 0.569 \\
a4 & -0.081 & 0.093 & -0.874 & -0.263 & 0.101 \\
\hline
\end{tabular}

Notes: $N=358 ; \mathrm{SvE}=$ skill variety environment; $\mathrm{SvP}=$ skill variety person; $\mathrm{SvE} 2$ = skill variety environment squared; SvEP = cross-product of skill variety environment $\mathrm{x}$ skill variety person; SvP2 = skill variety person squared; $a 1=(b 1+b 2)$, where $b 1$ is beta coefficient for SvE and $b 2$ is beta coefficient for SvP; $a 2=(b 3+b 4+b 5)$ where $b 3$ is beta coefficient for SvE2, $b 4$ is beta coefficient for $\mathrm{SvEP}$, and $b 5$ is coefficient for SvP2; $a 3=(b 1-b 2) ; a 4=(b 3-b 4+b 5)$;

$*=p<.05, * *=p<.001$. 
Table A7

Regression of Compassion Satisfaction on Skill Variety Fit

\begin{tabular}{lccrrr}
\hline & \multicolumn{5}{c}{ Compassion satisfaction } \\
\cline { 2 - 6 } Variable & $b$ & $S E$ & \multicolumn{1}{c}{$t$} & \multicolumn{2}{c}{ CI95 } \\
\hline Constant & $3.305^{* *}$ & 0.150 & 21.998 & 3.011 & 3.599 \\
SvE & $0.284^{*}$ & 0.116 & 2.460 & 0.057 & 0.511 \\
SvP & 0.081 & 0.165 & 0.492 & -0.242 & 0.404 \\
SvE2 & 0.019 & 0.032 & 0.581 & -0.044 & 0.082 \\
SvEP & -0.054 & 0.061 & -0.879 & -0.174 & 0.066 \\
SvP2 & 0.048 & 0.059 & 0.811 & -0.068 & 0.164 \\
& & & & & \\
Surface tests & & & & & \\
\hline a1 & $0.366^{*}$ & 0.175 & 2.086 & 0.023 & 0.709 \\
a2 & 0.012 & 0.049 & 0.250 & -0.084 & 0.108 \\
a3 & 0.203 & 0.225 & 0.904 & -0.238 & 0.644 \\
a4 & 0.120 & 0.121 & 0.991 & -0.117 & 0.357 \\
\hline
\end{tabular}

Notes: $N=358 ; \mathrm{SvE}=$ skill variety environment; $\mathrm{SvP}=$ skill variety person; $\mathrm{SvE} 2$ = skill variety environment squared; SvEP = cross-product of skill variety environment $\mathrm{x}$ skill variety person; SvP2 = skill variety person squared; $a 1=(b 1+b 2)$, where $b 1$ is beta coefficient for SvE and $b 2$ is beta coefficient for SvP; $a 2=(b 3+b 4+b 5)$ where $b 3$ is beta coefficient for SvE2, $b 4$ is beta coefficient for $\mathrm{SvEP}$, and $b 5$ is coefficient for SvP2; $a 3=(b 1-b 2) ; a 4=(b 3-b 4+b 5)$;

$*=p<.05, * *=p<.001$. 
Table A8

Regression of Physical Symptoms on Task Identity Fit

\begin{tabular}{lccccr}
\hline & \multicolumn{5}{c}{ Physical symptoms } \\
\cline { 2 - 6 } Variable & $b$ & $S E$ & $t$ & \multicolumn{2}{c}{ C195 } \\
\hline Constant & $25.087^{* *}$ & 0.88 & 28.523 & 23.362 & 26.812 \\
TiE & -0.724 & 0.826 & -0.877 & -2.343 & 0.895 \\
TiP & 0.051 & 0.898 & 0.057 & -1.709 & 1.811 \\
TiE2 & -0.289 & 0.27 & -1.07 & -0.818 & 0.240 \\
TiEP & -0.569 & 0.447 & -1.275 & -1.445 & 0.307 \\
TiP2 & 0.395 & 0.348 & 1.136 & -0.287 & 1.077 \\
& & & & & \\
Surface tests & & & & & \\
\hline al & -0.673 & 1.309 & -0.514 & -3.239 & 1.893 \\
a2 & -0.463 & 0.510 & -0.908 & -1.463 & 0.537 \\
a3 & -0.775 & 1.123 & -0.690 & -2.976 & 1.426 \\
$a 4$ & 0.675 & 0.709 & 0.952 & -0.715 & 2.065 \\
\hline
\end{tabular}

Notes: $N=358$; TiE = task identity environment; TiP = task identity person; TiE2 = task identity environment squared; TiEP = cross-product of task identity environment $\mathrm{x}$ task identity person; TiP2 = task identity person squared; $a 1=(b 1+b 2)$, where $b 1$ is beta coefficient for TiE and $b 2$ is beta coefficient for TiP; $a 2=(b 3+b 4+b 5)$ where $b 3$ is beta coefficient for TiE2, $b 4$ is beta coefficient for TiEP, and $b 5$ is coefficient for TiP2; $a 3=(b 1-b 2) ; a 4=(b 3-b 4+b 5)$;

$*=p<.05, * *=p<.001$. 
Table A9

Regression of Burnout on Task Identity Fit

\begin{tabular}{lccccc}
\hline & \multicolumn{5}{c}{ Burnout } \\
\cline { 2 - 6 } Variable & $b$ & $S E$ & $t$ & \multicolumn{2}{c}{ CI95 } \\
\hline Constant & $2.318^{* *}$ & 0.063 & 36.751 & 2.195 & 2.441 \\
TiE & -0.02 & 0.065 & -0.312 & -0.147 & 0.107 \\
TiP & $0.117^{*}$ & 0.056 & 2.079 & 0.007 & 0.227 \\
TiE2 & 0.006 & 0.024 & 0.262 & -0.041 & 0.053 \\
TiEP & -0.085 & 0.037 & -2.297 & -0.158 & -0.012 \\
TiP2 & -0.004 & 0.023 & -0.162 & -0.049 & 0.041 \\
& & & & & \\
Surface tests & & & & & \\
\hline$a 1$ & 0.096 & 0.085 & 1.126 & -0.071 & 0.263 \\
a2 & $-0.083^{*}$ & 0.036 & -2.298 & -0.154 & -0.012 \\
a3 & -0.137 & 0.087 & -1.575 & -0.308 & 0.034 \\
a4 & 0.087 & 0.061 & 1.442 & -0.033 & 0.207 \\
\hline
\end{tabular}

Notes: $N=358$; TiE = task identity environment; TiP = task identity person; TiE2 = task identity environment squared; TiEP = cross-product of task identity environment $\mathrm{x}$ task identity person; TiP2 = task identity person squared; $a 1=(b 1+b 2)$, where $b 1$ is beta coefficient for TiE and $b 2$ is beta coefficient for TiP; $a 2=(b 3+b 4+b 5)$ where $b 3$ is beta coefficient for TiE2, $b 4$ is beta coefficient for TiEP, and $b 5$ is coefficient for TiP2; $a 3=(b 1-b 2) ; a 4=(b 3-b 4+b 5)$;

$*=p<.05, * *=p<.001$. 
Table A10

Regression of Secondary Traumatic Stress on Task Identity Fit

\begin{tabular}{lcrrrr}
\hline & \multicolumn{5}{c}{ Secondary traumatic stress } \\
\cline { 2 - 6 } Variable & \multicolumn{1}{c}{$S E$} & \multicolumn{1}{c}{$t$} & \multicolumn{2}{c}{ CI95 } \\
\hline Constant & $2.246^{* *}$ & 0.066 & 34.233 & 2.117 & 2.375 \\
TiE & -0.068 & 0.066 & -1.026 & -0.197 & 0.061 \\
TiP & $0.141^{*}$ & 0.045 & 3.129 & 0.053 & 0.229 \\
TiE2 & -0.001 & 0.028 & -0.035 & -0.056 & 0.054 \\
TiEP & -0.061 & 0.042 & -1.462 & -0.143 & 0.021 \\
TiP2 & 0.005 & 0.025 & 0.211 & -0.044 & 0.054
\end{tabular}

Surface tests

\begin{tabular}{lllrrr}
\hline$a 1$ & 0.074 & 0.067 & 1.092 & -0.057 & 0.205 \\
a2 & -0.056 & 0.034 & -1.644 & -0.123 & 0.011 \\
a3 & $-0.209 *$ & 0.091 & -2.301 & -0.387 & -0.031 \\
a4 & 0.065 & 0.073 & 0.887 & -0.078 & 0.208 \\
\hline
\end{tabular}

Notes: $N=358$; TiE = task identity environment; TiP = task identity person; TiE2 = task identity environment squared; TiEP = cross-product of task identity environment $\mathrm{x}$ task identity person; TiP2 = task identity person squared; $a 1=(b 1+b 2)$, where $b 1$ is beta coefficient for TiE and $b 2$ is beta coefficient for TiP; $a 2=(b 3+b 4+b 5)$ where $b 3$ is beta coefficient for TiE2, $b 4$ is beta coefficient for TiEP, and $b 5$ is coefficient for TiP2; $a 3=(b 1-b 2) ; a 4=(b 3-b 4+b 5)$;

$*=p<.05, * *=p<.001$. 
Table A11

Regression of Compassion Satisfaction on Task Identity Fit

\begin{tabular}{lcrrrr}
\hline & \multicolumn{5}{c}{ Compassion satisfaction } \\
\cline { 2 - 6 } Variable & $b$ & \multicolumn{1}{c}{$S E$} & \multicolumn{1}{c}{$t$} & \multicolumn{2}{c}{ CI95 } \\
\hline Constant & $3.967^{* *}$ & 0.07 & 56.766 & 3.830 & 4.104 \\
TiE & 0.031 & 0.072 & 0.425 & -0.110 & 0.172 \\
TiP & -0.067 & 0.067 & -0.991 & -0.198 & 0.064 \\
TiE2 & -0.014 & 0.026 & -0.532 & -0.065 & 0.037 \\
TiEP & 0.047 & 0.038 & 1.251 & -0.027 & 0.121 \\
TiP2 & 0.025 & 0.027 & 0.919 & -0.028 & 0.078 \\
& & & & & \\
Surface tests & & & & & \\
\hline$a 1$ & -0.036 & 0.104 & -0.349 & -0.240 & 0.168 \\
$a 2$ & 0.058 & 0.042 & 1.403 & -0.024 & 0.140 \\
$a 3$ & 0.097 & 0.093 & 1.045 & -0.085 & 0.279 \\
$a 4$ & -0.037 & 0.061 & -0.598 & -0.157 & 0.083 \\
\hline
\end{tabular}

Notes: $N=358$; TiE = task identity environment; TiP = task identity person; TiE2 = task identity environment squared; TiEP = cross-product of task identity environment $\mathrm{x}$ task identity person; TiP2 = task identity person squared; $a 1=(b 1+b 2)$, where $b 1$ is beta coefficient for TiE and $b 2$ is beta coefficient for TiP; $a 2=(b 3+b 4+b 5)$ where $b 3$ is beta coefficient for TiE2, $b 4$ is beta coefficient for TiEP, and $b 5$ is coefficient for TiP2; $a 3=(b 1-b 2) ; a 4=(b 3-b 4+b 5)$;

$*=p<.05, * *=p<.001$. 
Table A12

Regression of Physical Symptoms on Task Significance Fit

\begin{tabular}{lccrrr}
\hline & \multicolumn{5}{c}{ Physical symptoms } \\
\cline { 2 - 6 } Variable & $b$ & $S E$ & \multicolumn{1}{c}{$t$} & \multicolumn{2}{c}{ CI95 } \\
\hline Constant & $28.693^{* *}$ & 2.743 & 10.462 & 23.317 & 34.069 \\
TsE & -3.227 & 2.289 & -1.410 & -7.713 & 1.259 \\
TsP & -0.186 & 1.417 & -0.132 & -2.963 & 2.591 \\
TsEP & 0.711 & 0.617 & 1.153 & -0.498 & 1.920 \\
TsE2 & 0.246 & 0.688 & 0.358 & -1.102 & 1.594 \\
TsP2 & -0.376 & 0.470 & -0.801 & -1.297 & 0.545 \\
& & & & & \\
Surface tests & & & & & \\
\hline a1 & -3.414 & 2.289 & -1.491 & -7.900 & 1.072 \\
a2 & 0.581 & 0.567 & 1.026 & -0.530 & 1.692 \\
a3 & -3.041 & 3.043 & -0.999 & -9.005 & 2.923 \\
a4 & 0.088 & 1.303 & 0.068 & -2.466 & 2.642 \\
\hline
\end{tabular}

Notes: $N=358$; TsE $=$ task significance environment; $\mathrm{TsP}=$ task significance person; TsE2 $=$ task significance environment squared; TsEP $=$ cross-product of task significance environment $\mathrm{x}$ task significance person; $\mathrm{TsP} 2=$ task significance person squared; $a 1=(b 1+b 2)$, where $b 1$ is beta coefficient for TsE and $b 2$ is beta coefficient for TsP; $a 2=(b 3+b 4+b 5)$ where $b 3$ is beta coefficient for TsE2, $b 4$ is beta coefficient for TsEP, and $b 5$ is coefficient for TsP2; $a 3=(b 1-b 2) ; a 4=(b 3-b 4+$ b5);

$*=p<.05, * *=p<.001$. 
Table A13

Regression of Burnout on Task Significance Fit

\begin{tabular}{lccccc}
\hline & \multicolumn{5}{c}{ Burnout } \\
\cline { 2 - 6 } Variable & $b$ & $S E$ & $t$ & \multicolumn{2}{c}{ CI95 } \\
\hline Constant & $2.834^{* *}$ & 0.107 & 26.516 & 2.624 & 3.044 \\
TsE & -0.145 & 0.098 & -1.48 & -0.337 & 0.047 \\
TsP & -0.096 & 0.075 & -1.277 & -0.243 & 0.051 \\
TsEP & 0.033 & 0.031 & 1.068 & -0.028 & 0.094 \\
TsE2 & -0.046 & 0.038 & -1.205 & -0.120 & 0.028 \\
TsP2 & 0.012 & 0.03 & 0.392 & -0.047 & 0.071 \\
& & & & & \\
Surface tests & & & & & \\
\hline$a 1$ & $-0.241^{*}$ & 0.096 & -2.515 & -0.429 & -0.053 \\
a2 & -0.001 & 0.029 & -0.043 & -0.058 & 0.056 \\
$a 3$ & -0.050 & 0.146 & -0.339 & -0.336 & 0.236 \\
$a 4$ & 0.090 & 0.075 & 1.207 & -0.057 & 0.237 \\
\hline
\end{tabular}

Notes: $N=358$; $\mathrm{TsE}=$ task significance environment; $\mathrm{TsP}=$ task significance person; $\mathrm{TsE} 2=$ task significance environment squared; TsEP $=$ cross-product of task significance environment $\mathrm{x}$ task significance person; $\mathrm{TsP} 2=$ task significance person squared; $a 1=(b 1+b 2)$, where $b 1$ is beta coefficient for TsE and $b 2$ is beta coefficient for TsP; $a 2=(b 3+b 4+b 5)$ where $b 3$ is beta coefficient for TsE2, $b 4$ is beta coefficient for TsEP, and $b 5$ is coefficient for TsP2; $a 3=(b 1-b 2) ; a 4=(b 3-b 4+$ b5);

$*=p<.05, * *=p<.001$. 
Table A14

Regression of Secondary Traumatic Stress on Task Significance Fit

\begin{tabular}{lccccc}
\hline & \multicolumn{5}{c}{ Secondary traumatic stress } \\
\cline { 2 - 6 } Variable & $b$ & $S E$ & $t$ & \multicolumn{2}{c}{ CI95 } \\
\hline Constant & $2.513^{* *}$ & 0.179 & 14.056 & 2.162 & 2.864 \\
TsE & -0.100 & 0.170 & -0.587 & -0.433 & 0.233 \\
TsP & -0.073 & 0.111 & -0.658 & -0.291 & 0.145 \\
TsEP & 0.053 & 0.045 & 1.177 & -0.035 & 0.141 \\
TsE2 & -0.045 & 0.050 & -0.909 & -0.143 & 0.053 \\
TsP2 & 0.017 & 0.046 & 0.376 & -0.073 & 0.107 \\
& & & & & \\
Surface tests & & & & & \\
\hline a1 & -0.173 & 0.150 & -1.157 & -0.467 & 0.121 \\
a2 & 0.025 & 0.040 & 0.630 & -0.053 & 0.103 \\
a3 & -0.027 & 0.246 & -0.108 & -0.509 & 0.455 \\
a4 & 0.116 & 0.101 & 1.149 & -0.082 & 0.314 \\
\hline
\end{tabular}

Notes: $N=358$; TsE $=$ task significance environment; TsP $=$ task significance person; TsE2 $=$ task significance environment squared; TsEP $=$ cross-product of task significance environment $\mathrm{x}$ task significance person; $\mathrm{TsP} 2=$ task significance person squared; $a 1=(b 1+b 2)$, where $b 1$ is beta coefficient for TsE and $b 2$ is beta coefficient for TsP; $a 2=(b 3+b 4+b 5)$ where $b 3$ is beta coefficient for TsE2, $b 4$ is beta coefficient for TsEP, and $b 5$ is coefficient for TsP2; $a 3=(b 1-b 2) ; a 4=(b 3-b 4+$ b5);

$*=p<.05, * *=p<.001$. 
Table A15

Regression of Compassion Satisfaction on Task Significance Fit

\begin{tabular}{lcrrrr}
\hline \multirow{2}{*}{ Variable } & \multicolumn{5}{c}{ Compassion satisfaction } \\
\cline { 2 - 6 } Constant & \multicolumn{1}{c}{ b } & \multicolumn{1}{c}{$t$} & \multicolumn{2}{c}{ CI95 } \\
TsE & $3.117^{* *}$ & 0.143 & 21.783 & 2.837 & 3.397 \\
TsP & 0.235 & 0.150 & 1.566 & -0.059 & 0.529 \\
TsEP & $0.261^{*}$ & 0.105 & 2.478 & 0.055 & 0.467 \\
TsE2 & -0.020 & 0.046 & -0.436 & -0.110 & 0.070 \\
TsP2 & 0.012 & 0.051 & 0.228 & -0.088 & 0.112 \\
& -0.018 & 0.034 & -0.550 & -0.085 & 0.049 \\
Surface tests & & & & & \\
\hline$a 1$ & & & & & \\
a2 & & & & & \\
$a 3$ & -0.027 & 0.036 & -0.752 & -0.098 & 0.044 \\
$a 4$ & -0.027 & 0.221 & -0.120 & -0.460 & 0.406 \\
\hline
\end{tabular}

Notes: $N=358 ; \mathrm{TsE}=$ task significance environment; TsP $=$ task significance person; TsE2 $=$ task significance environment squared; TsEP $=$ cross-product of task significance environment $\mathrm{x}$ task significance person; $\mathrm{TsP} 2=$ task significance person squared; $a 1=(b 1+b 2)$, where $b 1$ is beta coefficient for TsE and $b 2$ is beta coefficient for TsP; $a 2=(b 3+b 4+b 5)$ where $b 3$ is beta coefficient for TsE2, $b 4$ is beta coefficient for TsEP, and $b 5$ is coefficient for TsP2; $a 3=(b 1-b 2) ; a 4=(b 3-b 4+$ b5);

$*=p<.05, * *=p<.001$. 
Table A16

Regression of Physical Symptoms on Autonomy Fit

\begin{tabular}{lccccc}
\hline & \multicolumn{5}{c}{ Physical symptoms } \\
\cline { 2 - 6 } Variable & $b$ & $S E$ & $t$ & \multicolumn{2}{c}{ CI95 } \\
\hline Constant & $26.562^{* *}$ & 1.721 & 15.432 & 23.189 & 29.935 \\
AuE & -0.349 & 1.212 & -0.288 & -2.725 & 2.027 \\
AuP & -1.591 & 1.899 & -0.838 & -5.313 & 2.131 \\
AuE2 & 0.026 & 0.375 & 0.071 & -0.709 & 0.761 \\
AuEP & -0.468 & 0.636 & -0.736 & -1.715 & 0.779 \\
AuP2 & 0.611 & 0.599 & 1.020 & -0.563 & 1.785 \\
& & & & & \\
Surface tests & & & & & \\
\hline al & -1.940 & 2.113 & -0.918 & -6.081 & 2.201 \\
a2 & 0.169 & 0.658 & 0.257 & -1.121 & 1.459 \\
a3 & 1.242 & 2.384 & 0.521 & -3.431 & 5.915 \\
a4 & 1.105 & 1.177 & 0.939 & -1.202 & 3.412 \\
\hline
\end{tabular}

Notes: predictor values are scale centered; $N=358 ; \mathrm{AuE}=$ autonomy environment; $\mathrm{AuP}=$ autonomy person; $\mathrm{AuE} 2$ = autonomy environment squared; $\mathrm{AuEP}$ = cross-product of autonomy environment $\mathrm{x}$ autonomy person; $\mathrm{AuP} 2=$ autonomy person squared; $a 1=(b 1+b 2)$, where $b 1$ is beta coefficient for $\mathrm{AuE}$ and $b 2$ is beta coefficient for AuP; $a 2=(b 3+b 4+b 5)$ where $b 3$ is beta coefficient for AuE2, $b 4$ is beta coefficient for AuEP, and $b 5$ is coefficient for AuP squared;

$*=p<.05, * *=p<.01$. 
Table A17

Regression of Burnout on Autonomy Fit

\begin{tabular}{lccrrr}
\hline & \multicolumn{5}{c}{ Burnout } \\
\cline { 2 - 6 } Variable & $b$ & $S E$ & \multicolumn{1}{c}{ CI95 } \\
\hline Constant & $2.359^{* *}$ & 0.097 & 24.267 & 2.169 & 2.549 \\
AuE & $-0.161^{*}$ & 0.078 & -2.064 & -0.314 & -0.008 \\
AuP & 0.033 & 0.091 & 0.364 & -0.145 & 0.211 \\
AuE2 & 0.006 & 0.017 & 0.319 & -0.027 & 0.039 \\
AuEP & 0.000 & 0.042 & -0.011 & -0.082 & 0.082 \\
AuP2 & 0.020 & 0.032 & 0.621 & -0.043 & 0.083 \\
& & & & & \\
Surface tests & & & & & \\
\hline$a 1$ & -0.128 & 0.113 & -1.131 & -0.349 & 0.093 \\
$a 2$ & 0.025 & 0.040 & 0.624 & -0.053 & 0.103 \\
$a 3$ & -0.195 & 0.127 & -1.533 & -0.444 & 0.054 \\
$a 4$ & 0.026 & 0.069 & 0.373 & -0.109 & 0.161 \\
\hline
\end{tabular}

Notes: predictor values are scale centered; $N=358 ; \mathrm{AuE}=$ autonomy environment; $\mathrm{AuP}=$ autonomy person; AuE2 = autonomy environment squared; AuEP = cross-product of autonomy environment $\mathrm{x}$ autonomy person; $\mathrm{AuP} 2=$ autonomy person squared; $a 1=(b 1+b 2)$, where $b 1$ is beta coefficient for $\mathrm{AuE}$ and $b 2$ is beta coefficient for AuP; $a 2=(b 3+b 4+b 5)$ where $b 3$ is beta coefficient for AuE2, $b 4$ is beta coefficient for AuEP, and $b 5$ is coefficient for AuP squared;

$*=p<.05, * *=p<.01$. 
Table A18

Regression of Secondary Traumatic Stress on Autonomy Fit

\begin{tabular}{lccccc}
\hline & \multicolumn{5}{c}{ Secondary traumatic stress } \\
\cline { 2 - 6 } Variable & $b$ & $S E$ & $t$ & \multicolumn{2}{c}{ CI95 } \\
\hline Constant & $2.314^{* *}$ & 0.089 & 26.101 & 2.140 & 2.488 \\
AuE & -0.088 & 0.095 & -0.933 & -0.274 & 0.098 \\
AuP & 0.055 & 0.071 & 0.777 & -0.084 & 0.194 \\
AuE2 & 0.004 & 0.024 & 0.173 & -0.043 & 0.051 \\
AuEP & -0.020 & 0.049 & -0.414 & -0.116 & 0.076 \\
AuP2 & 0.011 & 0.030 & 0.371 & -0.048 & 0.070 \\
& & & & & \\
Surface tests & & & & & \\
\hline$a 1$ & -0.034 & 0.109 & -0.310 & -0.248 & 0.180 \\
$a 2$ & -0.005 & 0.041 & -0.120 & -0.085 & 0.075 \\
$a 3$ & -0.143 & 0.127 & -1.128 & -0.392 & 0.106 \\
$a 4$ & 0.036 & 0.080 & 0.448 & -0.121 & 0.193 \\
\hline
\end{tabular}

Notes: predictor values are scale centered; $N=358 ; \mathrm{AuE}=$ autonomy environment; $\mathrm{AuP}=$ autonomy person; AuE2 = autonomy environment squared; AuEP = cross-product of autonomy environment $\mathrm{x}$ autonomy person; $\mathrm{AuP} 2=$ autonomy person squared; $a 1=(b 1+b 2)$, where $b 1$ is beta coefficient for $\mathrm{AuE}$ and $b 2$ is beta coefficient for AuP; $a 2=(b 3+b 4+b 5)$ where $b 3$ is beta coefficient for AuE2, $b 4$ is beta coefficient for AuEP, and $b 5$ is coefficient for AuP squared;

$*=p<.05, * *=p<.01$. 
Table A19

Regression of Compassion Satisfaction on Autonomy Fit

\begin{tabular}{lccccc}
\hline \multirow{2}{*}{ Variable } & \multicolumn{5}{c}{ Compassion satisfaction } \\
\cline { 2 - 6 } Constant & $b$ & $S E$ & $t$ & \multicolumn{2}{c}{ CI95 } \\
AuE & $3.858^{* *}$ & 0.126 & 30.689 & 3.611 & 4.105 \\
AuP & $0.206^{*}$ & 0.102 & 2.028 & 0.006 & 0.406 \\
AuE2 & -0.001 & 0.139 & -0.007 & -0.273 & 0.271 \\
AuEP & -0.006 & 0.021 & -0.283 & -0.047 & 0.035 \\
AuP2 & -0.026 & 0.052 & -0.501 & -0.128 & 0.076 \\
& 0.005 & 0.047 & 0.102 & -0.087 & 0.097 \\
Surface tests & & & & & \\
\hline$a 1$ & & & & & \\
$a 2$ & 0.205 & 0.158 & 1.297 & -0.105 & 0.515 \\
$a 3$ & -0.027 & 0.050 & -0.544 & -0.125 & 0.071 \\
$a 4$ & 0.207 & 0.185 & 1.120 & -0.156 & 0.570 \\
\hline
\end{tabular}

Notes: predictor values are scale centered; $N=358 ; \mathrm{AuE}=$ autonomy environment; $\mathrm{AuP}=$ autonomy person; AuE2 = autonomy environment squared; AuEP = cross-product of autonomy environment $\mathrm{x}$ autonomy person; $\mathrm{AuP} 2=$ autonomy person squared; $a 1=(b 1+b 2)$, where $b 1$ is beta coefficient for $\mathrm{AuE}$ and $b 2$ is beta coefficient for AuP; $a 2=(b 3+b 4+b 5)$ where $b 3$ is beta coefficient for AuE2, $b 4$ is beta coefficient for AuEP, and $b 5$ is coefficient for AuP squared;

$*=p<.05, * *=p<.01$. 
Table A20

Regression of Physical Symptoms on Feedback Fit

\begin{tabular}{lccccc}
\hline & \multicolumn{5}{c}{ Physical symptoms } \\
\cline { 2 - 6 } Variable & $b$ & $S E$ & $t$ & \multicolumn{2}{c}{ CI95 } \\
\hline Constant & $27.110^{* *}$ & 1.291 & 20.995 & 24.580 & 29.640 \\
FjE & $-2.338^{* *}$ & 0.891 & -2.625 & -4.084 & -0.592 \\
FjP & -1.469 & 1.476 & -0.995 & -4.362 & 1.424 \\
FjE2 & 0.112 & 0.322 & 0.349 & -0.519 & 0.743 \\
FjEP & 0.340 & 0.643 & 0.528 & -0.920 & 1.600 \\
FjP2 & 0.269 & 0.573 & 0.469 & -0.854 & 1.392 \\
& & & & & \\
Surface tests & & & & & \\
\hline a1 & & & & -6.957 & -0.657 \\
a2 & $-3.807 *$ & 1.607 & -2.369 & -0.320 & 1.762 \\
a3 & 0.721 & 0.531 & 1.357 & -4.464 & 2.726 \\
a4 & -0.869 & 1.834 & -0.474 & -2.366 & 2.448 \\
\hline
\end{tabular}

Notes: predictor values are scale centered; $N=358 ; \mathrm{FjE}=$ feedback environment; $\mathrm{FjP}=$ feedback person; FjE2 = feedback environment squared; FjEP = cross-product of feedback environment $\mathrm{x}$ feedback person; $\mathrm{FjP} 2=$ feedback person squared; $a 1=(b 1+b 2)$, where $b 1$ is beta coefficient for FjE and $b 2$ is beta coefficient for FjP; $a 2=(b 3+b 4+b 5)$ where $b 3$ is beta coefficient for FjE2, $b 4$ is beta coefficient for FjEP, and $b 5$ is coefficient for FjP2; $a 3=(b 1-b 2) ; a 4=(b 3-b 4+b 5)$;

$*=p<.05, * *=p<.01$. 
Table A21

Regression of Burnout on Feedback Fit

\begin{tabular}{lccccc}
\hline & \multicolumn{5}{c}{ Burnout } \\
\cline { 2 - 6 } Variable & $b$ & $S E$ & $t$ & \multicolumn{2}{c}{ CI95 } \\
\hline Constant & $2.645^{* *}$ & 0.071 & 37.01 & 2.506 & 2.784 \\
FjE & $-0.278^{* *}$ & 0.056 & -4.993 & -0.388 & -0.168 \\
FjP & -0.044 & 0.078 & -0.569 & -0.197 & 0.109 \\
FjE2 & -0.018 & 0.022 & -0.804 & -0.061 & 0.025 \\
FjEP & 0.049 & 0.034 & 1.429 & -0.018 & 0.116 \\
FjP2 & -0.018 & 0.03 & -0.587 & -0.077 & 0.041 \\
& & & & & \\
Surface tests & & & & & \\
\hline$a 1$ & $-0.323^{* *}$ & 0.090 & -3.604 & -0.499 & -0.147 \\
a2 & 0.014 & 0.038 & 0.368 & -0.060 & 0.088 \\
a3 & $-0.234^{*}$ & 0.101 & -2.314 & -0.432 & -0.036 \\
$a 4$ & -0.084 & 0.060 & -1.407 & -0.202 & 0.034 \\
\hline
\end{tabular}

Notes: predictor values are scale centered; $N=358 ; \mathrm{FjE}=$ feedback environment; $\mathrm{FjP}=$ feedback person; FjE2 = feedback environment squared; FjEP = cross-product of feedback environment $\mathrm{x}$ feedback person; $\mathrm{FjP} 2=$ feedback person squared; $a 1=(b 1+b 2)$, where $b 1$ is beta coefficient for FjE and $b 2$ is beta coefficient for FjP; $a 2=(b 3+b 4+b 5)$ where $b 3$ is beta coefficient for $\mathrm{FjE} 2, b 4$ is beta coefficient for FjEP, and $b 5$ is coefficient for FjP2; $a 3=(b 1-b 2) ; a 4=(b 3-b 4+b 5)$;

$*=p<.05, * *=p<.01$. 
Table A22

Regression of Secondary Traumatic Stress on Feedback Fit

\begin{tabular}{lccrrr}
\hline & \multicolumn{5}{c}{ Secondary traumatic stress } \\
\cline { 2 - 6 } Variable & $b$ & \multicolumn{1}{c}{$S E$} & \multicolumn{1}{c}{ CI95 } \\
\hline Constant & $2.466^{* *}$ & 0.077 & 32.091 & 2.315 & 2.617 \\
FjE & $-0.186^{* *}$ & 0.071 & -2.609 & -0.325 & -0.047 \\
FjP & 0.011 & 0.067 & 0.168 & -0.120 & 0.142 \\
FjE2 & -0.010 & 0.027 & -0.367 & -0.063 & 0.043 \\
FjEP & 0.016 & 0.046 & 0.341 & -0.074 & 0.106 \\
FjP2 & -0.004 & 0.033 & -0.118 & -0.069 & 0.061 \\
& & & & & \\
Surface tests & & & & & \\
\hline al & $-0.175 \dagger$ & 0.091 & -1.917 & -0.353 & 0.003 \\
a2 & 0.002 & 0.041 & 0.050 & -0.078 & 0.082 \\
a3 & $-0.198 \dagger$ & 0.104 & -1.898 & -0.402 & 0.006 \\
a4 & -0.030 & 0.080 & -0.368 & -0.187 & 0.127 \\
\hline
\end{tabular}

Notes: predictor values are scale centered; $N=358 ; \mathrm{FjE}=$ feedback environment; $\mathrm{FjP}=$ feedback person; FjE2 = feedback environment squared; FjEP = cross-product of feedback environment $\mathrm{x}$ feedback person; FjP2 = feedback person squared; $a 1=(b 1+b 2)$, where $b 1$ is beta coefficient for FjE and $b 2$ is beta coefficient for $\mathrm{FjP} ; a 2=(b 3+b 4+b 5)$ where $b 3$ is beta coefficient for $\mathrm{FjE} 2, b 4$ is beta coefficient for FjEP, and $b 5$ is coefficient for FjP2; $a 3=(b 1-b 2) ; a 4=(b 3-b 4+b 5)$;

$\dagger=p<.1 *=p<.05, * *=p<.01$. 
Table A23

Regression of Compassion Satisfaction on Feedback Fit

\begin{tabular}{lccrrr}
\hline & \multicolumn{5}{c}{ Compassion satisfaction } \\
\cline { 2 - 6 } Variable & $b$ & \multicolumn{1}{c}{$S E$} & \multicolumn{1}{c}{ CI95 } \\
\hline Constant & $3.628^{* *}$ & 0.086 & 42.278 & 3.459 & 3.797 \\
FjE & $0.273^{* *}$ & 0.071 & 3.840 & 0.134 & 0.412 \\
FjP & 0.118 & 0.096 & 1.230 & -0.070 & 0.306 \\
FjE2 & -0.005 & 0.026 & -0.179 & -0.056 & 0.046 \\
FjEP & -0.059 & 0.044 & -1.343 & -0.145 & 0.027 \\
FjP2 & 0.032 & 0.035 & 0.927 & -0.037 & 0.101 \\
& & & & & \\
Surface tests & & & & & \\
\hline a1 & & & & 0.171 & 0.611 \\
a2 & $0.391 * *$ & 0.112 & 3.502 & -0.112 & 0.048 \\
a3 & -0.032 & 0.041 & -0.774 & -0.092 & 0.402 \\
a4 & 0.155 & 0.126 & 1.228 & -0.068 & 0.242 \\
\hline
\end{tabular}

Notes: predictor values are scale centered; $N=358 ; \mathrm{FjE}=$ feedback environment; $\mathrm{FjP}=$ feedback person; FjE2 = feedback environment squared; FjEP = cross-product of feedback environment $\mathrm{x}$ feedback person; FjP2 = feedback person squared; $a 1=(b 1+b 2)$, where $b 1$ is beta coefficient for FjE and $b 2$ is beta coefficient for $\mathrm{FjP} ; a 2=(b 3+b 4+b 5)$ where $b 3$ is beta coefficient for FjE2, $b 4$ is beta coefficient for FjEP, and $b 5$ is coefficient for FjP2; $a 3=(b 1-b 2) ; a 4=(b 3-b 4+b 5)$;

$*=p<.05, * *=p<.01$. 
Table A24

Agreement and Discrepancy Percentages for Job Characteristics Dimensions

\begin{tabular}{lccc}
\hline & $\begin{array}{c}\text { Agreement } \\
\text { within } 1 S D\end{array}$ & & $\begin{array}{c}\text { Discrepancy } \\
\text { greater than } 1 S D\end{array}$ \\
\cline { 2 - 2 } Skill variety & $70.1 \%$ & & $29.9 \%$ \\
Task identity & $66.8 \%$ & $33.2 \%$ \\
Task significance & $67.9 \%$ & $32.1 \%$ \\
Autonomy & $70.1 \%$ & $29.9 \%$ \\
Feedback & $65.1 \%$ & $34.9 \%$ \\
\hline
\end{tabular}

Notes: Calculated by absolute difference between $z$-scores for person and environment components of each job characteristics dimension. 
Appendix B - Scales

Job Diagnostics Survey (JDS) - Hackman \& Oldham, 1980
Modified to assess person and environment components

Instructions: This questionnaire was developed as part of a Yale University study of jobs and how people react to them. The questionnaire helps to determine how jobs can be better designed, by obtaining information about how people react to different kinds of jobs.

On the following pages you will find several different kinds of questions about your job. Specific instructions are given at the start of each section. Please read them carefully. There are no trick questions. Your individual answers will be kept completely confidential. Please answer each item as honestly and frankly as possible. Thank you for your participation.

$\underline{\text { Person component }}$

JDS - Section 1P

Instructions: This part of the questionnaire asks for details about your preferences along these job characteristics.

In other words, if you worked in a similar position, perfectly suited to your preferences in each of these areas, what would this look like?

Response options:

$1=$ Strongly disagree

$2=$ Disagree

$3=$ Slightly disagree

$4=$ Neither agree nor disagree

$5=$ Slightly agree

$6=$ Agree

$7=$ Strongly agree

Items:

1. I prefer to work closely with other people (either 'clients', or people in related jobs in my own organization).

2. I prefer a job which permits me decision authority on my own regarding how to go about doing the work.

3. I prefer work that has an obvious beginning and end. In other words, I prefer a job in which I complete the whole process, instead of just a part of the process.

4. My preference is for a job that requires use of a variety of skills and talents.

5. I prefer work in which the results significantly affect the lives or well-being of other people.

6. I prefer frequent feedback from supervisors or coworkers to let me know how well I am doing on the job.

7. My preference is for the actual job itself to provide feedback about how well I am doing (aside from any feedback provided by supervisors or coworkers). 
JDS - Section 2P

Instructions: Listed below are a number of statements which could be used to describe a job.

Again, please indicate your preferences such that if a job were suited to your needs and wants, it would have these characteristics.

Stem for items: For my ideal work,

Response options:

$1=$ Strongly disagree

$2=$ Disagree

$3=$ Slightly disagree

$4=$ Neither agree nor disagree

$5=$ Slightly agree

$6=$ Agree

$7=$ Strongly agree

Items:

1. I prefer to use a number of complex or high-level skills.

2. I prefer cooperative work with other people.

3. I prefer to complete an entire piece of work from beginning to end.

4. I prefer to figure out how well I am doing based on the requirements and results of the job itself.

5. I prefer a simple and repetitive nature of the work, without a lot of complexity. (reverse scored)

6. I prefer a job which can be done adequately by a person working alone - without talking or checking with other people. (reverse scored)

7. I prefer frequent feedback from supervisors and coworkers about how well I am doing in my work.

8. I prefer a job in which other people are affected by how well the work gets done.

9. I prefer a job which engages my own personal initiative or judgment in carrying out the work.

10. I prefer when supervisors often let me know how well they think I am performing.

11. I prefer to completely finish the pieces of work I begin.

12. I prefer it when the results of the job itself gives obvious clues about whether or not I am performing well.

13. I prefer independence and freedom in how I do the work.

14. I prefer work that is not very significant or important in the broader scheme of things. (reverse scored) 


\section{Environment component}

\section{JDS - Section 1E}

Instructions: This part of the questionnaire asks you to describe your job, as objectively as you can.

Please do not use the part of the questionnaire to show how much you like or dislike your job. Instead, try to make your descriptions as accurate and objective as you possibly can.

Items with corresponding response options:

1. To what extent does your job require you to work closely with other people (either 'clients', or people in related jobs in your own organization)?

$1=$ Very little; dealing with other people is not at all necessary in doing the job.

$4=$ Moderately; some dealing with others is necessary.

7 = Very much; dealing with other people is an absolutely essential and crucial part of doing the job.

2. How much autonomy is there in your job? That is, to what extent does your job permit you to decide on your own how to go about doing the work?

$1=$ Very little; the job gives me almost no personal 'say' about how and when the work is done.

4 = Moderate autonomy; many things are standardized and not under my control, but I can make some decisions about the work.

$7=$ Very much; the job gives me almost complete responsibility for deciding how and when the work is done.

3. To what extent does your job involve doing a 'whole' and identifiable piece of work? In other words, is your job a complete piece of work that has an obvious beginning and end? Or is it only a small part of the overall work, which is finished by other people?

$1=$ My job is only a tiny part of the overall piece of work; the final results of my activities are not easily seen or known.

4 = My job is a moderate-sized 'chunk' of the overall piece of work; my own contribution can be seen in the final outcome.

7 = My job involves doing the whole piece of work, from start to finish; the results of my activities are easily seen in the final outcome.

4. How much variety is there in your job? That is, to what extent does the job require you to do many different things, using a variety of your skills and talents?

$1=$ Very little; the job requires me to do the same routine things over and over again.

$4=$ Moderate variety

7 = Very much; the job requires me to do many different things, using a number of different skills and talents. 
5. In general, how significant or important is your job? That is, are the results of your work likely to significantly affect the lives or well-being of other people?

1 = Not very significant; the outcomes of my work are not likely to have important effects on other people.

$4=$ Moderately significant

7 = Highly significant; the outcomes of my work can affect other people in very important ways.

6. To what extent do supervisors or coworkers let you know how well you are doing on your job?

$1=$ Very little; people almost never let me know how well I am doing.

4 = Moderately; sometimes people may give me 'feedback'; other times they may not.

7 = Very much; managers or coworkers provide me with almost constant 'feedback' about how well I am doing.

7. To what extent does doing the job itself provide you with information about your work performance? That is, does the actual work itself provide clues about how well you are doing - aside from any 'feedback' provided by supervisors or coworkers?

$1=$ Very little; the job itself is set up so I could work forever without finding out how well I'm doing.

4 = Moderately; sometimes doing the job provides 'feedback' to me; sometimes it does not.

7 = Very much; the job is set up so that I get almost constant 'feedback' as I work regarding how well I'm doing. 
JDS - Section 2E

Instructions: Listed below are a number of statements which could be used to describe a job. Indicate whether each statement is accurate or inaccurate to describe your job. Please try to be as objective as you can in deciding how accurately each statement describes your job- regardless of whether you like or dislike your job.

Stem for items: How accurate are the following statements in describing your job?

Response options:

$1=$ Very inaccurate

$2=$ Mostly inaccurate

$3=$ Slightly inaccurate

$4=$ Neither accurate nor inaccurate

$5=$ Slightly accurate

$6=$ Mostly accurate

$7=$ Very accurate

Items:

1. My job utilizes many complex or high-level skills.

2. My job provides a lot of cooperative work with other people.

3. My job is arranged so that I do not have to do an entire piece of work from beginning to end, only a part of it. (reverse scored)

4. Just doing the job provides many chances for me to figure out how well I am doing.

5. My job is basically simple and repetitive. (reverse scored)

6. My job can be done adequately by a person working alone - without having to talk to or check-in with other people. (reverse scored)

7. The supervisors and coworkers at my work almost never give 'feedback' about how well I am doing in my work. (reverse scored)

8. My job is one where a lot of other people can be affected by how well the work gets done.

9. My job does not provide opportunities to use my personal initiative or judgment in carrying out the work. (reverse scored)

10. Supervisors often let me know how well they think I am performing.

11. My job gives me opportunities to completely finish the work I begin.

12. My job itself provides very few clues about whether or not I am performing well. (reverse scored)

13. My job gives me considerable opportunity for independence and freedom in how I do the work.

14. My job itself is not very significant or important in the broader scheme of things. (reverse scored) 
Physical Symptoms Inventory - Spector \& Jex, 1998

Instructions: During the past 30 days, did you have...?

Response options:

$1=$ Not at all

$2=$ Once or twice

$3=$ Once or twice per week

$4=$ Most days

$5=$ Every day

Items:

1. An upset stomach or nausea

2. Trouble sleeping

3. Headache

4. Acid indigestion or heartburn

5. Eye strain

6. Diarrhea

7. Stomach cramps (Not menstrual)

8. Constipation

9. Ringing in the ears

10. Loss of appetite

11. Dizziness

12. Tiredness or fatigue 


\section{Professional Quality of Life Questionnaire - Stamm, 2010}

Instructions: As Emergency Responders, when you help others you have direct contact with their lives. As you may have found, your compassion for those you help can affect you in positive and negative ways. Below are some questions about your experiences, both positive and negative, as an Emergency Responder.

Consider each of the following questions about you and your current work situation. Select the number that honestly reflects how frequently you experienced these things in the last 30 days.

Response options:

$$
\begin{aligned}
& 6=\text { Never } \\
& 7=\text { Rarely } \\
& 8=\text { Sometimes } \\
& 9=\text { Often } \\
& 10=\text { Very Often }
\end{aligned}
$$

Items:

1. I am happy (reverse scored)

2. I am preoccupied with more than one person I have helped

3. I get satisfaction from being able to help people

4. I feel connected to others (reverse scored)

5. I jump or am startled by unexpected sounds

6. I feel invigorated after working with those I have helped

7. I find it difficult to separate my personal life from my life as an Emergency Responder

8. I am not as productive at work because I am losing sleep over traumatic experiences of a person I have helped

9. I think that I might have been affected by the traumatic stress of those I help

10. I feel trapped by my job as an Emergency Responder

11. Because of my work in emergency services, I have felt "on edge" about various things

12. I like my work as an Emergency Responder

13. I feel depressed because of the traumatic experiences of the people I have helped

14. I feel as though I am experiencing the trauma of someone I have helped

15. I have beliefs that sustain me (reverse scored)

16. I am pleased with how I am able to keep up with techniques and protocols to be effective at helping others

17. I am the person I always wanted to be (reverse scored)

18. My work makes me feel satisfied

19. I feel worn out because of my work as an Emergency Responder

20. I have happy thoughts and feelings about those I have helped

21. I feel overwhelmed because my work load seems endless

22. I believe I can make a difference through my work

23. I avoid certain activities or situations because they remind me of frightening experiences of the people I have helped 
24. I am proud of what I can do to help others

25. As a result of my work in emergency services, I have intrusive, frightening thoughts

26. I feel "bogged down" by the system

27. I have thoughts that I am a "success" as an Emergency Responder

28. I can't recall important parts of my work with trauma victims

29. I am a very caring person (reverse scored)

30. I am happy that I chose to do this work 


\section{Demographics}

Instructions: This section asks for demographic information. Please feel free to skip any questions you do not wish to answer.

Please indicate the following:

Items:

How long have you been worked in emergency services (years, months)?

Please indicate your current employment status:

Please indicate your branch of emergency response:

Time in current position (years, months):

Current Shift:

Time in current shift (years, months):

If your organization uses a rotating shift, please describe the timing:

Total time with current organization (years, months):

Gender:

Age:

Are you of Hispanic, Latino, or Spanish origin?

What is your ethnicity (select one or fill in)?

What is the highest level of education you have completed?

Please indicate your household's income bracket: 


\section{Appendix C}

\section{Mplus Syntax including MODEL CONSTRAINT command}

TITLE: Read data set "ERV16.dat" in MPlus

this syntax models Compassion satisfaction explained by Skill variety

DATA: file is ERV16.dat;

VARIABLE:

NAMES = ID CS BO STS PSITot

AuE DoE FaE FjE SvE TiE TsE

AuP DoP FaP FjP SvP TiP TsP

$\mathrm{CogAb}$ PJ1 PJ2 PJ3

IWM JSO KOR MOW RFW PSQOp PSQOrg

BFA BFC BFE BFN BFO TE3R;

!these are all variables in dat in file order, including auxiliary variables;

USEVARIABLES = CS SvE SvP SvE2 SvEP SvP2;

!these are the variables in use for the regression;

Missing $=$ all(-99);

DEFINE:

$\mathrm{SvE} 2=\mathrm{SvE}^{*} \mathrm{SvE}$

SvEP $=$ SvE*SvP;

$\mathrm{SvP} 2=\mathrm{SvP} * \mathrm{SvP}$

ANALYSIS:

ESTIMATOR $=\mathrm{ml}$;

BOOTSTRAP $=10000$;

MODEL:

CS on SvE SvP SvE2 SvEP SvP2 (b1 b2 b3 b4 b5);

!this is the regression and giving labels to the parameters;

SvE SvP SvE2 SvEP SvP2; !this requests IV variances;

SvE SvP SvE2 SvEP SvP2 with

SvE SvP SvE2 SvEP SvP2; !this requests IV correlations;

MODEL CONSTRAINT:

NEW(a1);

$\mathrm{a} 1=\mathrm{b} 1+\mathrm{b} 2$;

$\operatorname{NEW}(\mathrm{a} 2)$

$\mathrm{a} 2=\mathrm{b} 3+\mathrm{b} 4+\mathrm{b} 5$;

NEW(a3);

$\mathrm{a} 3=\mathrm{b} 1-\mathrm{b} 2$;

NEW(a4);

$\mathrm{a} 4=\mathrm{b} 3-\mathrm{b} 4+\mathrm{b} 5$;

OUTPUT:

standardized (stdyx) sampstat; cinterval (bootstrap); 
Permission documents for Professional Quality of Life Questionnaire - Stamm, 2010

Permission Letters

12/9/13 5:52 PM

\title{
ProQOL.org
}

\author{
HOME \\ THEORY \\ PROQOL MEASURE \& TOOLS \\ PRESENTATION AIDS \\ USE THE PROQOL \\ PROQOL DATABANK \\ RESOURCE LINKS \\ FAQ \\ ABOUT US \\ CONTACT US \\ VOLUNTEER
}

Permission Letters

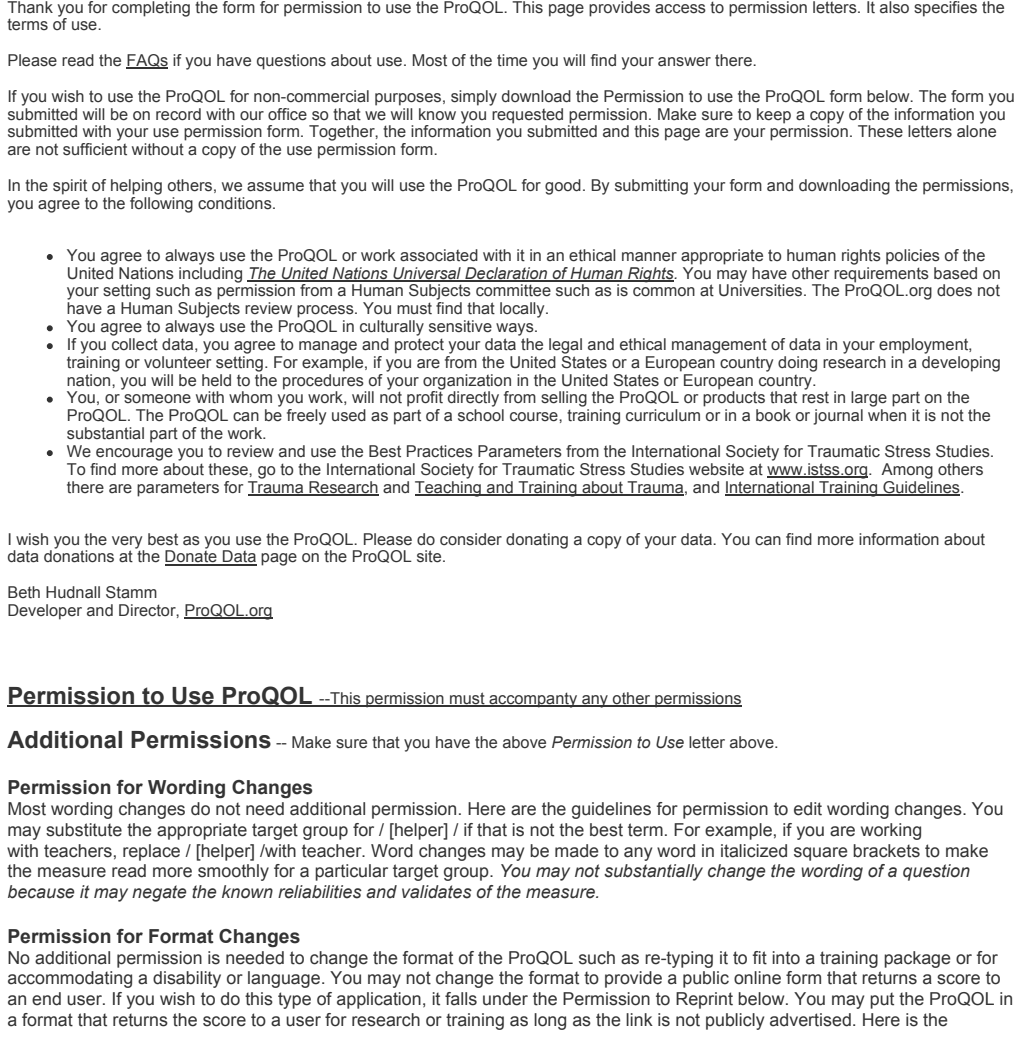

- You agree to always use the ProQOL or work associated with it in an ethical manner appropriate to human rights policies of the your setting such as permission from a Human Subjects committee such as is common at Universities. The ProQOL.org does not have a Human Subjects review process. You must find that locally.

You agree to always use the ProQOL in culturally sensitive ways.
If you collect data, you agree to manage and protect your data the legal and ethical management of data in your employment, raining or volunteer setting. For example, if you are from the United States or a European country doing research in a developing ates or European country. - You, or someone with whom you work, will not profit directly from selling the ProQOL or products that rest in large part on the
ProQOL. The ProQOL can be freely used as part of a school course, training curriculum or in a book or journal when it is not the

substantial part of the work.
We encourage you to review and use the Best Practices Parameters from the International Society for Traumatic Stress Studies. there are parameters for Trauma Research and Teaching and Training about Trauma and International Training Guidelines

I wish you the very best as you use the ProQOL. Please do consider donating a copy of your data. You can find more information about data donations at the Donate Data page on the ProQOL site.

Beth Hudnall Stamm
Developer and Director, ProQOL.org

Permission to Use ProQOL --This permission must accompanty any other permissions

Additional Permissions -- Make sure that you have the above Permission to Use letter above.

Permission for Wording Changes

Most wording changes do not need additional permission. Here are the guidelines for permission to edit wording changes. You may substitute the appropriate target group for / [helper] / if that is not the best term. For example, if you are working with teachers, replace / [helper] /with teacher. Word changes may be made to any word in italicized square brackets to make the measure read more smoothly for a particular target group. You may not substantially change the wording of a question because it may negate the known reliabilities and validates of the measure.

Permission for Format Changes

No additional permission is needed to change the format of the PrOQOL such as re-typing it to fit into a training package or for accommodating a disability or language. You may not change the format to provide a public online form that returns a score to an end user. If you wish to do this type of application, it falls under the Permission to Reprint below. You may put the ProQOL in a format that returns the score to a user for research or training as long as the link is not publicly advertised. Here is the 
guidance for format changes. You are granted permission to convert the ProQOL into other formats such as a computerized or taped version for the visually impaired. If you are required to provide documentation for changing words to make the measure more taped version for the visually impaired. If you are required to provide documentation for changing words to make
appropriate to your target population, provide the requester this page and the Permission for Use letter from above.

\section{Permission to Translate}

You will find the existing translations at measures page. They may be of use to you as your work on your translation. If you are updating one of the older versions of the ProQOL To the current, $v 5$ version, you can find the line-out comparison of the IV to 5 on the Measures

Permission to Reprint

The ProQOL is a publically available measure that is free for non-commercial use. If you wish to publish the ProQOL in a print because we work with you to make the ProQOL available to as many people as possible.

Examples of media outlets we can generally give permission for reprint without any special permission:

- $\quad$ - Print Media: newspapers, newsletters, books, journals and similar venues

data to the end user, podcast, webinars, books on tape,

news media and similar venues
- Examples of media outlets that require special negotiated permission

- Films, videos, website forms other than research program, particularly if they return automated scoring, commercial onlin

.

Of the class and other similar uses. Click here to discuss additional permissions.
- Electronic Media: non-commercial online use that do return data to the end user. Click here to discuss additional

permissions. 


\section{ProQOL.org}

\author{
HOME \\ THEORY \\ PROQOL MEASURE \& TOOLS
}

PRESENTATION AIDS

USE THE PROQOL

PROQOL DATABANK

RESOURCE LINKS

ABOUT US

CONTACT US

VOLUNTEER
Request ProQOL Use Permission

Permission to Use the ProQOL

If you would like to use the ProQOL: Compassion Satisfaction and

Compassion Fatigue for the standard permissions are granted on the measure itself (see sidebar here). We understand that there are times when a formal permissions document is helpful or necessary. If you would like a formal permission to use from us, please complete the form below.

Item Wording Changes Most wording changes are options as specified on the measure itself. The term "helper" and "helping" are generic and may not fit with your organizational or ethno- cultural community. We invite you to use terms that fit better for you. Most alteratinos are quite simple. For example, "helper" might be changed to "teacher". Sometimes changes are more complicated and there may be more issues involved than the standard word substitution. In those cases, use the contact us form.

Permissions for Translations or Editing of Existing Translations If you would like to translate the ProQOL into a language other than English we are delighted help you. We will work with you to assist with understanding the intentions and nuances of items to help improve the translations. We request that you donate a copy of the translation so that others can use it. We recognize that translations improve over time. If you would like to refine an existing translation, or help update it from the ProOQL IV, we are pleased to work with you.

Other Permissions If you would like to make changes other than those allowed under the standard use permissions or identified above, please use the contact us form and we will work with you to see how we can meet your needs and the statistical needs of the measure.
Standard Use Permissions

You may also use the ProQOL in for-profit settings such as a training course as long as the course is the item sold, not the
ProQOL which may be used in the course.

The ProQOL measure may be freely copied as long as (a) author is credited, (b) no changes are made other than those authorized below, and (c) it is not sold. You may substitute the appropriate target group for / [helper] / if that is not the best term. For example, if you are working with teachers, replace / [helper] / with teacher. Word changes may be made to any word in italicized square brackets to make the measure read more smoothly for a particular target group.

Additionally you are granted permission to convert the ProQOL into other formats such as a computerized or taped version for the visually impaired.

\begin{tabular}{|c|c|}
\hline \multicolumn{2}{|r|}{ Permissions Request } \\
\hline $\begin{array}{l}\text { YOU WILL NEED TO PRINT } \\
\text { A COPY OF THIS FORM FOR YOUR } \\
\text { FILES. MAKE SURE YOU PRINT } \\
\text { BEFORE SUBMITTING. }\end{array}$ & $\begin{array}{l}\text { To print, after you complete the form and BEFORE you submit } \\
\text { use your browser's print function. If you are using Firefox, } \\
\text { go to the drop-down menu on the top left of the } \\
\text { browser and select print. In Internet Explorer select } \\
\text { the printer icon on the tool bar. If you are still unsure, } \\
\text { check your browser's help menu. }\end{array}$ \\
\hline \multicolumn{2}{|l|}{ Starred $*$ fields are required } \\
\hline $\begin{array}{l}\text { Type of permission } \\
\text { requested. } \\
\text { Please check }\end{array}$ & $\begin{array}{l}\text { УPermission to Use } \\
\text { 『Permission for Wording Change } \\
\square \text { Permission for Format Change }\end{array}$ \\
\hline
\end{tabular}




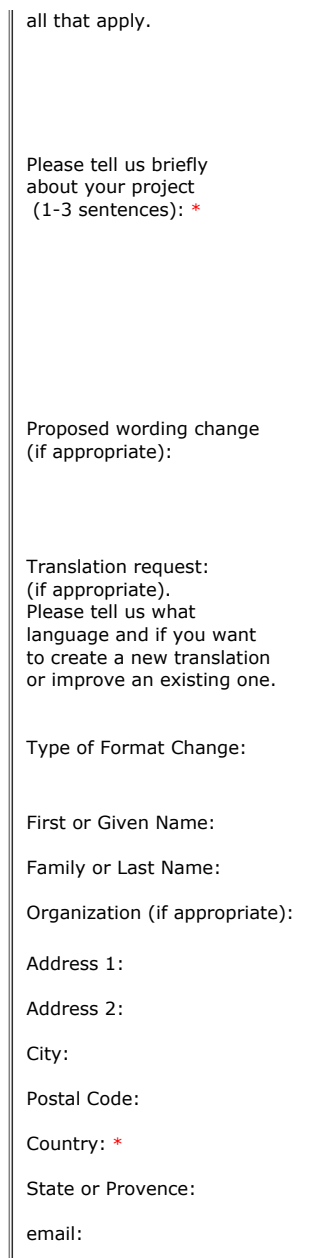

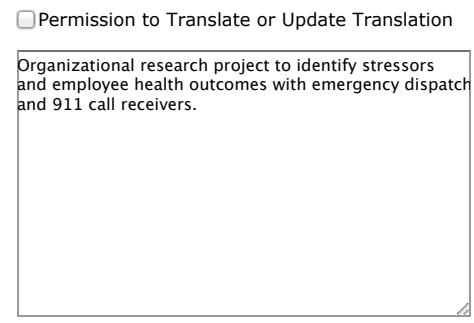

11. Because of my interactions with the people I help, I have felt "on edge" about various things.

16. I am pleased with how I am able to keep up with help.

25. As a result of my interactions with the people I help, I have intrusive, frightening thoughts.
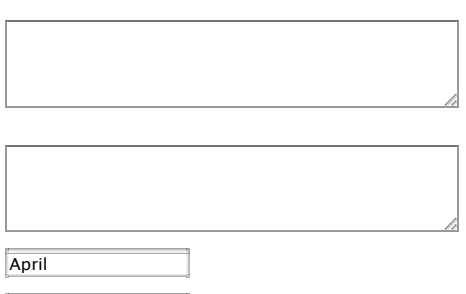

Schantz

Florida International U

11200 SW 8th Street

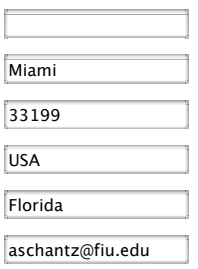

aschantz@fiu.edu

MAKE SURE TO PRINT BEFORE YOU SUBMIT

Submit Reset 
VITA

APRIL D. SCHANTZ

Born, Norfolk, Virginia

2004-2009

Human Resources Manager

Lowe's Home Centers

Construction Data Corporation

Vero Beach, Florida

2012

B.A., Psychology, with Honors, Magna Cum Laude Florida International University

Miami, Florida

2015

M.S., Psychology, with specialization in Industrial/Organizational Psychology and minor in Quantitative Methods Florida International University Miami, Florida

2016-2018

Doctoral Candidate

Florida International University

Miami, Florida

Graduate Teaching Assistant

Primary Instructor, Psychology

Florida International University

Miami, Florida

\section{PUBLICATIONS AND PRESENTATIONS}

Lanz, J. J., Falcon, A., Bruk-Lee, V., Manapragada, A., \& Schantz, A. (2016, April). Examining the nomological network of resilience. Poster presented at the 86th annual convention of the Rocky Mountain Psychological Association, Denver, CO.

Manapragada, A., Lanz, J. J., Falcon, A., Schantz, A. D., \& Bruk-Lee, V. (2014, May). Breaking the silence: An examination of employee silence behavior. Poster presented at the Annual Conference of the Society for Industrial and Organizational Psychology, Honolulu, HI.

Nixon, A.E., Lanz, J. J., Manapragada, A., Bruk-Lee, V., Schantz, A. D., \& Rodriguez, J. F. (2015). Nurse safety: How is safety climate related to affect and attitude? Work \& Stress 29(4), 401-419. doi: 10.1080/02678373.2015.1076536 
Schantz, A. D., (2013, January). Longitudinal study of transformational leadership models in the virtual team environment. Paper presented at the $14^{\text {th }}$ International Academy of Management and Business Conference, San Antonio, TX.

Schantz, A. D. (2014). A young scholar's guide to building a professional network. The Industrial-Organizational Psychologist, 51(4), 157-163. Retrieved from http://www.siop.org/tip/april14TOC.aspx

Schantz, A. D., \& Bruk-Lee, V. (2016). Workplace social stressors, drug-alcohol-tobacco use, and coping strategies. Journal of Workplace Behavioral Health, 34(4), 222-241. doi: $10.1080 / 15555240.2016 .1213638$

Schantz, A. D., \& Chang, M. (2017, April). Parental occupation effects: Academic outcomes for children of emergency responders. Poster presented at the $32^{\text {nd }}$ Annual Conference of the Society for Industrial and Organizational Psychology, Orlando, FL.

Schantz, A. D., Coxe, S., Barrantes, R., Lanz, J. \& Rauof, T. A. (2015, July). An archival data examination of team resilience. Paper session presented at the $10^{\text {th }}$ Annual Conference of Interdisciplinary Network for Group Research, Pittsburgh, PA.

Schantz, A. D., Falcon, A., Harari, M., Bruk-Lee, V., \& Viswesvaran, C. (2014). A report for the Miami-Dade Police Department, Communications Bureau: On the organizational climate and job behaviors of Police Complaint Officers (PCO) and Police Dispatchers (PD). Miami, FL: Miami-Dade Police Department.

Schantz, A. D., Falcon, A., Lanz, J., Manapragada, A., \& Bruk-Lee, V. (2015, April). Drug, alcohol, and tobacco use to cope with workplace conflict. In M. A. Clark \& L. M. Zimmerman (Chairs), Addictions, vices, and work, Oh my! Symposium conducted at the $30^{\text {th }}$ Annual Conference of the Society for Industrial and Organizational Psychology, Philadelphia, PA.

Schantz, A. D., \& Pace, V. L. (Co-Chairs). (2014, August). Leadership and teamwork in the global-virtual workplace. Symposium conducted at the $74^{\text {th }}$ Annual Meeting of the Academy of Management, Philadelphia, PA.

Schantz, A. D., \& Woods, J. M. (Co-Chairs). (2016, August). Affective convergence in teams. Symposium conducted at the $76^{\text {th }}$ Annual Meeting of the Academy of Management, Anaheim, CA.

Schantz, A. D., \& Woods, J. M. (in press). Collective fit in emergency response teams. In R. P. Gephart, C. Miller, \& K. S. Helgesson (Eds.), The Routledge companion to risk, crisis and emergency management (pp. \#-\#). London, England: Taylor \& Francis. 